\title{
Mediterranean diet adherence and cancer risk in the Netherlands
}

Citation for published version (APA):

Schulpen, M. (2020). Mediterranean diet adherence and cancer risk in the Netherlands. [Doctoral Thesis, Maastricht University]. ProefschriftMaken Maastricht. https://doi.org/10.26481/dis.20200709ms

Document status and date:

Published: 01/01/2020

DOI:

10.26481/dis.20200709ms

Document Version:

Publisher's PDF, also known as Version of record

\section{Please check the document version of this publication:}

- A submitted manuscript is the version of the article upon submission and before peer-review. There can be important differences between the submitted version and the official published version of record.

People interested in the research are advised to contact the author for the final version of the publication, or visit the DOI to the publisher's website.

- The final author version and the galley proof are versions of the publication after peer review.

- The final published version features the final layout of the paper including the volume, issue and page numbers.

Link to publication

\footnotetext{
General rights rights.

- You may freely distribute the URL identifying the publication in the public portal. please follow below link for the End User Agreement:

www.umlib.nl/taverne-license

Take down policy

If you believe that this document breaches copyright please contact us at:

repository@maastrichtuniversity.nl

providing details and we will investigate your claim.
}

Copyright and moral rights for the publications made accessible in the public portal are retained by the authors and/or other copyright owners and it is a condition of accessing publications that users recognise and abide by the legal requirements associated with these

- Users may download and print one copy of any publication from the public portal for the purpose of private study or research.

- You may not further distribute the material or use it for any profit-making activity or commercial gain

If the publication is distributed under the terms of Article $25 \mathrm{fa}$ of the Dutch Copyright Act, indicated by the "Taverne" license above, 


\title{
Mediterranean diet adherence and cancer risk in the Netherlands
}

\author{
Maya Schulpen
}


Mediterranean diet adherence and cancer risk in the Netherlands

ISBN 978-94-6380-802-6

Cover design: Maya Schulpen

Layout: Maya Schulpen

Printed by: $\quad$ ProefschriftMaken | www.proefschriftmaken.nl

(C) Copyright Maya Schulpen, Eindhoven 2020

All rights reserved. No part of this thesis may be reproduced or transmitted in any form or by any means, electronic or mechanical, including photocopying, recording, or any information storage or retrieval system, without prior permission in writing from the author, or, when appropriate, from the publishers of the publications. 


\title{
Mediterranean diet adherence and cancer risk in the Netherlands
}

\author{
PROEFSCHRIFT
}

ter verkrijging van de graad van doctor aan de Universiteit Maastricht, op gezag van de rector magnificus, prof. dr. Rianne M. Letschert, volgens het besluit van het College van Decanen, in het openbaar te verdedigen op donderdag 9 juli 2020 om 14:00 uur

door

Maya Schulpen 


\section{Promotores}

Prof. dr. ir. P.A. van den Brandt

Prof. dr. P.H.M. Peeters (Julius Centrum, UMC Utrecht)

\section{Beoordelingscommissie}

Prof. dr. T.M.C.M. de Kok (voorzitter)

Prof. dr. ir. E.J.M. Feskens (Wageningen University \& Research)

Prof. dr. A.A.M. Masclee

Prof. dr. A.M. May (Julius Centrum, UMC Utrecht)

Prof. dr. H. de Vries

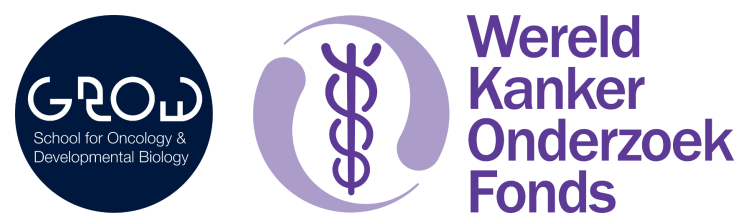

The research presented in this thesis was conducted at GROW- School for Oncology and Developmental Biology, Department of Epidemiology, of Maastricht University.

This work was financially supported by Wereld Kanker Onderzoek Fonds Nederland (WCRF$\mathrm{NL}$ ), as part of the World Cancer Research Fund International grant program (grant number 2015/1390). 


\section{Table of contents}

Chapter $1 \quad$ General introduction $\quad 7$

Chapter 2 Adherence to the Mediterranean diet and risk of lung cancer in the Netherlands Cohort Study

Chapter 3 Mediterranean diet adherence and risk of postmenopausal breast cancer: results of a cohort study and meta-analysis

Chapter $4 \quad$ Mediterranean diet adherence and risk of esophageal and gastric cancer subtypes in the Netherlands Cohort Study

Chapter 5 Mediterranean diet adherence and risk of pancreatic cancer: A pooled analysis of two Dutch cohorts

Chapter 6 Mediterranean diet adherence and risk of colorectal cancer: the prospective Netherlands Cohort Study

Chapter 7 Adherence to the Mediterranean diet and risks of prostate and bladder cancer in the Netherlands Cohort Study

Chapter 8 Adherence to the Mediterranean diet and overall cancer incidence: the Netherlands Cohort Study

Chapter 9 General discussion

Addendum Summary

Samenvatting

Valorization

Dankwoord

Curriculum Vitae 



\section{Chapter 1}

\section{General introduction}

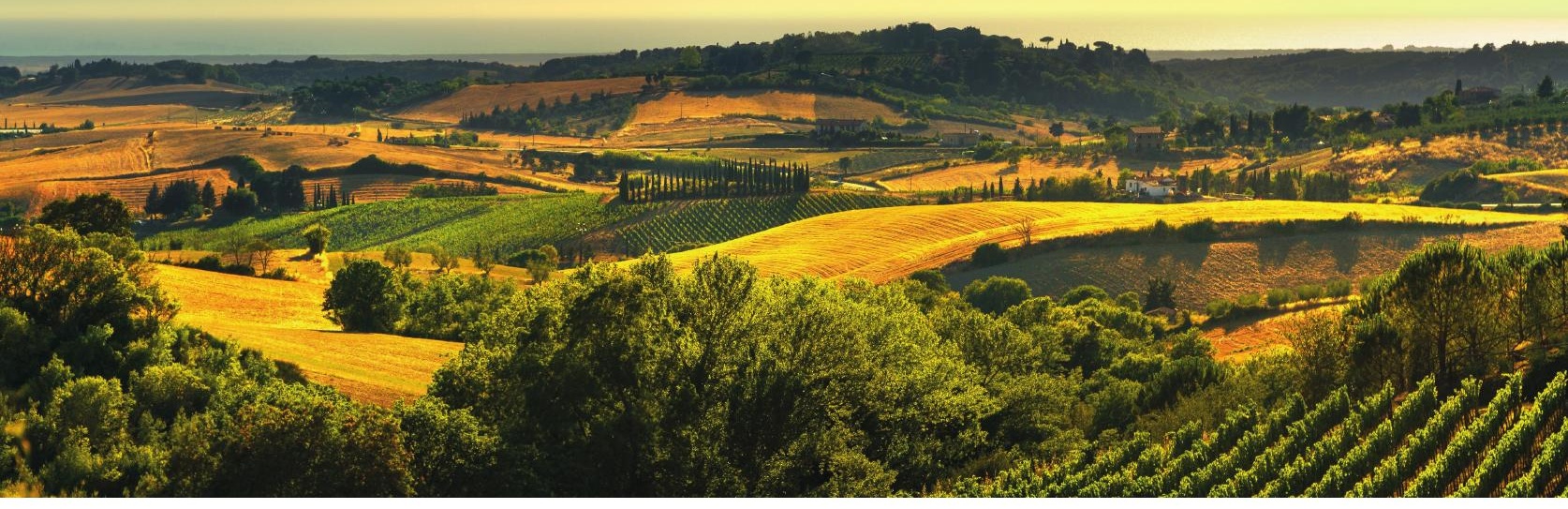


The cancer incidence in the Netherlands is high, causing a considerable social and economic burden [1-3]. After years of increase, the age-standardized incidence rate (ESR) of cancer in the Netherlands has stabilized recently to 477 per 100000 people in 2018 [4, 5]. A decreasing trend has been reported for the age-standardized mortality (ESR) of cancer [5]. Since 2008, cancer has replaced cardiovascular disease (CVD) as the leading cause of death in the Dutch population [6].

According to estimations, approximately $10 \%$ of the cancers diagnosed in the Netherlands in 2010 could be attributed to suboptimal dietary habits and were therefore preventable [7]. Recently, the traditional Mediterranean diet (MD) has gained attention as a healthy and appetizing dietary pattern. This thesis is focused on the association between MD adherence and cancer risk in the Netherlands.

\section{The Mediterranean diet}

The traditional Mediterranean diet can be defined as the dietary pattern typical of the Mediterranean regions traditionally known for olive cultivation (e.g., a large part of Greece and southern Italy) in the late 1950s and the early 1960s. In these years, areas along the Mediterranean shore had recovered from World War II, while their inhabitants had not yet adopted a more Western lifestyle characterized by fast food $[8,9]$.

Formulating a definition of the MD was a challenging task for experts in the field, since there was not one true MD. Dietary patterns varied slightly across different areas in the Mediterranean basin. To illustrate this, the Greek variant of the MD was characterized by its high total fat intake ( $\pm 40 \%$ of total energy intake), resulting from the generous consumption of olive oil. The Italian diet, on the other hand, was known for an exceptionally high consumption of pasta. Differences were also noticed between dietary patterns of other Mediterranean countries, for instance, the intake of fish was rather high in Spain [9]. However, for these areas, dietary patterns have not been described as exhaustively [8].

At the International Conference on the Diets of the Mediterranean in 1993, international experts agreed to use the typical dietary patterns of Crete, other parts of Greece, and southern Italy in the late 1950s and the early 1960s as a basis for the development of the cultural model for the MD [8], which was graphically presented by the "Mediterranean diet pyramid" (Figure 1.1). Despite inferior health care facilities, adult life expectancy was high in the abovementioned Mediterranean regions in the early 1960s, whereas diet-related chronic disease rates were low. Additionally, there was epidemiological evidence available suggesting that comparable dietary patterns were associated with several health benefits [8]. Because the diets of Crete, other parts of Greece, and southern Italy were characterized by the use of olive oil as the principal source of fat, the definition of the MD was extended to refer to the dietary patterns observed in all olive-cultivating areas bordering the Mediterranean Sea $[8,10]$. Accordingly, the MD was defined in this thesis as "the dietary pattern typical of the Mediterranean regions traditionally known for olive cultivation in the late 1950 s and the early $1960 \mathrm{~s}^{\prime \prime}$.

Despite their differences, dietary patterns found in the olive-growing areas of the Mediterranean basin generally shared the following key components: The traditional MD was mainly a plant-based diet characterized by the consumption of large amounts of fruits, vegetables, legumes, nuts and seeds, and whole grains. Total fat intake varied from moderate to high depending on the specific Mediterranean area. However, the monounsaturated to 
saturated fatty acid ratio (MUFA:SFA ratio) was high in all cases, as olive oil, which is rich in monounsaturated lipids, was the principal source of fat in the MD. Intake of milk and dairy products, poultry, and eggs was low to moderate, whereas red and processed meats, sweets, and refined grains were consumed in low quantities. Fish consumption was generally low to moderate as well, but depended largely on the distance to the sea. Finally, the MD was characterized by a moderate alcohol consumption, usually in the form of red wine during meals. In general, foods consumed in the Mediterranean region were minimally processed, fresh, seasonal, organic, and cultivated by local farmers $[8,9]$.

\section{Mediterranean Diet Pyramid}

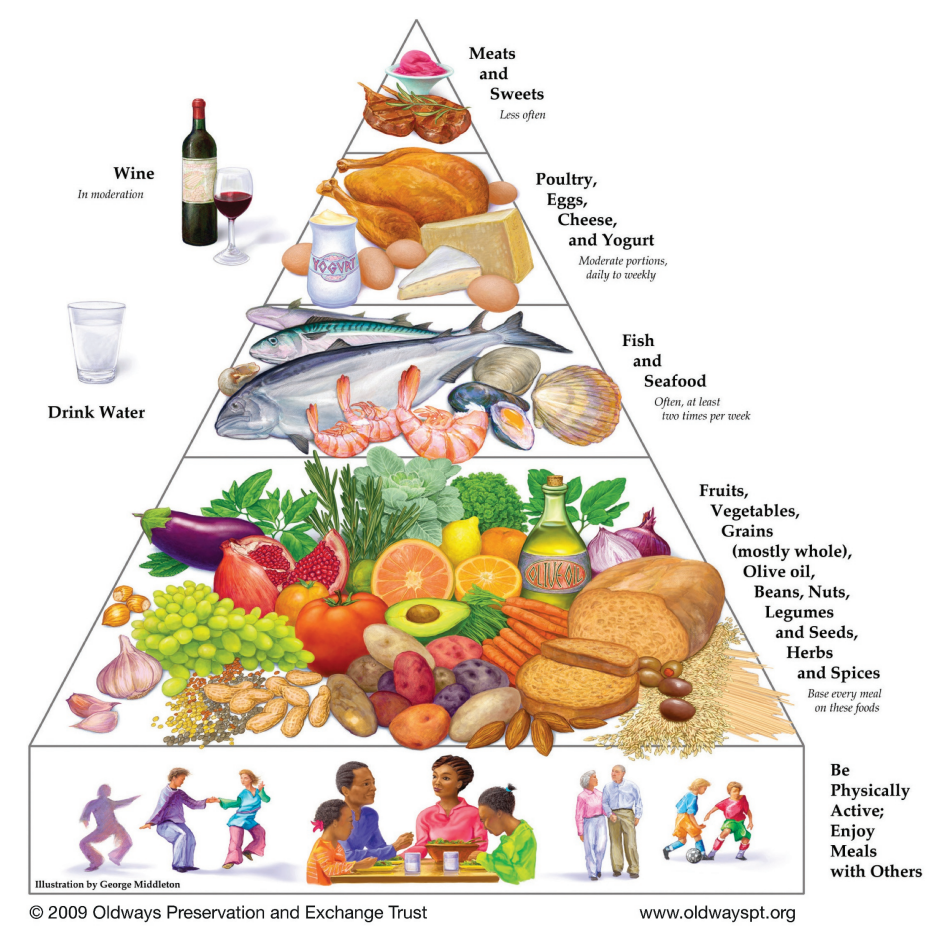

Figure 1.1 Mediterranean diet pyramid (https://oldwayspt.org)

\section{Health benefits of the Mediterranean diet}

Despite the inferior health care facilities in Mediterranean regions, Mediterranean populations between 1960 and 1990 experienced lower mortality rates and a higher adult life expectancy than populations of northern Europe and North-America [9]. One of the candidate factors proposed to explain this remarkable finding was the plant-based dietary pattern to which Mediterranean populations adhered [9].

Groundbreaking in the discovery of the beneficial health effects of the MD was the Seven Countries Study, initiated mid-twentieth century by Ancel Keys. This study followed almost 13000 men between the ages of 40 and 59 years, who were enrolled in 16 centers, distributed over seven countries, for up to 30 years. The main goal was to investigate the relationship between diet and the incidence of coronary heart disease (CHD). Based on the 
results, Keys concluded that the lower rates of CHD observed in Mediterranean populations could be attributed to low dietary levels of saturated fat $[9,11]$.

Since the Seven Countries Study, the association between adherence to the MD and numerous health outcomes has been investigated extensively in observational studies and a number of randomized controlled trials (RCTs). The current scientific evidence suggests that higher MD adherence might be associated with reduced rates of overall mortality, CVD incidence and mortality, overall cancer incidence, neurodegenerative diseases, and diabetes [12-14]. However, the evidence for other health outcomes, e.g., many specific cancer types, metabolic risk factors, and inflammatory parameters, is weaker or controversial [12-14]. So far, several RCTs have been conducted on this topic, primarily investigating the effect of the MD pattern on CVD risk (factors) [14]. Particularly noteworthy are the Lyon Diet Heart Study (secondary prevention trial among survivors of a first acute myocardial infarction, [15, 16]) and the Prevención con Dieta Mediterránea trial (PREDIMED, primary prevention trial among participants who were at high risk for CVD, [17]). In these RCTs, MD interventions enriched with $\alpha$-linolenic acid (Lyon Diet Heart Study) or supplemented with either extravirgin olive oil or nuts (PREDIMED) were compared to a prudent Western-type diet (Lyon Diet Heart Study) and a low-fat control diet (PREDIMED), respectively. In addition to reduced risks of composite outcomes of major cardiovascular events [16, 18], participants in the MD intervention groups seemed to have (non-)significantly lowered risks of overall cancer (Lyon Diet Heart Study) and postmenopausal breast cancer (PREDIMED) [19, 20].

Though results of the Lyon Diet Heart Study and PREDIMED trial indicated that adherence to a MD might lower cancer risk, the analyses were based on small numbers of incident cancer cases. The large majority of the evidence for the potential relation between MD adherence and cancer risk has been gathered from observational studies. MD adherence (highest vs. lowest quantile) was associated with a statistically significantly reduced overall cancer incidence (4\%) and mortality (14\%) in cohort studies [21]. Additionally, a recent metaanalysis of observational studies by Schwingshackl and colleagues [22] showed statistically significant inverse associations between MD adherence and incidence of cancers of the colorectum, breast (postmenopausal), prostate, stomach, liver, head and neck, gallbladder, and biliary tract. However, the evidence was limited for most of the investigated cancer sites, individual studies sometimes had inconsistent results, and many of the included studies had case-control designs. Due to the retrospective nature of the case-control design and the associated vulnerability of this design to several types of bias (e.g., recall and selection biases), the prospective cohort design is preferred. Different etiologies have been suggested for subtypes of several cancer sites, such as the lung [23], breast [24], esophagus [25], stomach [26], and colorectum [27, 28]. Nevertheless, prospective studies rarely evaluated associations with MD adherence separately for subtypes of cancer sites, which could have contributed to the inconsistent findings. Furthermore, potential heterogeneity of associations across the sexes requires more attention. Accordingly, additional research evaluating the association of MD adherence with cancer risk is desired. Ideally, future studies would have a prospective design and would differentiate between men and women as well as cancer subtypes.

The potentially beneficial influence of MD adherence on cancer risk seems biologically plausible. Nevertheless, the specific pathways involved still remain to be elucidated. Two of the more established mechanisms of action concern the antioxidant and anti-inflammatory properties of the MD, which could counteract oxidative damage to DNA, proteins, and lipids, and reduce inflammation, respectively $[29,30]$. The plant-based nature of the MD ensures 
a high intake of antioxidants (e.g., vitamins, minerals, and polyphenols) derived from the consumption of vegetables, fruits, nuts, whole grains, olive oil, and other plant foods [29-32]. Correspondingly, elevated total antioxidant capacity levels have been observed in subjects with higher MD adherence [33]. Furthermore, adherence to the MD has generally been associated with reduced levels of markers of low-grade inflammation (e.g., interleukin-6 and C-reactive protein) [34]. The ability of the MD to reduce inflammation might (partly) be attributed to the abundance of polyphenols and omega- 3 polyunsaturated fatty acids (PUFA) in this dietary pattern [29-31, 35]. In addition to its antioxidant and antiinflammatory effects, MD adherence might reduce the biological activity of hormones and growth factors that have been implicated in the stimulation of tumor development and growth, such as insulin, estrogens, androgens, and insulin-like growth factor 1 [29]. Other proposed mechanisms that might mediate the potential cancer-protective effect of the MD are among others related to the high intake of dietary fiber, the low intake of red and processed meats, body weight regulation, decreased activation of nutrient sensing pathways [e.g., the pro-carcinogenic mechanistic target of rapamycin (mTOR) pathway] through amino acid restriction, and modulation of the composition and function of the gut microbiota influencing their metabolite secretion [29, 30, 32, 36-38].

\section{Methods to assess Mediterranean diet adherence}

To investigate the association of the MD with cancer risk, researchers usually assess the level of adherence to the MD of study participants: In other words, the extent to which a subject's diet resembled the MD pattern.

The level of MD adherence in a certain population can be assessed using either a priori or a posteriori scores $[39,40]$. In case of the MD, the a priori approach seems to have been applied most frequently. In this approach, food groups characteristic of the MD, because of their relatively high or low consumption in this dietary pattern, are selected based on scientific knowledge and evidence, and are combined into indexes assessing the overall level of MD adherence. Subsequently, an individual's level of MD adherence is derived from his/her dietary information using the created index and associations between MD adherence and health outcomes are investigated. Generally, a priori defined indexes assess MD adherence in a rather qualitative way [40].

As an alternative to the usage of a priori defined indexes, the health effects of the MD have occasionally been investigated in studies that observed a MD-like dietary pattern in their study population after applying a posteriori methods. Using the a posteriori approach, e.g., factor or cluster analysis is carried out to empirically derive dietary patterns from the collected dietary data. In factor analysis, correlations between food groups are used to extract food patterns with the goal to explain as much of the variance in the dietary data as possible. Subsequently, a summary score is calculated for each of the extracted food patterns. Furthermore, dietary patterns in the study population can be obtained by grouping individuals based on their food intakes into mutually exclusive clusters, as is done in cluster analysis [39, 41, 42]. The a posteriori approach has some limitations, the major one being its subjectivity. It is up to the researchers to decide upon, e.g., the categorization of the dietary data into food groups, the number of dietary patterns observed in the study population, and the interpretation and naming of the derived patterns. Additionally, a posteriori defined dietary patterns are specific for the study population, making comparison with dietary patterns observed in other studies challenging. Finally, the observed dietary pattern does not necessarily reflect an "ideal" MD [39, 41-43]. 
From this point onwards, this thesis will focus on a priori defined indexes used in the assessment of MD adherence.

\subsection{The Mediterranean diet score by Trichopoulou et al.}

The $a$ priori defined traditional Mediterranean diet score (tMED) created by Trichopoulou et al. $[44,45]$ is a straightforward and convenient tool to measure the level of MD adherence. tMED [44] assesses the relative level of MD adherence based on the mean daily intakes of eight dietary components, which are characteristically consumed in relatively high or low amounts in the traditional Greek MD. Each dietary component receives a score of 0 or 1 point using sex-specific median values in the study population as cut-offs (except for alcohol intake). Subsequently, the scores of the individual components are added to create a sum score, with the maximum value of 8 points reflecting closest adherence to the MD. A score of 1 point is assigned to mean daily intakes at or above the sex-specific median of presumed beneficial dietary components, which include vegetables (excluding potatoes), fruits and nuts, legumes, and cereals (including potatoes). Inverse scoring is applied to the meat and dairy components, which are presumed to have detrimental health effects. Finally, 1 point is assigned to a moderate alcohol intake (men: $10-50 \mathrm{~g} /$ day, women: 5-25 g/day) and a high ( $\geq$ sex-specific median) MUFA:SFA ratio [44]. Before calculation of tMED, dietary intakes are standardized to energy intakes of $2500 \mathrm{kcal}$ and $2000 \mathrm{kcal}$ per day for men and women, respectively, to correct for differences in daily energy intake $[44,46]$.

tMED is the most widely used index in the assessment of MD adherence and numerous variants have appeared since its introduction in 1995 [40]. In this paragraph, we will discuss the most important tMED variants, which are summarized in Table 1.1. The dietary components composing the original tMED are displayed in bold in this table. Scores are displayed for intakes at or above the sex-specific median, unless otherwise specified.

Firstly, a revised version of tMED was proposed by Trichopoulou et al. in 2003, which included fish as an additional beneficial component, whereas potato intake was removed from the cereal group [45]. In 2005, two frequently used variations of the revised tMED were independently introduced, namely the modified Mediterranean diet score (mMED, [47]) and the alternate Mediterranean diet score (aMED, [46, 48]). In MMED, the ratio of unsaturated fatty acids (MUFA + PUFA) to SFA replaces the MUFA:SFA ratio to increase the usability of the score in non-Mediterranean populations [47]. Dietary patterns consistently proven to decrease chronic disease risk formed the foundation for the formulation of aMED, which was particularly developed for use in the US $[46,48]$. In aMED, fruits and nuts are included as two separate components, only whole grains are considered in the cereal group, the meat component is restricted to red and processed meats, and dairy products are excluded from the score. Furthermore, moderate alcohol consumption is defined as $5-25 \mathrm{~g} /$ day for both men and women [46, 48]. Revised tMED, mMED, and aMED range from 0 to 9 points. A final and less commonly applied variant of revised tMED is the relative Mediterranean diet score (rMED), introduced by Buckland et al. in 2009 [49]. Different from the other scores, rMED uses a tertile-based scoring for all components (except alcohol) and accordingly has a maximum value of 18 points. Additionally, rMED directly scores olive oil consumption instead of using the MUFA:SFA ratio as a substitute [49]. 


\section{Chapter 1}

Table 1.1 The five major variants of the Mediterranean diet score initially developed by Trichopoulou et al. in 1995 (scores are displayed for intakes $\geq$ sex-specific median, unless otherwise specified)

\begin{tabular}{|c|c|c|c|c|c|}
\hline Dietary component & $\begin{array}{l}\text { tMED } \\
\text { (1995) }\end{array}$ & $\begin{array}{l}\text { Revised tMED } \\
\text { (2003) }\end{array}$ & $\begin{array}{l}\text { mMED }^{a} \\
(2005)\end{array}$ & $\begin{array}{l}\text { aMED }^{a} \\
(2005)\end{array}$ & $\begin{array}{l}\text { rMED }^{b} \\
\text { (2009) }\end{array}$ \\
\hline Vegetables (excl. potatoes) & 1 & 1 & 1 & 1 & 2 \\
\hline Fruits and nuts & 1 & 1 & 1 & & 2 \\
\hline Fruits (incl. juices) & & & & 1 & \\
\hline Nuts & & & & 1 & \\
\hline Legumes & 1 & 1 & 1 & 1 & 2 \\
\hline Cereals (incl. potatoes) & 1 & & & & \\
\hline Cereals (excl. potatoes) & & 1 & 1 & & 2 \\
\hline Whole grains & & & & 1 & \\
\hline Fish and seafood & & 1 & 1 & 1 & 2 \\
\hline Meat and meat products & 0 & 0 & 0 & & 0 \\
\hline Red and processed meat & & & & 0 & \\
\hline Dairy products & 0 & 0 & 0 & & 0 \\
\hline MUFA:SFA ratio & 1 & 1 & & 1 & \\
\hline (MUFA + PUFA):SFA ratio & & & 1 & & \\
\hline Olive oil & & & & & 2 \\
\hline
\end{tabular}

\section{Moderate alcohol intake}

Men: $10-50$ g/day

Women: 5-25 g/day
1 0-8

1

1

$\begin{array}{lll}1 & 1: 5-25 \text { g/day } & 2^{c} \\ 1 & 1: 5-25 \text { g/day } & 2^{\text {c }}\end{array}$

Range score 0-9 0-9 0-9

Abbreviations: tMED, traditional Mediterranean diet score; mMED, modified Mediterranean diet score; aMED, alternate Mediterranean diet score; $\mathrm{rMED}$, relative Mediterranean diet score; MUFA, monounsaturated fatty acids; SFA, saturated fatty acids; PUFA, polyunsaturated fatty acids

a Scores of 0 or 1 point were assigned to each dietary component using sex-specific median values as cut-off, unless otherwise specified. Scores are displayed for intakes $\geq$ sex-specific median.

${ }^{\mathrm{b}}$ Scores ranging from 0 to 2 points were assigned based on tertiles of intakes, unless otherwise specified. Scores for the highest tertiles are displayed.

${ }^{c} \mathrm{~A}$ score of 0 was assigned to alcohol consumption levels outside this range. 
Overall, evidence on the validity and reliability of tMED (variants) and other MD scores is scarce [50]. High intakes of typically Mediterranean foods (e.g., vegetables, legumes, fruits and nuts, and olive oil) were, as expected, increasingly common among Greek subjects with greater MD adherence according to revised tMED, whereas opposite trends were observed for intakes of meat and dairy products [45]. Additionally, the ability of the food frequency questionnaire (FFQ) to rank subjects according to their level of MD adherence has been studied by comparing MD scores (tMED variants) derived from FFQ data to MD scores based on ten or more 24-hour dietary recalls, and was judged to be reasonable [51]. Finally, the validity of tMED and variants has been confirmed by examining their competence to predict various health outcomes. For instance, higher MD adherence, measured by tMED (variants), has been associated with reduced all-cause mortality and, more specifically, reduced risks of and mortality from CVD and cancer, in a variety of Mediterranean and non-Mediterranean populations (e.g., [44-47, 49, 52-56]). To the best of our knowledge, no studies have been published yet evaluating the reliability, in the sense of reproducibility, of tMED or variants [50].

A potential limitation of tMED (variants) is the use of population medians as cut-offs to distinguish between subjects with high and low intakes of (non-)Mediterranean foods. Although subjects with high MD scores adhere more closely to the MD than subjects with lower scores, their dietary intakes do not necessarily comply with a traditional MD. This is particularly relevant in non-Mediterranean study populations. Furthermore, the use of population-specific cut-offs might hamper between-study comparisons and generalizability of study results. Another potential weakness is the assignment of equal weights to all dietary components. Finally, tMED and the majority of its variants categorize subjects as having either high or low intakes of the individual dietary components. This binary approach may result in a loss of information and reduce the discriminative capacity of the score.

In this thesis, we used aMED and mMED to determine the level of MD adherence, as will be discussed more comprehensively in the paragraph concerning the study design.

\section{Rationale and aims of the thesis}

Despite the increasing interest in the potentially favorable effect of MD adherence on cancer risk, the current evidence is still insufficient, sometimes inconsistent, and predominantly based on case-control studies. Additional prospective studies are desired, which might enable the formulation of more definitive conclusions.

The general aim of this thesis was to prospectively investigate the association between MD adherence and cancer incidence in the Netherlands. Analyses were conducted considering the incidence of overall cancer, and cancers of the lung, breast, esophagus, stomach, pancreas, colorectum, prostate, and bladder as primary outcomes. The specific cancer types of interest were selected because of their high incidence, expected relation with the MD, or poor prognosis. In view of the potential role of the MD in cancer prevention, it is also interesting to evaluate the relation of this dietary pattern with overall cancer incidence. We specifically addressed subtypes of the selected cancer sites and estimated associations separately for men and women, whenever possible. Furthermore, model performances of two MD scores (aMED and mMED) were compared and the effect of exclusion of alcohol from the MD scores was evaluated. As a final aim of this thesis, we compared the populationdependent MD scores to absolute scores based on the dietary recommendations for cancer 
prevention issued by the World Cancer Research Fund/American Institute for Cancer Research (WCRF/AICR) in 2007 [57].

\section{Study design}

The aims of this thesis were primarily investigated within the context of the large prospective Netherlands Cohort Study on Diet and Cancer (NLCS). The association of MD adherence with pancreatic cancer risk was evaluated by pooling results from the NLCS and the Dutch cohort of the European Prospective Investigation into Cancer and Nutrition (EPIC-NL) to increase the statistical power. Detailed descriptions of the population-based NLCS and EPICNL cohorts have been published previously [58-64].

The study population of the NLCS consists of 58279 men and 62573 women, who originated from 204 Dutch municipalities and were aged 55 to 69 years at the initiation of the study in September 1986 [58]. NLCS participants filled out a self-administered baseline questionnaire on cancer risk factors, which included a FFQ $[60,65]$. To allow for efficient data processing and analysis, the NLCS used the nested case-cohort design [58, 61, 66]. Accordingly, incident cancer cases were detected in the entire NLCS cohort, while accumulated person-years at risk in the cohort were estimated based on a random subcohort $(N=5000)$ that was sampled immediately after baseline. Vital status of subcohort members was checked biennially using municipal population registries.

The EPIC-NL cohort is composed of the EPIC-Prospect and EPIC-MORGEN cohorts, which collectively enrolled 40011 subjects [62-64]. EPIC-Prospect includes 17357 women, aged 49 to 70 years, who participated in a breast cancer screening program in the region of Utrecht. EPIC-MORGEN encompasses 10260 men and 12394 women, aged 20 to 65 years, who were randomly sampled from the general population of three Dutch towns (Amsterdam, Maastricht, and Doetinchem). Between 1993 and 1997, baseline data were obtained using a general questionnaire, a FFQ, and a physical examination. EPIC-NL participants were followed up for vital status using municipal population registries.

In both cohorts, incident cancer diagnoses were identified annually by record linkage with the Netherlands Cancer Registry and the nationwide Dutch Pathology Registry (PALGA) [59, 64]. NLCS participants were followed up for 20.3 years until December 31, 2006. The median follow-up in EPIC-NL was 19.2 years (baseline to December 31, 2014).

Information concerning the dietary habits of NLCS and EPIC-NL participants during the year preceding enrolment was acquired using semi-quantitative FFQs. These FFQs were study-specific, and their validity and reproducibility have been evaluated $[60,65,67,68]$. Additionally, Dutch food composition (NEVO) tables were utilized to derive mean daily nutrient intakes from the FFQ data [69].

This thesis is mainly focused on the MD pattern as a whole, rather than its individual nutritional components. The pattern-based approach allowed us to investigate the combined effect of all aspects of the MD taking potential interactions between single dietary factors or food groups into account. In order to assess the relative level of MD adherence, we used aMED $[46,48]$ and mMED [47], which are both variants of tMED [44, 45]. As described above, tMED and variants are straightforward and user-friendly tools to assess MD adherence. Additionally, the wide usage of these MD scores facilitates the comparability of our study results to previously published findings on the topic. The decision to use aMED instead of revised tMED was based on the consistently better model fit of aMED in earlier NLCS mortality analyses [54]. Additionally, we included mMED, which was specifically adapted to 
measure MD adherence in non-Mediterranean populations. Because alcohol may increase risks of multiple types of cancer even at low or moderate levels of consumption [36, 70], we created reduced variants of aMED and MMED (aMEDr and mMEDr, respectively) that did not contain the alcohol component.

\section{Outline of the thesis}

The following chapters of this thesis (Chapter 2-7) focus on associations of MD adherence with risks of specific cancer sites. We investigated relations of MD adherence with risks of cancers of the lung (Chapter 2), breast (Chapter 3), esophagus and stomach (Chapter 4), pancreas (Chapter 5), colorectum (Chapter 6), and prostate and bladder (Chapter 7). Associations with MD adherence may differ across (subtypes of) cancer sites. Therefore, from the perspective of cancer prevention, it would be helpful to get an impression of the association between MD adherence and overall cancer risk, which is the topic of Chapter 8. Chapter 9 concludes this thesis with a reflection on the main findings and some methodological considerations. Furthermore, implications for public health and recommendations for future research are discussed in this final chapter. 


\section{References}

1. Bray F, Ferlay J, Soerjomataram I, Siegel RL, Torre LA, Jemal A. Global cancer statistics 2018: GLOBOCAN estimates of incidence and mortality worldwide for 36 cancers in 185 countries. CA Cancer J Clin. 2018;68(6):394-424.

2. Ferlay J, Ervik M, Lam F, Colombet M, Mery L, Pineros M, et al. Global Cancer Observatory: Cancer Today. Lyon, France: International Agency for Research on Cancer; 2018 [cited 2019 November]. Available from: https://gco.iarc.fr/today.

3. Ferlay J, Colombet M, Soerjomataram I, Mathers C, Parkin DM, Pineros M, et al. Estimating the global cancer incidence and mortality in 2018: GLOBOCAN sources and methods. Int J Cancer. 2019;144(8):1941-53.

4. Siesling S, Visser O, Aarts MJ, Verhoeven RHA, Aben KKH, Dinmohamed AG, et al. [Fight against cancer in the Netherlands: current state of affairs]. Ned Tijdschr Geneeskd. 2019;163 (in Dutch).

5. Nederlandse Kankerregistratie, operated by IKNL: IKNL [cited 2019 October]. Available from: http://www.cijfersoverkanker.nl/.

6. StatLine - Gezondheid, leefstijl, zorggebruik en -aanbod, doodsoorzaken; vanaf 1900 [Internet]. Statistics Netherlands. 2019 [cited 2019 October]. Available from: https://opendata.cbs.nl/ statline/\#/CBS/nl/dataset/37852/table?ts=1570021132658 (in Dutch).

7. Lanting $\mathrm{Cl}$, de Vroome EM, Elias SG, van den Brandt PA, van Leeuwen FE, Kampman E, et al. [Contribution of lifestyle factors to cancer: secondary analysis of Dutch data over 2010 and a projection for 2020]. Ned Tijdschr Geneeskd. 2014;159:A8085 (in Dutch).

8. Willett WC, Sacks F, Trichopoulou A, Drescher G, Ferro-Luzzi A, Helsing E, et al. Mediterranean diet pyramid: a cultural model for healthy eating. Am J Clin Nutr. 1995;61(6 Suppl):1402S-6S.

9. Trichopoulou A, Lagiou P. Healthy traditional Mediterranean diet: an expression of culture, history, and lifestyle. Nutr Rev. 1997;55(11 Pt 1):383-9.

10. Nestle M. Mediterranean diets: historical and research overview. Am J Clin Nutr. 1995;61(6 Suppl):1313S-20S.

11. Kromhout D, Menotti A, Blackburn H, (eds). The Seven Countries Study: A scientific adventure in cardiovascular disease epidemiology. Utrecht, the Netherlands: Brouwer Offset b.v.; 1994. 219 p.

12. Dinu M, Pagliai G, Casini A, Sofi F. Mediterranean diet and multiple health outcomes: an umbrella review of meta-analyses of observational studies and randomised trials. Eur J Clin Nutr. 2018;72(1):30-43.

13. Martinez-Lacoba R, Pardo-Garcia I, Amo-Saus E, Escribano-Sotos F. Mediterranean diet and health outcomes: a systematic meta-review. Eur J Public Health. 2018;28(5):955-61.

14. Serra-Majem L, Roman-Vinas B, Sanchez-Villegas A, Guasch-Ferre M, Corella D, La Vecchia C. Benefits of the Mediterranean diet: Epidemiological and molecular aspects. Mol Aspects Med. 2019;67:1-55.

15. de Lorgeril M, Renaud S, Mamelle N, Salen P, Martin JL, Monjaud I, et al. Mediterranean alpha-linolenic acid-rich diet in secondary prevention of coronary heart disease. Lancet. 1994;343(8911):1454-9.

16. de Lorgeril M, Salen P, Martin JL, Monjaud I, Delaye J, Mamelle N. Mediterranean diet, traditional risk factors, and the rate of cardiovascular complications after myocardial infarction: final report of the Lyon Diet Heart Study. Circulation. 1999;99(6):779-85.

17. Martinez-Gonzalez MA, Corella D, Salas-Salvado J, Ros E, Covas MI, Fiol M, et al. Cohort profile: design and methods of the PREDIMED study. Int J Epidemiol. 2012;41(2):377-85.

18. Estruch R, Ros E, Salas-Salvado J, Covas MI, Corella D, Aros F, et al. Primary Prevention of Cardiovascular Disease with a Mediterranean Diet Supplemented with Extra-Virgin Olive Oil or Nuts. N Engl J Med. 2018;378(25):e34.

19. de Lorgeril M, Salen P, Martin JL, Monjaud I, Boucher P, Mamelle N. Mediterranean dietary pattern in a randomized trial: prolonged survival and possible reduced cancer rate. Arch Intern Med. 1998;158(11):1181-7.

20. Toledo E, Salas-Salvado J, Donat-Vargas C, Buil-Cosiales P, Estruch R, Ros E, et al. Mediterranean Diet and Invasive Breast Cancer Risk Among Women at High Cardiovascular Risk in the PREDIMED Trial: A Randomized Clinical Trial. JAMA Intern Med. 2015;175(11):1752-60. 
21. Bloomfield HE, Koeller E, Greer N, MacDonald R, Kane R, Wilt TJ. Effects on Health Outcomes of a Mediterranean Diet With No Restriction on Fat Intake: A Systematic Review and Meta-analysis. Ann Intern Med. 2016;165(7):491-500.

22. Schwingshackl L, Schwedhelm C, Galbete C, Hoffmann G. Adherence to Mediterranean Diet and Risk of Cancer: An Updated Systematic Review and Meta-Analysis. Nutrients. 2017;9(10).

23. de Groot P, Munden RF. Lung cancer epidemiology, risk factors, and prevention. Radiol Clin North Am. 2012;50(5):863-76.

24. Chen WY, Colditz GA. Risk factors and hormone-receptor status: epidemiology, risk-prediction models and treatment implications for breast cancer. Nat Clin Pract Oncol. 2007;4(7):415-23.

25. Kamangar F, Chow WH, Abnet CC, Dawsey SM. Environmental causes of esophageal cancer. Gastroenterol Clin North Am. 2009;38(1):27-57, vii.

26. Crew KD, Neugut Al. Epidemiology of gastric cancer. World J Gastroenterol. 2006;12(3):354-

62.

27. Lee GH, Malietzis G, Askari A, Bernardo D, Al-Hassi HO, Clark SK. Is right-sided colon cancer different to left-sided colorectal cancer? - a systematic review. Eur J Surg Oncol. 2015;41(3):300-8.

28. Paschke S, Jafarov S, Staib L, Kreuser ED, Maulbecker-Armstrong C, Roitman M, et al. Are Colon and Rectal Cancer Two Different Tumor Entities? A Proposal to Abandon the Term Colorectal Cancer. Int J Mol Sci. 2018;19(9).

29. Tosti V, Bertozzi B, Fontana L. Health Benefits of the Mediterranean Diet: Metabolic and Molecular Mechanisms. J Gerontol A Biol Sci Med Sci. 2018;73(3):318-26.

30. Schwingshackl L, Morze J, Hoffmann G. Mediterranean diet and health status: Active ingredients and pharmacological mechanisms. Br J Pharmacol. 2019.

31. Brill JB. The Mediterranean diet and your health. Am J Lifestyle Med. 2009;3(1):44-56.

32. Verberne L, Bach-Faig A, Buckland G, Serra-Majem L. Association between the Mediterranean diet and cancer risk: a review of observational studies. Nutr Cancer. 2010;62(7):860-70.

33. Pitsavos C, Panagiotakos DB, Tzima N, Chrysohoou C, Economou M, Zampelas A, et al. Adherence to the Mediterranean diet is associated with total antioxidant capacity in healthy adults: the ATTICA study. Am J Clin Nutr. 2005;82(3):694-9.

34. Calder PC, Ahluwalia N, Brouns F, Buetler T, Clement K, Cunningham K, et al. Dietary factors and low-grade inflammation in relation to overweight and obesity. Br J Nutr. 2011;106 Suppl 3:S5-78.

35. Rahman I, Biswas SK, Kirkham PA. Regulation of inflammation and redox signaling by dietary polyphenols. Biochem Pharmacol. 2006;72(11):1439-52.

36. World Cancer Research Fund / American Institute for Cancer Research. Diet, nutrition, physical activity and cancer: a global perspective. Continuous update project expert report 2018. 2018. Available from: https://www.wcrf.org/dietandcancer.

37. Romaguera D, Norat T, Vergnaud AC, Mouw T, May AM, Agudo A, et al. Mediterranean dietary patterns and prospective weight change in participants of the EPIC-PANACEA project. Am J Clin Nutr. 2010;92(4):912-21.

38. Moller ME, Dahl R, Bockman OC. A possible role of the dietary fibre product, wheat bran, as a nitrite scavenger. Food Chem Toxicol. 1988;26(10):841-5.

39. Hu FB. Dietary pattern analysis: a new direction in nutritional epidemiology. Curr Opin Lipidol. 2002;13(1):3-9.

40. Bach A, Serra-Majem L, Carrasco JL, Roman B, Ngo J, Bertomeu I, et al. The use of indexes evaluating the adherence to the Mediterranean diet in epidemiological studies: a review. Public Health Nutr. 2006;9(1A):132-46.

41. Newby PK, Tucker KL. Empirically derived eating patterns using factor or cluster analysis: a review. Nutr Rev. 2004;62(5):177-203.

42. Moeller SM, Reedy J, Millen AE, Dixon LB, Newby PK, Tucker KL, et al. Dietary patterns: challenges and opportunities in dietary patterns research an Experimental Biology workshop, April 1, 2006. J Am Diet Assoc. 2007;107(7):1233-9.

43. Jacques PF, Tucker KL. Are dietary patterns useful for understanding the role of diet in chronic disease? Am J Clin Nutr. 2001;73(1):1-2.

44. Trichopoulou A, Kouris-Blazos A, Wahlqvist ML, Gnardellis C, Lagiou P, Polychronopoulos E, et al. Diet and overall survival in elderly people. BMJ. 1995;311(7018):1457-60.

45. Trichopoulou A, Costacou T, Bamia C, Trichopoulos D. Adherence to a Mediterranean diet 


\section{Chapter 1}

and survival in a Greek population. N Engl J Med. 2003;348(26):2599-608.

46. Mitrou PN, Kipnis V, Thiebaut AC, Reedy J, Subar AF, Wirfalt E, et al. Mediterranean dietary pattern and prediction of all-cause mortality in a US population: results from the NIH-AARP Diet and Health Study. Arch Intern Med. 2007;167(22):2461-8.

47. Trichopoulou A, Orfanos P, Norat T, Bueno-de-Mesquita B, Ocke MC, Peeters PH, et al. Modified Mediterranean diet and survival: EPIC-elderly prospective cohort study. BMJ. 2005;330(7498):991.

48. Fung TT, McCullough ML, Newby PK, Manson JE, Meigs JB, Rifai N, et al. Diet-quality scores and plasma concentrations of markers of inflammation and endothelial dysfunction. Am J Clin Nutr. 2005;82(1):163-73.

49. Buckland G, Gonzalez CA, Agudo A, Vilardell M, Berenguer A, Amiano P, et al. Adherence to the Mediterranean diet and risk of coronary heart disease in the Spanish EPIC Cohort Study. Am J Epidemiol. 2009;170(12):1518-29.

50. Zaragoza-Marti A, Cabanero-Martinez MJ, Hurtado-Sanchez JA, Laguna-Perez A, FerrerCascales R. Evaluation of Mediterranean diet adherence scores: a systematic review. BMJ Open. 2018;8(2):e019033.

51. Benitez-Arciniega AA, Mendez MA, Baena-Diez JM, Rovira Martori MA, Soler C, Marrugat $\mathrm{J}$, et al. Concurrent and construct validity of Mediterranean diet scores as assessed by an FFQ. Public Health Nutr. 2011;14(11):2015-21.

52. Knoops KT, de Groot LC, Kromhout D, Perrin AE, Moreiras-Varela O, Menotti A, et al. Mediterranean diet, lifestyle factors, and 10-year mortality in elderly European men and women: the HALE project. JAMA. 2004;292(12):1433-9.

53. Benetou V, Trichopoulou A, Orfanos P, Naska A, Lagiou P, Boffetta P, et al. Conformity to traditional Mediterranean diet and cancer incidence: the Greek EPIC cohort. Br J Cancer. 2008;99(1):191-5.

54. van den Brandt PA. The impact of a Mediterranean diet and healthy lifestyle on premature mortality in men and women. Am J Clin Nutr. 2011;94(3):913-20.

55. Buckland G, Agudo A, Travier N, Huerta JM, Cirera L, Tormo MJ, et al. Adherence to the Mediterranean diet reduces mortality in the Spanish cohort of the European Prospective Investigation into Cancer and Nutrition (EPIC-Spain). Br J Nutr. 2011;106(10):1581-91.

56. Couto E, Boffetta P, Lagiou P, Ferrari P, Buckland G, Overvad K, et al. Mediterranean dietary pattern and cancer risk in the EPIC cohort. Br J Cancer. 2011;104(9):1493-9.

57. World Cancer Research Fund / American Institute for Cancer Research. Food, nutrition, physical activity, and the prevention of cancer: a global perspective. Washington DC: American Institute for Cancer Research; 2007.

58. van den Brandt PA, Goldbohm RA, van 't Veer P, Volovics A, Hermus RJ, Sturmans F. A largescale prospective cohort study on diet and cancer in The Netherlands. J Clin Epidemiol. 1990;43(3):28595.

59. van den Brandt PA, Schouten LJ, Goldbohm RA, Dorant E, Hunen PM. Development of a record linkage protocol for use in the Dutch Cancer Registry for Epidemiological Research. Int J Epidemiol. 1990;19(3):553-8.

60. Goldbohm RA, van den Brandt PA, Brants HA, van 't Veer P, Al M, Sturmans F, et al. Validation of a dietary questionnaire used in a large-scale prospective cohort study on diet and cancer. Eur J Clin Nutr. 1994;48(4):253-65.

61. Volovics A, van den Brandt PA. Methods for the analyses of case-cohort studies. Biometrical J. 1997;39(2):195-214.

62. Boker LK, van Noord PA, van der Schouw YT, Koot NV, Bueno de Mesquita HB, Riboli E, et al. Prospect-EPIC Utrecht: study design and characteristics of the cohort population. European Prospective Investigation into Cancer and Nutrition. Eur J Epidemiol. 2001;17(11):1047-53.

63. Blokstra A, Smit HA, Bueno de Mesquita HB, Seidell JC, Verschuren WMM. Monitoring project on chronic disease risk factors (MORGEN-project) 1993-1997: prevalences and trends in lifestyle and risk, report no. 263200008. Bilthoven, the Netherlands: National Institute for Public Health and the Environment; 2005. Available from: https://www.rivm.nl/Documenten_en_publicaties/ Wetenschappelijk/Rapporten/2005/december/Monitoring_van_Risicofactoren_en_Gezondheid_in_ Nederland_MORGEN_project_1993_1997_Leefstijl_en_risicofactoren_prevalenties_en_trends (in Dutch). 
64. Beulens JW, Monninkhof EM, Verschuren WM, van der Schouw YT, Smit J, Ocke MC, et al. Cohort profile: the EPIC-NL study. Int J Epidemiol. 2010;39(5):1170-8.

65. Goldbohm RA, van 't Veer P, van den Brandt PA, van 't Hof MA, Brants HA, Sturmans F, et al. Reproducibility of a food frequency questionnaire and stability of dietary habits determined from five annually repeated measurements. Eur J Clin Nutr. 1995;49(6):420-9.

66. Prentice RL. A case-cohort design for epidemiologic cohort studies and disease prevention trials. Biometrika. 1986;73(1):1-11.

67. Ocke MC, Bueno-de-Mesquita HB, Goddijn HE, Jansen A, Pols MA, van Staveren WA, et al. The Dutch EPIC food frequency questionnaire. I. Description of the questionnaire, and relative validity and reproducibility for food groups. Int J Epidemiol. 1997;26 Suppl 1:S37-48.

68. Ocke MC, Bueno-de-Mesquita HB, Pols MA, Smit HA, van Staveren WA, Kromhout D. The Dutch EPIC food frequency questionnaire. II. Relative validity and reproducibility for nutrients. Int J Epidemiol. 1997;26 Suppl 1:S49-58.

69. NEVO table. Dutch food composition table. The Hague, the Netherlands: Voorlichtingsbureau voor de Voeding.

70. Bagnardi V, Rota M, Botteri E, Tramacere I, Islami F, Fedirko V, et al. Alcohol consumption and site-specific cancer risk: a comprehensive dose-response meta-analysis. Br J Cancer. 2015;112(3):58093. 


\section{Chapter 2}

Adherence to the Mediterranean diet and risk of lung cancer in the Netherlands Cohort Study

Maya Schulpen, Piet A. van den Brandt Br J Nutr. 2018; 119(6): 674-684 


\begin{abstract}
The evidence on a cancer-protective effect of the Mediterranean diet (MD) is still limited. Therefore, we investigated the association between MD adherence and lung cancer risk. Data were used from 120852 participants of the Netherlands Cohort Study (NLCS), aged 5569 years. Dietary habits were assessed at baseline (1986) using a validated food frequency questionnaire and alternate and modified Mediterranean diet scores (aMED and mMED, respectively), including and excluding alcohol, were calculated. After 20.3 years of followup, 2861 lung cancer cases and 3720 subcohort members (case-cohort design) could be included in multivariable Cox regression analyses. High (6-8) versus low (0-3) aMED excluding alcohol was associated with non-significantly reduced lung cancer risks in men and women with hazard ratios of 0.91 (95\% confidence interval $(\mathrm{Cl}): 0.72-1.15)$ and 0.73 (95\% Cl: $0.49-1.09)$, respectively. aMED-containing models generally fitted better than mMED-containing models. In never smokers, a borderline significant decreasing trend in lung cancer risk was observed with increasing aMED excluding alcohol. Analyses stratified by the histological lung cancer subtypes did not identify subtypes with a particularly strong inverse relation with MD adherence. Generally, the performance of aMED and WCRF/AICR dietary score variants without alcohol was comparable. In conclusion, MD adherence was non-significantly inversely associated with lung cancer risk in the NLCS. Future studies should focus on differences in associations across the sexes and histological subtypes. Furthermore, exclusion of alcohol from MD scores should be investigated more extensively, primarily with respect to a potential role of the MD in cancer prevention.
\end{abstract}




\section{Introduction}

Lung cancer was the leading cause of cancer death in the world in 2012. In that year, 1.8 million people were diagnosed, making lung cancer the most frequently diagnosed cancer in men and the third most common cancer in women worldwide [1]. The prognosis of lung cancer is poor, with five-year survival rates of $19 \%$ in the US [2] and $17 \%$ in the Netherlands [3] for lung cancers diagnosed around 2010. Tobacco smoking is the primary risk factor, but diet may influence lung cancer risk as well [4].

The traditional Mediterranean diet (MD) is a plant-based diet. Vegetables, legumes, fruits, nuts, whole grains and fish are consumed in large amounts in this dietary pattern, whereas consumption of red and processed meats, high-fat dairy products, refined grains and sweets is limited. Olive oil is the principal source of fat resulting in a relatively high ratio of monounsaturated fatty acids (MUFA) to saturated fatty acids (SFA). A final characteristic of this diet is the moderate consumption of red wine, mainly during meals [5-7]. Higher MD adherence has been associated with numerous health benefits including reduced all-cause mortality, and decreased incidence of and mortality from cardiovascular diseases [8-10]. Recently, higher MD adherence has been associated with a reduced lung cancer risk in three different cohort studies [11-13]. However, the number of lung cancer cases was small in two of the three studies, and sex-specific associations and possible differences in associations of MD adherence with the four major histological lung cancer subtypes were not investigated elaborately. Even though the association between alcohol consumption and lung cancer risk is still unclear, these previous studies included alcohol in the MD score used [14].

The present study was conducted among men and women between the ages of 55 and 69 years, who participated in the Netherlands Cohort Study (NLCS). In this paper, we aimed to investigate the association between MD adherence and the risk of lung cancer. Age-specific incidence rates of lung cancer in the Netherlands in the study period (1986-2006) were 281 per 100000 for people aged between 60 and 74 years in 1990 and 318 per 100000 for people aged 75 years or older in 2005 [3]. Additionally, we tested the heterogeneity of the association of MD adherence with lung cancer risk between the sexes and four major histological lung cancer subtypes, and evaluated the effect of exclusion of alcohol from the MD scores.

\section{Methods}

\section{Study design and lung cancer follow-up}

The NLCS is a prospective population-based cohort study that was initiated in September 1986 and has been described previously [15-18]. At enrollment, 58279 men and 62573 women, aged between 55 and 69 years, filled out a self-administered questionnaire regarding their dietary habits and other cancer risk factors. The NLCS was approved by the institutional review boards from Maastricht University and the Netherlands Organization for Applied Scientific Research. To allow efficient data processing, the nested case-cohort approach was applied, in which the number of person-years at risk is estimated based on a subcohort $(N=5000)$ that was randomly sampled just after baseline. Vital status of subcohort members was biennially verified $[15,18]$. Incident cancers in the NLCS cohort were detected by annual computerized record linkage with the Netherlands Cancer Registry and the Dutch Pathology Registry ('Pathologisch-Anatomisch Landelijk Geautomatiseerd Archief', PALGA) [16]. Reported 
computer matches, based on a linkage key, were estimated to have a sensitivity and positive predictive value of $98 \%$ [16]. Computerized linkage was followed by visual inspection in order to distinguish true matches from false positives, which maximized the positive predictive value to $100 \%$, while remaining the sensitivity unaltered [16]. It was estimated that the completeness of follow-up through record linkage with the Netherlands Cancer Registry and PALGA exceeded 95\% [19]. Incident lung cancers (International Classification of Diseases for Oncology, Third Edition, code C34) were detected until 20.3 years of follow-up. Cases with lung cancers other than carcinoma, in situ lung carcinoma or not microscopically confirmed lung cancer were excluded. Cases and subcohort members with prevalent cancer at baseline (except skin cancer), and incomplete or inconsistent (dietary) questionnaires were not eligible as well. In the end, 4084 subcohort members and 3261 lung cancer cases were eligible for inclusion in the analyses. Of the eligible study population, 2861 lung cancer cases and 3720 subcohort members were included in the Cox proportional hazards models as a result of missing values in covariates (Figure 2.1).

\section{Exposure assessment}

At baseline, NLCS participants completed a self-administered, 150-item, semi-quantitative food frequency questionnaire (FFQ) measuring dietary habits over the preceding year, which was validated by nine-day dietary records filled out over three different seasons [17]. Additionally, reproducibility of the single baseline measurement of the FFQ was investigated by means of five annually repeated measurements of dietary intake in independent random samples from the subcohort. Considering all nutrients, the average test-retest correlation of the FFQ was 0.66 . Over five years, the correlation between the baseline and repeated measurement of the FFQ had declined on average 0.07 , suggesting that the FFQ was able to rank subjects according to their nutrient intake relatively well over at least a five-year period [20]. In order to derive mean daily nutrient intakes from the questionnaire data, the 1986 Dutch food composition (NEVO) table was utilized [21].

\section{Mediterranean diet adherence}

The alternate and modified Mediterranean diet scores (aMED and mMED, respectively), which were both derived from the traditional Mediterranean diet score (tMED) developed in 1995 by Trichopoulou et al., were applied to measure relative MD adherence [22-26]. Before calculation of the aMED and $\mathrm{mMED}$, dietary intakes were standardized to a total daily energy intake of 2000 kilocalories (kcal) for women and $2500 \mathrm{kcal}$ for men [22, 26].

aMED considers the intake of nine food components, which are scored as either 0 or 1 using population-based cut-offs, resulting in a sum score varying from 0 (minimal adherence) to 9 (maximal adherence) $[25,26]$. A score of 1 is assigned to daily intakes at or above the sexspecific median for components presumed to be beneficial (vegetables (excluding potatoes), legumes, fruits, nuts, whole grains, fish, and MUFA:SFA ratio), whereas the intake of red and processed meats is scored inversely. Finally, moderate alcohol consumption (5-25 grams per day for both men and women) is assigned 1 point as well $[25,26]$.

mMED [24], developed for usage in non-Mediterranean populations, differs from aMED with regard to the food components included. In mMED, fruits and nuts are considered as one component, the cereal component includes whole and refined grains, total meat consumption is scored, dairy consumption is included as component, and the unsaturated 


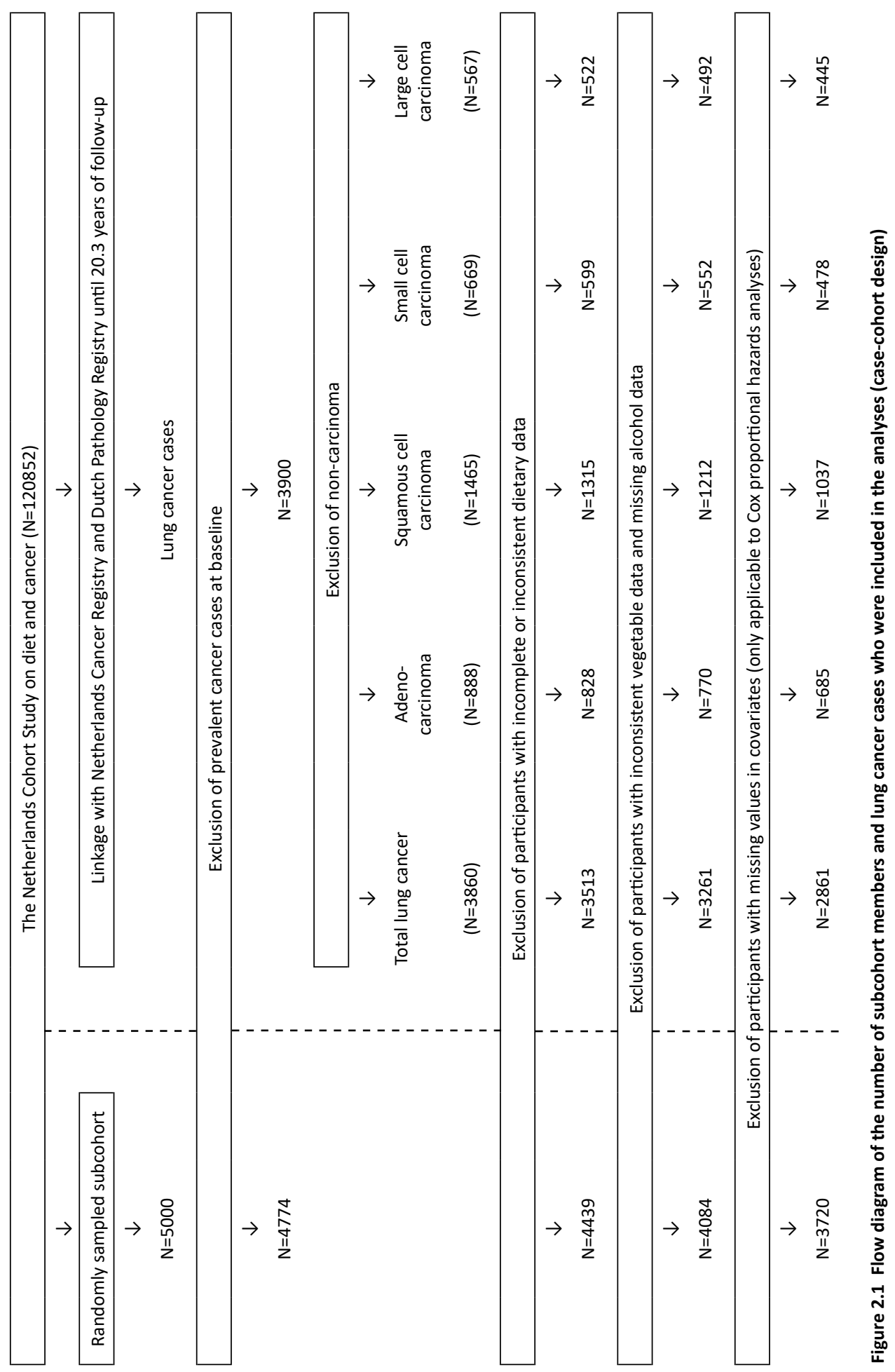


(MUFA + polyunsaturated fatty acid (PUFA)) fatty acid to SFA ratio is used. Also, the definition of moderate alcohol consumption is different (men: 10-50 grams per day; women: 5-25 grams per day) [24].

Because of the unclear association between alcohol intake and lung cancer risk [14] and variability in the definition of moderate alcohol consumption, aMED and mMED variants without alcohol were created, resulting in sum scores ranging from 0 to 8 points. We refer to these reduced scores as aMEDr and mMEDr, respectively.

\section{Statistical analyses}

Initially, all analyses were performed separately for men and women. Descriptive statistics were used to assess baseline characteristics of the subcohort.

The relation between MD adherence and lung cancer incidence was evaluated by running Cox proportional hazards models with follow-up as the timing variable to estimate hazard ratios (HRs) and 95\% confidence intervals (95\% Cls). Person-years at risk of subcohort members were calculated from baseline until lung cancer diagnosis, death, emigration, loss to follow-up, or end of follow-up, whichever occurred first. Because the variance is increased in a case-cohort design, standard errors were calculated using the Huber-White sandwich estimator [27]. Scaled Schoenfeld residuals tests, smoothed scaled Schoenfeld residuals plots and $-\ln (-\ln )$ survival plots confirmed that the proportional hazards assumption was met [28]. aMEDr and mMEDr were included in the analyses as categorical (low: 0-3; middle: 4-5; high: 6-8 points) and continuous (per 2-point increment) variables. For each exposure, three models were run, varying with respect to the covariates (literature-based) included. The age-adjusted analyses were adjusted for age at baseline (years) and sex (except sexspecific models). Additional adjustment for cigarette smoking status (never, former, current), duration (years, centered) and frequency (cigarettes smoked per day, centered) was applied in the smoking-adjusted models. The fully adjusted analyses were also adjusted for daily energy intake ( $\mathrm{kcal})$, alcohol consumption $(0,>0-<5, \geq 5-<15, \geq 15-<30, \geq 30$ grams per day), body mass index ( $\left.\mathrm{BMI},<18.5, \geq 18.5-<25, \geq 25-<30, \geq 30 \mathrm{~kg} / \mathrm{m}^{2}\right)$, non-occupational physical activity ( $\leq 30,>30-\leq 60,>60-\leq 90,>90$ minutes per day), highest level of education (primary school or lower vocational, secondary school or medium vocational, higher vocational or university), family history of lung cancer (no, yes) and history of physician-diagnosed chronic bronchitis (no, yes). Tests for trends were performed by considering the categorical MD score variables as continuous parameters.

Performances of models with aMEDr and mMEDr were compared using Akaike's Information Criterion (AIC) [29]. Additionally, AIC was used to compare the fit of models containing aMEDr and mMEDr, to the fit of models containing the original aMED and mMED including alcohol (categorized: 0-3 (low), 4-5 (middle) and 6-9 (high); continuous: per 2-point increment). The latter models did not include alcohol consumption as covariate. Based on these analyses, it was decided to assess MD adherence in the remainder of the analyses by aMEDr. Population attributable fractions (PAFs) were calculated using the STATA command punafcc, to determine the proportion of lung cancers that could be prevented if everyone had high MD adherence $[30,31]$. The effects of an alternative scenario, in which subjects with low and middle MD adherence moved one category upwards, were evaluated by means of preventable proportions (PP) [32].

The relation between MD adherence and lung cancer was also evaluated separately for the 
four major histological lung cancer subtypes: adenocarcinoma, squamous cell carcinoma, small cell carcinoma and large cell carcinoma. Heterogeneity across the subtypes was tested using a competing risks procedure. Standard errors of the difference were estimated using a bootstrapping method, especially developed for the case-cohort design, to prevent overestimation due to the absence of independence [33, 34].

Potential effect modification was evaluated by conducting Wald tests on interaction terms between the exposure of interest and age at baseline, smoking status, BMI, non-occupational physical activity, alcohol consumption and family history of lung cancer. Additionally, analyses were performed within strata of the same variables to illustrate potential modifications and to evaluate potential residual confounding by these lung cancer risk factors. Sensitivity analyses were conducted by dividing the follow-up in three periods ( $\leq 2$ years, $>2$ to $\leq 10$ years, $>10$ years) and excluding cases diagnosed during the first two years of follow-up. Moreover, the potential impact of unmeasured confounders was evaluated using rule-out approach sensitivity analysis as proposed by Schneeweiss [35].

The contribution of the individual aMEDr components to the observed associations was investigated by alternately deleting each component from the sum score using the methods described by Trichopoulou et al. [36]. Because this procedure reduces the maximum score, we multiplied the regression coefficients per 2-point increment by $8 / 9$ before exponentiating them, to obtain HRs that could be compared to effect estimates of the original score. Furthermore, the change in beneficial effect after deletion of one component was divided by the beneficial effect of the original score to acquire the percentage reduction of the inverse association. Analyses were adjusted by the subtracted component and all covariates included in the fully adjusted model.

Finally, the population-dependent assignment of scores is a potential drawback of the MD scores used, especially in non-Mediterranean populations. Therefore, we compared the performances of models containing the relative aMED variants and models containing absolute scores based on the dietary part of the recommendations to prevent cancer of the World Cancer Research Fund/American Institute for Cancer Research (WCRF/AICR) with and without alcohol [4]. We operationalized the recommendations concerning consumption of foods and drinks that promote weight gain, plant foods, red and processed meats, alcohol and salt. The absolute cut-offs used per (sub)recommendation as well as the calculation of the score have been described in detail in a previous NLCS article [37] and were based on the methods used in the EPIC cohort $[38,39]$. This resulted in a score ranging from 0 to 5 points with higher scores reflecting higher adherence to the WCRF/AICR recommendations. Analogous to aMED, we also created a WCRF/AICR score excluding the alcohol recommendation, which had a maximum value of 4 points. The continuous WCRF/ AICR score variables were included in fully adjusted Cox proportional hazards models to estimate HRs and 95\% Cls per SD increment (sex-specific). A similar approach was applied to aMED and aMEDr and the performances of models including WCRF/AICR score variables and aMED variables were compared using AIC. Analyses were carried out using STATA14 (StataCorp LP, College Station, TX, USA) and Microsoft Excel 2010 (Microsoft Corporation, Redmond, WA, USA). P-values below 0.05 were considered to be statistically significant. 


\section{Results}

After 20.3 years of follow-up, 3261 (men: 2777; women: 484) of the detected lung cancer cases met the eligibility criteria and were included in the present study. Squamous cell carcinoma (39.5\%) was the most frequently diagnosed subtype in men, followed by adenocarcinoma (21.8\%), small cell carcinoma (16.7\%) and large cell carcinoma (15.0\%). In women, these subtypes comprised $23.6 \%, 33.9 \%, 18.2 \%$ and $15.5 \%$, respectively. The remainder of the carcinomas detected was categorized as unspecified $(6.9 \%$ in men and $8.9 \%$ in women).

Subcohort members ( $50.4 \%$ male sex) had a median age of 61 years at baseline and the majority (64.9\%) was a former or current smoker. Mean values of aMEDr and mMEDr were approximately 4 in both male and female subcohort members. Sex-specific baseline characteristics of subcohort members are presented by aMEDr and mMEDr category in Table 2.1. Subcohort members with higher MD adherence were more often highly educated, more physically active and less often current smokers. Lower mean daily energy intakes were reported in both men (aMEDr and mMEDr) and women (mMEDr) with higher MD adherence. Furthermore, inverse associations with MD conformity in men were observed for alcohol consumption and having a lung cancer family history. In contrast, women with higher MD adherence were younger, consumed more alcohol (aMEDr), more often had a positive lung cancer family history (mMEDr) and less often had suffered from chronic bronchitis (aMEDr).

Because of missing values in covariates included in the multivariable analyses, 2861 lung cancer cases (men: 2413; women: 448) and 3720 subcohort members (men: 1834; women: 1886) were included in the Cox models. Sex-specific HRs and 95\% Cls for the association between MD adherence, measured by various MD scores, and lung cancer risk are displayed in Table 2.2. Statistically significant inverse associations of MD adherence with lung cancer risk were observed in all age-adjusted analyses in both sexes. After additional correction for cigarette smoking (cigarette smoking status, duration and frequency), the associations attenuated and were no longer statistically significant, but generally remained inverse. However, the association disappeared in men when conformity to MD was assessed using mMEDr. Additional adjustment for other potential confounders did not result in noticeable changes in the estimates. The inverse association between aMEDr and lung cancer risk seemed to be slightly stronger in women compared to men. However, this heterogeneity between the sexes was not statistically significant. Fully adjusted $\mathrm{HRs}(95 \% \mathrm{Cl})$ for the middle and high adherence categories, respectively, were $0.87(0.65 ; 1.15)$ and $0.73(0.49 ; 1.09)$ in women, and $0.86(0.73 ; 1.02)$ and $0.91(0.72 ; 1.15)$ in men, using the low category as reference. Judged by AIC, models containing aMEDr had a better fit than mMEDr-containing models. Furthermore, model fits were worse when alcohol consumption was included in the score. Therefore, as was already mentioned in the Methods section, aMEDr was used to assess MD adherence in the remainder of the analyses. Assuming causality, PAFs showed that $18.9 \%$ (95\% Cl: $-12.6 \%$ to $41.6 \%)$ and $2.0 \%$ (95\% Cl: $-19.1 \%$ to $19.4 \%$ ) of the lung cancers in women and men, respectively, would be prevented if the entire population had high MD adherence according to aMEDr. A more realistic scenario in which subjects with low and middle aMEDr values moved one category upwards would prevent $12.1 \%$ of the female and $4.0 \%$ of the male lung cancer cases, respectively.

Table 2.3 displays the results of subtype-specific Cox regression analyses. Although there seemed to be some variations in the strength of the associations across the subtypes, 
particularly in men, heterogeneity tests were not statistically significant. In men, aMEDr appeared to be non-significantly inversely associated with small cell carcinoma risk, but not with adenocarcinoma risk. Middle MD adherence was associated with (borderline) significantly reduced risks of squamous and large cell carcinoma in men. In women, higher aMEDr values were associated with non-significantly reduced risks of adenocarcinoma, squamous cell carcinoma and small cell carcinoma.

Both sexes were combined in the subgroup analyses (Supplementary Table S2.1) to increase the power, since there was no evidence of heterogeneity. Stratification by smoking status showed a borderline statistically significant decreasing trend in lung cancer risk with increasing aMEDr in never smokers $(p=0.07)$, but not in former $(p=0.38)$ or current smokers $(p=0.28)$. However, the interaction test was not statistically significant $\left(p_{\text {heterogeneity }}=0.07\right)$. The inverse association between MD adherence and lung cancer risk seemed to be limited to subjects with a normal BMI ( $p_{\text {heterogeneity }}=0.03$ ) and those who consumed 15 grams or more alcohol per day $\left(p_{\text {heterogeneity }}=0.02\right)$. Associations were similar across strata of age at baseline, level of non-occupational physical activity, lung cancer family history and duration of followup. In addition, exclusion of cases diagnosed during the first two years of follow-up did not result in noteworthy changes of the effect estimates.

Rule-out approach sensitivity analyses showed that an unmeasured confounder, which increases lung cancer risk three times, would need to be 3.3 and 2.5 times more common in women and men with low MD adherence, respectively, to be able to explain the observed HRs (Supplementary Figure S2.1). A population prevalence of $10 \%$ for the confounder was assumed to obtain these results.

The contribution of the individual aMEDr components to the observed HRs was evaluated and results are listed in Table 2.4. After exclusion of nuts from the score, the inverse association completely disappeared in men. Furthermore, a fully adjusted model including all aMEDr components as dichotomous variables showed that nut intake was statistically significantly associated with a reduced lung cancer risk in men (data not shown). Intakes of fruits and vegetables also considerably contributed to the inverse relation in men. Removal of one of these components weakened the strength of the relation by almost $50 \%$. The inverse association in men gained strength when any of the other components was excluded from the score. In women, intakes of fruits and whole grains, and to a lesser extent the MUFA:SFA ratio, were most strongly associated with a reduced lung cancer risk.

We also compared performances of the relative aMED indices and the absolute WCRF/AICR scores (Table 2.5). HRs were estimated per SD increment to ensure comparability. Both scores showed inverse associations (mostly not statistically significant) with lung cancer risk in men and women, independently of whether alcohol consumption was taken into account in the sum score. HRs $(95 \% \mathrm{CI})$ for the WCRF/AICR score without alcohol were $0.96(0.88$; $1.04)$ and $0.92(0.80 ; 1.07)$ in men and women, respectively. When the WCRF/AICR score including alcohol was considered, we observed a statistically significant inverse association with lung cancer risk in men (HR per SD increment, $95 \% \mathrm{Cl}: 0.90,0.83$ to 0.98 ), but not women. The similar AIC values indicated comparable fits of models containing WCRF/AICR score and aMED variables without alcohol in both sexes. 


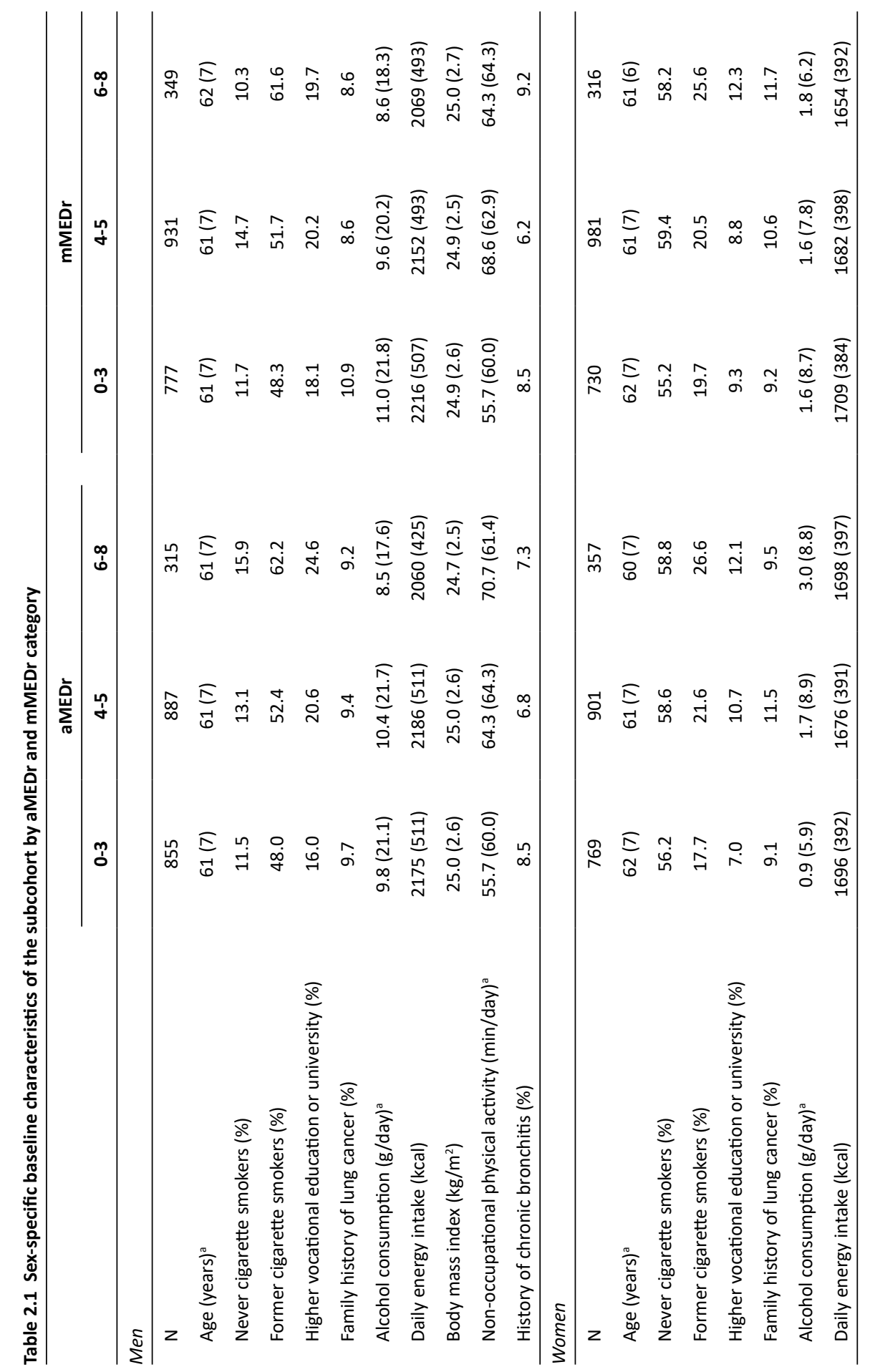




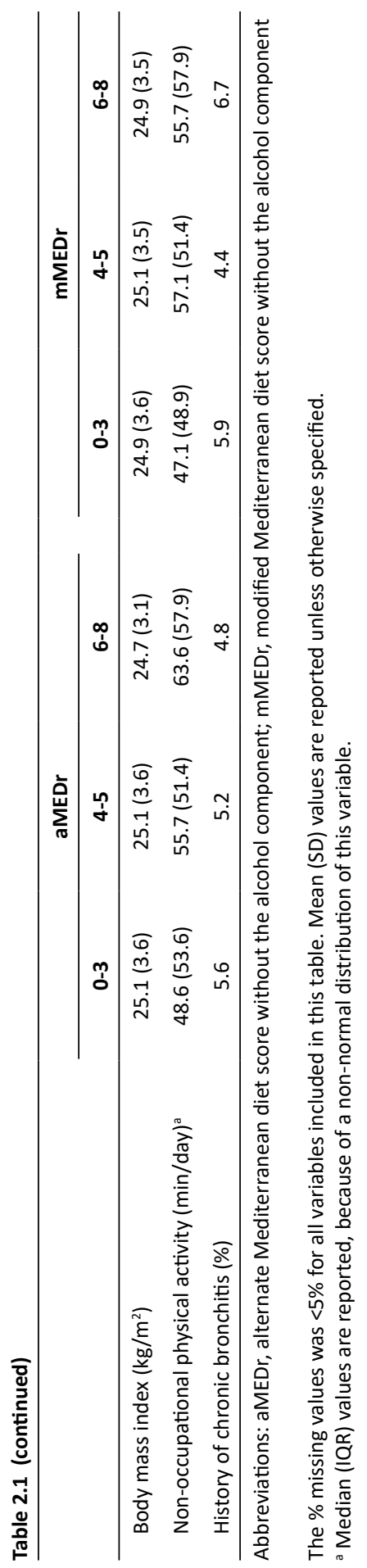




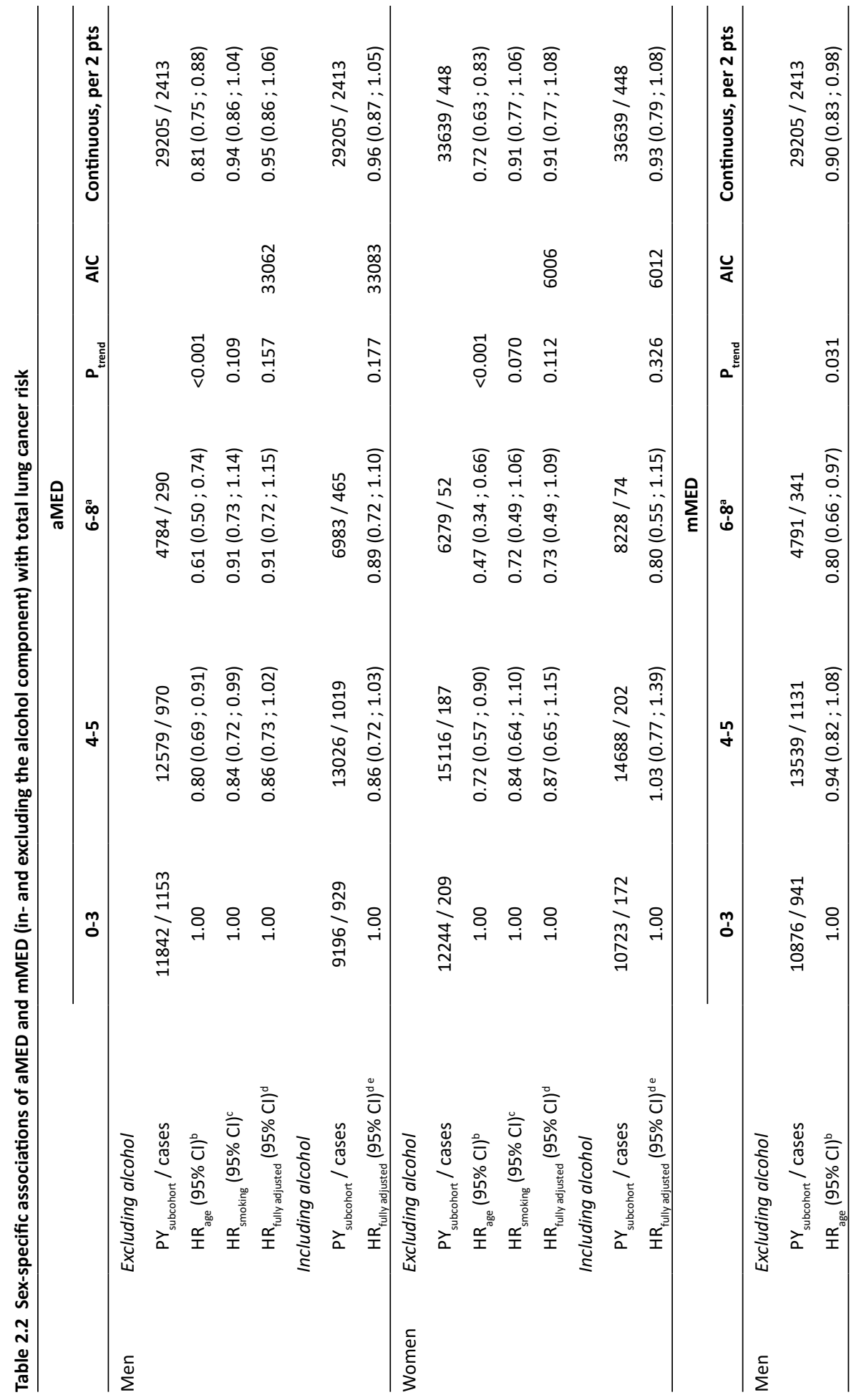




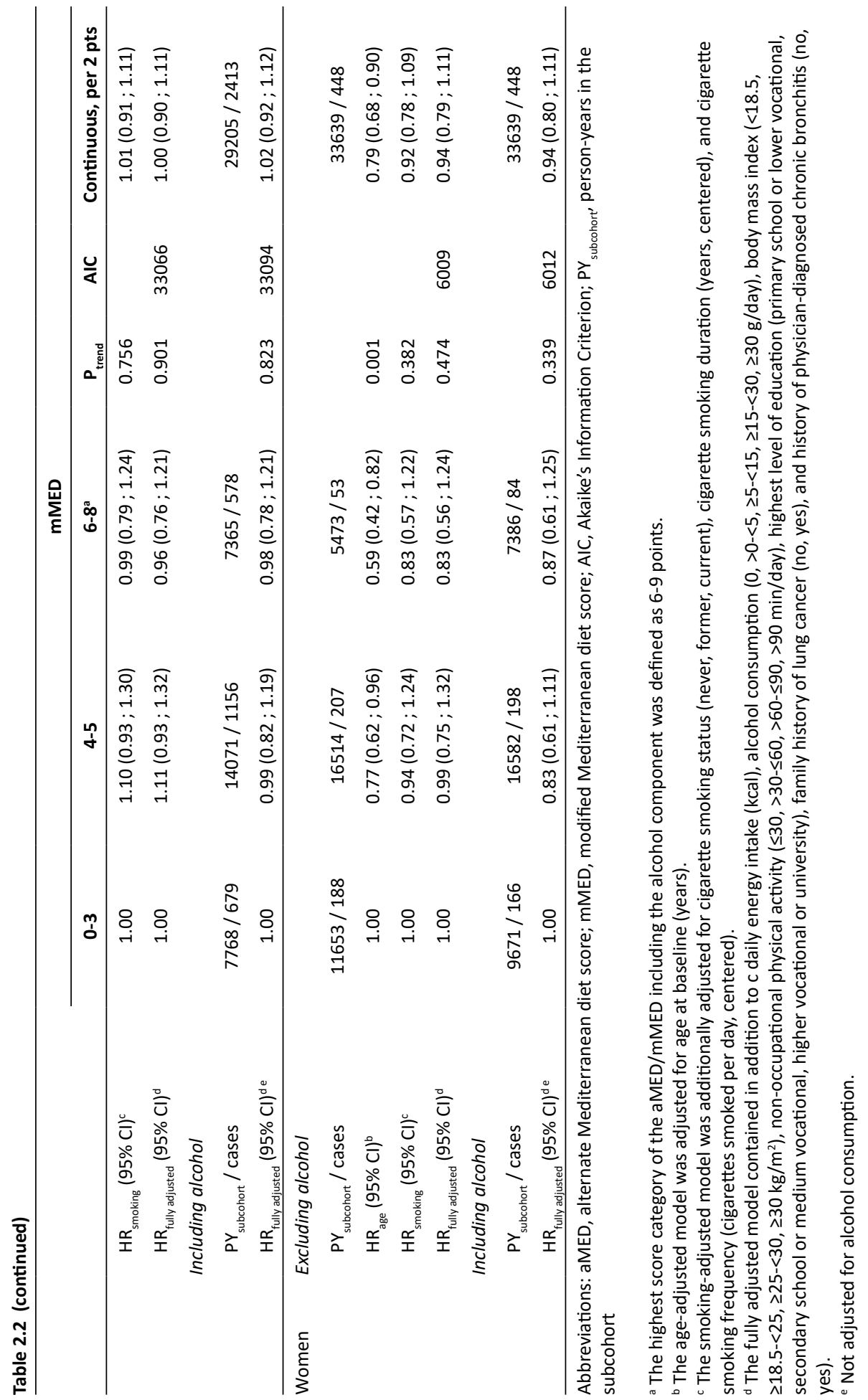




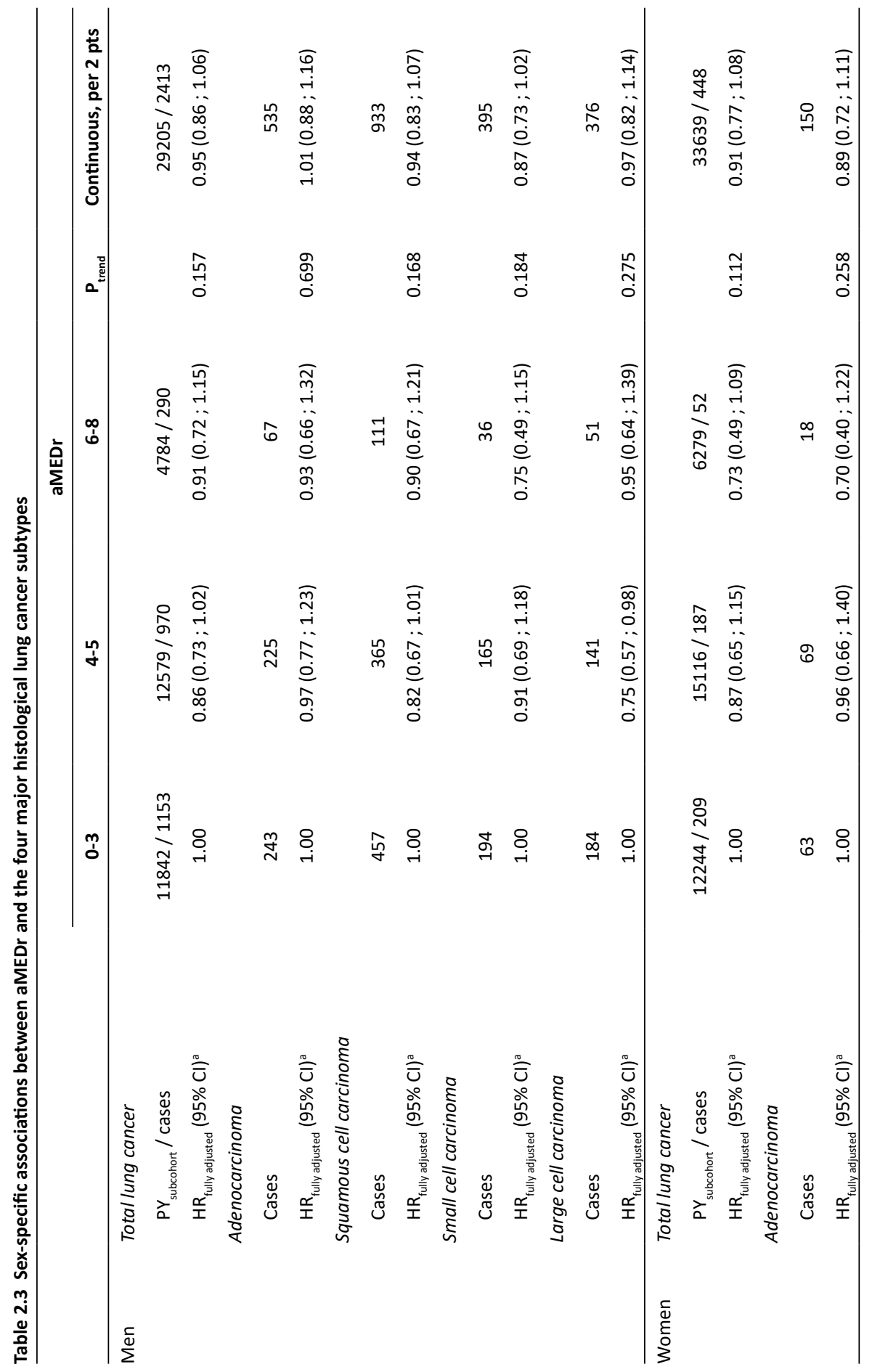




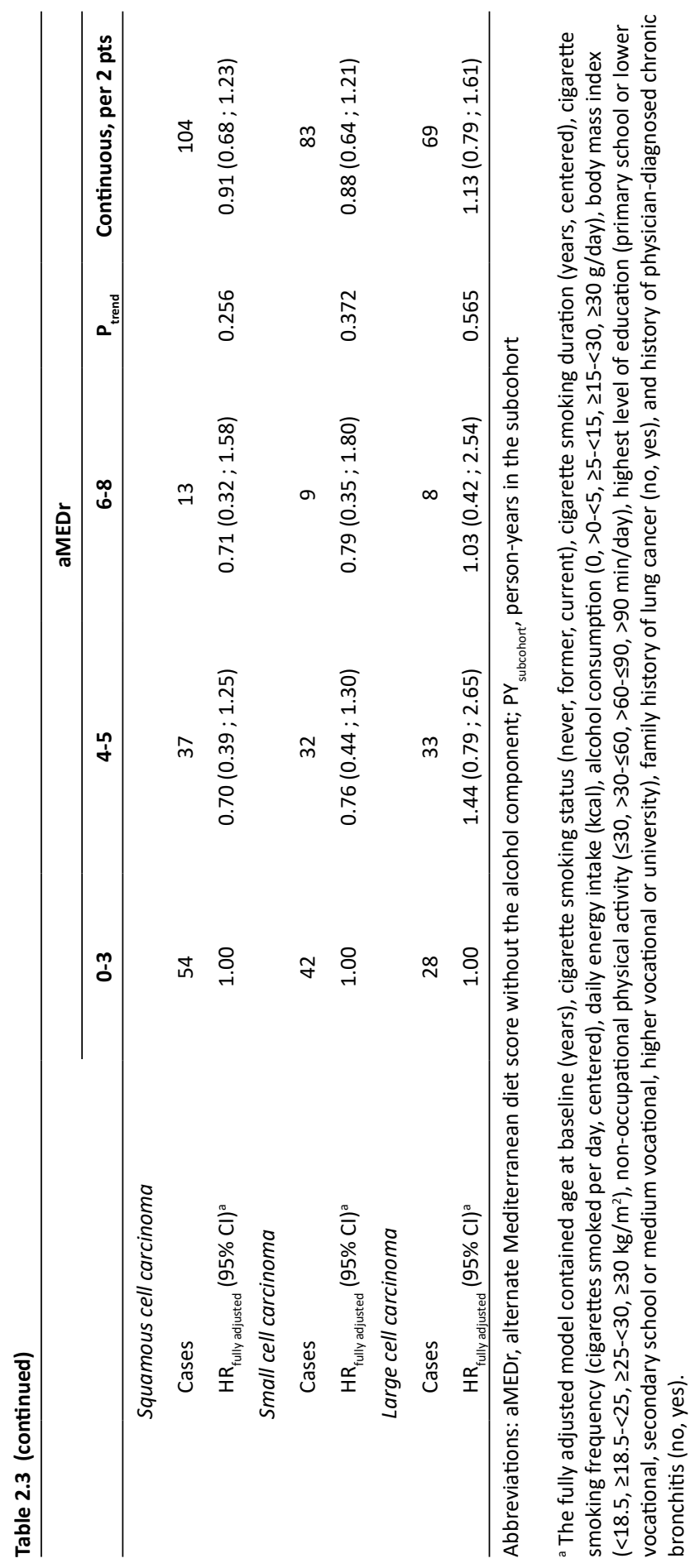




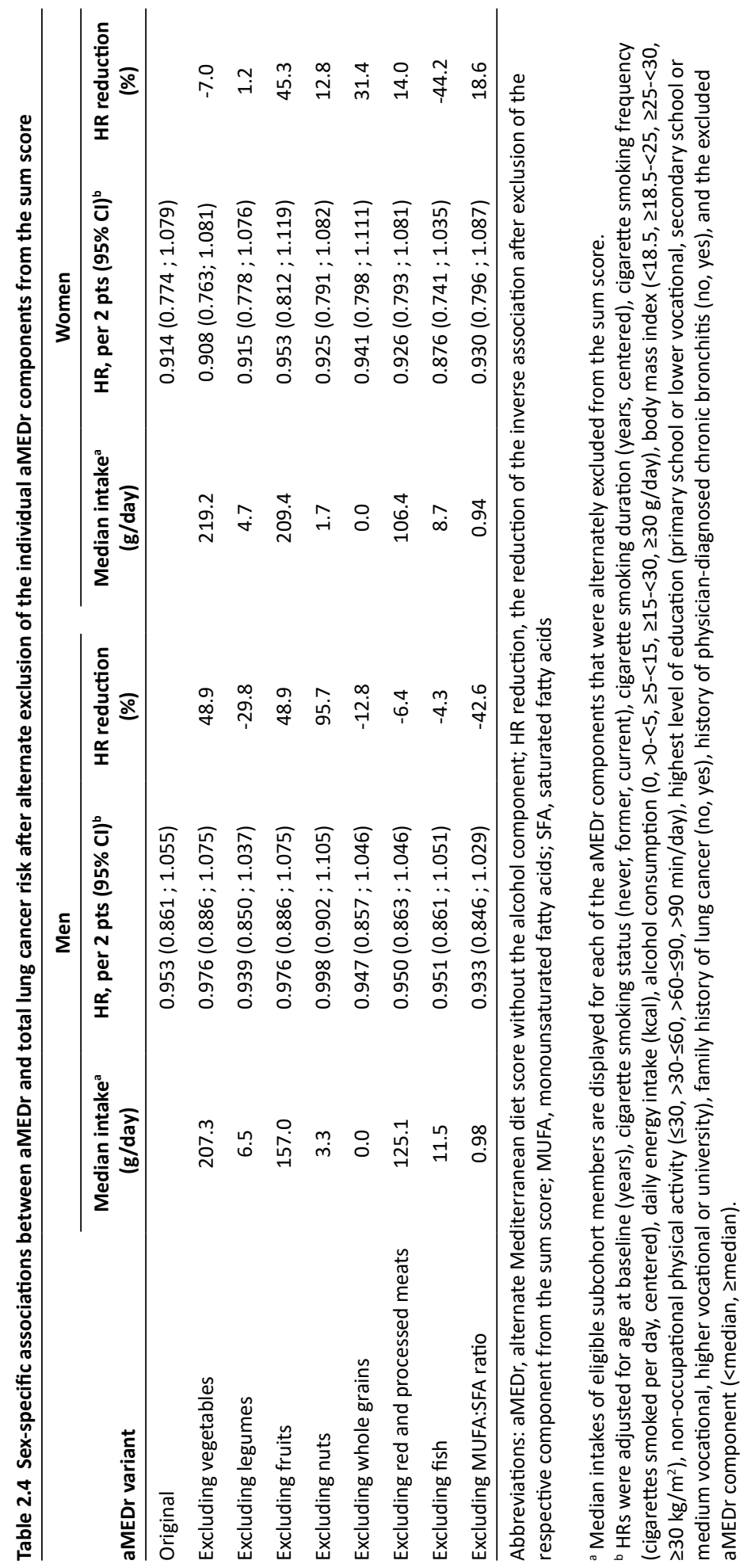


Table 2.5 Sex-specific associations of the absolute WCRF/AICR diet score and aMED (per SD-increment) with total lung cancer risk

\begin{tabular}{|c|c|c|c|c|}
\hline & \multicolumn{2}{|l|}{ Men } & \multicolumn{2}{|l|}{ Women } \\
\hline & $\mathrm{HR}_{\mathrm{SD}}(95 \% \mathrm{Cl})^{\mathrm{a} b}$ & AIC & $\mathrm{HR}_{\mathrm{SD}}(95 \% \mathrm{Cl})^{\mathrm{a} b}$ & AIC \\
\hline $\mathrm{PY}_{\text {subcohort }} /$ cases $^{\mathrm{c}}$ & $28564 / 2341$ & & $32992 / 441$ & \\
\hline \multicolumn{5}{|l|}{ Excluding alcohol } \\
\hline WCRF/AICR diet score ${ }^{d}$ & $0.96(0.88 ; 1.04)$ & 31960 & $0.92(0.80 ; 1.07)$ & 5895 \\
\hline aMEDr & $0.96(0.89 ; 1.05)$ & 31961 & $0.94(0.83 ; 1.08)$ & 5896 \\
\hline \multicolumn{5}{|l|}{ Including alcohol } \\
\hline WCRF/AICR diet score ${ }^{\mathrm{d} e}$ & $0.90(0.83 ; 0.98)$ & 31960 & $0.94(0.81 ; 1.08)$ & 5902 \\
\hline aMEDe & $0.97(0.89 ; 1.05)$ & 31980 & $0.94(0.83 ; 1.07)$ & 5902 \\
\hline
\end{tabular}

Abbreviations: WCRF/AICR, World Cancer Research Fund/American Institute for Cancer Research; aMED, alternate Mediterranean diet score; AIC, Akaike's Information Criterion; PY $_{\text {subcohort }}$ person-years in the subcohort; aMEDr, alternate Mediterranean diet score without the alcohol component

\footnotetext{
${ }^{a}$ HRs were estimated per sex-specific SD-increment in the scores.

${ }^{b}$ HRs were adjusted for age at baseline (years), cigarette smoking status (never, former, current), cigarette smoking duration (years, centered), cigarette smoking frequency (cigarettes smoked per day, centered), daily energy intake (kcal), alcohol consumption $(0,>0-<5, \geq 5-<15, \geq 15-<30, \geq 30 \mathrm{~g} /$ day), body mass index $(<18.5$, $\left.\geq 18.5-<25, \geq 25-<30, \geq 30 \mathrm{~kg} / \mathrm{m}^{2}\right)$, non-occupational physical activity $(\leq 30,>30-\leq 60,>60-\leq 90,>90 \mathrm{~min} /$ day), highest level of education (primary school or lower vocational, secondary school or medium vocational, higher vocational or university), family history of lung cancer (no, yes), and history of physician-diagnosed chronic bronchitis (no, yes).

${ }^{c}$ A lower number of subjects could be included in these analyses as a result of missing values for salt intake.

d Score based on WCRF/AICR dietary recommendations to prevent cancer.

e Not adjusted for alcohol consumption.
}

\section{Discussion}

Higher MD adherence was associated with a non-significantly reduced lung cancer risk in the NLCS. HRs $(95 \% \mathrm{Cl})$ for comparisons of high to low aMEDr values were $0.73(0.49 ; 1.09)$ in women and $0.91(0.72 ; 1.15)$ in men. Associations seemed to vary across the histological subtypes, though heterogeneity tests were not statistically significant. A borderline statistically significant inverse trend was observed in never smokers when the sexes were combined. In our study population, the performance of aMED was superior to that of mMED and better model fits were obtained when alcohol intake was not included in the score.

The results of the present study were in line with those of previous prospective cohort studies [11-13]. Nonetheless, in contrast to these studies, the fully adjusted inverse associations observed in the present study lacked statistical significance. In the NIH-AARP study, a HR of 0.85 ( $95 \% \mathrm{Cl}: 0.79$ to 0.91 ) for lung cancer risk was observed when comparing the highest to the lowest aMED quintile [12]. An Australian study estimated a HR of 0.64 (95\% Cl: 0.45 to 0.90) for high compared to low MD adherence, based on an adapted version of tMED [13]. Similarly, an inverse association was found in an Italian study among (former) heavy smokers [11]. HRs varied between 0.58 and 0.66 when comparing aMED values of 2-3, 4-5 and 6-7 to a value of $0-1$. A substantial reduction in lung cancer risk (90\%) was associated with having an aMED value of $8-9$, but this was only based on one lung cancer case. 
When we combined men and women, we found a HR of 0.89 (95\% $\mathrm{Cl}: 0.73$ to 1.10) for high versus low MD adherence (aMEDr). Compared to our study, the inverse associations observed in previously conducted cohort studies were stronger [11-13]. This difference might be attributed to higher proportions of Mediterranean subjects, particularly in the Australian study $[13,40]$, which deliberately oversampled Italian and Greek migrants (approximately one-quarter of the cohort), and the Italian study [11], possibly resulting in larger contrasts in absolute food intakes between subjects with high and low MD adherence. Furthermore, wide intake distributions were noted in the NIH-AARP cohort [41]. Methods used to adjust for cigarette smoking in the NIH-AARP study and the Australian study seemed to be less comprehensive compared to the method used in the present study, increasing the likelihood that residual confounding by cigarette smoking could have impacted the results of these studies. The NIH-AARP cohort did not take the duration of smoking into account [12], while smoking duration and frequency were combined into the composite measure "pack-years" in the Australian study [13]. Nevertheless, non-significant inverse associations between MD adherence and lung cancer risk were present in never smokers in both studies, which is similar to observations in male and female NLCS participants who never smoked. Additionally, in never smoking NLCS participants, non-significant inverse associations were observed with all histological subtypes. Therefore, we consider a large impact of residual confounding by cigarette smoking on the results of our study unlikely.

Sex-specific associations of MD adherence with lung cancer risk have seldom been reported in prior studies. Only the NIH-AARP study showed sex-specific associations and reported a statistically significant inverse relation between MD adherence and lung cancer risk in both sexes [12]. In our study, MD adherence seemed to have a slightly stronger inverse relation with lung cancer risk in women compared to men. Because this heterogeneity was not statistically significant and data from previous research is mostly lacking, additional research on sex-specific associations between MD adherence and lung cancer risk is required before any conclusions can be drawn regarding this topic.

The association of MD adherence with the histological lung cancer subtypes has rarely been evaluated as well. Higher MD adherence was statistically significantly associated with a reduced risk of adenocarcinoma and squamous cell carcinoma, but not small cell carcinoma, in the NIH-AARP cohort [12]. Although the heterogeneity tests were not statistically significant, results of our study suggested that associations with MD adherence might vary across the lung cancer subtypes, particularly in men. However, the currently available evidence is not sufficient to conclude anything concerning possible variations in associations of the lung cancer subtypes with MD adherence.

Results of the rule-out approach sensitivity analyses suggested that a strong unmeasured lung cancer risk factor with appreciable inequalities in its distribution over the MD adherence categories would be required to fully explain the observed associations. Occupational exposure to asbestos, for instance, substantially increases lung cancer risk [42]. However, it is questionable whether its association with MD adherence is sufficiently strong and we did adjust for level of education. Other lung cancer risk factors that remained unmeasured were assumed to generally be weak. So, it is unlikely that unmeasured confounders have substantially impacted our study results.

A previous cohort study showed that, of the components included in aMEDr, whole grains and fruits had the strongest inverse relation with both male and female lung cancer risk [12]. We reported similar results with respect to female lung cancer risk. However, nut 
intake seemed to be the most important driver of the inverse association observed in male NLCS participants. Some individual components were not associated with lung cancer risk or had associations in unexpected directions, emphasizing the potential advantages of analysis of dietary patterns as opposed to individual dietary components. Dietary pattern analysis takes into account that individual dietary components may interact synergistically or antagonistically while exerting their effects on health. Additionally, weak and otherwise undetectable effects of singular components may become detectable if they are combined in dietary patterns. Furthermore, foods are generally consumed in certain patterns, raising collinearity and confounding issues when individual components are evaluated. Finally, by grouping dietary components together, contrasts in healthiness of the diet within the study population will probably increase, thereby increasing the chance of detecting true effects, if present $[23,43,44]$.

Evidence on the relation between alcohol consumption and lung cancer risk is inconclusive [14]. Therefore, we excluded alcohol from the MD scores and evaluated the effect of this exclusion. Both the present and a previous NLCS analysis showed better performances of models using MD scores without alcohol [37]. However, earlier cohort studies regarding $\mathrm{MD}$ adherence and lung cancer risk all considered moderate alcohol consumption as a beneficial component of the MD score used. Similar to a previous NLCS analysis concerning breast cancer risk, model performances were better when aMED variants were included compared to mMED variants [37]. aMED variants consider whole grains as a beneficial component, whereas whole grains and refined grains are combined in mMED variants, despite their potentially differential health effects [45]. Similarly, mMED variants include total meat consumption, while aMED variants consider the intake of red and processed meats, specifically [11,46-48]. These and other differences in the composition of the food components included might explain the better performance of aMED variants in our study population.

Reduced levels of reactive oxygen species-induced DNA damage, oxidative stress, and inflammation may mediate the potential cancer-protective effect of the MD [44, 49]. Consumption of vegetables, legumes, fruits, nuts, whole grains and olive oil is high in the plant-based MD $[5,6]$. These foods are rich in dietary fiber, vitamins, minerals and polyphenols and have been associated with high levels of antioxidants and low levels of oxidized LDL-cholesterol [44, 49-54]. Furthermore, polyphenols may exert anti-inflammatory effects [55].

It is a potential weakness that the interpretation of the value of aMEDr depends on the study population, particularly in a non-Mediterranean population. Therefore, we compared the performance of this score to that of an absolute WCRF/AICR score. Generally, the performance of the aMEDr and the WCRF/AICR score without alcohol was comparable. The WCRF/AICR score without alcohol showed a non-significant and weak inverse association with lung cancer risk in both sexes. A SD increment in WCRF/AICR score including alcohol was statistically significantly associated with a reduced lung cancer risk in men, but not women. Higher adherence to the WCRF/AICR recommendations has previously been associated with a reduced lung cancer risk in the EPIC cohort (HR per one-point increment, $95 \% \mathrm{Cl}: 0.92$, 0.89 to 0.96 ) [38]. In contrast, a WCRF/AICR score was not associated with lung cancer risk in the elderly according to a meta-analysis within the CHANCES consortium (HR per one-point increment, $95 \% \mathrm{Cl}: 0.99,0.84$ to 1.17 ) [56], indicating that additional research is warranted.

The NLCS cohort has a high follow-up completion rate, which minimized the risk of 
information and selection bias. Additionally, the large number of lung cancer cases allowed extensive adjustment for potential confounding, subtype-specific analyses and analyses stratified by smoking status. A potential weakness of the NLCS is the single assessment of dietary habits and potential confounders at baseline, which may have resulted in attenuated associations. Also, measurement errors in the assessment of dietary intake cannot fully be excluded and might have resulted in misclassification. The dependence of aMEDr on the study population is a final potential weakness. Because our study was conducted in a nonMediterranean population, one may question the extent to which high values of aMEDr reflected a truly Mediterranean way of eating.

Though the inverse associations lacked statistical significance, the present study is in agreement with other cohort studies showing that high MD adherence might be associated with a reduced lung cancer risk and suggested that this inverse association might also be generalizable to non-Mediterranean populations. Additionally, we observed potential differences in associations between the sexes and histological subtypes warranting future research. Finally, exclusion of alcohol from MD scores should be investigated more extensively, primarily with respect to a potential role of the MD in cancer prevention. 


\section{References}

1. Ferlay J, Soerjomataram I, Ervik M, Dikshit R, Eser S, Mathers C, et al. GLOBOCAN 2012 v1.0, Cancer Incidence and Mortality Worldwide: IARC CancerBase No. 11 [Internet]. Lyon, France: International Agency for Research on Cancer; 2013 [cited 2016 July]. Available from: http://globocan. iarc.fr.

2. Howlader N, Noone AM, Krapcho M, Miller D, Bishop K, Altekruse SF, et al. SEER Cancer Statistics Review, 1975-2013. Bethesda, MD: National Cancer Institute; 2016 [cited 2017 February]. Available from: http://seer.cancer.gov/csr/1975_2013/, based on November 2015 SEER data submission, posted to the SEER web site, April 2016.

3. Nederlandse Kankerregistratie, operated by IKNL: IKNL [cited 2017 February]. Available from: http://www.cijfersoverkanker.nl/.

4. World Cancer Research Fund / American Institute for Cancer Research. Food, nutrition, physical activity, and the prevention of cancer: a global perspective. Washington DC: American Institute for Cancer Research; 2007.

5. Willett WC, Sacks F, Trichopoulou A, Drescher G, Ferro-Luzzi A, Helsing E, et al. Mediterranean diet pyramid: a cultural model for healthy eating. Am J Clin Nutr. 1995;61(6 Suppl):1402S-6S.

6. Trichopoulou A, Lagiou P. Healthy traditional Mediterranean diet: an expression of culture, history, and lifestyle. Nutr Rev. 1997;55(11 Pt 1):383-9.

7. Fung TT, Rexrode KM, Mantzoros CS, Manson JE, Willett WC, Hu FB. Mediterranean diet and incidence of and mortality from coronary heart disease and stroke in women. Circulation. 2009;119(8):1093-100.

8. Sofi F, Macchi C, Abbate R, Gensini GF, Casini A. Mediterranean diet and health status: an updated meta-analysis and a proposal for a literature-based adherence score. Public Health Nutr. 2014;17(12):2769-82.

9. Martinez-Gonzalez MA, Bes-Rastrollo M. Dietary patterns, Mediterranean diet, and cardiovascular disease. Curr Opin Lipidol. 2014;25(1):20-6.

10. Grosso G, Marventano S, Yang J, Micek A, Pajak A, Scalfi L, et al. A comprehensive metaanalysis on evidence of Mediterranean diet and cardiovascular disease: Are individual components equal? Crit Rev Food Sci Nutr. 2017;57(15):3218-32.

11. Gnagnarella P, Maisonneuve P, Bellomi M, Rampinelli C, Bertolotti R, Spaggiari L, et al. Red meat, Mediterranean diet and lung cancer risk among heavy smokers in the COSMOS screening study. Ann Oncol. 2013;24(10):2606-11.

12. Anic GM, Park Y, Subar AF, Schap TE, Reedy J. Index-based dietary patterns and risk of lung cancer in the NIH-AARP diet and health study. Eur J Clin Nutr. 2016;70(1):123-9.

13. Hodge AM, Bassett JK, Shivappa N, Hebert JR, English DR, Giles GG, et al. Dietary inflammatory index, Mediterranean diet score, and lung cancer: a prospective study. Cancer Causes Control. 2016;27(7):907-17.

14. International Agency for Research on Cancer. IARC Monographs on the evaluation of carcinogenic risks to humans: alcohol consumption and ethyl carbamate, vol. 96. Lyon, France: International Agency for Research on Cancer; 2010.

15. van den Brandt PA, Goldbohm RA, van 't Veer P, Volovics A, Hermus RJ, Sturmans F. A largescale prospective cohort study on diet and cancer in The Netherlands. J Clin Epidemiol. 1990;43(3):28595.

16. van den Brandt PA, Schouten LJ, Goldbohm RA, Dorant E, Hunen PM. Development of a record linkage protocol for use in the Dutch Cancer Registry for Epidemiological Research. Int J Epidemiol. 1990;19(3):553-8.

17. Goldbohm RA, van den Brandt PA, Brants HA, van 't Veer P, Al M, Sturmans F, et al. Validation of a dietary questionnaire used in a large-scale prospective cohort study on diet and cancer. Eur J Clin Nutr. 1994;48(4):253-65.

18. Volovics A, van den Brandt PA. Methods for the analyses of case-cohort studies. Biometrical J. 1997;39(2):195-214.

19. van den Brandt PA, van 't Veer P, Goldbohm RA, Dorant E, Volovics A, Hermus RJ, et al. A prospective cohort study on dietary fat and the risk of postmenopausal breast cancer. Cancer Res. 1993;53(1):75-82. 
20. Goldbohm RA, van 't Veer P, van den Brandt PA, van ' $t$ Hof MA, Brants HA, Sturmans F, et al. Reproducibility of a food frequency questionnaire and stability of dietary habits determined from five annually repeated measurements. Eur J Clin Nutr. 1995;49(6):420-9.

21. NEVO table. Dutch food composition table 1986-1987. The Hague, the Netherlands: Voorlichtingsbureau voor de Voeding; 1986.

22. Trichopoulou A, Kouris-Blazos A, Wahlqvist ML, Gnardellis C, Lagiou P, Polychronopoulos E, et al. Diet and overall survival in elderly people. BMJ. 1995;311(7018):1457-60.

23. Trichopoulou A, Costacou T, Bamia C, Trichopoulos D. Adherence to a Mediterranean diet and survival in a Greek population. N Engl J Med. 2003;348(26):2599-608.

24. Trichopoulou A, Orfanos P, Norat T, Bueno-de-Mesquita B, Ocke MC, Peeters PH, et al. Modified Mediterranean diet and survival: EPIC-elderly prospective cohort study. BMJ. 2005;330(7498):991.

25. Fung TT, McCullough ML, Newby PK, Manson JE, Meigs JB, Rifai N, et al. Diet-quality scores and plasma concentrations of markers of inflammation and endothelial dysfunction. Am J Clin Nutr. 2005;82(1):163-73.

26. Mitrou PN, Kipnis V, Thiebaut AC, Reedy J, Subar AF, Wirfalt E, et al. Mediterranean dietary pattern and prediction of all-cause mortality in a US population: results from the NIH-AARP Diet and Health Study. Arch Intern Med. 2007;167(22):2461-8.

27. Lin DY, Wei LJ. The Robust Inference for the Cox Proportional Hazards Model. J Am Stat Assoc. 1989;84(408):1074-8.

28. Grambsch PM, Therneau TM. Proportional Hazards Tests and Diagnostics Based on Weighted Residuals. Biometrika. 1994;81(3):515-26.

29. Akaike H. A new look at the statistical model identification. IEEE Transactions on automatic control. 1974;AC-19(6):716-23.

30. Rockhill B, Newman B, Weinberg C. Use and misuse of population attributable fractions. Am J Public Health. 1998;88(1):15-9.

31. Newson RB. Attributable and unattributable risks and fractions and other scenario comparisons. SJ. 2013;13:672-98.

32. Wahrendorf J. An estimate of the proportion of colo-rectal and stomach cancers which might be prevented by certain changes in dietary habits. Int J Cancer. 1987;40(5):625-8.

33. Wacholder S, Gail MH, Pee D, Brookmeyer R. Alternative Variance and Efficiency Calculations for the Case-Cohort Design. Biometrika. 1989;76(1):117-23.

34. de Vogel S, Bongaerts BW, Wouters KA, Kester AD, Schouten LJ, de Goeij AF, et al. Associations of dietary methyl donor intake with MLH1 promoter hypermethylation and related molecular phenotypes in sporadic colorectal cancer. Carcinogenesis. 2008;29(9):1765-73.

35. Schneeweiss S. Sensitivity analysis and external adjustment for unmeasured confounders in epidemiologic database studies of therapeutics. Pharmacoepidemiol Drug Saf. 2006;15(5):291-303.

36. Trichopoulou A, Bamia C, Trichopoulos D. Anatomy of health effects of Mediterranean diet: Greek EPIC prospective cohort study. BMJ. 2009;338:b2337.

37. van den Brandt PA, Schulpen M. Mediterranean diet adherence and risk of postmenopausal breast cancer: results of a cohort study and meta-analysis. Int J Cancer. 2017;140(10):2220-31.

38. Romaguera D, Vergnaud AC, Peeters PH, van Gils CH, Chan DS, Ferrari P, et al. Is concordance with World Cancer Research Fund/American Institute for Cancer Research guidelines for cancer prevention related to subsequent risk of cancer? Results from the EPIC study. Am J Clin Nutr. 2012;96(1):150-63.

39. Vergnaud AC, Romaguera D, Peeters PH, van Gils CH, Chan DS, Romieu I, et al. Adherence to the World Cancer Research Fund/American Institute for Cancer Research guidelines and risk of death in Europe: results from the European Prospective Investigation into Nutrition and Cancer cohort study. Am J Clin Nutr. 2013;97(5):1107-20.

40. Harriss LR, English DR, Powles J, Giles GG, Tonkin AM, Hodge AM, et al. Dietary patterns and cardiovascular mortality in the Melbourne Collaborative Cohort Study. Am J Clin Nutr. 2007;86(1):2219.

41. Schatzkin A, Subar AF, Thompson FE, Harlan LC, Tangrea J, Hollenbeck AR, et al. Design and serendipity in establishing a large cohort with wide dietary intake distributions : the National Institutes of Health-American Association of Retired Persons Diet and Health Study. Am J Epidemiol. 2001;154(12):1119-25. 
42. Ngamwong $\mathrm{Y}$, Tangamornsuksan $\mathrm{W}$, Lohitnavy $\mathrm{O}$, Chaiyakunapruk N, Scholfield CN, Reisfeld $B$, et al. Additive Synergism between Asbestos and Smoking in Lung Cancer Risk: A Systematic Review and Meta-Analysis. PLoS One. 2015;10(8):e0135798.

43. Jacques PF, Tucker KL. Are dietary patterns useful for understanding the role of diet in chronic disease? Am J Clin Nutr. 2001;73(1):1-2.

44. Verberne L, Bach-Faig A, Buckland G, Serra-Majem L. Association between the Mediterranean diet and cancer risk: a review of observational studies. Nutr Cancer. 2010;62(7):860-70.

45. Aune D, Chan DS, Lau R, Vieira R, Greenwood DC, Kampman E, et al. Dietary fibre, whole grains, and risk of colorectal cancer: systematic review and dose-response meta-analysis of prospective studies. BMJ. 2011;343:d6617.

46. Chan DS, Lau R, Aune D, Vieira R, Greenwood DC, Kampman E, et al. Red and processed meat and colorectal cancer incidence: meta-analysis of prospective studies. PLoS One. 2011;6(6):e20456.

47. Yang WS, Wong MY, Vogtmann E, Tang RQ, Xie L, Yang YS, et al. Meat consumption and risk of lung cancer: evidence from observational studies. Ann Oncol. 2012;23(12):3163-70.

48. Shi Y, Yu PW, Zeng DZ. Dose-response meta-analysis of poultry intake and colorectal cancer incidence and mortality. Eur J Nutr. 2015;54(2):243-50.

49. Brill JB. The Mediterranean diet and your health. Am J Lifestyle Med. 2009;3(1):44-56.

50. Ferro-Luzzi A, Branca F. Mediterranean diet, Italian-style: prototype of a healthy diet. Am J Clin Nutr. 1995;61(6 Suppl):1338S-45S.

51. Kushi LH, Lenart EB, Willett WC. Health implications of Mediterranean diets in light of contemporary knowledge. 1. Plant foods and dairy products. Am J Clin Nutr. 1995;61(6 Suppl):1407S$15 \mathrm{~s}$.

52. Marrugat J, Covas MI, Fito M, Schroder H, Miro-Casas E, Gimeno E, et al. Effects of differing phenolic content in dietary olive oils on lipids and LDL oxidation--a randomized controlled trial. Eur J Nutr. 2004;43(3):140-7.

53. Pitsavos C, Panagiotakos D, Trichopoulou A, Chrysohoou C, Dedoussis G, Chloptsios Y, et al. Interaction between Mediterranean diet and methylenetetrahydrofolate reductase C677T mutation on oxidized low density lipoprotein concentrations: the ATTICA study. Nutr Metab Cardiovasc Dis. 2006;16(2):91-9.

54. Bogani P, Visioli F. Antioxidants in the Mediterranean diets: An update. World Rev Nutr Diet. 2007;97:162-79.

55. Rahman I, Biswas SK, Kirkham PA. Regulation of inflammation and redox signaling by dietary polyphenols. Biochem Pharmacol. 2006;72(11):1439-52.

56. Jankovic N, Geelen A, Winkels RM, Mwungura B, Fedirko V, Jenab M, et al. Adherence to the WCRF/AICR Dietary Recommendations for Cancer Prevention and Risk of Cancer in Elderly from Europe and the United States: A Meta-Analysis within the CHANCES Project. Cancer Epidemiol Biomarkers Prev. 2017;26(1):136-44. 
Chapter 2

\section{Supplementary materials}

Supplementary Table S2.1 Fully adjusted associations ${ }^{a}$ (men and women combined) between aMEDr and total lung cancer risk in various subgroups

\begin{tabular}{|c|c|c|c|c|c|c|}
\hline Subgroup & & $0-3$ & $4-5$ & $6-8$ & $\mathbf{P}_{\text {trend }}$ & $\mathbf{P}_{\text {heterogeneity }}{ }^{\mathbf{b}}$ \\
\hline \multicolumn{7}{|l|}{ Baseline age } \\
\hline \multirow[t]{2}{*}{$55-59$ years } & Cases & 511 & 424 & 116 & & \\
\hline & $\mathrm{HR}(95 \% \mathrm{Cl})$ & 1.00 & $0.98(0.77 ; 1.25)$ & $0.92(0.64 ; 1.30)$ & 0.667 & \\
\hline \multirow[t]{2}{*}{$60-64$ years } & Cases & 471 & 407 & 126 & & \\
\hline & $\mathrm{HR}(95 \% \mathrm{Cl})$ & 1.00 & $0.78(0.60 ; 1.00)$ & $0.87(0.61 ; 1.23)$ & 0.137 & \\
\hline \multirow[t]{2}{*}{$65-69$ years } & Cases & 380 & 326 & 100 & & \\
\hline & $\mathrm{HR}(95 \% \mathrm{Cl})$ & 1.00 & $0.78(0.58 ; 1.06)$ & $0.95(0.63 ; 1.42)$ & 0.356 & 0.564 \\
\hline \multicolumn{7}{|l|}{ Smoking status ${ }^{c}$} \\
\hline \multirow[t]{2}{*}{ Never } & Cases & 82 & 88 & 28 & & \\
\hline & $\mathrm{HR}(95 \% \mathrm{Cl})$ & 1.00 & $0.95(0.67 ; 1.35)$ & $0.60(0.35 ; 1.01)$ & 0.072 & \\
\hline \multirow[t]{2}{*}{ Former } & Cases & 363 & 390 & 133 & & \\
\hline & $\mathrm{HR}(95 \% \mathrm{Cl})$ & 1.00 & $1.08(0.85 ; 1.36)$ & $0.82(0.61 ; 1.11)$ & 0.382 & \\
\hline \multirow[t]{2}{*}{ Current } & Cases & 917 & 679 & 181 & & \\
\hline & $\mathrm{HR}(95 \% \mathrm{Cl})$ & 1.00 & $0.79(0.64 ; 0.97)$ & $1.02(0.73 ; 1.43)$ & 0.280 & 0.074 \\
\hline \multicolumn{7}{|l|}{ Body mass index ${ }^{d}$} \\
\hline \multirow[t]{2}{*}{$\geq 18.5-<25.0 \mathrm{~kg} / \mathrm{m}^{2}$} & Cases & 763 & 619 & 184 & & \\
\hline & $\mathrm{HR}(95 \% \mathrm{Cl})$ & 1.00 & $0.74(0.61 ; 0.91)$ & $0.72(0.54 ; 0.95)$ & 0.003 & \\
\hline \multirow[t]{2}{*}{$\geq 25.0 \mathrm{~kg} / \mathrm{m}^{2}$} & Cases & 593 & 521 & 156 & & \\
\hline & $\mathrm{HR}(95 \% \mathrm{Cl})$ & 1.00 & $0.99(0.80 ; 1.23)$ & $1.10(0.80 ; 1.51)$ & 0.702 & 0.030 \\
\hline \multicolumn{7}{|l|}{ Physical activitye } \\
\hline \multirow[t]{2}{*}{$\leq 30 \mathrm{~min} /$ day } & Cases & 337 & 226 & 56 & & \\
\hline & $\mathrm{HR}(95 \% \mathrm{Cl})$ & 1.00 & $0.99(0.71 ; 1.39)$ & $1.05(0.61 ; 1.81)$ & 0.919 & \\
\hline \multirow[t]{2}{*}{$>30-\leq 60 \mathrm{~min} / \mathrm{day}$} & Cases & 413 & 351 & 108 & & \\
\hline & $\mathrm{HR}(95 \% \mathrm{Cl})$ & 1.00 & $0.83(0.63 ; 1.09)$ & $1.03(0.71 ; 1.49)$ & 0.586 & \\
\hline \multirow[t]{2}{*}{$>60-\leq 90 \mathrm{~min} / \mathrm{day}$} & Cases & 238 & 231 & 75 & & \\
\hline & $\mathrm{HR}(95 \% \mathrm{Cl})$ & 1.00 & $1.08(0.74 ; 1.59)$ & $0.70(0.42 ; 1.15)$ & 0.285 & \\
\hline \multirow[t]{2}{*}{$>90 \mathrm{~min} /$ day } & Cases & 374 & 349 & 103 & & \\
\hline & $\mathrm{HR}(95 \% \mathrm{Cl})$ & 1.00 & $0.75(0.58 ; 0.98)$ & $0.78(0.54 ; 1.11)$ & 0.048 & 0.526 \\
\hline \multicolumn{7}{|l|}{ Alcohol consumption ${ }^{f}$} \\
\hline \multirow[t]{2}{*}{$0 \mathrm{~g} /$ day } & Cases & 224 & 157 & 49 & & \\
\hline & $\mathrm{HR}(95 \% \mathrm{Cl})$ & 1.00 & $0.91(0.64 ; 1.30)$ & $1.04(0.64 ; 1.70)$ & 0.892 & \\
\hline \multirow[t]{2}{*}{$>0-<15.0 \mathrm{~g} /$ day } & Cases & 498 & 521 & 161 & & \\
\hline & $\mathrm{HR}(95 \% \mathrm{Cl})$ & 1.00 & $1.13(0.90 ; 1.42)$ & $1.05(0.77 ; 1.43)$ & 0.494 & \\
\hline \multirow[t]{2}{*}{$\geq 15.0 \mathrm{~g} / \mathrm{day}$} & Cases & 640 & 479 & 132 & & \\
\hline & $\mathrm{HR}(95 \% \mathrm{Cl})$ & 1.00 & $0.64(0.50 ; 0.81)$ & $0.75(0.53 ; 1.04)$ & 0.002 & 0.016 \\
\hline
\end{tabular}


Supplementary Table S2.1 (continued)

\begin{tabular}{|c|c|c|c|c|c|c|}
\hline Subgroup & & $0-3$ & $4-5$ & $6-8$ & $\mathbf{P}_{\text {trend }}$ & $\mathbf{P}_{\text {heterogeneity }}{ }^{\mathbf{b}}$ \\
\hline \multicolumn{7}{|l|}{$\begin{array}{l}\text { Lung cancer family } \\
\text { history }^{\mathrm{g}}\end{array}$} \\
\hline \multirow[t]{2}{*}{ No } & Cases & 1177 & 1013 & 300 & & \\
\hline & HR (95\% Cl) & 1.00 & $0.89(0.76 ; 1.05)$ & $0.89(0.72 ; 1.10)$ & 0.155 & \\
\hline \multirow[t]{2}{*}{ Yes } & Cases & 185 & 144 & 42 & & \\
\hline & $\mathrm{HR}(95 \% \mathrm{Cl})$ & 1.00 & $0.67(0.41 ; 1.09)$ & $0.70(0.32 ; 1.53)$ & 0.153 & 0.438 \\
\hline \multicolumn{7}{|l|}{ Follow-up } \\
\hline \multirow[t]{2}{*}{$0-\leq 2$ years } & Cases & 121 & 94 & 22 & & \\
\hline & $\mathrm{HR}(95 \% \mathrm{Cl})$ & 1.00 & $0.88(0.64 ; 1.21)$ & $0.67(0.41 ; 1.10)$ & 0.113 & \\
\hline \multirow[t]{2}{*}{$>2-\leq 10$ years } & Cases & 604 & 508 & 148 & & \\
\hline & $\mathrm{HR}(95 \% \mathrm{Cl})$ & 1.00 & $0.90(0.75 ; 1.08)$ & $0.92(0.71 ; 1.19)$ & 0.316 & \\
\hline \multirow[t]{2}{*}{$>10-20.3$ years } & Cases & 637 & 555 & 172 & & \\
\hline & $\mathrm{HR}(95 \% \mathrm{Cl})$ & 1.00 & $0.83(0.70 ; 1.00)$ & $0.91(0.71 ; 1.18)$ & 0.152 & 0.678 \\
\hline
\end{tabular}

Abbreviation: aMEDr, alternate Mediterranean diet score without the alcohol component

${ }^{a}$ HRs were adjusted for age at baseline (years), sex (men, women), cigarette smoking status (never, former, current), cigarette smoking duration (years, centered), cigarette smoking frequency (cigarettes smoked per day, centered), daily energy intake ( $\mathrm{kcal})$, alcohol consumption $(0,>0-<5, \geq 5-<15, \geq 15-<30, \geq 30 \mathrm{~g} /$ day), body mass index $\left(<18.5, \geq 18.5-<25, \geq 25-<30, \geq 30 \mathrm{~kg} / \mathrm{m}^{2}\right)$, non-occupational physical activity $(\leq 30,>30-\leq 60,>60-\leq 90$, $>90 \mathrm{~min} /$ day), highest level of education (primary school or lower vocational, secondary school or medium vocational, higher vocational or university), family history of lung cancer (no, yes), and history of physiciandiagnosed chronic bronchitis (no, yes).

${ }^{\mathrm{b}}$ P-values for heterogeneity were obtained by fitting interaction terms between aMEDr and the stratifying covariates in fully adjusted models.

${ }^{c}$ Not adjusted for cigarette smoking status.

${ }^{\mathrm{d}}$ Not adjusted for body mass index.

e Not adjusted for non-occupational physical activity.

${ }^{f}$ Not adjusted for alcohol consumption.

g Not adjusted for family history of lung cancer. 
(A)

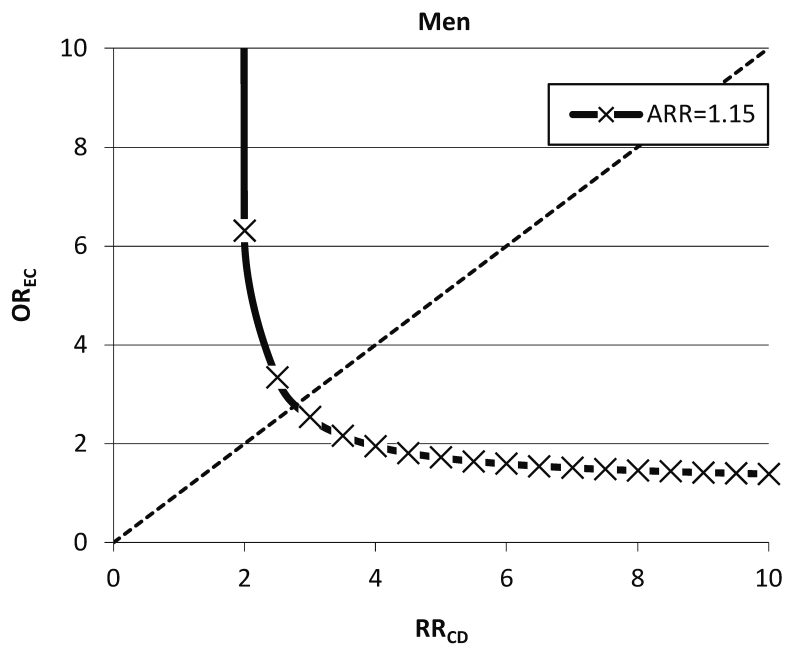

(B)

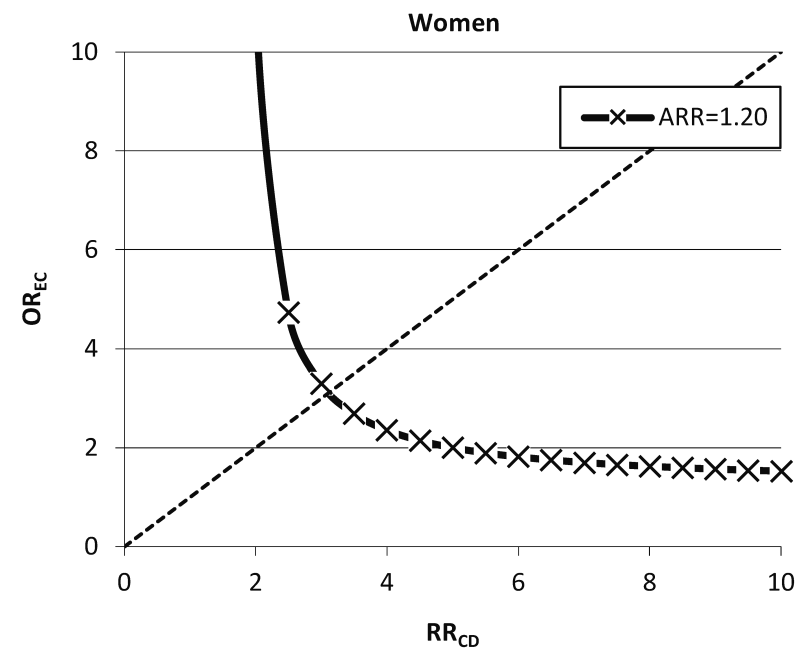

Supplementary Figure S2.1 Results of the rule-out approach sensitivity analyses in men (A) and women (B) to evaluate the potential impact of unmeasured confounders

Unmeasured confounders with $\mathrm{OR}_{\mathrm{EC}}$ and $\mathrm{RR}_{\mathrm{CD}}$ combinations that lie in the upper right areas above the curves are sufficiently strong to cause us to find the observed ARR in case of a 'true' hazard ratio of 1.00.

Note: aMEDr was dichotomized into low (0-3) and middle/high (4-8) scores in order to perform the rule-out approach sensitivity analyses. ARR was calculated using middle/high aMEDr values as reference. Prevalences of exposure (low aMEDr) were $41 \%$ in men and $37 \%$ in women, respectively, whereas a confounder prevalence of $10 \%$ was assumed.

Abbreviations: $A R R$, apparent relative risk; $\mathrm{OR}_{\mathrm{EC}}$, odds ratio exposure-confounder association; $\mathrm{RR}_{\mathrm{CD}}$, relative risk confounder-disease association; aMEDr, alternate Mediterranean diet score without the alcohol component 
Mediterranean diet and lung cancer risk 


\section{Chapter 3}

\section{Mediterranean diet adherence and risk of postmenopausal breast cancer: results of a cohort study and meta-analysis}

Piet A. van den Brandt, Maya Schulpen Int J Cancer. 2017; 140(10): 2220-2231 


\section{Abstract}

The Mediterranean diet (MD) has been associated with reduced mortality and risk of cardiovascular diseases, but there is only limited evidence on cancer.

We investigated the relationship between adherence to MD and risk of postmenopausal breast cancer (and estrogen/progesterone receptor subtypes, ER/PR). In the Netherlands Cohort Study, 62573 women aged 55-69 years provided information on dietary and lifestyle habits in 1986. Follow-up for cancer incidence until 2007 (20.3 years) consisted of record linkages with the Netherlands Cancer Registry and the Dutch Pathology Registry PALGA. Adherence to MD was estimated through the alternate Mediterranean Diet Score excluding alcohol. Multivariate case-cohort analyses were based on 2321 incident breast cancer cases and 1665 subcohort members with complete data on diet and potential confounders. We also conducted meta-analyses of our results with those of other published cohort studies.

We found a statistically significant inverse association between MD adherence and risk of ER negative (ER-) breast cancer, with a hazard ratio (HR) of 0.60 (95\% confidence interval: $0.39,0.93$ ) for high versus low $M D$ adherence ( $p$-trend=0.032). MD adherence showed only non-significant weak inverse associations with ER positive (ER+) or total breast cancer risk. In meta-analyses, summary HRs for high versus low MD adherence were 0.94 for total postmenopausal breast cancer, 0.98 for ER+, 0.73 for ER- and 0.77 for ER-PR- breast cancer. Our findings support an inverse association between MD adherence and, particularly, receptor negative breast cancer. This may have important implications for prevention because of the poorer prognosis of these breast cancer subtypes. 


\section{Introduction}

Breast cancer is the most commonly diagnosed cancer in Western countries, and prevention is of paramount importance to reduce the burden of this disease. Thus far, very few modifiable (lifestyle) risk factors, such as overweight and alcohol consumption, have been identified. Intake of individual dietary factors has been extensively studied in relation to breast cancer risk, but only for alcohol there is convincing evidence for an increased risk [1]. Because individuals do not consume isolated foods or nutrients, studying dietary patterns in relation to breast cancer seems more fruitful, thereby acknowledging interactions between individual components as well as existing collinearity between components. Dietary patterns might also yield more actionable information on dietary change needed for prevention.

In contrast with dietary patterns that are a posteriori derived from factor or cluster analyses of a dataset, the Mediterranean diet (MD) score is a dietary quality index, a priori constructed on the basis of dietary recommendations [2]. The traditional MD is characterized by a high intake of plant proteins, whole grains, fish, and monounsaturated fat, moderate alcohol intake and low intake of refined grains, red meat, and sweets $[3,4]$. MD adherence is associated with decreased risk of mortality and cardiovascular diseases; however, for cancer risk, results are still rather limited. A recent meta-analysis [5] reported a lower incidence of overall breast cancer for women adhering to the highest category of MD-scores in case-control studies, but not in cohort studies. It is important to distinguish between pre- and postmenopausal breast cancer, as well as hormone receptor subtypes, because of differences in etiology. The meta-analysis suggested that evidence for an inverse association with MD was more convincing for postmenopausal breast cancer. Furthermore, differences were noted between different estrogen/progesterone receptor (ER/PR) subtypes of breast cancer in the associations with $\mathrm{MD}$, but this observation was based on very few cohort studies. Recent evidence from a randomized controlled trial (RCT) on primary prevention of cardiovascular diseases indicated a strong protective effect of MD on the risk of postmenopausal breast cancer in Spain [6].

We investigated the association between adherence to MD and postmenopausal breast cancer risk, overall and stratified by hormone receptor status, in the Netherlands Cohort Study (NLCS). Based on earlier findings [7], we hypothesized that MD adherence would show a stronger inverse association with ER negative (ER-) breast cancer than ER positive $(E R+)$ breast cancer, which may have important implications for prevention because of the poorer prognosis of ER- breast cancer. Because alcohol is a risk factor for breast cancer [8], we excluded it from the MD-score that normally includes moderate alcohol consumption, and tested the effect of this exclusion. We also conducted meta-analyses on MD adherence and breast cancer risk by subtype.

\section{Materials and methods}

\section{Study design and cancer follow-up}

The NLCS started in September 1986 and the female part included 62573 women aged 5569 years [9]. At baseline, participants completed a mailed, self-administered questionnaire on cancer risk factors. The NLCS study was approved by institutional review boards from Maastricht University and the Netherlands Organization for Applied Scientific Research. All cohort members consented to participation by completing the questionnaire. For data 
processing and analysis the case-cohort method was used [10]. Accumulated person-years in the cohort were estimated from a subcohort ( $N=2589$ women), randomly sampled from the cohort immediately after baseline. These subcohort members were actively followed up biennially for vital status information. The follow-up of the subcohort was $100 \%$ complete at 20.3 years of follow-up.

Follow-up for cancer incidence was established by annual record linkage with the Netherlands Cancer Registry and PALGA, the nationwide Dutch Pathology Registry [11]. Completeness of follow-up through record linkage with cancer registries and PALGA was estimated to be greater than 95\% [12]. After 20.3 years of follow-up (September 17, 1986 until January 1, 2007), a total of 3354 incident breast cancer cases were detected among women. Cases and subcohort members were excluded if they reported a history of cancer (except skin cancer) at baseline and if their dietary data were incomplete or inconsistent. Supplementary Figure S3.1 shows the selection and exclusion steps that resulted in the number of cases and female subcohort members that were included in the analysis. There were 1665 subcohort members and 2321 breast cancer cases available for analysis.

\section{Exposure assessment}

The 11-page baseline questionnaire measured dietary intake, detailed smoking habits, anthropometry, physical activity and other risk factors related to cancer [9]. Habitual consumption of food and beverages during the year preceding baseline was assessed using a 150 -item semi-quantitative food frequency questionnaire, which was validated against a nine-day diet record [13]. Nutrient intakes were calculated using the computerized Dutch food composition table [14]. Non-occupational physical activity was calculated by adding the minutes spent per day on cycling or walking, shopping, walking the dog, gardening, and sports or exercise as reported previously [15].

\section{Mediterranean diet score}

Conformity with the MD was assessed using the alternate Mediterranean Diet Score (aMED) $[16,17]$, which is an adapted version of the traditional Mediterranean Diet Score created by Trichopoulou et al. $[18,19]$. The aMED contains nine dietary components that are typical of the MD. To control for energy intake, the intake of each component was first adjusted to a daily intake of $2000 \mathrm{kcal}[16,17,19]$. For each of the presumed beneficial food items (vegetables (without potatoes), legumes, fruits, nuts, whole grains, fish, and the ratio of monounsaturated to saturated fatty acid intake (MUFA:SFA)), 1 point was given when the intake was at least the sex-specific median intake, and 0 otherwise. For red and processed meat, 1 point was given (and 0 otherwise) when the intake was below the sex-specific median intake. In the full aMED, 1 additional point is normally given when alcohol intake is between 5-25 g/day, and 0 otherwise [17]. However, since alcohol is a risk factor for breast cancer [8], we excluded alcohol from the score in the present analysis. The reduced sum score (aMEDr) ranged from 0 to 8 points (minimal to maximal conformity). MD adherence was also assessed using the modified Mediterranean Diet Score by Trichopoulou et al. [20], abbreviated as mMED. Apart from alcohol, this score differs from aMED as follows: fruits and nuts are combined in one component; dairy is considered as component; cereals are considered as component instead of whole grains; total meat is used instead of red and processed meat; and for fatty acids the ratio of unsaturated (MUFA + polyunsaturated fatty acids (PUFA)) fatty acids to SFA is used. 


\section{Statistical analysis}

The reduced scores (aMEDr and mMEDr) were categorized in three categories: 0-3, 4-5, and 6-8 points. The distribution of the subcohort members by aMEDr-score, mMEDr-score and various characteristics was examined by cross-tabulations and summary statistics.

The relationship between MD adherence and breast cancer risk was evaluated using Cox proportional hazards models. It was verified that the proportional hazards assumption was not violated using scaled Schoenfeld residuals [21] and $-\ln (-\ln )$ survival plots. Standard errors were estimated using the robust Huber-White sandwich estimator to account for additional variance introduced by the subcohort sampling [22]. We conducted age- and multivariableadjusted survival analyses in which aMEDr and mMEDr were tested on categorical and continuous scales. In the multivariable analyses, hazard ratios (HRs) were corrected for potential confounding by age at baseline (55-59, 60-64, 65-69 years), cigarette smoking (status (never, former, current), frequency (number of cigarettes per day; continuous, centered), duration (number of years; continuous, centered)), body height (continuous, $\mathrm{cm})$, body mass index (BMl; $\left.<18.5,18.5-<25,25-<30, \geq 30 \mathrm{~kg} / \mathrm{m}^{2}\right)$, non-occupational physical activity ( $\leq 30,>30-60,>60-90,>90 \mathrm{~min} /$ day), highest level of education (primary school or lower vocational, secondary school or medium vocational, and higher vocational or university), family history of breast cancer in mother or sisters (no, yes), history of benign breast disease (no, yes), age at menarche $(\leq 12,13-14,15-16, \geq 17$ years), parity (nulliparous, $1-2, \geq 3$ children), age at first birth $(<25, \geq 25$ years), age at menopause $(<45,45-49,50-54$, $\geq 55$ years), oral contraceptive use (never, ever), postmenopausal hormone replacement therapy (never, ever), energy intake (continuous, kcal/day), and, depending on the analysis, alcohol intake $(0,0.1-<5,5-<15,15-<30, \geq 30 \mathrm{~g} /$ day).

Tests for trends were assessed by fitting ordinal exposure variables as continuous terms.

The Akaike Information Criterion (AIC) [23] was used to compare performance of models with aMEDr- and mMEDr-scores. We also analyzed associations with the full aMED- and mMED-scores (including alcohol) to compare these with models using aMEDr and mMEDr, using the AIC. Besides overall postmenopausal breast cancer, we conducted these analyses for subtypes defined by hormone receptor status: ER+, ER-, PR+, PR-, ER+PR+, and ER-PR-. Differences in associations with MD-scores between breast cancer subtypes were tested using a heterogeneity test [24], in which the standard error for the observed difference in rate ratios was estimated using a bootstrapping method developed for the case-cohort design [25]. To evaluate potential residual confounding by breast cancer risk factors, and effect modification, analyses of MD-scores and breast cancer were also conducted within strata of age at baseline, smoking status, alcohol intake, BMI, physical activity, and family history of breast cancer. Interactions with these factors were tested using Wald tests and cross-product terms. In sensitivity analyses, we repeated analyses after excluding cancers (and person-years) occurring in the first two years of follow-up, and we also split the followup period in three periods.

Population attributable fractions (PAFs) were calculated [26] to estimate the potentially avoidable proportion of cancer if all participants would shift towards the highest MD-score category. As a more realistic scenario, preventable proportions were also calculated to estimate the preventable proportion of cancer if all participants in the lowest two categories of MD-scores would shift their pattern one category upward [27, 28]. The Stata-command "punafcc" was used to calculate the PAFs and 95\% confidence intervals (95\% Cls) [29]. 
To investigate possible dominance of certain components of the MD-scores [30], we ran analyses in which all components were entered simultaneously as dichotomous variables in Cox regression models. We then alternately subtracted one component at a time from the original 9-point sum score (thus reducing it to an 8-point score), and estimated HRs per 2-point increment in the reduced score (corrected by $8 / 9$ before exponentiating them to preserve comparability), as in Trichopoulou et al. [30].

The MD-scores are relative measures, using cohort-specific medians as cut-offs. We compared the MD-score findings with a score that uses absolute cut-offs, based on the dietary part of the cancer prevention recommendations issued by World Cancer Research Fund/ American Institute for Cancer Research (WCRF/AICR) [1]. We operationalized their dietary recommendations by using the same absolute cut-offs per recommendation (sometimes subrecommendations) as in the European Prospective Investigation into Cancer and Nutrition (EPIC) cohort [31, 32], using scores of 1 if the recommendation was met, 0.5 if half met, and 0 if not met. This concerned intake of energy-dense foods $(\leq 125,>125-<175, \geq 175 \mathrm{kcal} / 100$ g per day) and sugary drinks $(0, \leq 250,>250 \mathrm{~g} /$ day); vegetables and fruits $(\geq 400,200-<400$, $<200 \mathrm{~g} /$ day), and dietary fiber ( $\geq 25,12.5-<25,<12.5 \mathrm{~g} /$ day); red and processed meat (red \& processed meat $<500 \mathrm{~g} /$ week and processed meat $<3 \mathrm{~g} /$ day, red \& processed meat $<500 \mathrm{~g} /$ week and processed meat 3- $<50 \mathrm{~g} /$ day, red \& processed meat $\geq 500 \mathrm{~g} /$ week or processed meat $\geq 50 \mathrm{~g} /$ day); and alcohol $(<10,10-20,>20 \mathrm{~g} /$ day). We additionally operationalized the WCRF/AICR-recommendation on salt intake by categorizing the calculated [33] total salt intake (from food and salt added during cooking or consumption) into $<6,6-<9,9+\mathrm{g} /$ day with scores 1, 0.5, and 0, respectively (based on Dutch dietary guidelines 2015 [34]). The resulting sum score (ranging from 0 to 5 ) was used in survival analyses; an additional sum score without alcohol was also made. The AIC was used to compare the fit of models with these sum scores to models with MD-scores.

\section{Meta-analyses}

Using PubMed with search terms Mediterranean diet, and breast cancer/neoplasm/tumor, or mammary carcinoma/tumors, cohort studies of the association between MD adherence (a priori defined) and breast cancer were identified up to August 2016. Six articles on breast cancer (ER/PR subtypes) were identified [7, 35-39]. Because Buckland et al. [37] presented EPIC-wide results, the results from specific EPIC countries $[35,39]$ were not included in the meta-analysis to avoid overlap. In addition to EPIC-results, the publication by Pot et al. [39] also included results of Cade et al. [36]. Data on total postmenopausal breast cancer and subtypes of the remaining four cohorts (Nurses' Health Study (NHS), UK Women's Cohort Study (UKWCS), EPIC, and Women's Lifestyle and Health (WLH)) were combined with NLCSdata in the meta-analysis. HRs for the contrast between highest versus lowest category of $\mathrm{MD}$ adherence from each study were pooled using random-effects models. In these analyses, the HR-estimate for each study was weighted by the inverse of the variance of the log HR to calculate the summary HR and its $95 \% \mathrm{Cl}$. Heterogeneity between studies was estimated using the Cochran's $Q$ test and $I^{2}$ (the proportion of variation in HRs attributable to heterogeneity [40]). Publication bias was assessed by the Begg test [41]. Analyses were performed using Stata version 12; presented $p$-values are two-sided, with $p<0.05$ considered as statistically significant. 


\section{Results}

The mean (SD) score of aMEDr among subcohort members was 4.0 (1.6), and for mMEDr 4.0 (1.5). Table 3.1 summarizes several baseline characteristics according to adherence to aMEDr and mMEDr. Conformity with the MD was lower in older women, in nulliparous women, current smokers, and in those with a positive family history of breast cancer (for aMEDr). MD adherence was higher in physically active women, higher educated women, and ever oral contraceptive users. Alcohol intake was somewhat higher in those scoring higher on aMEDr, but this was reversed for mMEDr. Women with a high score on mMEDr more often reported a history of benign breast disease.

Table 3.2 shows results of the age-adjusted and multivariable-adjusted analyses of the associations of MD-scores with total breast cancer risk. While the aMEDr-score was significantly inversely associated with breast cancer risk in age-adjusted analyses, in multivariable-adjusted continuous analyses, the HR per 2-point increment was 0.92 (95\% $\mathrm{Cl}: 0.84,1.01)$. In multivariable-adjusted categorical analyses, only the medium category showed a significantly decreased risk with a $\mathrm{HR}$ of $0.82(95 \% \mathrm{Cl}: 0.70,0.96)$, compared to low adherence scores, and there was no clear decreasing trend across categories ( $p$-trend $=0.066$ ). The AIC of the model using aMEDr was smaller compared to the mMEDr-model, indicating a better fit using aMED-scoring. For comparison, the table also shows associations when using the full aMED and mMED including alcohol. The AIC-values indicated a worse fit for both aMED and MMED when alcohol was included in the scores (Table 3.2). Based on this, ensuing analyses were conducted primarily with aMEDr; at several places we also present results for mMEDr for reasons of comparison.

Table 3.3 shows age- and multivariable-adjusted associations between aMEDr and risk of ER and PR subtypes of breast cancer. There was a stronger inverse association with aMEDr for ER- breast cancer than for ER+ breast cancer, with HRs when comparing high versus low adherence of 0.60 (95\% Cl: $0.39,0.93)$, p-trend=0.032 for ER-, and 0.87 (95\% Cl: $0.69,1.10)$, p-trend $=0.101$ for $E R+$, respectively. The same pattern was seen for PR subtypes, albeit somewhat less strongly inverse in PR- than ER- subtypes. Similarly, ER-PR- breast cancer was significantly inversely related to $M D$ adherence ( $p$-trend $=0.047$ ) with a HR per 2-point increment of $0.75(95 \% \mathrm{Cl}: 0.60,0.94)$, while the ER+PR+ subtype showed no significant association. Heterogeneity tests across subtypes using bootstrapping were not significant. The analyses in Table 3.3 were also conducted with mMEDr. When mMEDr was used, the HRs per 2-point increment were $0.95(95 \% \mathrm{Cl}: 0.85,1.07)$ for $E R+, 0.85(0.71,1.03)$ for ER-, $0.95(0.83,1.08)$ for $P R+, 0.90(0.76,1.07)$ for $P R-, 0.94(0.83,1.08)$ for $E R+P R+$, and 0.79 $(0.63,0.99)$ for ER-PR- breast cancer, i.e., all somewhat weaker associated than with aMEDr.

Estimation of the PAFs indicated that $2.3 \%$ (95\% Cl: $-13.1 \%, 15.5 \%)$ of total breast cancer could be avoided if all participants would shift towards the highest aMEDr category. The estimated PAF for ER+ breast cancer was $2.3 \%$ (95\% Cl: $-16.4 \%, 18.0 \%)$, and $32.4 \%$ (95\% Cl: $4.1 \%, 52.3 \%)$ for ER- breast cancer. If participants would shift their pattern one category upward, the estimated preventable proportions were $4.8 \%$ for total breast cancer, $5.2 \%$ for ER+, and $20.0 \%$ for ER- breast cancer.

Supplementary Table S3.1 shows the HR of breast cancer associated with each of the components of aMEDr, dichotomized at the median intakes, when they were simultaneously entered in the model. Nut intake was significantly inversely associated with ER- breast cancer; other components were mostly weakly inversely associated with breast cancer (subtypes), but not statistically significantly. Table 3.4 shows the HR of breast cancer associated with a 
2-point increment in aMEDr, and how this HR changed after alternate removal of each of its eight components; the percentage reduction in the size of the HR is also presented. For example, when vegetables were excluded from the score, the HR of 0.938 indicated the apparent beneficial effect was reduced by $23.8 \%$, compared to $H R=0.919$ for the full score. These analyses are presented for total breast cancer and ER subtypes. Table 3.4 shows that whole grain intake contributed most to the inverse association for total and ER+ breast cancer, but for ER- breast cancer nut intake seemed most dominant. For total breast cancer, the second and third most dominant components were vegetables and fruit, for ER+ these were vegetables and fish, and for ER- breast cancer these were fruit and the MUFA:SFA ratio. Excluding red and processed meat, and legumes showed opposite effects on HRs for breast cancer, i.e., somewhat stronger HRs.

In Figure 3.1, associations between a 2-point increment in aMEDr and breast cancer are presented, in subgroups of potential effect modifiers: age at baseline, smoking status, alcohol intake, BMI, physical activity, and family history of breast cancer. Inverse associations were seen in most subgroups, and there was no significant interaction. Similarly, associations were essentially similar when the follow-up period was split in 0-2, 2-10, and 10-20 years (Figure 3.1). The corresponding interaction analyses for the ER subtypes of breast cancer are also presented in Figure 3.1. Only for ER- breast cancer, statistically significant interactions were seen with age at baseline and alcohol intake. While aMEDr showed a stronger inverse association with ER- breast cancer in women drinking $15 \mathrm{~g} /$ day of alcohol or more, the inverse association was also more apparent in younger women.

To enable comparison of HR-estimates using aMED-scores with models using the WCRF/ AICR-score for dietary recommendations, the scores were assessed as continuous variables with 1 SD as increment. This was done for the scores including and excluding alcohol. For comparability, models for aMED-scores were rerun with the same participants as in the WCRF/AICR-score models because the inclusion of salt data introduced some additional missing values. The results in Table 3.5 show that the model performance was better (as judged by lower AIC) when using the aMED-scores (excluding or including alcohol), compared to the dietary WCRF/AICR-scores, for total breast cancer and the ER subtypes. The analyses in Table 3.5 were also conducted with mMED. For comparison, when mMED (including alcohol) was used, the HR per 1-SD increment was $0.96(95 \% \mathrm{Cl}: 0.90,1.03)$. When mMEDr was used, the HRs per 1-SD increment were 0.95 (95\% Cl: $0.88,1.02)$ in total breast cancer, $0.95(0.88,1.04)$ in $E R+$, and $0.89(0.77,1.02)$ in ER- breast cancer, i.e., all somewhat weaker associated than with aMEDr. 


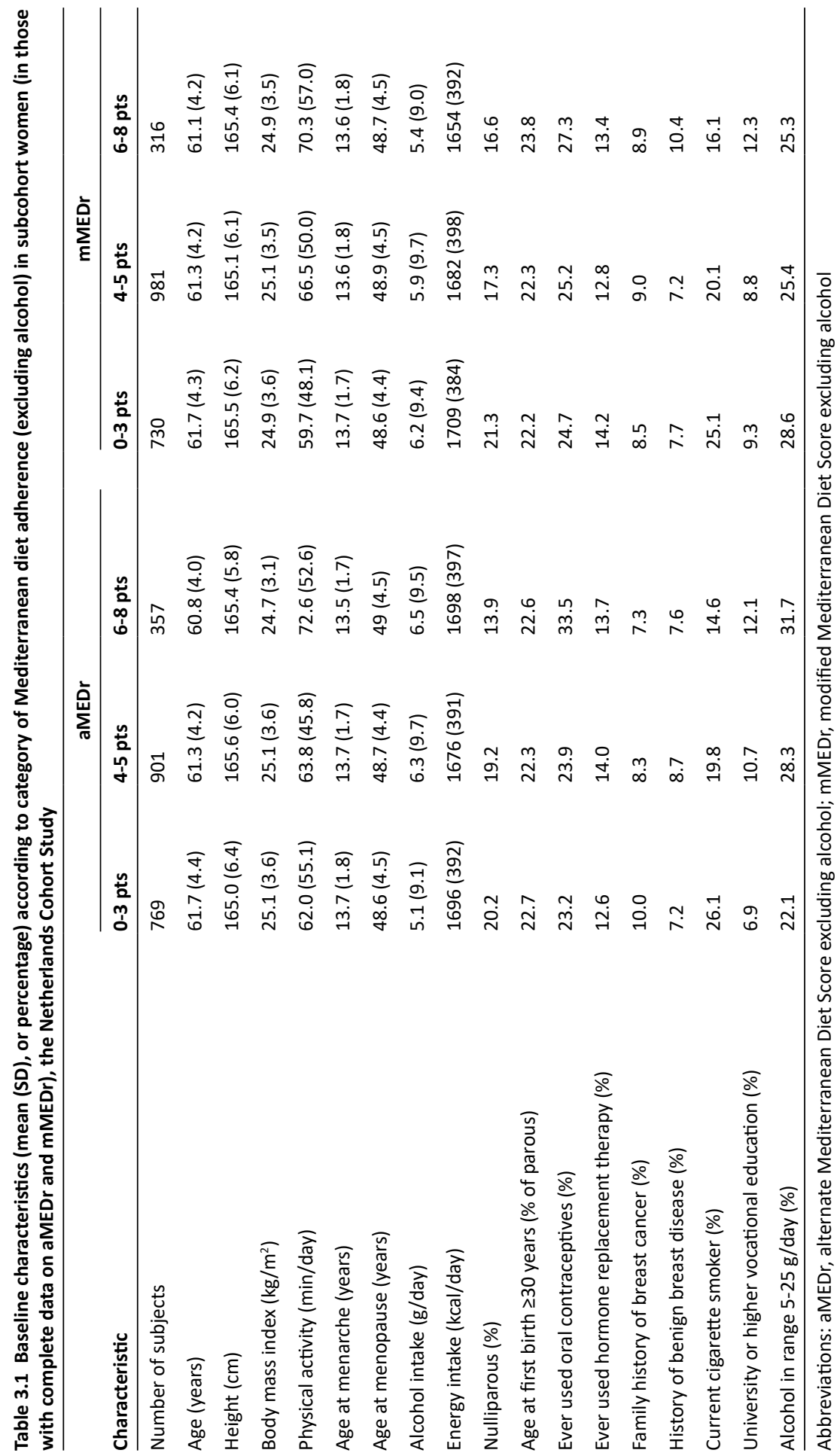




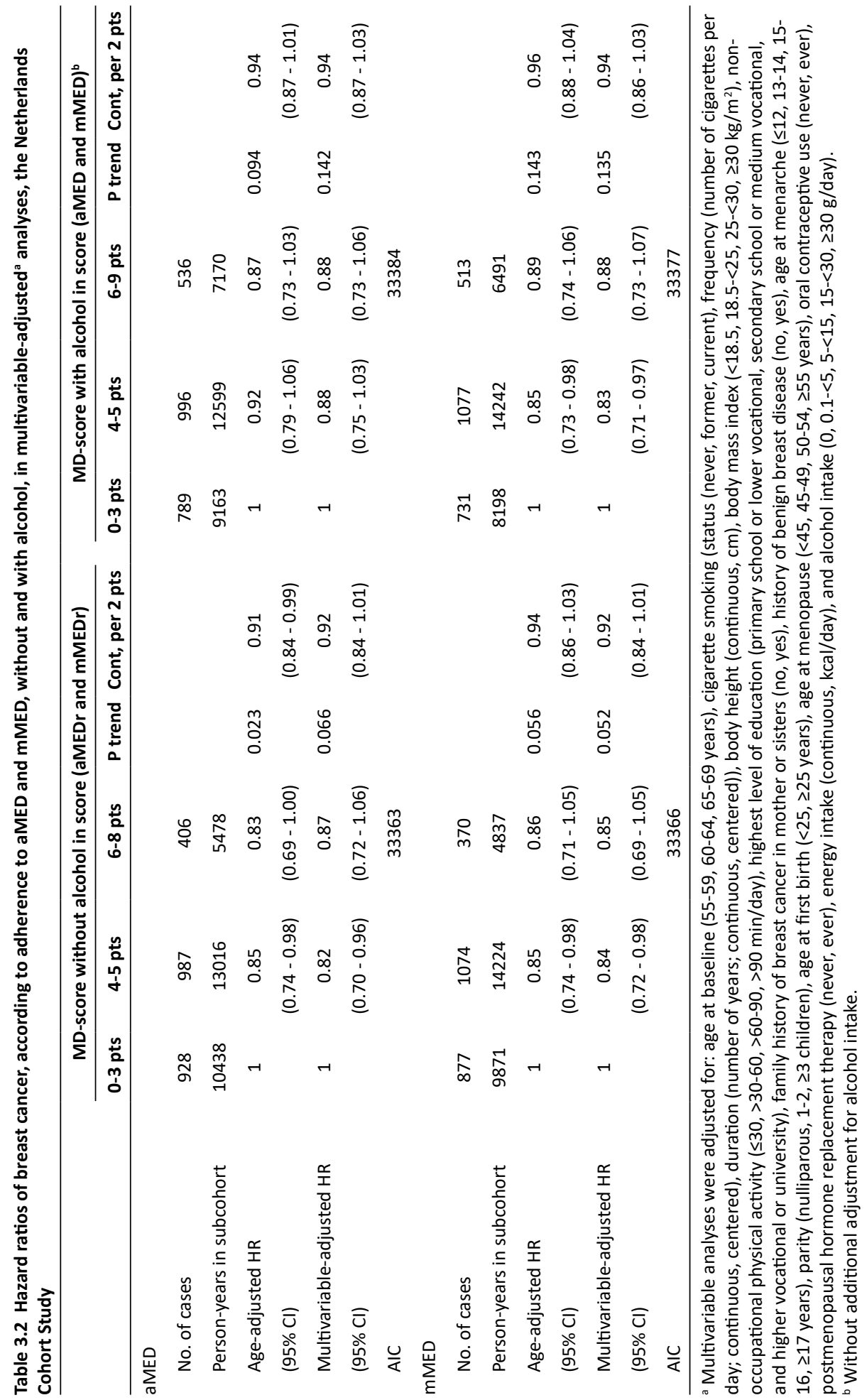


Table 3.3 Hazard ratios of breast cancer subtypes, according to adherence to Mediterranean diet (aMEDr) in multivariable-adjusted ${ }^{a}$ analyses, the Netherlands Cohort Study

\begin{tabular}{|c|c|c|c|c|c|c|}
\hline & \multicolumn{6}{|c|}{ aMEDr } \\
\hline & $0-3$ pts & 4-5 pts & 6-8 pts & $P$ trend & AIC & Cont, per 2 pts \\
\hline \multicolumn{7}{|l|}{ Total breast cancer } \\
\hline No. of cases & 928 & 987 & 406 & & & \\
\hline Person-years in subcohort & 10438 & 13016 & 5478 & & & \\
\hline Age-adjusted HR & 1 & 0.85 & 0.83 & 0.023 & & 0.91 \\
\hline$(95 \% \mathrm{Cl})$ & & $(0.74-0.98)$ & $(0.69-1.00)$ & & & $(0.84-0.99)$ \\
\hline Multivariable-adjusted HR & 1 & 0.82 & 0.87 & 0.066 & 33363 & 0.92 \\
\hline$(95 \% \mathrm{Cl})$ & & $(0.70-0.96)$ & $(0.72-1.06)$ & & & $(0.84-1.01)$ \\
\hline \multicolumn{7}{|l|}{ ER+ breast cancer } \\
\hline No. of cases & 460 & 466 & 195 & & & \\
\hline Age-adjusted HR & 1 & 0.82 & 0.80 & 0.022 & & 0.89 \\
\hline$(95 \% \mathrm{Cl})$ & & $(0.69-0.97)$ & $(0.64-1.00)$ & & & $(0.81-0.99)$ \\
\hline Multivariable-adjusted HR & 1 & 0.81 & 0.87 & 0.101 & 16119 & 0.91 \\
\hline$(95 \% \mathrm{Cl})$ & & $(0.68-0.97)$ & $(0.69-1.10)$ & & & $(0.82-1.02)$ \\
\hline \multicolumn{7}{|l|}{ ER- breast cancer } \\
\hline No. of cases & 100 & 116 & 32 & & & \\
\hline Age-adjusted HR & 1 & 0.93 & 0.59 & 0.024 & & 0.81 \\
\hline$(95 \% \mathrm{Cl})$ & & $(0.69-1.24)$ & $(0.39-0.91)$ & & & $(0.69-0.97)$ \\
\hline Multivariable-adjusted HR & 1 & 0.92 & 0.60 & 0.032 & 3623 & 0.81 \\
\hline$(95 \% \mathrm{Cl})$ & & $(0.67-1.25)$ & $(0.39-0.93)$ & & & $(0.67-0.96)$ \\
\hline \multicolumn{7}{|l|}{ PR+ breast cancer } \\
\hline No. of cases & 276 & 305 & 122 & & & \\
\hline Age-adjusted HR & 1 & 0.89 & 0.83 & 0.139 & & 0.93 \\
\hline$(95 \% \mathrm{Cl})$ & & $(0.73-1.09)$ & $(0.64-1.08)$ & & & $(0.82-1.04)$ \\
\hline Multivariable-adjusted HR & 1 & 0.90 & 0.90 & 0.378 & 10101 & 0.94 \\
\hline$(95 \% \mathrm{Cl})$ & & $(0.73-1.11)$ & $(0.69-1.19)$ & & & $(0.83-1.07)$ \\
\hline \multicolumn{7}{|l|}{ PR- breast cancer } \\
\hline No. of cases & 158 & 157 & 60 & & & \\
\hline Age-adjusted HR & 1 & 0.79 & 0.69 & 0.017 & & 0.80 \\
\hline$(95 \% \mathrm{Cl})$ & & $(0.61-1.01)$ & $(0.48-0.96)$ & & & $(0.69-0.93)$ \\
\hline Multivariable-adjusted HR & 1 & 0.76 & 0.72 & 0.047 & 5422 & 0.81 \\
\hline$(95 \% \mathrm{Cl})$ & & $(0.59-1.00)$ & $(0.52-1.05)$ & & & $(0.69-0.96)$ \\
\hline
\end{tabular}




\section{Chapter 3}

Table 3.3 (continued)

\begin{tabular}{|c|c|c|c|c|c|c|}
\hline & \multicolumn{6}{|c|}{ aMEDr } \\
\hline & $0-3$ pts & 4-5 pts & 6-8 pts & $P$ trend & AIC & Cont, per 2 pts \\
\hline \multicolumn{7}{|l|}{$\mathrm{ER}+\mathrm{PR}+$ breast cancer } \\
\hline No. of cases & 270 & 295 & 120 & & & \\
\hline $\begin{array}{l}\text { Age-adjusted HR } \\
(95 \% \mathrm{Cl})\end{array}$ & 1 & $\begin{array}{c}0.88 \\
(0.72-1.08)\end{array}$ & $\begin{array}{c}0.84 \\
(0.65-1.09)\end{array}$ & 0.146 & & $\begin{array}{c}0.93 \\
(0.83-1.04)\end{array}$ \\
\hline $\begin{array}{l}\text { Multivariable-adjusted HR } \\
(95 \% \mathrm{Cl})\end{array}$ & 1 & $\begin{array}{c}0.89 \\
(0.71-1.10)\end{array}$ & $\begin{array}{c}0.91 \\
(0.69-1.21)\end{array}$ & 0.400 & 9838 & $\begin{array}{c}0.95 \\
(0.83-1.08)\end{array}$ \\
\hline \multicolumn{7}{|l|}{ ER-PR- breast cancer } \\
\hline No. of cases & 71 & 75 & 24 & & & \\
\hline $\begin{array}{l}\text { Age-adjusted HR } \\
(95 \% \mathrm{Cl})\end{array}$ & 1 & $\begin{array}{c}0.83 \\
(0.59-1.18)\end{array}$ & $\begin{array}{c}0.61 \\
(0.37-0.99)\end{array}$ & 0.042 & & $\begin{array}{c}0.77 \\
(0.63-0.95)\end{array}$ \\
\hline $\begin{array}{l}\text { Multivariable-adjusted HR } \\
(95 \% \mathrm{Cl})\end{array}$ & 1 & $\begin{array}{c}0.79 \\
(0.55-1.14)\end{array}$ & $\begin{array}{c}0.61 \\
(0.36-1.01)\end{array}$ & 0.047 & 2483 & $\begin{array}{c}0.75 \\
(0.60-0.94)\end{array}$ \\
\hline
\end{tabular}

${ }^{a}$ Multivariable analyses were adjusted for: age at baseline (55-59, 60-64, 65-69 years), cigarette smoking (status (never, former, current), frequency (number of cigarettes per day; continuous, centered), duration (number of years; continuous, centered)), body height (continuous, cm), body mass index $(<18.5,18.5-<25,25-<30, \geq 30 \mathrm{~kg} /$ $\left.\mathrm{m}^{2}\right)$, non-occupational physical activity $(\leq 30,>30-60,>60-90,>90 \mathrm{~min} /$ day), highest level of education (primary school or lower vocational, secondary school or medium vocational, and higher vocational or university), family history of breast cancer in mother or sisters (no, yes), history of benign breast disease (no, yes), age at menarche $(\leq 12,13-14,15-16, \geq 17$ years), parity (nulliparous, $1-2, \geq 3$ children), age at first birth $(<25, \geq 25$ years), age at menopause ( $<45,45-49,50-54, \geq 55$ years), oral contraceptive use (never, ever), postmenopausal hormone replacement therapy (never, ever), energy intake (continuous, kcal/day), and alcohol intake $(0,0.1-<5$, $5-<15,15-<30, \geq 30$ g/day). 


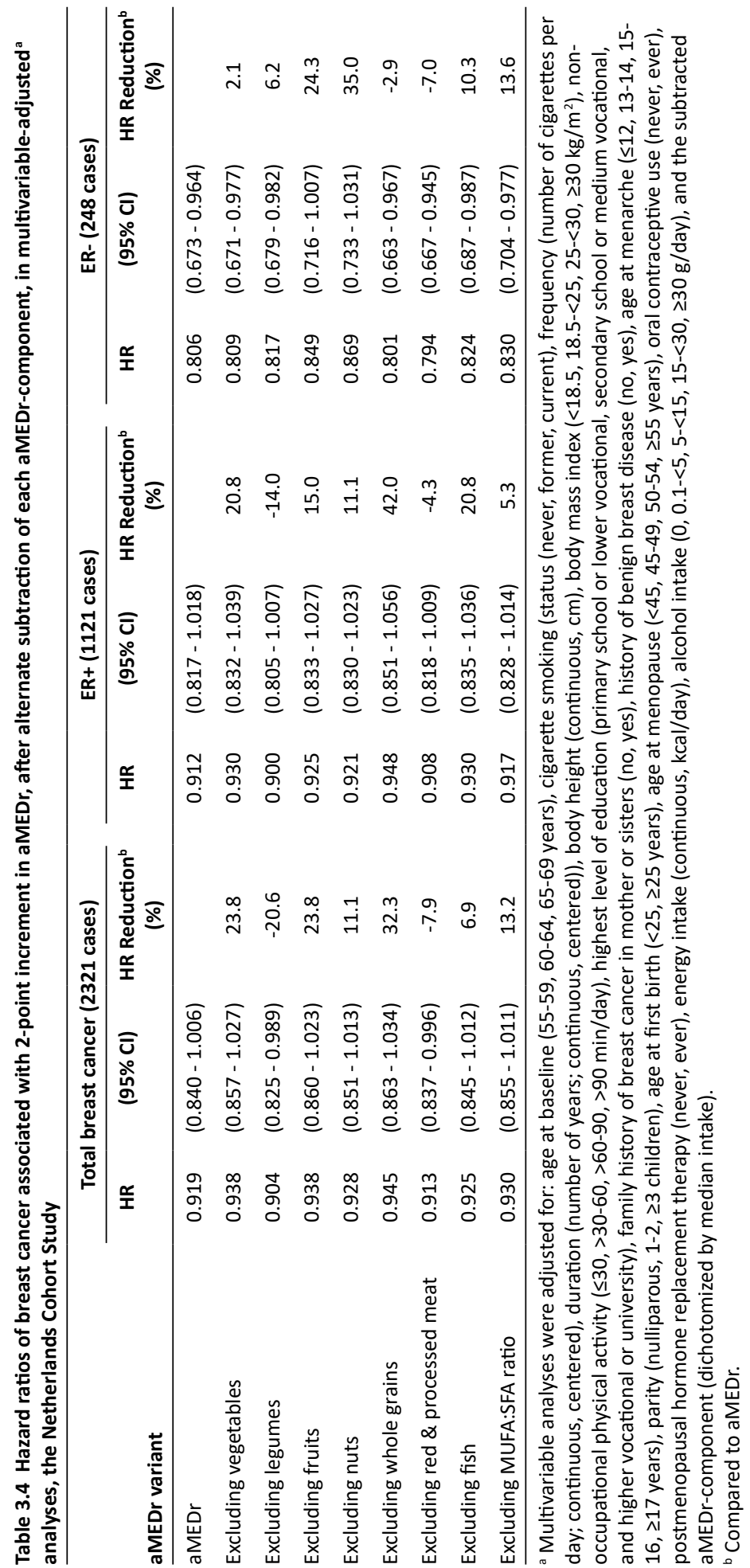




\section{Chapter 3}

Table 3.5 Hazard ratios of breast cancer associated with 1-SD increment in WCRF-diet score compared to aMED, including or excluding alcohol, in multivariable-adjusted ${ }^{a}$ analyses, the Netherlands Cohort Study

\begin{tabular}{|c|c|c|c|}
\hline Score & HR & $(95 \% \mathrm{Cl})$ & AIC \\
\hline \multicolumn{4}{|c|}{ Scores including alcohol } \\
\hline \multicolumn{4}{|c|}{ Breast cancer total (2289 cases) } \\
\hline WCRFdietalc $c^{\mathrm{bc}}$ & 1.01 & $(0.93-1.08)$ & 32853 \\
\hline aMED ${ }^{b}$ & 0.95 & $(0.89-1.02)$ & 32848 \\
\hline \multicolumn{4}{|c|}{ Scores excluding alcohol } \\
\hline \multicolumn{4}{|c|}{ Breast cancer total (2289 cases) } \\
\hline WCRFdiet $^{\mathrm{d}}$ & 1.02 & $(0.94-1.09)$ & 32844 \\
\hline aMEDr & 0.94 & $(0.87-1.01)$ & 32837 \\
\hline \multicolumn{4}{|c|}{ Breast cancer ER+ (1108 cases) } \\
\hline WCRFdiet $^{\mathrm{d}}$ & 0.99 & $(0.90-1.08)$ & 15826 \\
\hline aMEDr & 0.92 & $(0.85-1.01)$ & 15820 \\
\hline \multicolumn{4}{|c|}{ Breast cancer ER- (244 cases) } \\
\hline WCRFdiet $^{\mathrm{d}}$ & 1.00 & $(0.86-1.16)$ & 3532 \\
\hline aMEDr & 0.84 & $(0.73-0.97)$ & 3525 \\
\hline
\end{tabular}

${ }^{a}$ Multivariable analyses were adjusted for: age at baseline (55-59, 60-64, 65-69 years), cigarette smoking (status (never, former, current), frequency (number of cigarettes per day; continuous, centered), duration (number of years; continuous, centered)), body height (continuous, $\mathrm{cm})$, body mass index $(<18.5,18.5$ $\left.<25,25-<30, \geq 30 \mathrm{~kg} / \mathrm{m}^{2}\right)$, non-occupational physical activity $(\leq 30,>30-60,>60-90,>90 \mathrm{~min} /$ day), highest level of education (primary school or lower vocational, secondary school or medium vocational, and higher vocational or university), family history of breast cancer in mother or sisters (no, yes), history of benign breast disease (no, yes), age at menarche ( $\leq 12,13-14,15-16, \geq 17$ years), parity (nulliparous, 1-2, $\geq 3$ children), age at first birth ( $<25, \geq 25$ years), age at menopause $(<45,45-49,50-54, \geq 55$ years), oral contraceptive use (never, ever), postmenopausal hormone replacement therapy (never, ever), energy intake (continuous, $\mathrm{kcal} / \mathrm{day}$ ), and alcohol intake (0, $0.1-<5,5-<15,15-<30, \geq 30 \mathrm{~g} / \mathrm{day})$.

${ }^{\mathrm{b}}$ Model excluding alcohol as covariate.

c WCRF/AICR dietary recommendations including alcohol.

${ }^{\mathrm{d}}$ WCRF/AICR dietary recommendations excluding alcohol. 


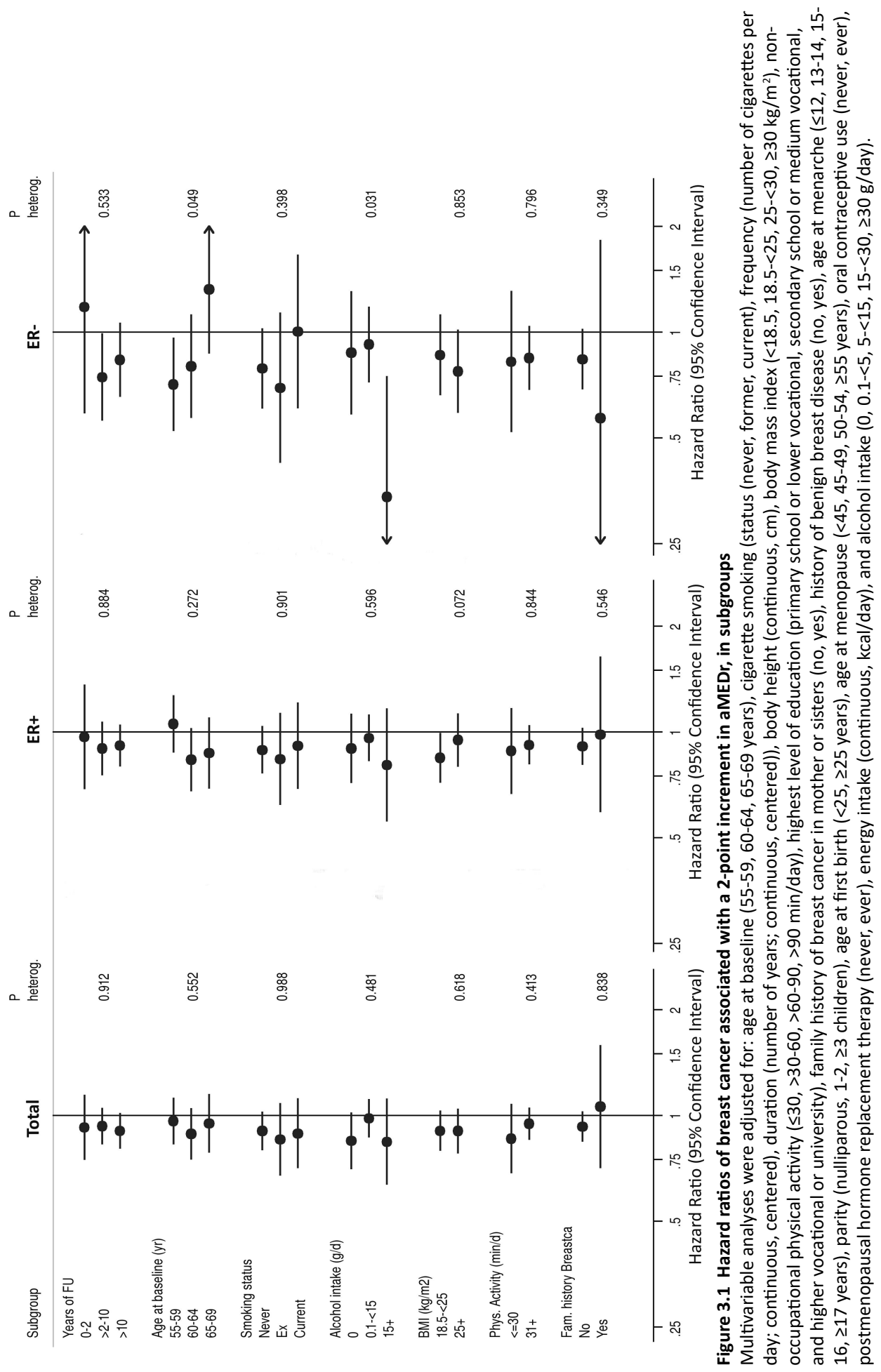




\section{Meta-analyses}

The forest plots and summary estimates for highest versus lowest MD adherence category are presented in Figure 3.2, for total postmenopausal breast cancer and subtypes, when at least two studies were available. For total breast cancer, the summary $\mathrm{HR}(95 \% \mathrm{Cl})$ was $0.94(0.88,1.01)$, with no evidence of between-study heterogeneity $(p=0.330)$. While there was no evidence for an association with $\mathrm{ER}+$ breast cancer, the common $\mathrm{HRs}(95 \% \mathrm{Cl})$ for ER- and ER-PR- breast cancer (each based on two cohorts) were $0.73(0.57,0.93)$ and 0.77 $(0.63,0.94)$, respectively, with no evidence of between-study heterogeneity. As a further sensitivity analysis, Supplementary Figure S3.2 shows results of meta-analyses of studies on total postmenopausal breast cancer that included or excluded alcohol from the MD-score, respectively. When alcohol was excluded, the summary $\mathrm{HR}(95 \% \mathrm{Cl})$ was $0.92(0.87,0.98)$, while there was no association when alcohol was included.

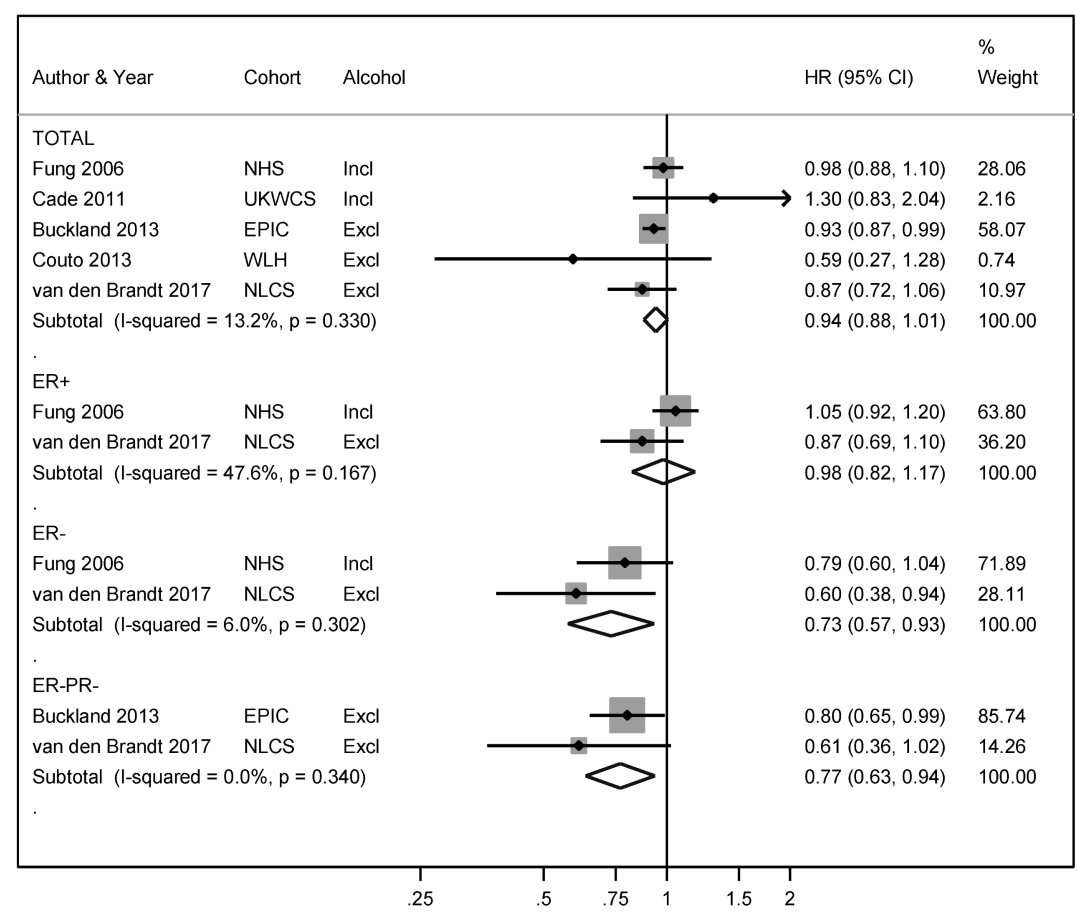

Figure 3.2 Forest plots of postmenopausal breast cancer hazard ratios (HRs) and 95\% confidence intervals (95\% $\mathrm{Cls}$ ) comparing highest versus lowest category of adherence to $\mathrm{MD}$, from random-effects meta-analyses Separate plots are presented for total postmenopausal breast cancer and subtypes. Studies are referred to by first author, year of publication, and cohort abbreviation (EPIC: European Prospective Investigation into Cancer and Nutrition; NHS: Nurses' Health Study; NLCS: Netherlands Cohort Study; UKWCS: UK Women's Cohort Study; WLH: Women's Lifestyle and Health). In addition, it is indicated whether or not alcohol was included in the MD-score. Studies are weighted according to the inverse of the variance of the log HR estimate. The HRs are represented by the squares (the size is proportional to the weights used in the meta-analysis) and $95 \% \mathrm{Cls}$ are represented by the error bars. Diamonds represent the summary HR estimates and $95 \% \mathrm{Cls}$ per endpoint. 


\section{Discussion}

In this large prospective study, we found a statistically significant inverse association between adherence to MD and risk of ER-postmenopausal breast cancer, with a HR of 0.60 for high versus low adherence to $\mathrm{MD}$. There were no significant inverse associations with $\mathrm{ER}+$ or total breast cancer risk. The model fit was better when alcohol was excluded from the aMED-score, and the aMED performed better than the MMED in our cohort. We found no association between breast cancer and adherence to WCRF/AICR-dietary recommendations. In meta-analyses, summary HRs for high versus low $M D$ adherence were 0.94 for total postmenopausal breast cancer, 0.98 for ER+, 0.73 for ER-, and 0.77 for ER-PR- breast cancer. When alcohol was excluded from MD-scores, the summary HR $(95 \% \mathrm{Cl})$ was $0.92(0.87,0.98)$ for total breast cancer, while there was no association when alcohol was included.

Several cohort studies have investigated the association between an a priori defined MDpattern and overall breast cancer risk, or subtypes. In the NHS-cohort, no association was found with total or ER+ postmenopausal breast cancer risk [7], but for ER- breast cancer, the HR comparing highest to lowest quintiles of aMED (including alcohol) was 0.79 ( $p$-trend= 0.03). In EPIC-overall, high versus low rMED-score (variant of mMED) (excluding alcohol) was related to reduced postmenopausal breast cancer risk ( $\mathrm{HR}=0.93)$, especially with $\mathrm{ER}-/$ PR- tumors ( $H R=0.80)$, and not with premenopausal breast cancer [37]. Thus, our findings are in accordance with these cohorts. In a Swedish cohort study, high versus low mMEDscore (including alcohol) was non-significantly inversely associated with postmenopausal breast cancer ( $\mathrm{HR}=0.59)$, but no association was observed in continuous analyses [38]. Apart from overall EPIC-results, there are also some country-specific reports. MD adherence was inversely associated with overall postmenopausal breast cancer in EPIC-Greece (HR=0.78 per 2-point increment) [35]. A UK Cohort consortium which included EPIC-Oxford and EPICNorfolk reported no association between MD adherence and breast cancer [39], but no information was available on ER/PR status. In EPIC-France, an inverse association was found between an a posteriori defined "healthy/Mediterranean" diet and postmenopausal breast cancer, particularly in ER+/PR- tumors, but not in ER-tumors [42].

Our meta-analysis of cohort studies did not show a significant inverse association between overall postmenopausal breast cancer and high versus low MD adherence, although the HR-estimate of 0.94 was marginally significant, with no obvious heterogeneity. However, in contrast to ER+, our meta-analysis showed inverse associations with ER- or ER-PR- breast cancer subtypes, with significant HRs of 0.73 and 0.77 , respectively. Although still based on few cohort studies, these subtype findings may be of particular importance because identification of preventive factors for ER- breast cancers may help to reduce the burden of breast cancer since these tumors respond less well to treatment and have lower five-year survival rates than ER+ tumors. As has been suggested before [7, 37], any potential influence of dietary factors may be difficult to detect in ER+ tumors given the strong influence of hormonal factors. In ER- tumors, other risk factors, including diet, may exert a relatively larger influence and be more easily detectable [7].

Interestingly, a recent secondary analysis of a RCT on primary prevention of cardiovascular diseases (PREDIMED) indicated a strong protective effect of MD versus low-fat diet on the risk of postmenopausal breast cancer in Spain, with a $\mathrm{HR}$ of 0.43 (95\% Cl: $0.21,0.88$ ). The effect was stronger in those randomized to the MD supplemented with extra-virgin olive oil than with nuts, but in both MD-intervention groups the effect was significant [6]. Nevertheless, because the trial had only 35 incident breast cancer cases as outcome, this 
needs to be confirmed in larger trials, preferably also with analyses per receptor subtype.

Consistent with evidence on alcohol and breast cancer [8], our model fit was also worse when moderate alcohol was included in the scores. This was also confirmed in our metaanalysis of MD-scores excluding and including alcohol. In our study, the performance of models with aMED-scores was better than with mMED-scores. This may possibly be due to the fact that the cereal group in MMED aggregates refined and whole grain cereals, while aMED uses whole grain cereals; both cereal types may have distinct effects on breast cancer risk $[37,43]$. In our analysis with aMED-components, whole grain intake contributed most to the inverse association for total and ER+ breast cancer, whereas nut intake seemed most dominant for associations with ER- breast cancer. Such an analysis of dominant components has only been done before with total mortality using mMED [30], which makes it difficult to compare with our results. We did not specifically use a MD-score that included olive oil as component. Olive oil was infrequently used in the NLCS population in 1986, as in many non-Mediterranean countries. Therefore, the MMED was developed [20] in which fatty acid intake is assessed by calculating the ratio of unsaturated (MUFA + PUFA) fatty acids to SFA, to allow for the low consumption of olive oil-derived MUFA in non-Mediterranean countries.

The potential beneficial effects of the MD on cancer risk have been attributed to high amounts of fiber, antioxidants including polyphenols, and vitamins, and may be mediated through several biological mechanisms such as chronic inflammation and oxidative stress [44], and associated DNA oxidative damage [45], and through body weight regulation [46]. The evidence of the cancer protective effect of the MD-pattern is generally stronger than the evidence for individual foods, food groups, or nutrients and cancer risk [1]. Some possible explanations of this could be that interactions and synergisms exist between the components; individual components could also have health effects that are undetectable alone but when integrated with other foods or nutrients in a dietary index, the health benefits become more pronounced [19]. In addition, dietary indexes can overcome the issues of collinearity or confounding between components in the score, and dietary pattern indexes evaluate only the extremes of cumulative exposure, limiting the background noise of individual components [47].

According to a recent review [48], six cohort studies [31, 49-53] investigated the association between adherence to WCRF/AICR-cancer prevention guidelines and breast cancer incidence. These guidelines contain a dietary part (including alcohol) and a nondietary part (body fatness, physical activity, breastfeeding). Most, but not all [52], studies found a lower breast cancer risk for high versus low adherence to these guidelines. We compared the performance of the dietary part of these guidelines with MD adherence (+/- alcohol), and found that models with the MD-score performed better in our population. We found no association between breast cancer and adherence to WCRF/AICR-dietary recommendations. This might seem in contrast with the inverse associations in the earlier cohort studies, but these primarily investigated dietary and nondietary recommendations combined (i.e., including overweight and physical activity (and lactation)). It might be that these nondietary factors were dominating the inverse associations reported earlier. For example, Nomura et al. [51] found no effect of dietary recommendations beyond BMI and alcohol, but such dominance was not reported by Catsburg et al. [53]. When comparing our results with those of Romaguera et al. [31], whose operationalization of the dietary guidelines we followed (except salt), they reported a HR of 0.95 per 1-point increment in their 7-component score. However, their score also included nondietary recommendations; further research on this is needed. A recent pooled analysis of seven cohort studies also showed no association 
between WCRF/AICR-dietary recommendations and breast cancer risk [54].

The prospective design and high completeness of follow-up of the NLCS make information bias and selection bias unlikely. A potential weakness is the moderate proportion of breast cancer cases for whom ER/PR status was known. Breast cancer cases with known and unknown receptor status did not differ importantly according to baseline and tumor characteristics, making selection bias of the cases unlikely (data not shown). Although many possible confounders were taken into account, the possibility of confounding by unmeasured factors remains. The validation study of the food frequency questionnaire has shown that it performs relatively well [13], but measurement error may still have attenuated associations. The lack of possibilities to update dietary intake or other lifestyle data during follow-up may have resulted in some attenuated associations too.

In conclusion, our cohort study showed, in accordance with major cohort studies as the NHS and EPIC, that MD adherence showed moderately strong inverse associations with risk of ER( $40 \%$ reduction), and ER-PR- (39\% reduction) breast cancers, and weak inverse associations with ER+ and total postmenopausal breast cancer. Assuming causality, we estimated that $32.4 \%$ of ER- breast cancer, and $2.3 \%$ of total and ER+ breast cancer could be avoided if the population would shift intake towards the highest MD category. 


\section{References}

1. World Cancer Research Fund/American Institute for Cancer Research. Food, nutrition, physical activity, and the prevention of cancer: a global perspective. Washington DC: AICR; 2007.

2. Hu FB. Dietary pattern analysis: a new direction in nutritional epidemiology. Curr Opin Lipidol. 2002;13(1):3-9.

3. Fung TT, Rexrode KM, Mantzoros CS, Manson JE, Willett WC, Hu FB. Mediterranean diet and incidence of and mortality from coronary heart disease and stroke in women. Circulation. 2009;119(8):1093-100.

4. Willett WC, Sacks F, Trichopoulou A, Drescher G, Ferro-Luzzi A, Helsing E, et al. Mediterranean diet pyramid: a cultural model for healthy eating. Am J Clin Nutr. 1995;61(6 Suppl):1402S-6S.

5. Schwingshackl L, Hoffmann G. Adherence to Mediterranean diet and risk of cancer: an updated systematic review and meta-analysis of observational studies. Cancer Med. 2015;4(12):193347.

6. Toledo E, Salas-Salvado J, Donat-Vargas C, Buil-Cosiales P, Estruch R, Ros E, et al. Mediterranean Diet and Invasive Breast Cancer Risk Among Women at High Cardiovascular Risk in the PREDIMED Trial: A Randomized Clinical Trial. JAMA Intern Med. 2015;175(11):1752-60.

7. Fung TT, Hu FB, McCullough ML, Newby PK, Willett WC, Holmes MD. Diet quality is associated with the risk of estrogen receptor-negative breast cancer in postmenopausal women. J Nutr. 2006;136(2):466-72.

8. Smith-Warner SA, Spiegelman D, Yaun SS, van den Brandt PA, Folsom AR, Goldbohm RA, et al. Alcohol and breast cancer in women: a pooled analysis of cohort studies. JAMA. 1998;279(7):53540.

9. van den Brandt PA, Goldbohm RA, van 't Veer P, Volovics A, Hermus RJ, Sturmans F. A largescale prospective cohort study on diet and cancer in The Netherlands. J Clin Epidemiol. 1990;43(3):28595.

10. Prentice RL. A case-cohort design for epidemiologic studies and disease prevention trials. Biometrika. 1986;73(1):1-11.

11. van den Brandt PA, Schouten LJ, Goldbohm RA, Dorant E, Hunen PM. Development of a record linkage protocol for use in the Dutch Cancer Registry for Epidemiological Research. Int J Epidemiol. 1990;19(3):553-8.

12. van den Brandt PA, van 't Veer P, Goldbohm RA, Dorant E, Volovics A, Hermus RJ, et al. A prospective cohort study on dietary fat and the risk of postmenopausal breast cancer. Cancer Res. 1993;53(1):75-82.

13. Goldbohm RA, van den Brandt PA, Brants HA, van 't Veer P, Al M, Sturmans F, et al. Validation of a dietary questionnaire used in a large-scale prospective cohort study on diet and cancer. Eur J Clin Nutr. 1994;48(4):253-65.

14. Nevo-Table. Dutch food composition table 1986-1987; Nederlands voedingsstoffenbestand 1986-1987. The Hague, the Netherlands: Voorlichtingsbureau voor de Voeding; 1986.

15. Dirx MJ, Voorrips LE, Goldbohm RA, van den Brandt PA. Baseline recreational physical activity, history of sports participation, and postmenopausal breast carcinoma risk in the Netherlands Cohort Study. Cancer. 2001;92(6):1638-49.

16. Fung TT, McCullough ML, Newby PK, Manson JE, Meigs JB, Rifai N, et al. Diet-quality scores and plasma concentrations of markers of inflammation and endothelial dysfunction. Am J Clin Nutr. 2005;82(1):163-73.

17. Mitrou PN, Kipnis V, Thiebaut AC, Reedy J, Subar AF, Wirfalt E, et al. Mediterranean dietary pattern and prediction of all-cause mortality in a US population: results from the NIH-AARP Diet and Health Study. Arch Intern Med. 2007;167(22):2461-8.

18. Trichopoulou A, Kouris-Blazos A, Wahlqvist ML, Gnardellis C, Lagiou P, Polychronopoulos E, et al. Diet and overall survival in elderly people. BMJ. 1995;311(7018):1457-60.

19. Trichopoulou A, Costacou T, Bamia C, Trichopoulos D. Adherence to a Mediterranean diet and survival in a Greek population. N Engl J Med. 2003;348(26):2599-608.

20. Trichopoulou A, Orfanos P, Norat T, Bueno-de-Mesquita B, Ocke MC, Peeters PH, et al. Modified Mediterranean diet and survival: EPIC-elderly prospective cohort study. BMJ. 2005;330(7498):991.

21. Schoenfeld D. Partial residuals for the proportional hazards regression model. Biometrika. 
1982;69:239-41.

22. Lin D, Wei L. The robust inference for the Cox proportional hazards model. J Am Stat Assoc. 1989;84(408):1074-8.

23. Akaike H. A new look at the statistical model identification. IEEE Transactions and Automatic Control. 1974;AC-19:716-23.

24. de Vogel S, Bongaerts BW, Wouters KA, Kester AD, Schouten LJ, de Goeij AF, et al. Associations of dietary methyl donor intake with MLH1 promoter hypermethylation and related molecular phenotypes in sporadic colorectal cancer. Carcinogenesis. 2008;29(9):1765-73.

25. Wacholder S, Gail M, Pee D, Brookmeyer R. Alternative variance and efficiency calculations for the case-cohort design. Biometrika. 1989;76(1):117-23.

26. Rockhill B, Newman B, Weinberg C. Use and misuse of population attributable fractions. Am J Public Health. 1998;88(1):15-9.

27. Wahrendorf J. An estimate of the proportion of colo-rectal and stomach cancers which might be prevented by certain changes in dietary habits. Int J Cancer. 1987;40(5):625-8.

28. Leenders M, Sluijs I, Ros MM, Boshuizen HC, Siersema PD, Ferrari P, et al. Fruit and vegetable consumption and mortality: European prospective investigation into cancer and nutrition. Am J Epidemiol. 2013;178(4):590-602.

29. Newson RB. Attributable and unattributable risks and fractions and other scenario comparisons. Stata J. 2013;13:672-98.

30. Trichopoulou A, Bamia C, Trichopoulos D. Anatomy of health effects of Mediterranean diet: Greek EPIC prospective cohort study. BMJ. 2009;338:b2337.

31. Romaguera D, Vergnaud AC, Peeters PH, van Gils CH, Chan DS, Ferrari P, et al. Is concordance with World Cancer Research Fund/American Institute for Cancer Research guidelines for cancer prevention related to subsequent risk of cancer? Results from the EPIC study. Am J Clin Nutr. 2012;96(1):150-63.

32. Vergnaud AC, Romaguera D, Peeters PH, van Gils CH, Chan DS, Romieu I, et al. Adherence to the World Cancer Research Fund/American Institute for Cancer Research guidelines and risk of death in Europe: results from the European Prospective Investigation into Nutrition and Cancer cohort study. Am J Clin Nutr. 2013;97(5):1107-20.

33. Deckers IA, van den Brandt PA, van Engeland M, Soetekouw PM, Baldewijns MM, Goldbohm RA, et al. Long-term dietary sodium, potassium and fluid intake; exploring potential novel risk factors for renal cell cancer in the Netherlands Cohort Study on diet and cancer. Br J Cancer. 2014;110(3):797801.

34. Kromhout D, Spaaij CJ, de Goede J, Weggemans RM. The 2015 Dutch food-based dietary guidelines. Eur J Clin Nutr. 2016;70(8):869-78.

35. Trichopoulou A, Bamia C, Lagiou P, Trichopoulos D. Conformity to traditional Mediterranean diet and breast cancer risk in the Greek EPIC (European Prospective Investigation into Cancer and Nutrition) cohort. Am J Clin Nutr. 2010;92(3):620-5.

36. Cade JE, Taylor EF, Burley VJ, Greenwood DC. Does the Mediterranean dietary pattern or the Healthy Diet Index influence the risk of breast cancer in a large British cohort of women? Eur J Clin Nutr. 2011;65(8):920-8.

37. Buckland G, Travier N, Cottet V, Gonzalez CA, Lujan-Barroso L, Agudo A, et al. Adherence to the Mediterranean diet and risk of breast cancer in the European Prospective Investigation into Cancer and Nutrition cohort study. Int J Cancer. 2013;132(12):2918-27.

38. Couto E, Sandin S, Lof M, Ursin G, Adami HO, Weiderpass E. Mediterranean dietary pattern and risk of breast cancer. PLoS One. 2013;8(2):e55374.

39. Pot GK, Stephen AM, Dahm CC, Key TJ, Cairns BJ, Burley VJ, et al. Dietary patterns derived with multiple methods from food diaries and breast cancer risk in the UK Dietary Cohort Consortium. Eur J Clin Nutr. 2014;68(12):1353-8.

40. Higgins JP, Thompson SG. Quantifying heterogeneity in a meta-analysis. Stat Med. 2002;21(11):1539-58.

41. Begg CB, Mazumdar M. Operating characteristics of a rank correlation test for publication bias. Biometrics. 1994;50(4):1088-101.

42. Cottet V, Touvier M, Fournier A, Touillaud MS, Lafay L, Clavel-Chapelon F, et al. Postmenopausal breast cancer risk and dietary patterns in the E3N-EPIC prospective cohort study. Am J Epidemiol. 
2009;170(10):1257-67.

43. Aune D, Chan DS, Greenwood DC, Vieira AR, Rosenblatt DA, Vieira R, et al. Dietary fiber and breast cancer risk: a systematic review and meta-analysis of prospective studies. Ann Oncol. 2012;23(6):1394-402.

44. Verberne L, Bach-Faig A, Buckland G, Serra-Majem L. Association between the Mediterranean diet and cancer risk: a review of observational studies. Nutr Cancer. 2010;62(7):860-70.

45. Mitjavila MT, Fandos M, Salas-Salvado J, Covas MI, Borrego S, Estruch R, et al. The Mediterranean diet improves the systemic lipid and DNA oxidative damage in metabolic syndrome individuals. A randomized, controlled, trial. Clin Nutr. 2013;32(2):172-8.

46. Romaguera D, Norat T, Vergnaud AC, Mouw T, May AM, Agudo A, et al. Mediterranean dietary patterns and prospective weight change in participants of the EPIC-PANACEA project. Am J Clin Nutr. 2010;92(4):912-21.

47. Jacques PF, Tucker KL. Are dietary patterns useful for understanding the role of diet in chronic disease? Am J Clin Nutr. 2001;73(1):1-2.

48. Kohler LN, Garcia DO, Harris RB, Oren E, Roe DJ, Jacobs ET. Adherence to Diet and Physical Activity Cancer Prevention Guidelines and Cancer Outcomes: A Systematic Review. Cancer Epidemiol Biomarkers Prev. 2016;25(7):1018-28.

49. Hastert TA, Beresford SA, Patterson RE, Kristal AR, White E. Adherence to WCRF/AICR cancer prevention recommendations and risk of postmenopausal breast cancer. Cancer Epidemiol Biomarkers Prev. 2013;22(9):1498-508.

50. Harris HR, Bergkvist L, Wolk A. Adherence to the World Cancer Research Fund/American Institute for Cancer Research recommendations and breast cancer risk. Int J Cancer. 2016;138(11):265764.

51. Nomura SJ, Dash C, Rosenberg L, Yu J, Palmer JR, Adams-Campbell LL. Adherence to diet, physical activity and body weight recommendations and breast cancer incidence in the Black Women's Health Study. Int J Cancer. 2016;139(12):2738-52.

52. Makarem N, Lin Y, Bandera EV, Jacques PF, Parekh N. Concordance with World Cancer Research Fund/American Institute for Cancer Research (WCRF/AICR) guidelines for cancer prevention and obesity-related cancer risk in the Framingham Offspring cohort (1991-2008). Cancer Causes Control. 2015;26(2):277-86.

53. Catsburg C, Miller AB, Rohan TE. Adherence to cancer prevention guidelines and risk of breast cancer. Int J Cancer. 2014;135(10):2444-52.

54. Jankovic N, Geelen A, Winkels RM, Mwungura B, Fedirko V, Jenab M, et al. Adherence to the WCRF/AICR Dietary Recommendations for Cancer Prevention and Risk of Cancer in Elderly from Europe and the United States: A Meta-Analysis within the CHANCES Project. Cancer Epidemiol Biomarkers Prev. 2017;26(1):136-44. 


\section{Supplementary materials}

\begin{tabular}{|c|c|}
\hline \multicolumn{2}{|c|}{ Netherlands Cohort Study on diet and cancer ( $N=62573$ female participants) } \\
\hline$\downarrow$ & $\downarrow$ \\
\hline Random subcohort & 20.3 yr Cancer incidence follow-up: number of cases \\
\hline$\downarrow$ & $\downarrow$ \\
\hline 2589 & Breast cancer \\
\hline
\end{tabular}

\begin{tabular}{|c|c|c|c|c|c|}
\hline$\downarrow$ & $\downarrow$ & & & & \\
\hline \multirow[t]{5}{*}{2438} & 3354 & & & & \\
\hline & Exclusion of $n$ & nelial a & e inva & & \\
\hline & $\downarrow$ & $\downarrow$ & $\downarrow$ & $\downarrow$ & $\downarrow$ \\
\hline & Breast cancer & ER+ & ER- & $\mathrm{PR}+$ & PR- \\
\hline & 3339 & 1618 & 364 & 1008 & 556 \\
\hline
\end{tabular}

\begin{tabular}{|c|c|c|c|c|c|}
\hline \multicolumn{6}{|c|}{ Exclusion of participants with incomplete or inconsistent dietary data } \\
\hline$\downarrow$ & $\downarrow$ & $\downarrow$ & $\downarrow$ & $\downarrow$ & $\downarrow$ \\
\hline 2248 & 3081 & 1439 & 324 & 900 & 499 \\
\hline
\end{tabular}

\begin{tabular}{|ccccccc|}
\hline Exclusion of participants with inconsistent vegetable data and missing alcohol data & & \\
$\downarrow \downarrow$ & $\downarrow$ & $\downarrow$ & $\downarrow$ & $\downarrow$ & $\downarrow$ & 468 \\
2027 & 2818 & 1381 & 303 & 864 & 468
\end{tabular}

\begin{tabular}{|ccccccc|}
\hline Exclusion of participants with missing values on covariates & & & \\
$\downarrow$ & $\downarrow$ & $\downarrow$ & $\downarrow$ & $\downarrow$ & $\downarrow$ \\
1665 & 2321 & 1121 & 248 & 703 & 375
\end{tabular}

Supplementary Figure S3.1 Flow diagram of the number of subcohort members and breast cancer cases on which analyses are based, the Netherlands Cohort Study 


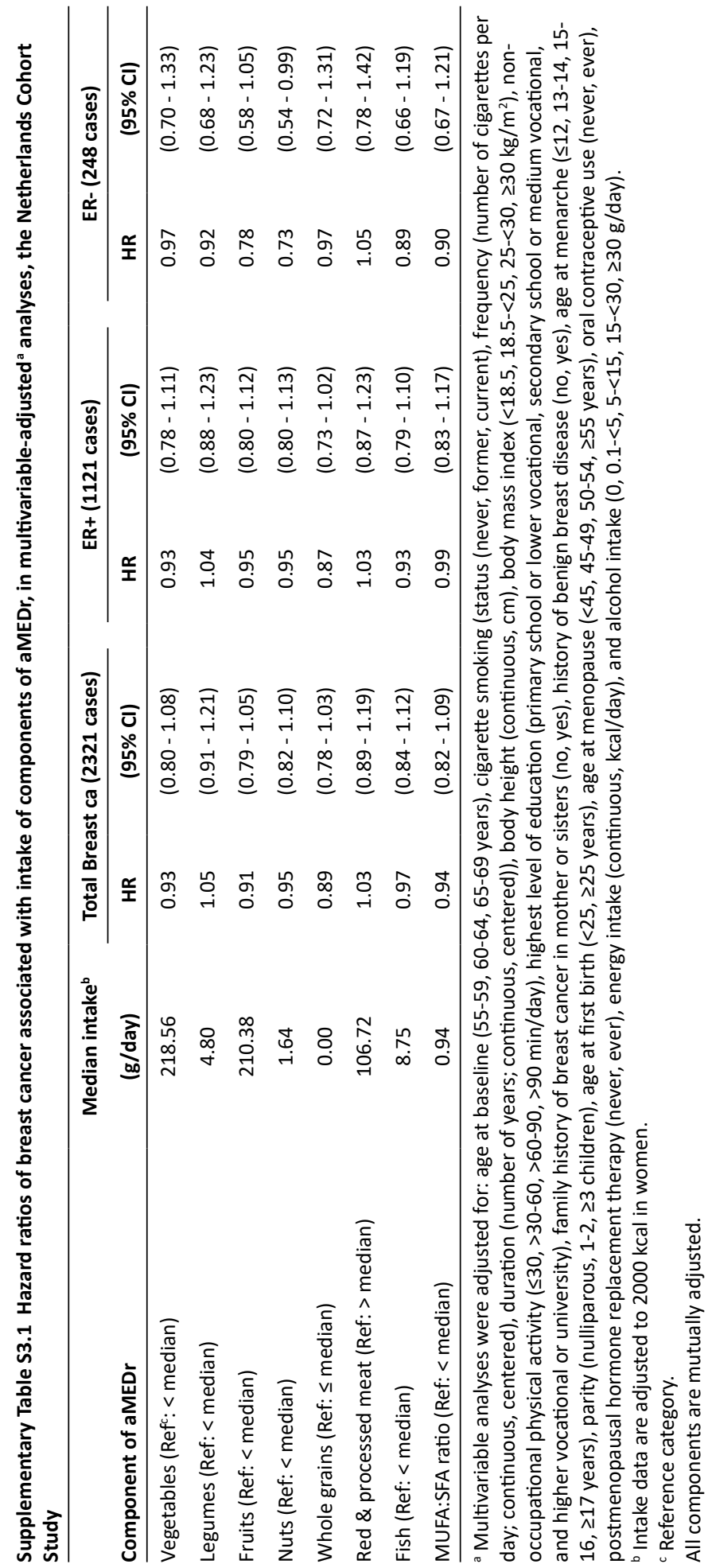




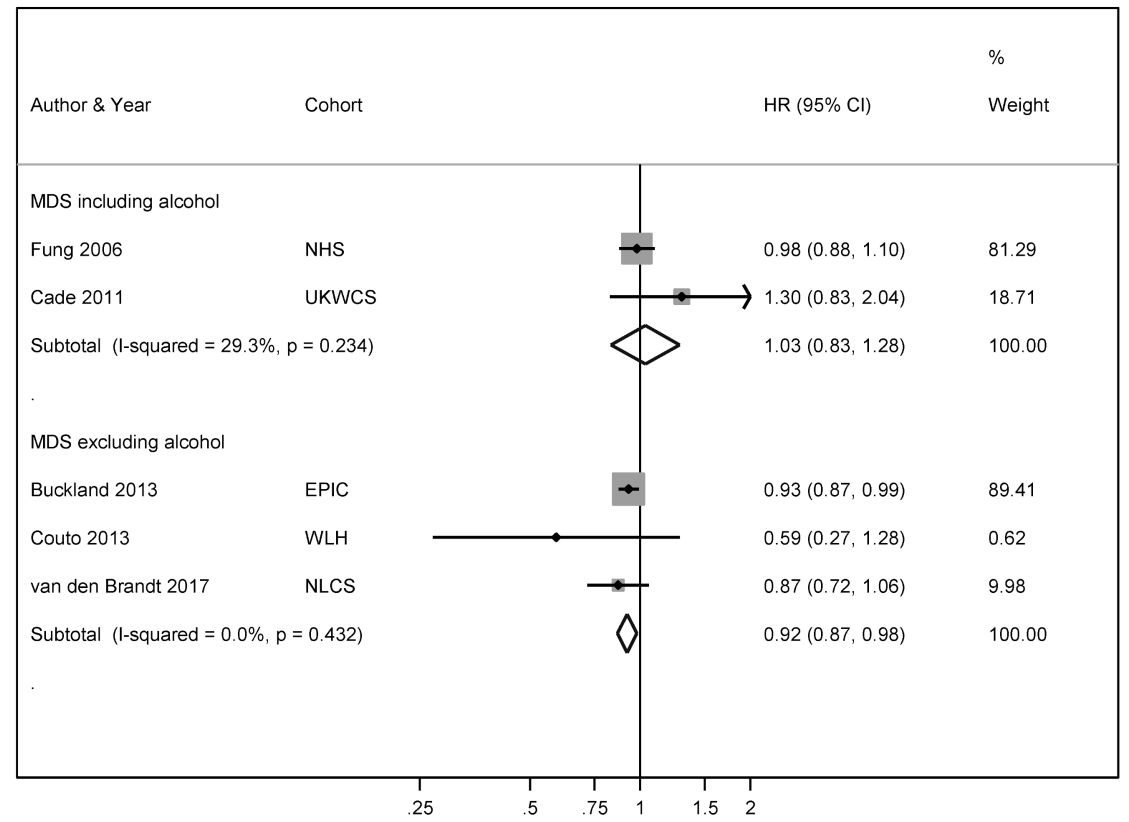

Supplementary Figure S3.2 Forest plots of postmenopausal breast cancer hazard ratios (HRs) and 95\% confidence intervals ( $95 \% \mathrm{Cls}$ ) comparing highest versus lowest category of adherence to $\mathrm{MD}$, from randomeffects meta-analyses

Separate plots are presented for studies that included alcohol in the MD-score, and those that excluded alcohol. Studies are referred to by first author, year of publication, and cohort abbreviation (EPIC: European Prospective Investigation into Cancer and Nutrition; NHS: Nurses' Health Study; NLCS: Netherlands Cohort Study; UKWCS: UK Women's Cohort Study; WLH: Women's Lifestyle and Health). Studies are weighted according to the inverse of the variance of the log HR estimate. The HRs are represented by the squares (the size is proportional to the weights used in the meta-analysis) and $95 \% \mathrm{Cls}$ are represented by the error bars. Diamonds represent the summary HR estimates and $95 \% \mathrm{Cls}$ per endpoint. 


\section{Chapter 4}

\section{Mediterranean diet adherence and risk of esophageal and gastric cancer subtypes in the Netherlands Cohort Study}

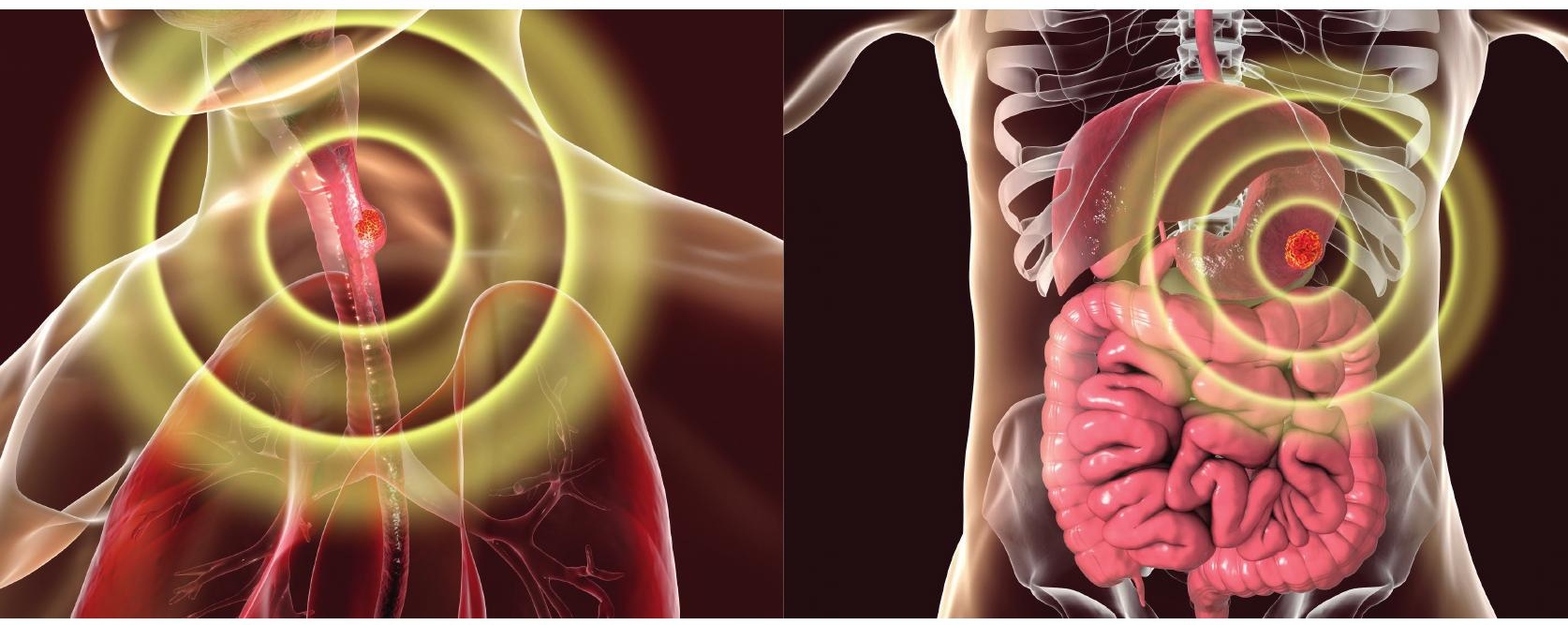

Maya Schulpen, Petra H. Peeters, Piet A. van den Brandt Gastric Cancer. 2019; 22(4): 663-674 


\begin{abstract}
Background

Mediterranean diet (MD) adherence has been associated with reduced risks of esophageal and gastric cancer (subtypes) in a limited number of studies. We prospectively investigated associations between MD adherence and risks of esophageal squamous cell carcinoma (ESCC), esophageal adenocarcinoma (EAC), gastric cardia adenocarcinoma (GCA) and gastric non-cardia adenocarcinoma (GNCA) in a Dutch cohort.
\end{abstract}

\title{
Methods
}

Analyses were conducted using data from the 120852 participants of the Netherlands Cohort Study (NLCS), who were aged between 55 and 69 years at enrollment. Various MD scores, with and without alcohol, were calculated to estimate MD adherence. Using 20.3 years of follow-up, 133 ESCC, 200 EAC, 191 GCA and 586 GNCA cases could be included in multivariable Cox regression analyses.

\section{Results}

Of the investigated scores, the alternate Mediterranean diet score without alcohol (aMEDr) performed best. aMEDr was inversely associated with risks of GCA and GNCA in men and women. However, statistical significance was only reached in men [ $p_{\text {trend }}: 0.019$ (GCA), 0.016 (GNCA)]. Furthermore, higher aMEDr values were significantly associated with a reduced ESCC risk in men $\left[\mathrm{HR}_{\text {per two-point increment }}(95 \% \mathrm{Cl})=0.57(0.41-0.80), \mathrm{p}_{\text {trend }}=0.013\right]$, but not in women $\left(p_{\text {heterogeneity }}=0.008\right)$. There was no evidence of an association between aMEDr and EAC risk. Educational level was a significant effect modifier for the association between aMEDr and GNCA risk ( $p_{\text {heterogeneity }}=0.0073$ ).

\section{Conclusions}

Higher MD adherence was associated with reduced risks of ESCC, GCA and GNCA in the NLCS. However, the decreased ESCC risk might be limited to men. 


\section{Introduction}

Cancers of the esophagus (sixth place) and stomach (third place) were amongst the most common causes of cancer-related death in the world in 2012 [1]. Two histologic types of esophageal cancer can be distinguished, namely esophageal squamous cell carcinoma (ESCC) and esophageal adenocarcinoma (EAC) [2]. Based on anatomic location, gastric cancers are subdivided into gastric cardia adenocarcinomas (GCA) and gastric non-cardia adenocarcinomas (GNCA) [3]. Different etiologies have been suggested for these subtypes $[2,3]$. In the past decades, incidence rates of EAC and GCA have been rising in many European countries and the United States (US) $[4,5]$.

The traditional Mediterranean diet (MD) is characterized by a high consumption of vegetables, fruits, whole grains and other plant foods, with olive oil as the principal source of fat. Foods from animal origin are consumed in low amounts in the MD, whereas alcohol intake is moderate [6-8]. The relation between a priori defined MD adherence and the incidence of esophageal and/or gastric cancer (subtypes) has been the topic of a limited number of studies [9-13]. In these studies, higher MD adherence has been associated with reduced risks of ESCC, GCA, GNCA and total gastric cancer (GC), but results were not always significant and sometimes inconsistent, primarily with respect to the gastric cancer subtypes [9-13].

Information bias due to reversed causation is a major concern when investigating relations between dietary factors and gastrointestinal cancer risk, because preclinical disease symptoms may cause patients with gastrointestinal tumors to alter their dietary habits already before clinical diagnosis. Another concern is recall bias, which could particularly be a problem in case-control studies. For these reasons, effects of dietary factors on gastrointestinal cancers should be investigated prospectively, if possible. So far, associations between MD adherence and risks of esophageal and/or gastric cancer subtypes have been prospectively investigated in only two cohort studies $[10,11]$. Therefore, more prospective evidence on this topic is desired.

This study prospectively investigated the association of $M D$ adherence with the risk of esophageal and gastric cancer subtypes (ESCC, EAC, GCA and GNCA) in the Netherlands Cohort Study (NLCS). We assessed MD adherence using various a priori defined MD scores, with and without alcohol, and examined associations for men and women separately.

\section{Methods}

\section{Study population and cancer follow-up}

The NLCS is a Dutch population-based cohort study, which has been described in detail previously [14-17]. In summary, the NLCS comprises 58279 men and 62573 women, aged 55 to 69 years, from 204 Dutch municipalities, who completed a self-administered questionnaire on diet and other cancer risk factors at baseline in September 1986. A case-cohort design was used to allow for efficient processing and analysis of the data. Therefore, a subcohort $(\mathrm{N}=5000)$ was randomly sampled immediately after baseline to estimate the number of person-years at risk. Cases were obtained from the total cohort. Vital status information for subcohort members was acquired biennially using municipal population registries $[14,17$, 18]. Approval for the NLCS was obtained from the institutional review boards of Maastricht University and the Netherlands Organization for Applied Scientific Research. All cohort 
members agreed to participate by filling out the questionnaire.

Follow-up for cancer incidence was carried out annually by record linkage with the Netherlands Cancer Registry and the Dutch Pathology Registry (PALGA) [15]. The NLCS cohort was followed up for 20.3 years until December $31^{\text {st }} 2006$. To be eligible for inclusion in the present study, esophageal and gastric cancer cases had to be incident and microscopically confirmed with known tumor histology and topography. Based on the International Classification of Diseases for Oncology, Third Edition, esophageal and gastric cancers were classified into ESCC (C15, histology codes: 8050-8076), EAC (C15, histology codes: 8140, $8141,8190-8231,8260-8263,8310,8430,8480-8490,8560$ and 8570-8572), GCA (C16.0) and GNCA (C16.1-C16.9). Subjects were excluded if they had prevalent cancer at baseline (except any type of skin cancer) and/or incomplete or inconsistent data on diet, alcohol or MD adherence. In total, 143 ESCC, 224 EAC, 218 GCA and 642 GNCA cases and 4084 subcohort members could be included in the analyses (Figure 4.1).

\section{Exposure assessment}

A self-administered, 150-item, semi-quantitative food frequency questionnaire (FFQ) was utilized to assess the participant's habitual diet during the year preceding baseline. The validity and reproducibility of this FFQ have been described previously [16, 19]. Mean daily nutrient intakes were calculated using the Dutch food composition table of the year 1986 [20].

\section{Mediterranean diet adherence}

MD adherence was measured using two variants of the traditional Mediterranean diet score (tMED) created by Trichopoulou et al., namely the alternate Mediterranean diet score (aMED) and the modified Mediterranean diet score (mMED) [21-25]. Differences in daily energy intakes were taken into account in the calculation of the MD scores by standardizing daily food intakes to 2000 (women) and 2500 (men) kilocalories [21, 25]. aMED assesses relative $M D$ adherence based on the mean daily intakes of nine dietary components [24, 25]. Each component is scored by 0 or 1 points with the maximum score of 9 representing the highest level of MD adherence. Subjects receive 1 point for intakes at or above the sexspecific median of vegetables (excluding potatoes), legumes, fruits, nuts, whole grains, fish and the ratio of monounsaturated to saturated fatty acids (MUFA:SFA ratio). The intake of red and processed meats is scored inversely. Additionally, 1 point is assigned to a moderate alcohol intake [5-25 grams per day (g/day) for both sexes] [24, 25]. mMED is calculated in a similar way as aMED, but includes slightly different dietary components [23]. In mMED, intakes of fruits and nuts are grouped together and total cereal and meat intake is scored. Additionally, 1 point is obtained for a dairy intake below the sex-specific median. To improve the usage of mMED in non-Mediterranean populations, the fatty acid quality is measured by the ratio of unsaturated fatty acids (MUFA + polyunsaturated fatty acids) to SFA. Finally, a moderate alcohol intake is defined differently for men (10-50 g/day) and women (5-25 g/ day) [23]. (Heavy) alcohol consumption has been associated with an increased risk of ESCC and probably GC [26]. Therefore, MD adherence was also assessed using aMED and mMED variants without alcohol (aMEDr and mMEDr, respectively), which had maximum scores of 8 points. 


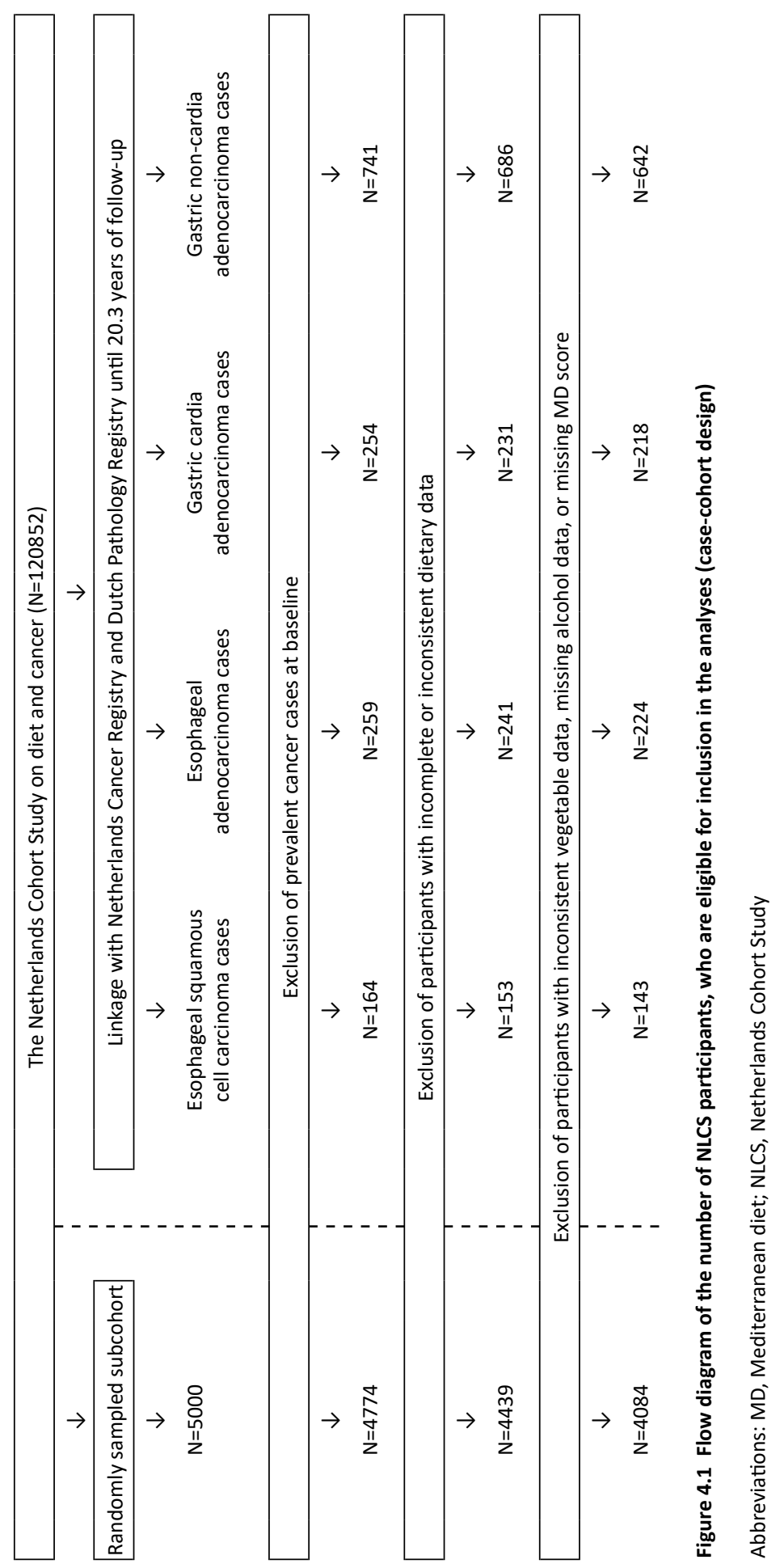




\section{Statistical analyses}

All analyses were performed separately for men and women, unless otherwise specified. Hazard ratios (HRs) and $95 \%$ confidence intervals (95\% Cls) for associations of MD adherence with incidence of esophageal and gastric cancer subtypes were estimated using Cox proportional hazards models with follow-up duration as time variable. Person-years at risk for subcohort members were calculated from baseline until diagnosis of esophageal or gastric cancer, death, emigration, loss to follow-up or end of follow-up, whichever came first. Standard errors were estimated using the Huber-White sandwich estimator to account for the increased variance because of subcohort sampling [27]. To verify that all variables met the proportional hazards assumption, scaled Schoenfeld residuals tests and $-\ln (-\ln )$ survival plots were used [28]. A time-varying covariate was included in the model when a potential confounder violated the proportional hazards assumption and inclusion of a timevarying covariate altered the HR of the MD score.

MD scores were included as categorical [low: 0-3, middle: 4-5, high: 6-8(9)] and continuous (per two-point increment) terms in age-adjusted and fully adjusted analyses [23, 25]. Tests for trends were performed by assigning sex-specific median values among subcohort members to the MD score categories and fitting these as continuous terms in the Cox regression models. To correct for potential confounding, the following set of literature-selected variables was included in fully adjusted Cox models: age at baseline, sex (except for sexspecific models), cigarette smoking status, cigarette smoking frequency, cigarette smoking duration, body mass index (BMI), total daily energy intake, alcohol consumption (except for models containing original MD scores including alcohol), highest level of education, nonoccupational physical activity and family history of esophageal cancer (for ESCC and EAC) or gastric cancer (for GCA and GNCA).

Akaike's Information Criterion (AIC) was used to compare performances of models containing aMEDr and mMEDr [29]. Furthermore, it was evaluated if inclusion of alcohol in the MD scores affected the model fits. Considering that (heavy) alcohol consumption has been associated with an increased risk of ESCC and probably GC, MD scores without alcohol are prioritized in the Results section of this article and subsequent analyses were only performed using aMEDr [26]. Moreover, we prefer the use of aMEDr to assess MD adherence, because aMEDr-containing models had similar or better performances than mMEDr-containing models in the NLCS, both in the present and earlier analyses [30, 31].

The relative importance of the individual aMEDr components was investigated in two ways. First, all aMEDr components were entered simultaneously as dichotomous variables into fully adjusted Cox models. Second, HRs per two-point increment were estimated upon alternate removal of each aMEDr component from the sum score, one at a time, using the method described by Trichopoulou et al. [32]. In addition, analyses stratified by cigarette smoking status, alcohol consumption, BMI and educational level were performed. The statistical significance of possible differences across strata was tested by including interaction terms between aMEDr and the potential effect modifiers. Finally, sensitivity analyses were performed in which the first two years of follow-up were excluded. Men and women were combined in the stratified and sensitivity analyses to increase the power. All statistical analyses were conducted using Stata version 15 (StataCorp LLC, College Station, TX, USA). Reported $p$-values are two-sided and $p$-values below 0.05 were considered statistically significant. 


\section{Results}

Sex-specific baseline characteristics of cases and subcohort members are presented in Table 4.1. Male ESCC, GCA, and GNCA cases had lower MD adherence than subcohort members. No clear differences in MD adherence were observed between female cases and subcohort members. Concerning potential confounding factors, cases were older (except for male ESCC and EAC cases) and less often never smokers (except for female EAC cases) than subcohort members. In addition, alcohol consumption was higher in ESCC and GCA (men only) cases, but lower in EAC cases (women only). Finally, the mean BMI was lower in ESCC cases, but higher in EAC and GCA cases.

Tables 4.2 (men) and 4.3 (women) show fully adjusted associations between MD adherence, assessed using various MD scores, and the risk of esophageal and gastric cancer subtypes. Not all eligible study participants could be included in the Cox models because of missing values in covariates. Results of the age-adjusted analyses are presented in Supplementary Table S4.1.

High MD adherence according to aMEDr was associated with significantly reduced risks of ESCC, GCA and GNCA in men $\left[\mathrm{HR}_{\text {high vs. low }}(95 \% \mathrm{Cl})\right.$ : ESCC $=0.35(0.14-0.89), \mathrm{GCA}=0.48(0.26-$ $0.89), \mathrm{GNCA}=0.65(0.45-0.94)]$ with significant tests for trends. In women, associations of aMEDr with GCA and GNCA risk were also inverse, but did not reach statistical significance $\left[\mathrm{HR}_{\text {per two-point increment }}(95 \% \mathrm{Cl}): \mathrm{GCA}=0.82(0.51-1.33), \mathrm{GNCA}=0.83(0.67-1.01)\right]$. In contrast to men, aMEDr was not inversely associated with ESCC risk in women. EAC risk was not associated with aMEDr in both sexes. Heterogeneity tests showed that the association of aMEDr with ESCC risk differed significantly between the sexes $\left(p_{\text {heterogeneity }}=0.008\right)$. Associations of similar directions were observed when MD adherence was assessed using mMEDr in men, whereas we did not observe associations with any of the subtypes in women using mMEDr.

Overall, vegetable and fruit intakes were strong contributors to the inverse associations observed in male NLCS participants. High nut intake was associated with a significantly reduced GNCA risk $(p=0.008)$ in men, but did not contribute to the inverse association with ESCC risk. Furthermore, a low intake of red and processed meats contributed considerably to the inverse association with ESCC risk in men. Concerning the non-significant inverse associations with GCA and GNCA risk observed in women, intakes of nuts, whole grains, fish and the MUFA:SFA ratio particularly contributed. In women, a high fish intake was associated with a significantly reduced GCA risk $(p=0.046)$ (data not shown).

Based on AIC values, models containing aMEDr performed similarly or better than mMEDrcontaining models for all esophageal and gastric cancer subtypes considered. Inclusion of alcohol in aMED resulted in a clearly worse model fit when considering ESCC risk. Similar model performances were observed when aMED variants with and without alcohol were compared for EAC, GCA and GNCA risk.

In the stratified analyses (Table 4.4), men and women were combined to increase the statistical power. Associations of aMEDr with all esophageal and gastric cancer subtypes were similar across strata of cigarette smoking status, alcohol consumption and BMI. A significant interaction between aMEDr and level of education was observed for GNCA risk $\left(p_{\text {heterogeneity }}=0.0073\right)$ with a significant inverse association only being present in the lowest category. Inverse associations were also most apparent among those in the lowest education category when considering ESCC and GCA, but interaction tests were not significant for these subtypes. Finally, exclusion of the first two years of follow-up did not relevantly change the 
results (data not shown).

\section{Discussion}

In the NLCS, higher aMEDr values were associated with significantly reduced risks of ESCC, GCA and GNCA in men. In women, we observed non-significant inverse associations between aMEDr and risks of GCA and GNCA, but not ESCC. MD adherence was not associated with EAC risk. Associations of aMEDr with ESCC risk significantly differed between the sexes. Compared to mMEDr-containing models, aMEDr-containing models had similar or better performances. Model performances were generally comparable for aMED variants with and without alcohol, except for ESCC, were models containing aMED (including alcohol) clearly fitted worse.

Results of previously conducted prospective cohort studies [10, 11] investigating the association of a priori defined MD adherence with the risk of esophageal and/or gastric cancer subtypes were partially in accordance with our observations in the NLCS. In the US National Institutes of Health-American Association of Retired Persons Diet and Health study, high MD adherence (aMED) was associated with a significantly reduced risk of ESCC, but not EAC, GCA and GNCA [11]. Although not statistically significant, the association with GNCA was also inverse. In the European Prospective Investigation into Cancer and Nutrition, inverse associations with $\mathrm{MD}$ adherence (relative Mediterranean diet score including alcohol, rMED) were suggested for total GC, GCA and GNCA, but only reached statistical significance for total GC and GCA [10]. In accordance with the cohort evidence, an Italian case-control study also observed a significant inverse association between a priori defined MD adherence and ESCC risk [9]. Case-control studies focusing on gastric cancer risk found higher MD adherence ( $a$ priori defined) to be associated with significantly reduced risks of GCA, GNCA and total GC $[12,13]$. Finally, adherence to an a posteriori defined MD pattern was inversely associated with total GC risk in a Spanish case-control study [33]. Subtypespecific analyses showed that the inverse association was only statistically significant for GNCA. Combining male and female participants in the present study, $\mathrm{HRs}(95 \% \mathrm{Cl}$ ) per twopoint increment in aMEDr were $0.77(0.61-0.98)$ for ESCC, $1.14(0.94-1.37)$ for EAC, 0.86 $(0.71-1.04)$ for GCA and $0.83(0.73-0.93)$ for GNCA.

For ESCC, associations with aMEDr significantly differed between male and female NLCS participants. Prior studies by Bosetti et al. [9] and Li et al. [11] did not observe a significant interaction between sex and MD adherence for ESCC risk. However, the inverse association in the latter study also seemed to be restricted to men [11]. Residual confounding by smoking behavior could potentially have caused the inverse association between aMEDr and ESCC risk that we observed in men. Tobacco smoking is strongly associated with an increased risk of ESCC $[2,34]$. In our study, subjects in the highest aMEDr category were less likely to be current smokers. Since, male participants were more likely to smoke than female participants, the effect of residual confounding by smoking behavior would be larger in men. This could potentially explain why we only observed an inverse association between aMEDr and ESCC risk in men. However, additional subgroup analyses for smoking status restricted to men showed that the inverse association between aMEDr and ESCC risk was strongest in men who had never smoked (data not shown), making it less likely that the observed differences between men and women in the NLCS were solely due to residual confounding by smoking behavior. Therefore, potential male-female differences in the association of MD adherence with ESCC risk deserve attention in future studies. For EAC, GCA, GNCA and total 
GC there was no evidence of heterogeneity between the sexes in neither the present study nor the literature [10-13].

In the present study, inverse associations between aMEDr and ESCC, GCA and GNCA risk were most pronounced in subjects in the lowest education category with a significant interaction being observed for GNCA. Similarly, Li et al. [11] reported that aMED was only significantly inversely associated with EAC risk in the lowest education category $\left(p_{\text {interaction }}=0.02\right)$. However, there was no evidence of effect modification by educational level in the study by Praud et al. [12]. Although the interaction between aMEDr and educational level that we observed might be a chance finding due to the large number of tests performed, it should be investigated in future studies.

In their Third Expert Report, the World Cancer Research Fund/American Institute for Cancer Research suggested that high intakes of vegetables and fruits, and low intakes of processed meat and grilled (broiled) or barbecued (charbroiled) meat and fish might be associated with reduced risks of ESCC, EAC and/or GC [26]. This is in correspondence with our observations that high intakes of vegetables, fruits and nuts, and a low intake of red and processed meats (ESCC only) particularly contributed to the inverse associations with ESCC, GCA and GNCA risk in men in the NLCS. In women, nuts, whole grains, fish and the MUFA:SFA ratio were important aMEDr components contributing to the non-significant inverse associations observed for GCA and GNCA risk. Inverse associations (not all significant) between esophageal and/or gastric cancer subtypes and intakes of nuts (ESCC and GNCA), vegetables (ESCC and EAC) and fruits (ESCC) were also documented in previous NLCS analyses [35, 36]. Additionally, high intakes of red (non-significant) and processed meats were associated with an increased ESCC risk in men [37]. Although the abovementioned aMEDr components were important contributors to the inverse associations that we observed in the present analysis, none of the individual components seemed to be the sole driver. This supports our patternbased approach, which accounts for synergistic and antagonistic interactions between dietary components and solves collinearity and confounding issues associated with the evaluation of individual components. Moreover, weak effects of single dietary components may only emerge when combined in dietary patterns $[22,38,39]$.

Different etiologies have been suggested for the subtypes of esophageal and gastric cancer based on differences in risk factors and incidence trends $[2,3,34,40]$. This stresses the importance of considering ESCC, EAC, GCA and GNCA as separate outcomes as we did in this study. ESCC and EAC were clearly differently associated with MD adherence in our analysis. While MD adherence was significantly inversely associated with ESCC risk in men, no association was observed with EAC risk. In contrast to the esophageal cancer subtypes, GCA and GNCA seemed to have roughly comparable associations with MD adherence.

MD adherence might reduce cancer risk by decreasing oxidative stress, reactive oxygen species-induced DNA damage and inflammation [39, 41]. The MD is rich in antioxidants (e.g., vitamins and polyphenols) from plant foods and olive oil, and has been associated with higher total antioxidant capacity and lower levels of oxidized low-density lipoprotein cholesterol $[26,39,41,42]$. Moreover, polyphenols (e.g., flavonoids) may reduce inflammation and MD adherence has been inversely associated with inflammatory biomarker concentrations [24, 43]. Finally, dietary fiber possibly acts as a nitrite scavenger, counteracting the carcinogenic effects of $\mathrm{N}$-nitroso compounds $[39,44]$. Low meat intake may also contribute to the cancerprotective effects of the MD. Nitrates and nitrites in processed meat can form N-nitroso compounds in the stomach. Besides, haem iron in red meat also stimulates the endogenous 


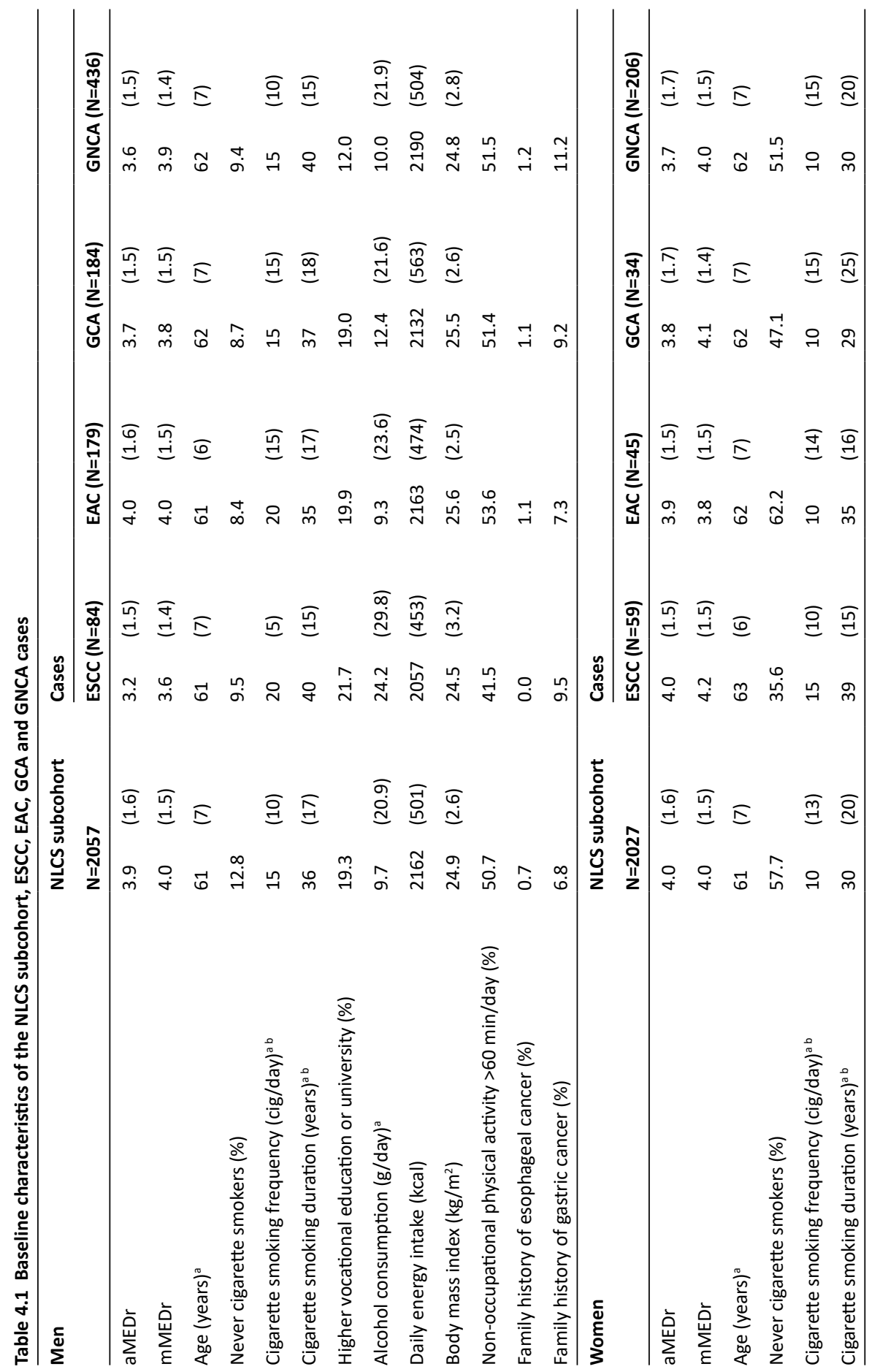




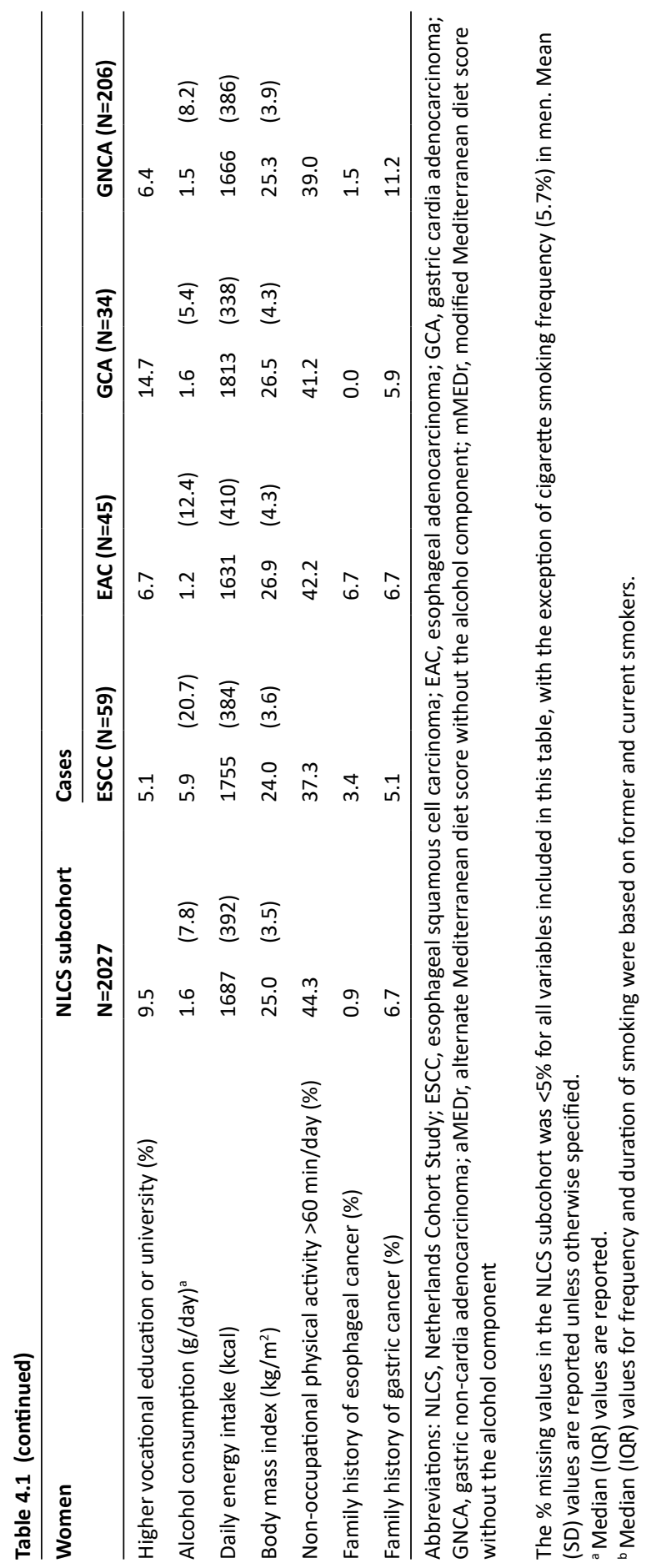




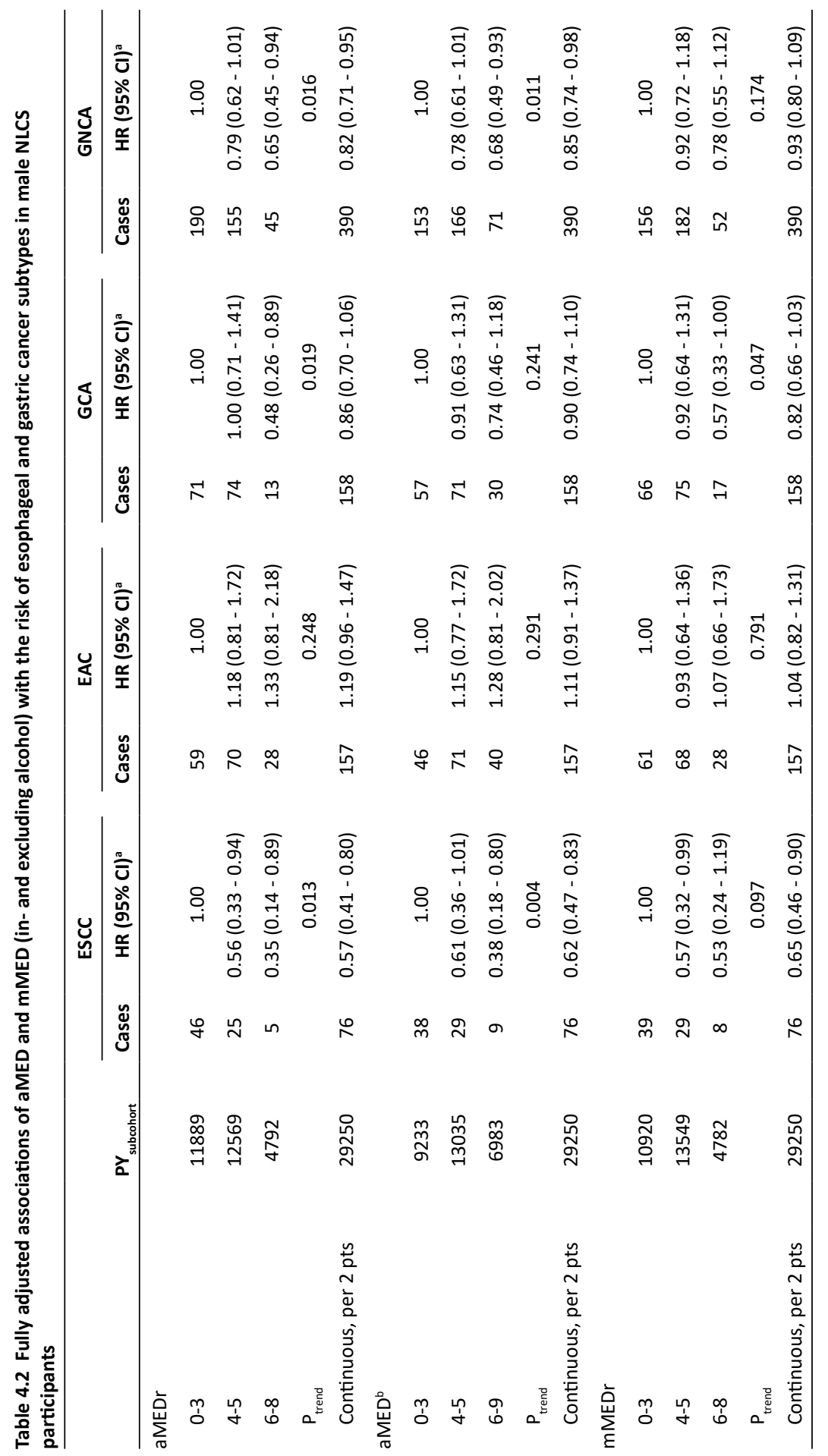




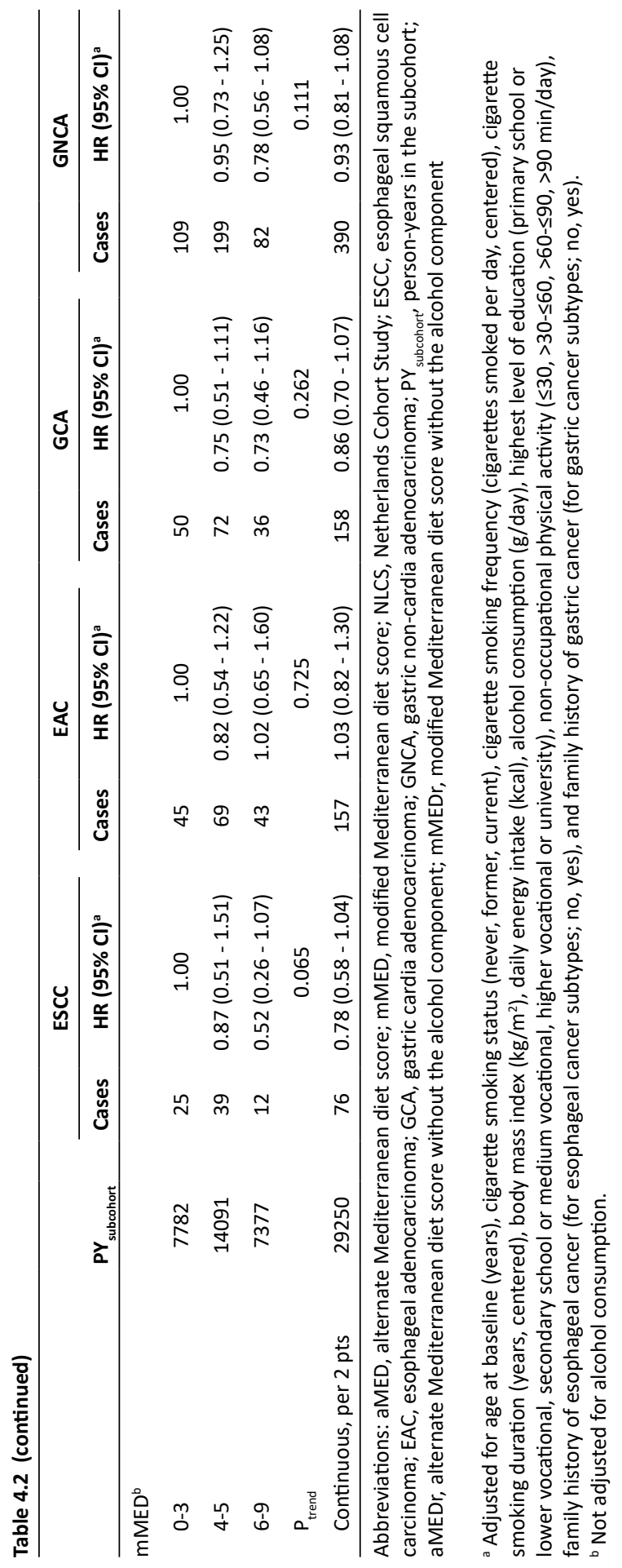




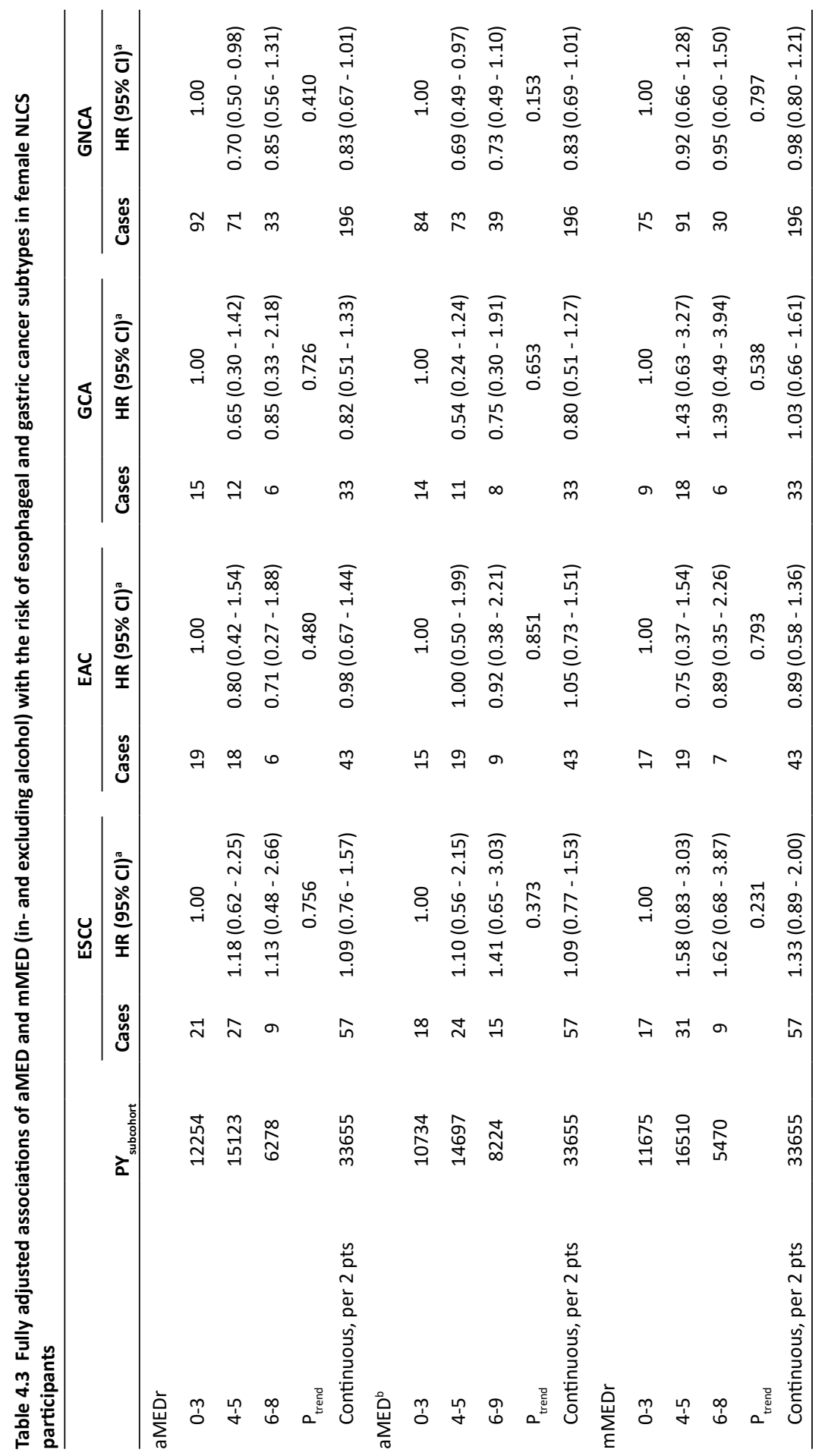




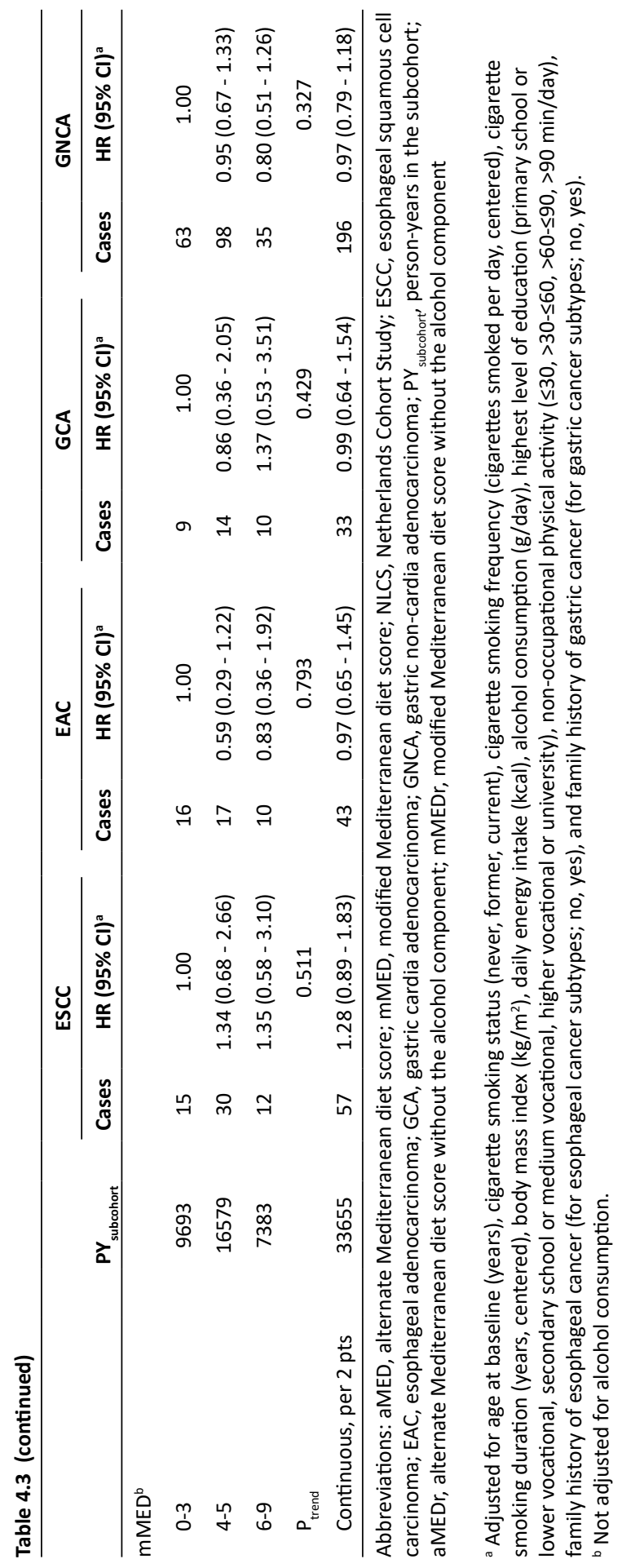




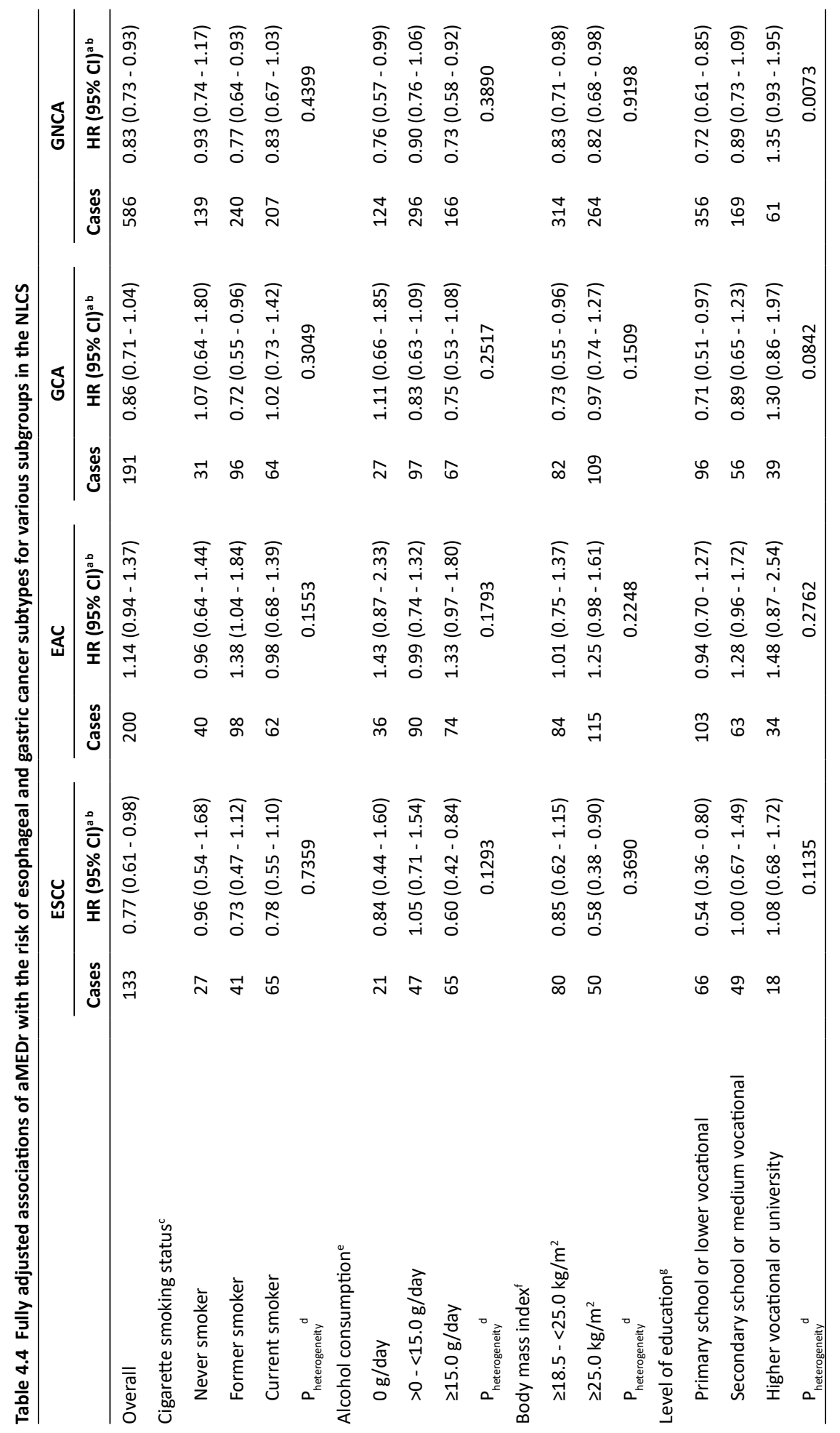




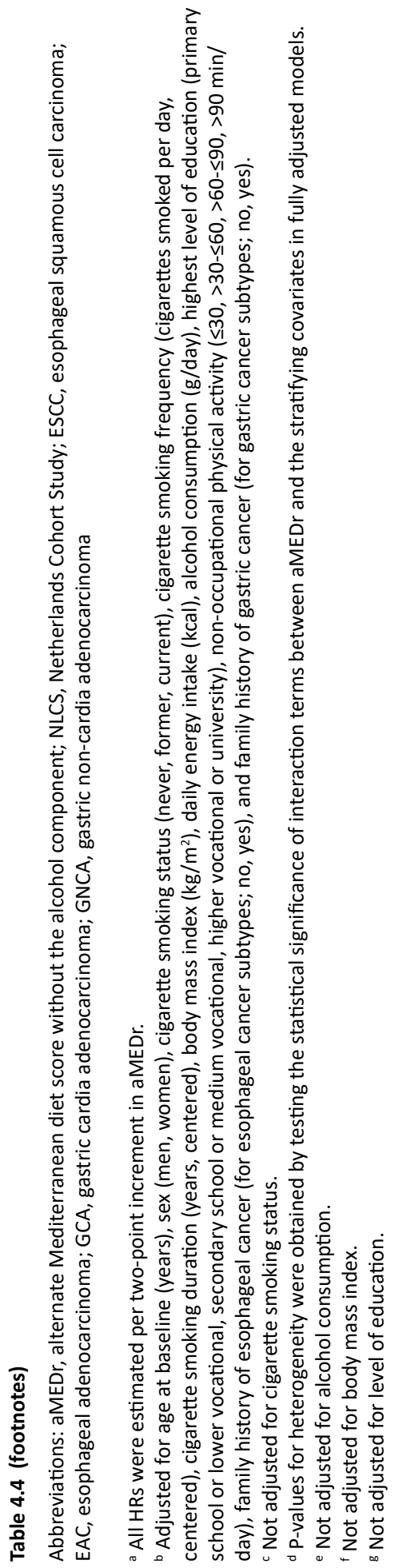


formation of N-nitroso compounds and causes oxidative stress and DNA damage. Finally, carcinogenic heterocyclic amines and polycyclic aromatic hydrocarbons are formed during high-temperature cooking of meat [26].

The use of data from a large prospective cohort with a long duration of follow-up enabled us to perform subtype- and sex-specific analyses. However, case numbers for the individual subtypes were low, necessitating us to combine men and women in the stratified and sensitivity analyses to increase the statistical power. Another strength of our study was the availability of high quality dietary data. The NLCS-FFQ was validated using nine-day dietary records completed over three different seasons, showing an adequate performance [16]. Furthermore, a reproducibility study demonstrated that the single baseline measurement of this FFQ performed relatively well in ranking subjects according to their nutrient intake for over at least five years [19]. Nonetheless, changes in dietary habits and potential confounders during follow-up might still have attenuated the observed associations. Instead of using self-reporting based methods (e.g., FFQs, dietary records), dietary intake could be assessed by measurement of biomarker concentrations in blood. However, there are currently no biomarkers available that assess adherence to all aspects of the MD. Furthermore, biomarker levels in blood are also influenced by, e.g., absorption and excretion rates and reflect only short term dietary intake. Despite the fact that we adjusted for a large number of potential confounders, residual confounding by unmeasured factors may still exist. For example, we did not obtain data regarding Helicobacter pylori infection, which might have confounded our results in particular for GNCA. Furthermore, we cannot exclude the possibility of errors in the outcome measurements. However, it was reported that the histology and topography information from the Netherlands Cancer Registry, which we used to define the tumor subtypes, is of high accuracy [45]. Reversed causation due to the presence of preclinical disease symptoms in cases is another concern, particularly when investigating relations between dietary factors and gastrointestinal cancers, as we discussed previously [46]. Prospective cohort studies are less sensitive to this type of bias than case-control designs and we obtained similar results when excluding the first two years of follow-up. Data on the reliability of $a$ priori scores used in the assessment of MD adherence are limited [47]. The reliability of ten indexes measuring MD adherence, including mMED and rMED, has been evaluated by assessing correlations with a hidden common factor (obtained by factor analysis) understood as "MD adherence" [48]. Both mMED and rMED were amongst the four indexes that showed high correlations with the "MD adherence" factor (mMED: 0.83, rMED: 0.80) [48]. Regarding the validity of the MD scores used, subjects with higher MD score values in our study consumed, as expected, more plant foods (e.g., vegetables, legumes, fruits and nuts) and less foods from animal origin (e.g., meat and dairy products). A similar pattern was previously observed for tMED [22]. However, the validity of tMED and its variants has mainly been established by showing inverse associations with various adverse health outcomes including all-cause mortality and risks of and mortality from cardiovascular diseases and cancer, e.g., $[22,23,25,49,50]$. Several studies have compared associations between various index-based dietary patterns, including tMED (variants), and cancer risk. A review published in 2018 showed that 3 out of 4 studies investigating the association of tMED (variants) with postmenopausal estrogen receptor-negative breast cancer risk observed a significant inverse relation. However, associations were inconsistent for other dietary pattern scores [e.g., Dietary Inflammatory Index (DII), Healthy Eating Index (HEI) and Alternate Healthy Eating Index (AHEI)] [51]. Considering prostate cancer risk, tMED (variants) and DII showed relatively consistent associations across studies, whereas the 
evidence was inconsistent and/or insufficient for other indexes [52]. Furthermore, healthier diets according to tMED (variants), HEI/AHEI and DII have all been associated with lower risks of colorectal cancer [53]. More studies evaluating associations of various index-based dietary patterns with cancer risk are required in order to identify the preferred dietary pattern(s) in the perspective of cancer prevention. A final limitation of our study was the population-dependent assignment of scores in the assessment of MD adherence, which we have elaborately discussed previously [46].

In conclusion, high MD adherence was associated with reduced risks of ESCC, GCA and GNCA in the NLCS. However, the inverse association with ESCC risk seemed to be restricted to men. So far, results for esophageal cancer generally were consistent with high MD adherence being associated with a reduced risk of ESCC, but not EAC. Findings concerning GCA and GNCA were more diverse, but generally, inverse associations (not always significant) were observed for at least one of the subtypes. The potential differences in associations between men and women, particularly for ESCC, require further attention. 


\section{References}

1. Ferlay J, Soerjomataram I, Ervik M, Dikshit R, Eser S, Mathers C, et al. GLOBOCAN 2012 v1.0, Cancer Incidence and Mortality Worldwide: IARC CancerBase No. 11 [Internet]. Lyon, France: International Agency for Research on Cancer; 2013 [cited 2018 July]. Available from: http://globocan. iarc.fr.

2. Kamangar F, Chow WH, Abnet CC, Dawsey SM. Environmental causes of esophageal cancer. Gastroenterol Clin North Am. 2009;38(1):27-57, vii.

3. Crew KD, Neugut Al. Epidemiology of gastric cancer. World J Gastroenterol. 2006;12(3):354-

62.

4. Brown LM, Devesa SS. Epidemiologic trends in esophageal and gastric cancer in the United States. Surg Oncol Clin N Am. 2002;11(2):235-56.

5. Steevens J, Botterweck AA, Dirx MJ, van den Brandt PA, Schouten LJ. Trends in incidence of oesophageal and stomach cancer subtypes in Europe. Eur J Gastroenterol Hepatol. 2010;22(6):669-78.

6. Willett WC, Sacks F, Trichopoulou A, Drescher G, Ferro-Luzzi A, Helsing E, et al. Mediterranean diet pyramid: a cultural model for healthy eating. Am J Clin Nutr. 1995;61(6 Suppl):1402S-6S.

7. Trichopoulou A, Lagiou P. Healthy traditional Mediterranean diet: an expression of culture, history, and lifestyle. Nutr Rev. 1997;55(11 Pt 1):383-9.

8. Fung TT, Rexrode KM, Mantzoros CS, Manson JE, Willett WC, Hu FB. Mediterranean diet and incidence of and mortality from coronary heart disease and stroke in women. Circulation. 2009;119(8):1093-100.

9. Bosetti C, Gallus S, Trichopoulou A, Talamini R, Franceschi S, Negri E, et al. Influence of the Mediterranean diet on the risk of cancers of the upper aerodigestive tract. Cancer Epidemiol Biomarkers Prev. 2003;12(10):1091-4.

10. Buckland G, Agudo A, Lujan L, Jakszyn P, Bueno-de-Mesquita HB, Palli D, et al. Adherence to a Mediterranean diet and risk of gastric adenocarcinoma within the European Prospective Investigation into Cancer and Nutrition (EPIC) cohort study. Am J Clin Nutr. 2010;91(2):381-90.

11. Li WQ, Park Y, Wu JW, Ren JS, Goldstein AM, Taylor PR, et al. Index-based dietary patterns and risk of esophageal and gastric cancer in a large cohort study. Clin Gastroenterol Hepatol. 2013;11(9):1130-6 e2.

12. Praud D, Bertuccio P, Bosetti C, Turati F, Ferraroni M, La Vecchia C. Adherence to the Mediterranean diet and gastric cancer risk in Italy. Int J Cancer. 2014;134(12):2935-41.

13. Stojanovic J, Giraldi L, Arzani D, Pastorino R, Biondi A, Persiani R, et al. Adherence to Mediterranean diet and risk of gastric cancer: results of a case-control study in Italy. Eur J Cancer Prev. 2017;26(6):491-6.

14. van den Brandt PA, Goldbohm RA, van 't Veer P, Volovics A, Hermus RJ, Sturmans F. A largescale prospective cohort study on diet and cancer in The Netherlands. J Clin Epidemiol. 1990;43(3):28595.

15. van den Brandt PA, Schouten LJ, Goldbohm RA, Dorant E, Hunen PM. Development of a record linkage protocol for use in the Dutch Cancer Registry for Epidemiological Research. Int J Epidemiol. 1990;19(3):553-8.

16. Goldbohm RA, van den Brandt PA, Brants HA, van 't Veer P, Al M, Sturmans F, et al. Validation of a dietary questionnaire used in a large-scale prospective cohort study on diet and cancer. Eur J Clin Nutr. 1994;48(4):253-65.

17. Volovics A, van den Brandt PA. Methods for the analyses of case-cohort studies. Biometrical J. 1997;39(2):195-214.

18. Prentice RL. A case-cohort design for epidemiologic cohort studies and disease prevention trials. Biometrika. 1986;73(1):1-11.

19. Goldbohm RA, van 't Veer P, van den Brandt PA, van 't Hof MA, Brants HA, Sturmans F, et al. Reproducibility of a food frequency questionnaire and stability of dietary habits determined from five annually repeated measurements. Eur J Clin Nutr. 1995;49(6):420-9.

20. NEVO table. Dutch food composition table 1986-1987. The Hague, the Netherlands: Voorlichtingsbureau voor de Voeding; 1986.

21. Trichopoulou A, Kouris-Blazos A, Wahlqvist ML, Gnardellis C, Lagiou P, Polychronopoulos E, et al. Diet and overall survival in elderly people. BMJ. 1995;311(7018):1457-60. 
22. Trichopoulou A, Costacou T, Bamia C, Trichopoulos D. Adherence to a Mediterranean diet and survival in a Greek population. N Engl J Med. 2003;348(26):2599-608.

23. Trichopoulou A, Orfanos P, Norat T, Bueno-de-Mesquita B, Ocke MC, Peeters PH, et al. Modified Mediterranean diet and survival: EPIC-elderly prospective cohort study. BMJ. 2005;330(7498):991.

24. Fung TT, McCullough ML, Newby PK, Manson JE, Meigs JB, Rifai N, et al. Diet-quality scores and plasma concentrations of markers of inflammation and endothelial dysfunction. Am J Clin Nutr. 2005;82(1):163-73.

25. Mitrou PN, Kipnis V, Thiebaut AC, Reedy J, Subar AF, Wirfalt E, et al. Mediterranean dietary pattern and prediction of all-cause mortality in a US population: results from the NIH-AARP Diet and Health Study. Arch Intern Med. 2007;167(22):2461-8.

26. World Cancer Research Fund / American Institute for Cancer Research. Diet, nutrition, physical activity and cancer: a global perspective. Continuous update project expert report 2018. 2018. Available from: https://www.wcrf.org/dietandcancer.

27. Lin DY, Wei LJ. The Robust Inference for the Cox Proportional Hazards Model. J Am Stat Assoc. 1989;84(408):1074-8.

28. Grambsch PM, Therneau TM. Proportional Hazards Tests and Diagnostics Based on Weighted Residuals. Biometrika. 1994;81(3):515-26.

29. Akaike H. A new look at the statistical model identification. IEEE Transactions on automatic control. 1974;AC-19(6):716-23.

30. van den Brandt PA, Schulpen M. Mediterranean diet adherence and risk of postmenopausal breast cancer: results of a cohort study and meta-analysis. Int J Cancer. 2017;140(10):2220-31.

31. Schulpen $M$, van den Brandt PA. Adherence to the Mediterranean diet and risk of lung cancer in the Netherlands Cohort Study. Br J Nutr. 2018;119(6):674-84.

32. Trichopoulou A, Bamia C, Trichopoulos D. Anatomy of health effects of Mediterranean diet: Greek EPIC prospective cohort study. BMJ. 2009;338:b2337.

33. Castello A, Fernandez de Larrea N, Martin V, Davila-Batista V, Boldo E, Guevara M, et al. High adherence to the Western, Prudent, and Mediterranean dietary patterns and risk of gastric adenocarcinoma: MCC-Spain study. Gastric Cancer. 2018;21(3):372-82.

34. Domper Arnal MJ, Ferrandez Arenas A, Lanas Arbeloa A. Esophageal cancer: Risk factors, screening and endoscopic treatment in Western and Eastern countries. World J Gastroenterol. 2015;21(26):7933-43.

35. Steevens J, Schouten LJ, Goldbohm RA, van den Brandt PA. Vegetables and fruits consumption and risk of esophageal and gastric cancer subtypes in the Netherlands Cohort Study. Int J Cancer. 2011;129(11):2681-93.

36. Nieuwenhuis L, van den Brandt PA. Tree nut, peanut, and peanut butter consumption and the risk of gastric and esophageal cancer subtypes: the Netherlands Cohort Study. Gastric Cancer. 2018;21(6):900-12.

37. Keszei AP, Schouten LJ, Goldbohm RA, van den Brandt PA. Red and processed meat consumption and the risk of esophageal and gastric cancer subtypes in The Netherlands Cohort Study. Ann Oncol. 2012;23(9):2319-26.

38. Jacques PF, Tucker KL. Are dietary patterns useful for understanding the role of diet in chronic disease? Am J Clin Nutr. 2001;73(1):1-2.

39. Verberne L, Bach-Faig A, Buckland G, Serra-Majem L. Association between the Mediterranean diet and cancer risk: a review of observational studies. Nutr Cancer. 2010;62(7):860-70.

40. Jemal A, Center MM, DeSantis C, Ward EM. Global patterns of cancer incidence and mortality rates and trends. Cancer Epidemiol Biomarkers Prev. 2010;19(8):1893-907.

41. Brill JB. The Mediterranean diet and your health. Am J Lifestyle Med. 2009;3(1):44-56.

42. Pitsavos C, Panagiotakos DB, Tzima N, Chrysohoou C, Economou M, Zampelas A, et al. Adherence to the Mediterranean diet is associated with total antioxidant capacity in healthy adults: the ATTICA study. Am J Clin Nutr. 2005;82(3):694-9.

43. Rahman I, Biswas SK, Kirkham PA. Regulation of inflammation and redox signaling by dietary polyphenols. Biochem Pharmacol. 2006;72(11):1439-52.

44. Moller ME, Dahl R, Bockman OC. A possible role of the dietary fibre product, wheat bran, as a nitrite scavenger. Food Chem Toxicol. 1988;26(10):841-5.

45. Schouten LJ, Jager JJ, van den Brandt PA. Quality of cancer registry data: a comparison of 


\section{Chapter 4}

data provided by clinicians with those of registration personnel. $\mathrm{Br} J$ Cancer. 1993;68(5):974-7.

46. Schulpen $\mathrm{M}$, Peeters PH, van den Brandt PA. Mediterranean diet adherence and risk of pancreatic cancer: A pooled analysis of two Dutch cohorts. Int J Cancer. 2019;144(7):1550-60.

47. Zaragoza-Marti A, Cabanero-Martinez MJ, Hurtado-Sanchez JA, Laguna-Perez A, FerrerCascales R. Evaluation of Mediterranean diet adherence scores: a systematic review. BMJ Open. 2018;8(2):e019033.

48. Mila-Villarroel R, Bach-Faig A, Puig J, Puchal A, Farran A, Serra-Majem L, et al. Comparison and evaluation of the reliability of indexes of adherence to the Mediterranean diet. Public Health Nutr. 2011;14(12A):2338-45.

49. Couto E, Boffetta P, Lagiou P, Ferrari P, Buckland G, Overvad K, et al. Mediterranean dietary pattern and cancer risk in the EPIC cohort. Br J Cancer. 2011;104(9):1493-9.

50. Buckland G, Gonzalez CA, Agudo A, Vilardell M, Berenguer A, Amiano P, et al. Adherence to the Mediterranean diet and risk of coronary heart disease in the Spanish EPIC Cohort Study. Am J Epidemiol. 2009;170(12):1518-29.

51. Du M, Liu SH, Mitchell C, Fung TT. Associations between Diet Quality Scores and Risk of Postmenopausal Estrogen Receptor-Negative Breast Cancer: A Systematic Review. J Nutr. 2018;148(1):100-8.

52. Kim JH, Kim J. Index-Based Dietary Patterns and the Risk of Prostate Cancer. Clin Nutr Res. 2017;6(4):229-46.

53. Steck SE, Guinter M, Zheng J, Thomson CA. Index-based dietary patterns and colorectal cancer risk: a systematic review. Adv Nutr. 2015;6(6):763-73. 


\section{Supplementary material}

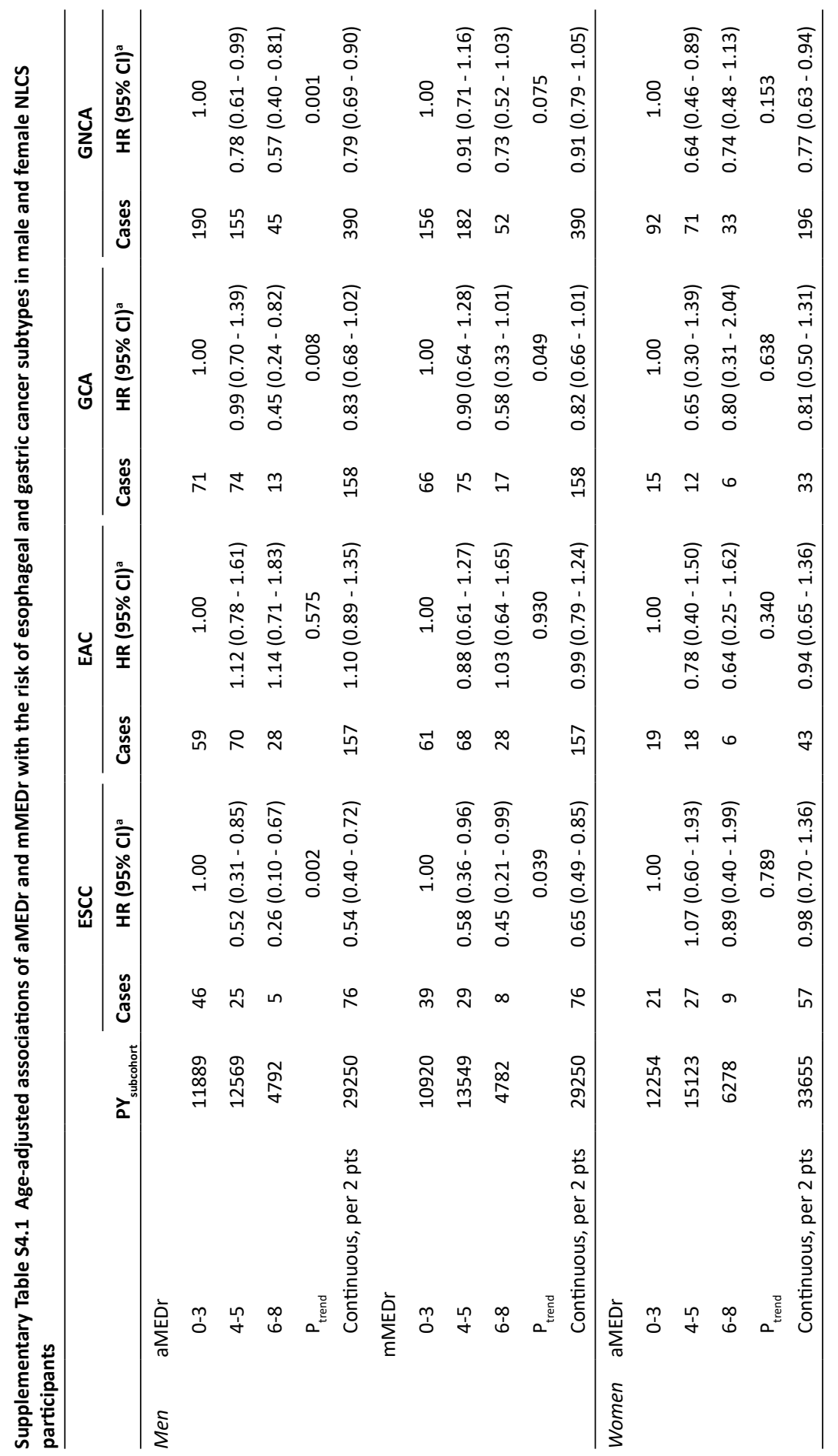




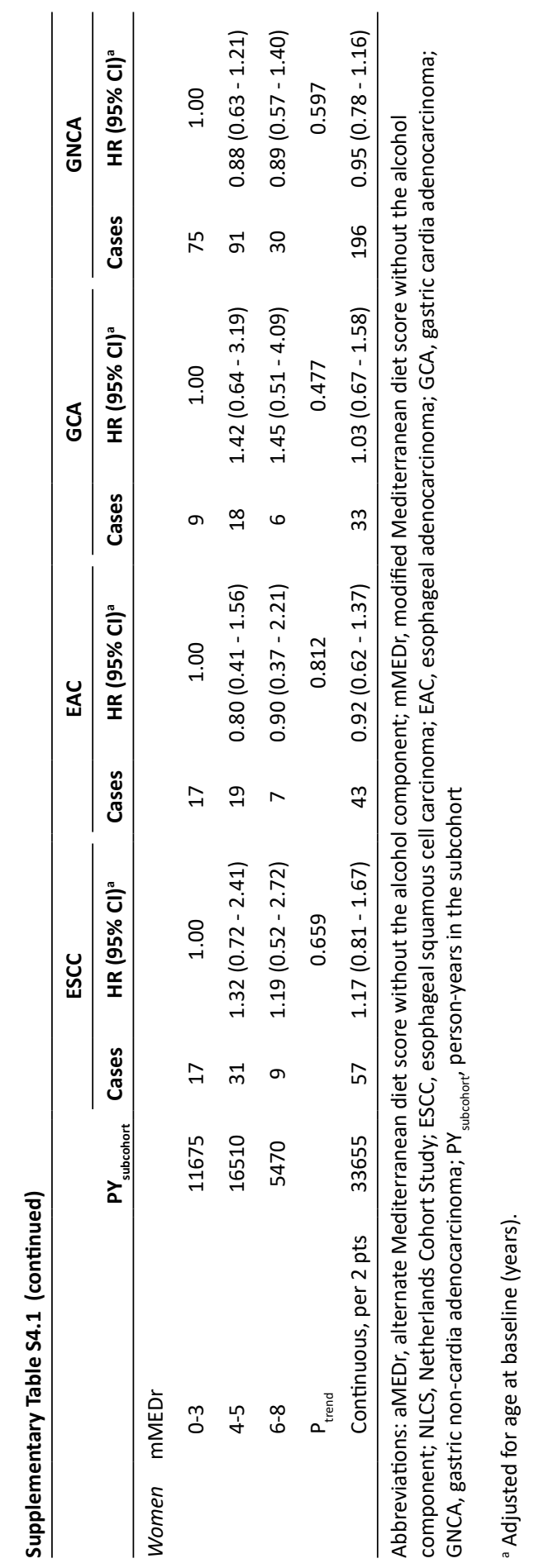


Mediterranean diet and risk of esophageal and gastric cancer 


\section{Chapter 5}

\section{Mediterranean diet adherence and risk of pancreatic cancer: A pooled analysis of two Dutch cohorts}

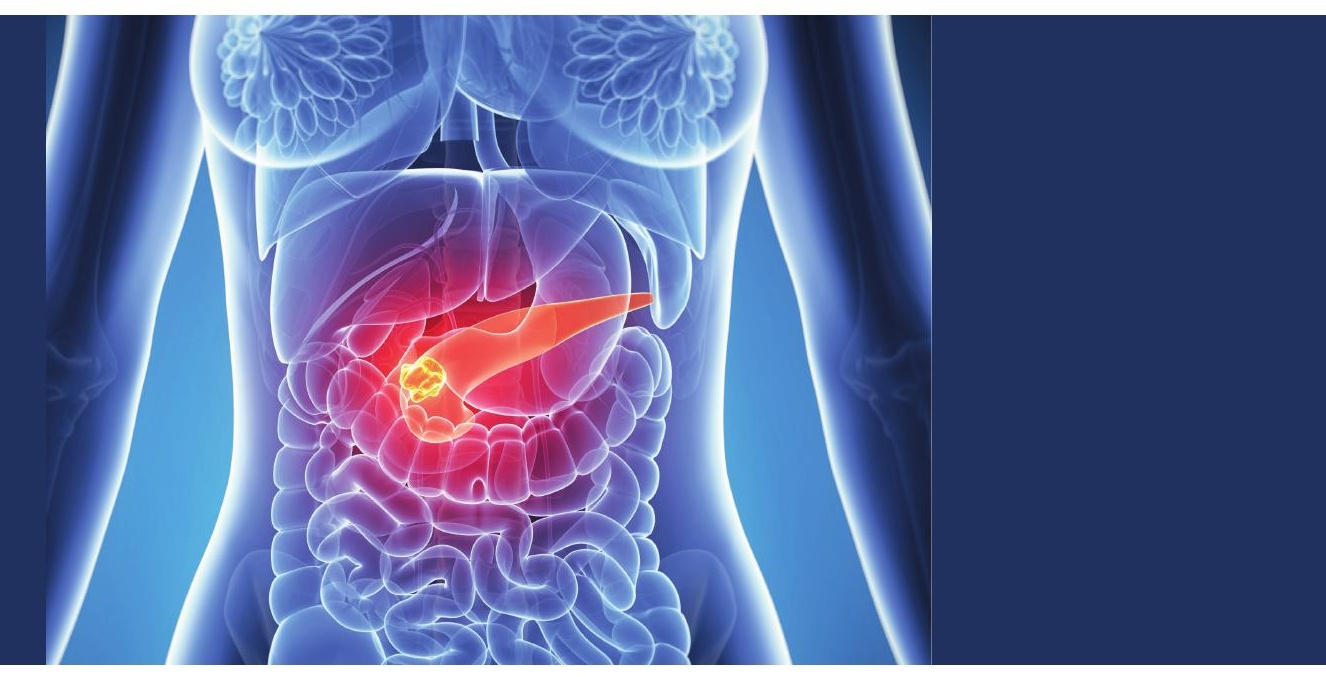

Maya Schulpen, Petra H. Peeters, Piet A. van den Brandt Int J Cancer. 2019; 144(7): 1550-1560 


\begin{abstract}
Studies investigating the association of Mediterranean diet (MD) adherence with pancreatic cancer risk are limited and had inconsistent results. We examined the association between MD adherence and pancreatic cancer incidence by pooling data from the Netherlands Cohort Study (NLCS, 120852 subjects) and the Dutch cohort of the European Prospective Investigation into Cancer and Nutrition (EPIC-NL, 40011 subjects). MD adherence was assessed using alternate and modified Mediterranean diet scores (aMED and mMED, respectively), including and excluding alcohol. After median follow-ups of 20.3 (NLCS) and 19.2 (EPIC-NL) years, 449 microscopically confirmed pancreatic cancer (MCPC) cases were included in study-specific multivariable Cox models. Study-specific estimates were pooled using a random-effects model. MD adherence was not significantly associated with MCPC risk in pooled and study-specific analyses, regardless of sex and MD score. Pooled hazard ratios (95\% confidence interval) for high (6-8) compared to low (0-3) values of mMED excluding alcohol were $0.66(0.40-1.10)$ in men and $0.94(0.63-1.40)$ in women. In never smokers, mMED excluding alcohol seemed to be inversely associated with MCPC risk (non-significant). However, no association was observed in ever smokers ( $p_{\text {heterogeneity }}=0.03$ ). Hazard ratios were consistent across strata of other potential effect modifiers. Considering MD scores excluding alcohol, mMED-containing models generally fitted better than aMEDcontaining models, particularly in men. Although associations somewhat differed when all pancreatic cancers were considered instead of MCPC, the overall conclusion was similar. In conclusion, MD adherence was not associated with pancreatic cancer risk in a pooled analysis of two Dutch cohorts.
\end{abstract}




\section{Introduction}

Despite its relatively low incidence, pancreatic cancer was ranked as the third most common cause of cancer death in the US based on 2010-2014 data [1]. Because the early disease stages are usually asymptomatic, pancreatic cancer is generally diagnosed in advanced disease stages resulting in a poor prognosis; five-year survival rates of pancreatic cancer in the US (2007-2013) were only $8.2 \%$ for all stages and $2.7 \%$ for distant stages [2, 3]. Diet could be a modifiable target for the primary prevention of pancreatic cancer. However, the World Cancer Research Fund/American Institute for Cancer Research (WCRF/AICR) judged in their 2012 report on pancreatic cancer that the evidence supporting an association between dietary factors and pancreatic cancer is limited [2]. Only body fatness (convincing evidence) and greater childhood growth (probable evidence) were reported to be associated with an increased pancreatic cancer risk [2].

The plant-based traditional Mediterranean dietary pattern (MD) is characterized by a high intake of vegetables, legumes, fruits, nuts, whole grains, olive oil (rich in monounsaturated fatty acids (MUFA)) and fish. In contrast, high-fat dairy products, red and processed meats, refined grains and sweets are consumed in small amounts. Alcohol consumption is considered moderate in the MD [4-6]. High adherence to the MD has been shown to reduce cardiovascular disease incidence and mortality, as well as all-cause mortality [7-9]. Recently, researchers have taken an increasing interest in the potentially beneficial effect of MD adherence on cancer risk [10].

Up until now, the association between a priori defined MD adherence and the incidence of pancreatic cancer has been investigated in three studies (1 case-control, 2 prospective cohorts), with inconsistent results [11-13]. An Italian hospital-based casecontrol study showed a statistically significantly decreased risk of pancreatic cancer with higher MD adherence [12]. On the other hand, the reduced pancreatic cancer risk associated with higher MD adherence was not significant in a US prospective cohort study [11] and there was no evidence of an association in the European Prospective Investigation into Cancer and Nutrition (EPIC) cohort [13]. In addition to the results concerning pancreatic cancer incidence, a significant decrease in pancreatic cancer mortality was associated with higher MD adherence in Swedish subjects [14]. It has previously been shown that associations of factors with pancreatic cancer risk depend on the microscopic confirmation status of the cases $[15,16]$. The most valid results are obtained by restricting analyses to microscopically confirmed pancreatic cancer (MCPC) cases, which minimizes misclassification of disease status $[15,16]$. Results for this subgroup of cases have only been reported in EPIC before [13].

The aim of the present analysis was to prospectively investigate the association of MD adherence with pancreatic cancer risk, using various a priori defined MD scores with and without alcohol. Analyses were performed considering all pancreatic cancer cases and MCPC cases specifically. We investigated these aims by pooling results of the Netherlands Cohort Study (NLCS) and the Dutch EPIC cohort (EPIC-NL) to increase the statistical power. 


\section{Materials and methods}

\section{Study population and pancreatic cancer follow-up}

A pooled analysis was conducted including individual participant data from the NLCS and EPICNL cohorts. Detailed descriptions of both cohorts have been published previously [17-23]. In short, the NLCS is a nationwide population-based cohort study among 58279 men and 62573 women from 204 Dutch municipalities, who were aged between 55 and 69 years at enrollment [17]. At baseline in September 1986, participants completed a self-administered questionnaire on diet and other cancer risk factors. For efficiency, data were processed and analyzed using the nested case-cohort design. Therefore, cases were derived from the entire NLCS cohort, whereas the number of person-years at risk was estimated based on a subcohort $(N=5000)$. Subcohort members were randomly sampled immediately after baseline and were followed up biennially for vital status information using municipal population registries [17, 20, 24]. The EPIC-NL cohort comprises 40011 subjects, who were included in the EPIC-Prospect (17357 women, aged 49-70 years) or EPIC-MORGEN (10260 men and 12394 women, aged 20-65 years) cohorts [21-23]. Cohort members of EPIC-Prospect were participants of a breast cancer screening program in the region of Utrecht between 1993 and 1997, whereas EPIC-MORGEN was composed by selecting random population samples of three Dutch towns (Amsterdam, Maastricht and Doetinchem) in the same time period. Baseline measurements were performed using a general questionnaire and a food frequency questionnaire (FFQ). In addition, physical examinations, including measurements of height, weight and blood pressure, were carried out at baseline. Vital status information of EPICNL participants was retrieved via linkage with the municipal population registries [21-23]. The NLCS and EPIC-NL cohorts were approved by the internal review boards of the institutions involved. All study participants consented to participation by completing the questionnaire (NLCS) or signing an informed consent form (EPIC-NL).

Incident cases of pancreatic cancer (International Classification of Diseases for Oncology, Third Edition (ICD-O-3), code C25) were identified by annual record linkage with the Netherlands Cancer Registry and PALGA, the nationwide Dutch Pathology Registry [18, 23]. Cases with endocrine pancreatic cancer, defined by ICD-O-3 code C25.4 or an endocrine tumor type, were excluded and censored at their date of diagnosis. Pancreatic cancer cases were considered to have MCPC, when they were diagnosed based on hematological, cytological or histological confirmation. Subjects were excluded from the analyses if they met one of the following criteria: prevalent cancer at baseline, except non-melanoma skin cancer (EPIC-NL) or any type of skin cancer (NLCS), or missing; incomplete, inconsistent or missing (dietary) questionnaires; a ratio of energy intake to basal metabolic rate in the lowest or highest $0.5 \%$ (EPIC-NL only); or incomplete data on alcohol consumption and variables necessary to calculate MD adherence. In total, 4084 of the NLCS subcohort members were eligible for inclusion in the analyses. In the EPIC-NL cohort, 35459 subjects met the eligibility criteria. Using 20.3 years of follow-up, 651 cases of exocrine pancreatic cancer (391 MCPC) were diagnosed in the NLCS. In the EPIC-NL cohort, 142 cases (104 MCPC) were detected during a median follow-up of 19.2 years. Observations were censored at December $31^{\text {st }}$ 2006 (NLCS) and December $31^{\text {st }} 2014$ (EPIC-NL). The selection process of subjects eligible for inclusion in the analyses is visualized in the flow diagrams in Supplementary Figure S5.1 (NLCS) and Supplementary Figure S5.2 (EPIC-NL). 


\section{Exposure assessment}

The habitual dietary intake over the year preceding enrollment was assessed by studyspecific, self-administered, semi-quantitative FFQs, of which the validity and reproducibility have been evaluated [19, 25-27]. Dutch food composition (NEVO) tables from the years 1986 (NLCS) and 1998 (EPIC-NL) were utilized to calculate mean daily nutrient intakes [28].

\section{Mediterranean diet adherence}

Relative MD adherence was assessed using the alternate and modified Mediterranean diet scores (aMED and $\mathrm{mMED}$, respectively), which are two variants of the original traditional Mediterranean diet score (tMED) developed by Trichopoulou et al. [29-33]. Before calculation of the MD scores, food intakes were adjusted to total energy intakes of 2000 (women) and 2500 (men) kilocalories (kcal) per day to correct for differences in daily energy intakes [29,33]. aMED is calculated based on the daily intakes of nine dietary components, which are each scored by 0 or 1 points, resulting in a sum score ranging from 0 (minimal $\mathrm{MD}$ adherence) to 9 (maximal MD adherence) [32,33]. A score of 1 is assigned to: high intakes ( $\geq$ sex-specific median) of vegetables (excluding potatoes), legumes, fruits, nuts, whole grains, and fish; a high ( $\geq$ sex-specific median) ratio of MUFA to saturated fatty acids (SFA); a low intake (< sex-specific median) of red and processed meats; and a moderate alcohol intake (5-25 grams per day (g/day) for both sexes) [32,33]. aMED is calculated in a similar way as tMED, but differs from the original score with respect to the composition of the dietary components. In tMED $[29,30]$, fruits and nuts are combined, total intakes of cereals and meats are considered, and consumption of dairy products is included (1 point if < sex-specific median). Besides, moderate alcohol consumption is defined differently in men (10-50 g/day) and women (5-25 g/day) [29, 30]. mMED [31] was specifically developed for usage in non-Mediterranean populations and differs from tMED with respect to the fatty acid ratio included. In MMED, the ratio of unsaturated fatty acids (polyunsaturated fatty acids (PUFA) + MUFA) to SFA replaces the MUFA:SFA ratio [31]. Sex-specific median intakes of dietary components were calculated separately for the NLCS and EPIC-NL cohorts. We also created reduced variants of aMED and mMED without alcohol (aMEDr and mMEDr, respectively), because moderate/heavy alcohol consumption ( $>3$ drinks per day) might increase pancreatic cancer risk [2,34]. aMEDr and mMEDr ranged from 0 to 8 points. Based on their MD score, subjects were categorized as having low (0-3), middle (4-5), or high (6$8(9))$ levels of MD adherence [31,33]. Additionally, MD scores were included as continuous terms to obtain effect estimates per two-point increment in score.

\section{Statistical analyses}

All analyses were performed separately for men and women unless otherwise specified. As a general approach, we first determined study-specific (NLCS and EPIC-NL) estimates, which were pooled in a later stage.

Cox proportional hazards models with follow-up as time variable were run to estimate studyspecific hazard ratios (HRs) and $95 \%$ confidence intervals $(95 \% \mathrm{Cls})$ for the relation between $\mathrm{MD}$ adherence and pancreatic cancer incidence. (Sub)cohort members were considered to be at risk from baseline until pancreatic cancer diagnosis, death, emigration, loss to followup or end of follow-up, whichever came first. Since the case-cohort design introduces additional variance, the Huber-White sandwich estimator was used to estimate standard 
errors of the HRs in the NLCS cohort [35]. Scaled Schoenfeld residuals tests and $-\operatorname{In}(-\operatorname{In})$ survival plots were used to evaluate the proportional hazards (PH) assumption [36]. In case of potential violations of the $\mathrm{PH}$ assumption for covariates, it was checked whether inclusion of a time-varying covariate altered the effect estimates of the exposures of interest.

aMEDr and mMEDr were modelled as both categorical and continuous variables. In order to perform tests for trends across the MD adherence categories, study- and sexspecific median values among (sub)cohort members were assigned to the MD adherence categories. Next, the created variable was fitted as a continuous term in the Cox model and statistical significance of the regression coefficient was assessed by the Wald test. Based on the literature, the following potential confounders were included in multivariable Cox models: age at baseline, sex (except for sex-specific analyses), cigarette smoking status, cigarette smoking frequency, cigarette smoking duration, body mass index (BMI), total daily energy intake, alcohol consumption (except for models containing the original MD scores including alcohol), history of (type 2) diabetes, level of education, and (non-occupational) physical activity. Cigarette smoking frequency and duration were combined into pack-years of smoking in the EPIC-NL cohort. Models based on NLCS data were additionally adjusted for family history of pancreatic cancer (not available for EPIC-NL), whereas the EPIC-NL models were also adjusted for cohort (EPIC-Prospect or EPIC-MORGEN).

Study-specific HRs were combined using a DerSimonian and Laird random-effects model to obtain pooled effect estimates for the association between MD adherence and pancreatic cancer risk. Weights were assigned to the study-specific estimates based on the inverse of their variances $[37,38]$.

The fits of aMEDr- and mMEDr-containing models (study-specific) were compared using Akaike's Information Criterion (AIC) [39]. In addition, AIC was used to compare performances of study-specific models containing MD score variants with and without alcohol. To evaluate whether the relation between MD adherence and pancreatic cancer risk is influenced by the microscopic confirmation status of the cases, we also restricted the analyses to MCPC cases. This article will mainly focus on results obtained among MCPC cases, as the most valid results are obtained in this case group $[15,16]$. Because moderate/heavy alcohol consumption might increase the risk of pancreatic cancer, we give priority to the use of MD scores without alcohol $[2,34]$.

Furthermore, it was evaluated whether the relation between MD adherence and pancreatic cancer risk varied across strata of potential effect modifiers. HRs for strata of cigarette smoking status, BMI, alcohol consumption and history of diabetes were retrieved by pooling study-specific effect estimates. Similarly, pooled regression coefficients were obtained for interaction terms between the MD scores and the potential effect modifiers and significance of the interactions was tested. Finally, because preclinical disease could potentially alter a subject's diet and therefore influence the observed association between MD adherence and pancreatic cancer risk, sensitivity analyses excluding the first two years of follow-up were performed on the individual study level. Statistical analyses were performed using Stata15 (StataCorp LLC, College Station, TX, USA). All presented p-values are two-sided. Statistical significance was defined as a $\mathrm{p}$-value below 0.05 . 


\section{Results}

After median follow-up times of 20.3 (NLCS) and 19.2 (EPIC-NL) years, 793 (men: 378; women: 415 ) eligible cases of exocrine pancreatic cancer were diagnosed in the total study population of whom 495 (men: 245; women: 250) were microscopically confirmed.

Study-specific baseline characteristics of the included cohorts are presented in Table 5.1 and Table 5.2. As expected, mean MD score values in the cohorts were similar. In contrast, noteworthy age differences were observed between the cohorts, with a substantially higher median age in the NLCS. Within the EPIC-NL cohort, women were older than men. Compared to EPIC-NL participants, NLCS participants were less often current smokers (women only), lower educated, less physically active, had lower daily intakes of energy and alcohol, had a lower BMI and were more likely to have a history of diabetes. The described differences between the cohorts might (partly) be attributed to variations in age and other study characteristics, such as participant recruitment criteria, time period of study, measurement methods and variable definitions.

As is shown in Table 5.1 and Table 5.2, mean MD score values were similar for MCPC cases and (sub)cohort members, except for aMEDr in EPIC-NL. Compared to (sub)cohort members, MCPC cases were older (EPIC-NL only), consumed more alcohol, had a higher BMI and were more likely to have a history of diabetes. MCPC cases were also more often current smokers. However, this did not apply for female MCPC cases in EPIC-NL, who were less often current smokers.

Table 5.3 and Table 5.4 present pooled and study-specific results of the multivariable Cox proportional hazards analyses evaluating sex-specific associations of MD adherence, measured by various MD scores, with MCPC risk. Due to missing values in covariates, 46 (9.3\%) MCPC cases (NLCS: 43 (11.0\%); EPIC-NL: 3 (2.9\%)) and 1307 (3.3\%) (sub)cohort members (NLCS: 364 (8.9\%); EPIC-NL: $943(2.7 \%)$ ) could not be included in the multivariable Cox proportional hazards analyses.

MD adherence was not statistically significantly associated with MCPC risk among men in the pooled multivariable analyses (Table 5.3). Pooled HRs $(95 \% \mathrm{Cl})$ comparing high to low MD adherence were $0.70(0.44-1.12)$ and $0.66(0.40-1.10)$ for aMEDr and mMEDr, respectively. Although the HR was not significant, middle mMEDr values seemed to be associated with an increased risk of MCPC. There was also no evidence of an association between $\mathrm{MD}$ adherence and $\mathrm{MCPC}$ risk when HRs were estimated per two-point increment in $\mathrm{MD}$ score (aMEDr=0.96, 95\% Cl: $0.80-1.16$; mMEDr=0.99, 95\% Cl: $0.83-1.18$ ). The observed associations were consistent among the individual cohorts (Table 5.3). The - In(In) survival plots indicated a potential violation of the $\mathrm{PH}$ assumption for the MD scores in men in the EPIC-NL cohort. However, PH assumption tests were not statistically significant. Furthermore, results generally similar to those for MCPC were obtained when all pancreatic cancer cases were included in the analyses (Supplementary Table S5.1).

As in men, we observed no association between MD adherence and MCPC risk among women in the pooled multivariable analyses (Table 5.4). Pooled HRs ( $95 \% \mathrm{CI}$ ) per two-point increment in score were $1.07(0.89-1.27)$ and $1.01(0.84-1.21)$ for aMEDr and mMEDr, respectively. Associations seemed to differ between the individual cohorts, particularly when MD adherence was expressed using mMEDr categories. Overall, mMEDr was not associated with MCPC risk in the NLCS, though a non-significantly reduced MCPC risk seemed to be associated with middle mMEDr values $\left(\mathrm{HR}_{\text {middle vs. low }}=0.71,95 \% \mathrm{Cl}: 0.49-1.01\right)$. In contrast 


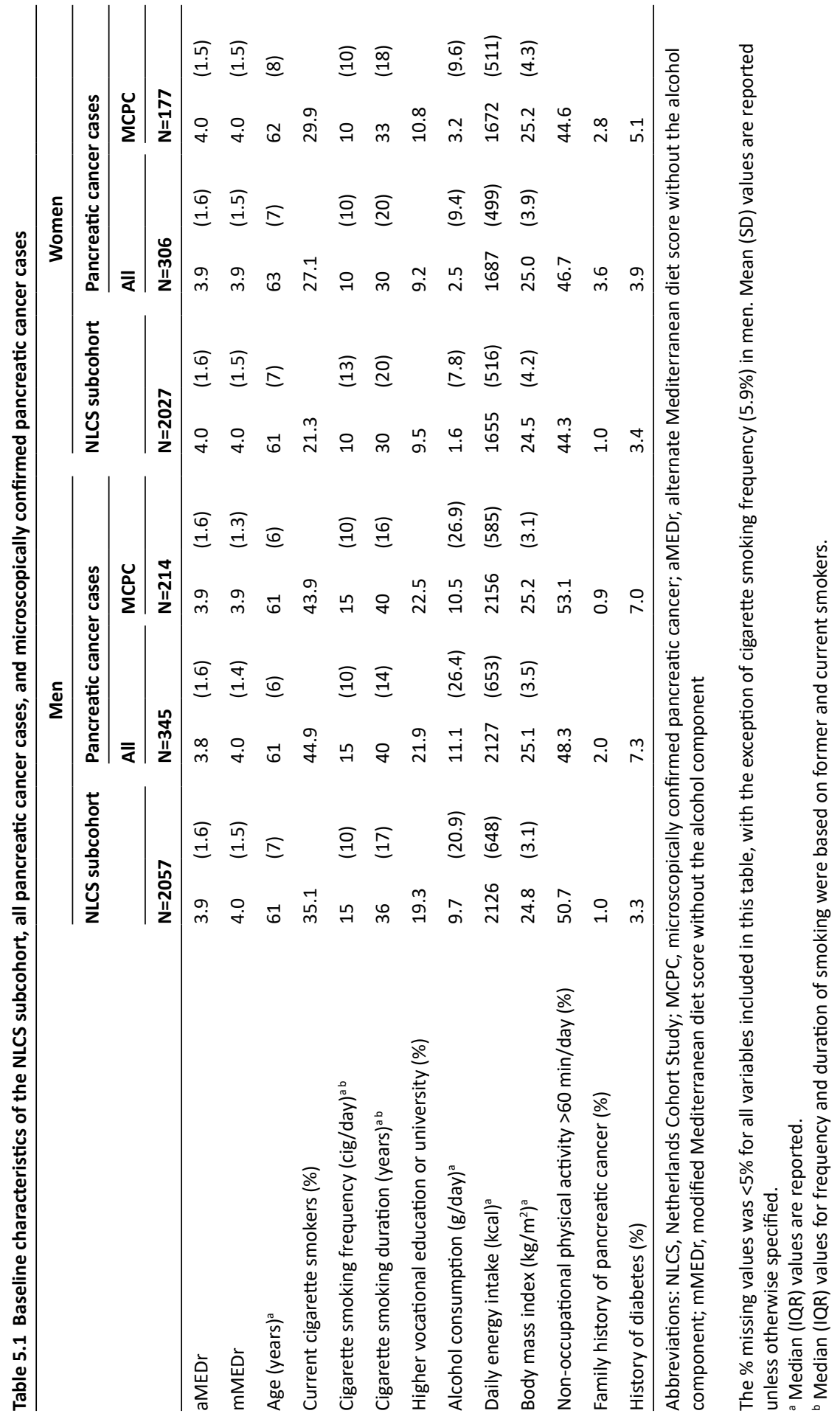




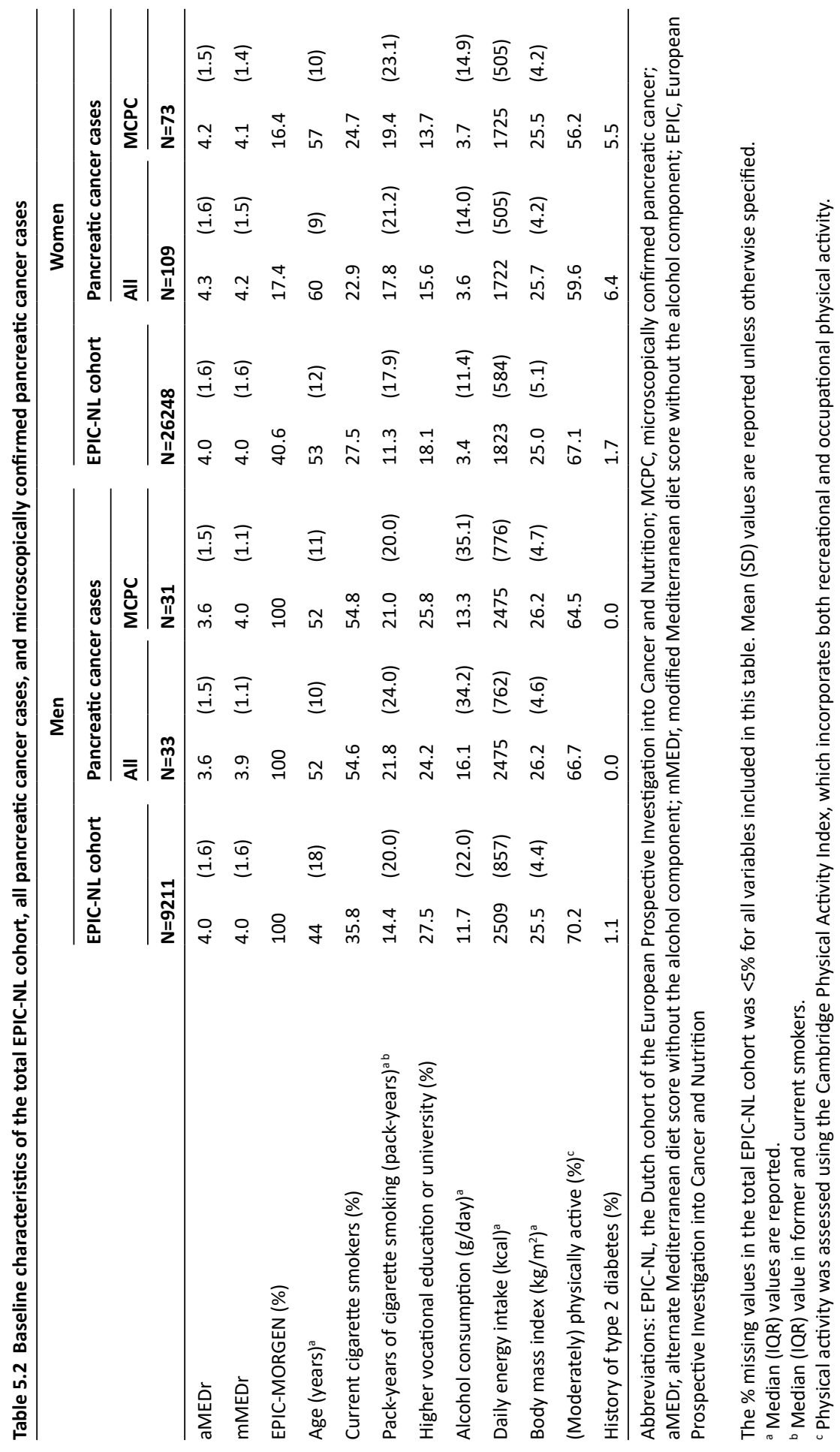




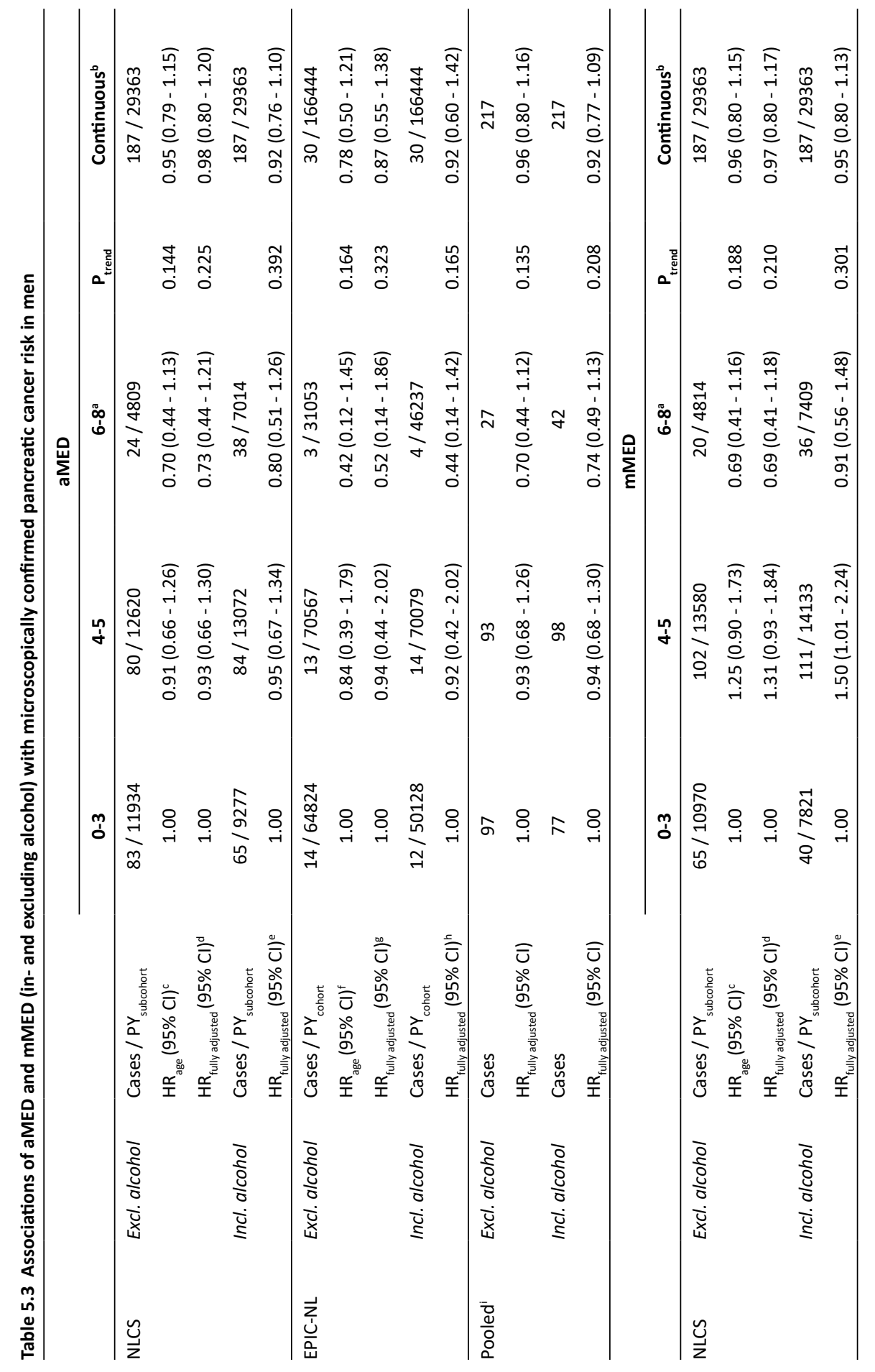




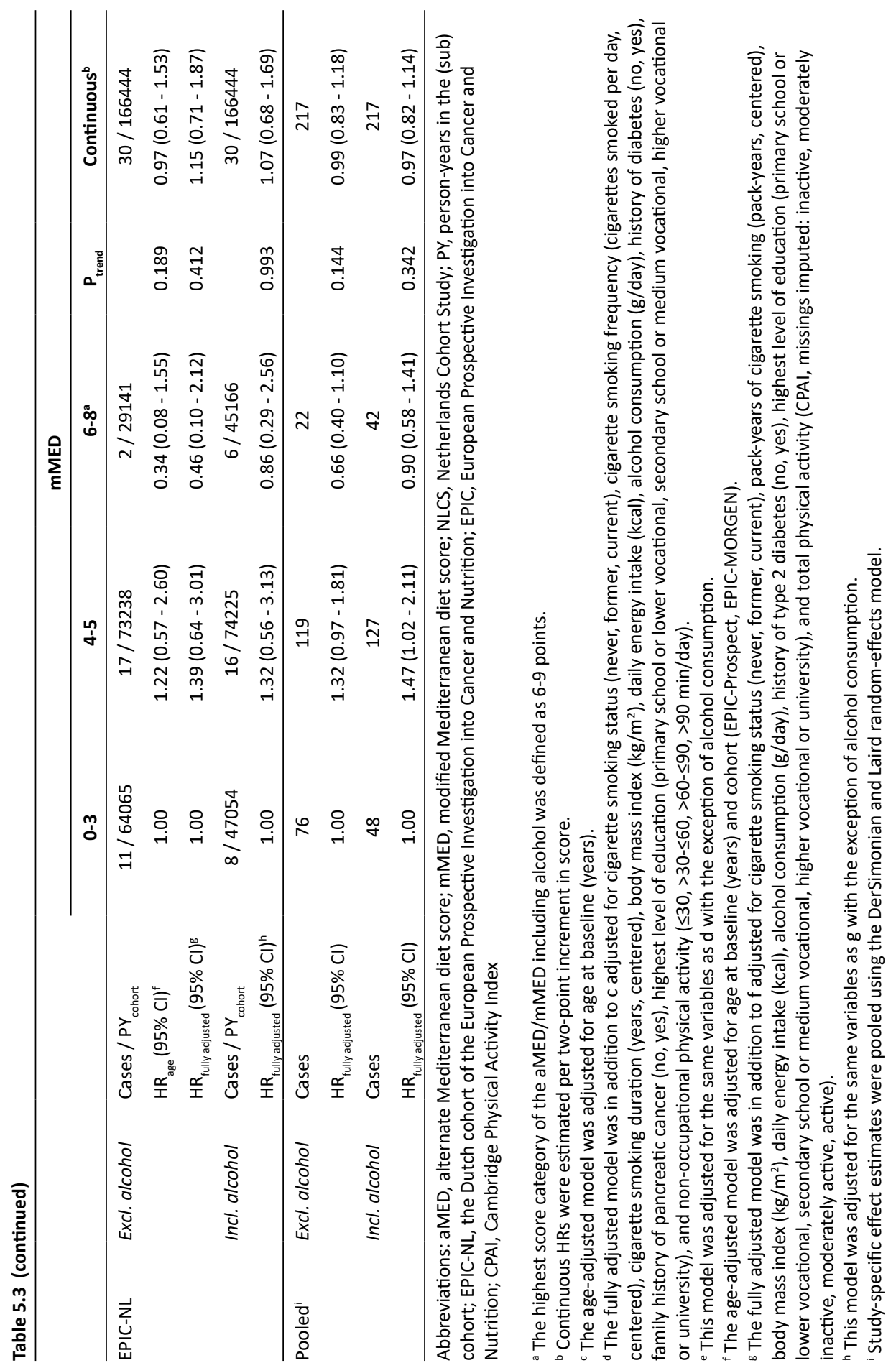




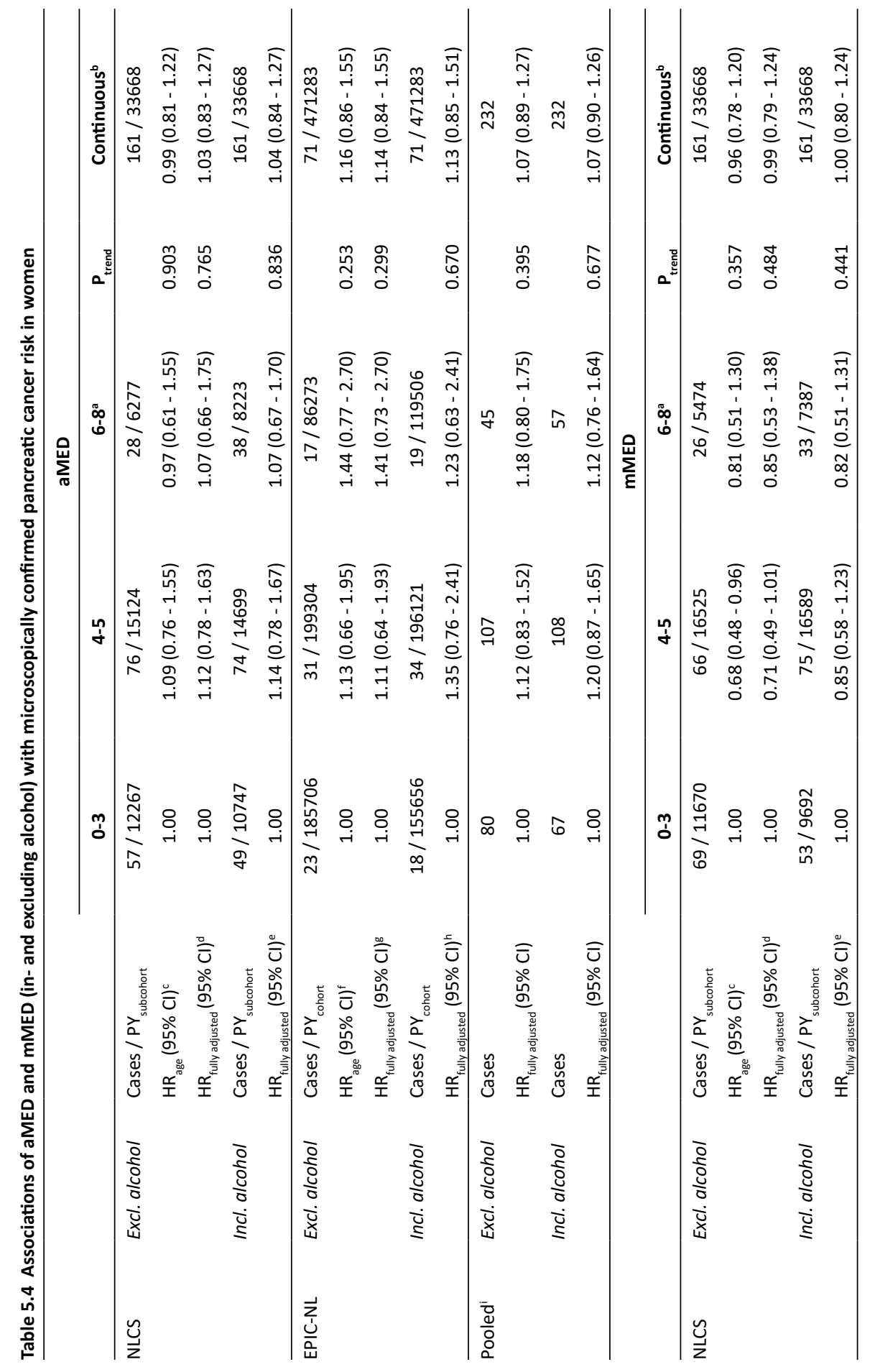




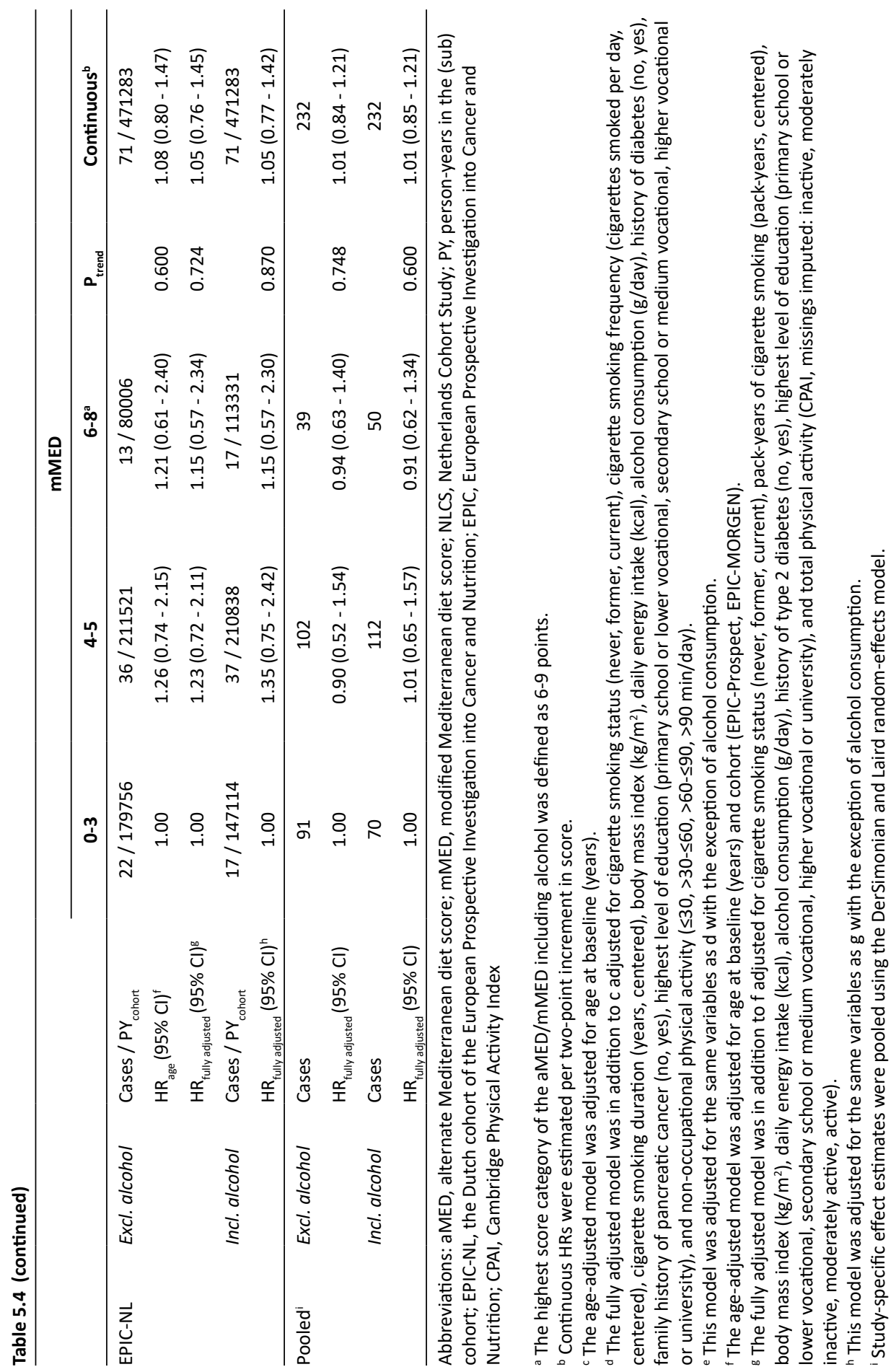


to the NLCS findings, associations between the categorical mMEDr and MCPC risk were absent or slightly positive in EPIC-NL. There was also no evidence of an association between $\mathrm{MD}$ adherence and pancreatic cancer risk in women when all pancreatic cancer cases were included in the analyses (Supplementary Table S5.2).

Study-specific tests for heterogeneity between the sexes were mostly not statistically significant, except when MD adherence was assessed by mMEDr categories in the NLCS. Comparing study-specific AIC values, we found that mMEDr-containing models generally fitted better than models containing aMEDr, particularly in men. In women, this pattern was unclear. Also, no consistent pattern was observed when performances of models containing MD scores with and without alcohol were compared.

Table 5.5 shows pooled associations between MD adherence and the risk of MCPC within strata of potential effect modifiers. Since study-specific tests for heterogeneity between the sexes were not statistically significant when MD adherence was modelled using continuous MD scores, study-specific subgroup results based on both sexes were pooled to increase the statistical power. The association between mMEDr and MCPC risk differed statistically significantly across the strata of cigarette smoking status $\left(p_{\text {heterogeneity }}=0.03\right)$. Although not significant, mMEDr seemed to be inversely associated with MCPC risk in never smokers, whereas there was no evidence of an inverse association in ever smokers. A similar, but weaker and non-significant, pattern was noticed when MD adherence was assessed using aMEDr. No differences in associations were observed across strata of BMI, alcohol consumption and history of diabetes. Finally, exclusion of the first two years of follow-up did not alter the study-specific results. However, in the NLCS, we did note a non-significant inverse association between MD adherence and MCPC risk when focusing on the first two years of follow-up. In EPIC-NL, too few cases were available to perform this analysis.

\section{Discussion}

MD adherence was not significantly associated with MCPC risk in pooled multivariable analyses, including NLCS and EPIC-NL data, as well as study-specific multivariable analyses, regardless of sex and MD score used. The model fit was generally better for mMEDrcontaining models compared to aMEDr-containing models, especially in men. Comparison of performances of models containing MD scores with and without alcohol did not show a consistent pattern. Stratified analyses indicated an inverse association between mMEDr and MCPC risk in never smokers (non-significant), but not in ever smokers ( $p_{\text {heterogeneity }}=0.03$ ). There was no evidence for effect modification by BMI, alcohol consumption or history of diabetes.

Results of our pooled analysis are in line with those obtained in previous prospective cohort studies $[11,13]$ that did also not find a statistically significant inverse association between MD adherence and pancreatic cancer incidence. In diabetes-free participants of the National Institutes of Health-American Association of Retired Persons (NIH-AARP) Diet and Health Study, a HR of 0.92 (95\% Cl: $0.81-1.05)$ was observed when comparing high (aMEDr: 5-8) to low (0-4) MD adherence [11]. In the same study, a non-significantly reduced pancreatic cancer risk was found when scores of 7-8 were compared to scores of $0-1(p=0.06)$. Furthermore, there was no indication for an inverse association between MD adherence, assessed by a variant of the relative Mediterranean diet score excluding alcohol, and pancreatic cancer risk in the EPIC cohort $\left(\mathrm{HR}_{\text {high vs. low }}=0.99,95 \% \mathrm{Cl}: 0.77-1.26\right)$ [13]. 
Table 5.5 Pooled results for fully adjusted associations ${ }^{\mathrm{a}}$ of aMEDr and mMEDr with microscopically confirmed pancreatic cancer risk for various subgroups

\begin{tabular}{|c|c|c|c|c|c|c|c|}
\hline \multirow[b]{2}{*}{ Subgroup } & \multirow[b]{2}{*}{ Cases } & \multicolumn{3}{|c|}{ aMEDr (per two-point increment) } & \multicolumn{3}{|c|}{ mMEDr (per two-point increment) } \\
\hline & & $\mathrm{HR}_{\text {pooled }}{ }^{b}$ & $(95 \% \mathrm{Cl})$ & $\mathbf{P}_{\text {heterogeneity }}{ }^{\mathrm{c}}$ & $\mathrm{HR}_{\text {pooled }}{ }^{\mathrm{b}}$ & $(95 \% \mathrm{Cl})$ & $\mathbf{P}_{\text {heterogeneity }}{ }^{\mathbf{c}}$ \\
\hline Overall & 449 & 1.03 & $(0.90-1.16)$ & & 1.01 & $(0.89-1.15)$ & \\
\hline \multicolumn{8}{|l|}{ Cigarette smoking status ${ }^{d}$} \\
\hline Never smokers & 137 & 0.89 & $(0.71-1.13)$ & & 0.81 & $(0.65-1.02)$ & \\
\hline Ever smokers & 312 & 1.08 & $(0.93-1.25)$ & 0.230 & 1.11 & $(0.94-1.31)$ & 0.034 \\
\hline \multicolumn{8}{|l|}{ Body mass index } \\
\hline$\geq 18.5-<25.0 \mathrm{~kg} / \mathrm{m}^{2}$ & 203 & 1.03 & $(0.85-1.25)$ & & 1.01 & $(0.84-1.21)$ & \\
\hline$\geq 25.0 \mathrm{~kg} / \mathrm{m}^{2}$ & 245 & 1.04 & $(0.87-1.24)$ & 0.742 & 1.03 & $(0.86-1.24)$ & 0.926 \\
\hline \multicolumn{8}{|l|}{ Alcohol consumption ${ }^{\mathrm{g} g}$} \\
\hline$>0-<15.0 \mathrm{~g} /$ day & 244 & 1.09 & $(0.92-1.28)$ & & 1.07 & $(0.90-1.26)$ & \\
\hline$\geq 15.0 \mathrm{~g} /$ day & 139 & 0.89 & $(0.69-1.14)$ & 0.215 & 0.91 & $(0.72-1.15)$ & 0.264 \\
\hline \multicolumn{8}{|l|}{ History of diabetes ${ }^{h}$} \\
\hline No & 423 & 1.02 & $(0.89-1.16)$ & & 1.02 & $(0.89-1.16)$ & \\
\hline Yes & 26 & 1.48 & $(0.62-3.49)$ & 0.634 & 0.80 & $(0.37-1.74)$ & 0.672 \\
\hline
\end{tabular}

Abbreviations: aMEDr, alternate Mediterranean diet score without the alcohol component; mMEDr, modified Mediterranean diet score without the alcohol component; NLCS, Netherlands Cohort Study; EPIC-NL, the Dutch cohort of the European Prospective Investigation into Cancer and Nutrition; EPIC, European Prospective Investigation into Cancer and Nutrition; CPAI, Cambridge Physical Activity Index

a The fully adjusted analyses in the NLCS cohort were adjusted for age at baseline (years), sex (men, women), cigarette smoking status (never, former, current), cigarette smoking frequency (cigarettes smoked per day, centered), cigarette smoking duration (years, centered), body mass index $\left(\mathrm{kg} / \mathrm{m}^{2}\right)$, daily energy intake (kcal), alcohol consumption (g/day), history of diabetes (no, yes), family history of pancreatic cancer (no, yes), highest level of education (primary school or lower vocational, secondary school or medium vocational, higher vocational or university), and non-occupational physical activity $(\leq 30,>30-\leq 60,>60-\leq 90,>90 \mathrm{~min} /$ day).

The fully adjusted analyses in the EPIC-NL cohort were adjusted for age at baseline (years), sex (men, women), cohort (EPIC-Prospect, EPIC-MORGEN), cigarette smoking status (never, former, current), pack-years of cigarette smoking (pack-years, centered), body mass index $\left(\mathrm{kg} / \mathrm{m}^{2}\right)$, daily energy intake (kcal), alcohol consumption (g/day), history of type 2 diabetes (no, yes), highest level of education (primary school or lower vocational, secondary school or medium vocational, higher vocational or university), and total physical activity (CPAI, missings imputed: inactive, moderately inactive, moderately active, active).

${ }^{b}$ Study-specific effect estimates were pooled using the DerSimonian and Laird random-effects model.

c P-values for heterogeneity between subgroups were obtained by testing the statistical significance of pooled interaction terms between aMEDr/mMEDr and the potential effect modifiers.

${ }^{\mathrm{d}}$ Not adjusted for cigarette smoking status.

e Not adjusted for body mass index.

${ }^{f}$ No pooled HRs could be obtained for non-consumers of alcohol, because no microscopically confirmed cases of pancreatic cancer were diagnosed in this subgroup in the EPIC-NL cohort.

g Not adjusted for alcohol consumption.

${ }^{\mathrm{h}}$ Not adjusted for history of (type 2 ) diabetes. 
In contrast, an Italian hospital-based case-control study did show a statistically significant inverse association between MD adherence (revised tMED including alcohol) and pancreatic cancer incidence (odds ratio ${ }_{\geq 6 \text { vs. }<3}=0.48,95 \% \mathrm{Cl}: 0.35-0.67$ ) [12]. Additionally, higher MD adherence according to an adapted version of mMED including alcohol was associated with a significant decrease in pancreatic cancer mortality in the prospective Västerbotten Intervention Program with a HR of 0.82 (95\% Cl: $0.72-0.94)$ per one-point increment in score [14]. However, the results of the latter study were based on only 92 pancreatic cancer deaths and might have been distorted by selection bias, as excluded participants were characterized by a higher mortality risk.

The main results of the present study were based on analyses that were restricted to MCPC cases. Restricting the analyses to MCPC cases minimizes misclassification of disease status and therefore renders the most valid results $[15,16]$. Non-MCPC cases may present with different subtypes of pancreatic cancer or non-pancreatic cancer. This could affect the observed association when these subtypes or non-pancreatic cancers are differentially related to the exposure of interest compared with MCPC [16]. Although we observed some differences between associations determined among all pancreatic cancer cases and MCPC cases in the present analysis, the overall conclusion was similar for both case sets. Of the previously conducted studies concerning MD adherence and pancreatic cancer risk, only the EPIC study by Molina-Montes et al. [13] reported results in MCPC cases specifically and concluded that exclusion of non-MCPC cases did not alter the effect estimates.

Dietary habits may be influenced by the presence of preclinical disease, in particular in case of gastrointestinal cancers. Preclinical disease symptoms may result in reduced intakes of Mediterranean foods, such as vegetables, fruits and nuts, by cases. This could cause casecontrol studies to find a protective effect, whereas in fact there is no association. With regard to MD adherence and pancreatic cancer incidence, only the case-control study by Bosetti et al. [12] observed a statistically significant inverse association. Moreover, in the NLCS cohort, we noted that higher MD adherence was associated with a non-significantly reduced MCPC risk when we only included the first two years of follow-up, whereas there was no evidence for a relation in later follow-up periods. This indicates that indeed the presence of preclinical disease could cause us to find an inverse association in the absence of a true effect.

Study-specific tests for heterogeneity showed that the association of MD adherence with MCPC risk did not significantly differ between the sexes in the NLCS and EPIC-NL, except when MD adherence was assessed by mMEDr categories in the NLCS. Likewise, previously conducted studies on the topic did also not observe clear differences in associations between men and women [11-14]. In our pooled analysis, the association of mMEDr, but not aMEDr, with MCPC risk differed statistically significantly across the strata of smoking status. Higher mMEDr values seemed to be associated with a decreased MCPC risk in never smokers (non-significant), but not in ever smokers. In contrast, previous studies did not observe an interaction with smoking status [12-14]. Future studies should further investigate the potential effect modifying role of smoking status in the association between MD adherence and pancreatic cancer risk. HRs were consistent across strata of the other potential effect modifiers that we evaluated, including history of diabetes. Similarly, there was no evidence for an interaction with diabetes status in the study by Molina-Montes et al. [13]. In contrast, the significant inverse association between $M D$ adherence and pancreatic cancer risk observed in the study by Bosetti et al. [12] was restricted to non-diabetics ( $p_{\text {heterogeneity }}=0.01$ ).

Comparing various MD score variants, we observed that mMEDr-containing models 
performed better than aMEDr-containing models in men, whereas no clear pattern was present in women. In contrast, previous NLCS analyses concerning breast and lung cancer consistently showed a better performance for aMEDr-containing models [40, 41]. Because moderate/heavy alcohol consumption might be associated with an increased pancreatic cancer risk [2,34], we also compared model performances of MD score variants including and excluding alcohol component, which showed no consistent pattern. Previously conducted studies evaluating the effect of considering alcohol as MD score component, found similar HRs for MD score variants with and without alcohol $[12,13]$.

A major strength of our study is the pooling of data of two cohorts, which increased the statistical power. However, it should be noted that the relatively low number of male MCPC cases in the EPIC-NL cohort caused the pooled results for men to primarily reflect the associations observed in the NLCS cohort. Another strength with respect to the pooled analyses was the availability of individual participant data, which enabled us to standardize the statistical methods as well as the exposure, confounding and outcome variables, thereby minimizing between-study heterogeneity. Additionally, we had access to detailed dietary data retrieved via FFQs of which the validity and reproducibility have been evaluated [19, 25-27]. Finally, the prospective designs and long durations of follow-up were other strengths of the included cohorts.

A potential weakness of the MD scores used, particularly in non-Mediterranean countries such as the Netherlands, is the population-dependent assignment of scores. Therefore, even though diets of subjects with higher MD scores in our study population can be considered to be more Mediterranean compared to those of subjects with lower MD scores, high MD scores do not necessarily reflect close adherence to a true MD. However, MD adherence was also not significantly associated with a reduced pancreatic cancer risk in the southern European countries of the EPIC cohort [13]. Olive oil is the principal source of fat in the traditional MD $[4,5]$. However, tMED $[29,30]$ and many of its derivatives, including the MD scores that we used, do not incorporate olive oil consumption as a specific component. Instead, a fatty acid ratio is used to model the high levels of MUFA (mainly from olive oil) and low levels of SFA characteristic of the Greek MD [29]. The use of a fatty acid ratio to reflect the high olive oil intake in the traditional MD improves the usage of tMED and its derivatives in nonMediterranean countries in which the olive oil consumption is generally low, as was also the case in the Netherlands at the time of our baseline measurements. Another weakness of our analysis was the reliance on single baseline measurements for dietary habits and potential confounding factors. Hence, changes in diet and/or confounding factors during follow-up might have attenuated the associations. However, it has been shown that the reproducibility of the FFQs used was generally good [25-27]. The NLCS-FFQ had an average test-retest correlation of 0.66 for all nutrients. After five years, the correlation between the baseline and repeated measurement of the NLCS-FFQ had declined on average only 0.07 [25]. The FFQ used in the EPIC-NL cohort had a median 12-month reproducibility for food groups of 0.71 for men and 0.77 for women [26]. Finally, errors in the measurements of dietary habits and residual confounding by unmeasured factors cannot fully be excluded.

In conclusion, higher MD adherence was not associated with a decreased risk of pancreatic cancer in a pooled analysis of two Dutch cohorts. 


\section{References}

1. National Cancer Institute. SEER Cancer Stat Facts: Pancreas cancer. Bethesda, MD: National Cancer Institute; 2017 [cited 2017 June]. Available from: https://seer.cancer.gov/statfacts/html/ pancreas.html.

2. World Cancer Research Fund / American Institute for Cancer Research. Continuous update project report. Food, nutrition, physical activity, and the prevention of pancreatic cancer. 2012. Available from: http://www.dietandcancerreport.org.

3. Howlader N, Noone AM, Krapcho M, Miller D, Bishop K, Kosary CL, et al. SEER Cancer Statistics Review, 1975-2014. Bethesda, MD: National Cancer Institute; 2017 [cited 2017 June]. Available from: http://seer.cancer.gov/csr/1975_2014/, based on November 2016 SEER data submission, posted to the SEER web site, April 2017.

4. Willett WC, Sacks F, Trichopoulou A, Drescher G, Ferro-Luzzi A, Helsing E, et al. Mediterranean diet pyramid: a cultural model for healthy eating. Am J Clin Nutr. 1995;61(6 Suppl):1402S-6S.

5. Trichopoulou A, Lagiou P. Healthy traditional Mediterranean diet: an expression of culture, history, and lifestyle. Nutr Rev. 1997;55(11 Pt 1):383-9.

6. Fung TT, Rexrode KM, Mantzoros CS, Manson JE, Willett WC, Hu FB. Mediterranean diet and incidence of and mortality from coronary heart disease and stroke in women. Circulation. 2009;119(8):1093-100.

7. Martinez-Gonzalez MA, Bes-Rastrollo M. Dietary patterns, Mediterranean diet, and cardiovascular disease. Curr Opin Lipidol. 2014;25(1):20-6.

8. Sofi F, Macchi C, Abbate R, Gensini GF, Casini A. Mediterranean diet and health status: an updated meta-analysis and a proposal for a literature-based adherence score. Public Health Nutr. 2014;17(12):2769-82.

9. Grosso G, Marventano S, Yang J, Micek A, Pajak A, Scalfi L, et al. A comprehensive metaanalysis on evidence of Mediterranean diet and cardiovascular disease: Are individual components equal? Crit Rev Food Sci Nutr. 2017;57(15):3218-32.

10. Schwingshackl L, Schwedhelm C, Galbete C, Hoffmann G. Adherence to Mediterranean Diet and Risk of Cancer: An Updated Systematic Review and Meta-Analysis. Nutrients. 2017;9(10).

11. Jiao L, Mitrou PN, Reedy J, Graubard BI, Hollenbeck AR, Schatzkin A, et al. A combined healthy lifestyle score and risk of pancreatic cancer in a large cohort study. Arch Intern Med. 2009;169(8):76470.

12. Bosetti C, Turati F, Dal Pont A, Ferraroni M, Polesel J, Negri E, et al. The role of Mediterranean diet on the risk of pancreatic cancer. Br J Cancer. 2013;109(5):1360-6.

13. Molina-Montes E, Sanchez MJ, Buckland G, Bueno-de-Mesquita HB, Weiderpass E, Amiano $P$, et al. Mediterranean diet and risk of pancreatic cancer in the European Prospective Investigation into Cancer and Nutrition cohort. Br J Cancer. 2017;116(6):811-20.

14. Tognon G, Nilsson LM, Lissner L, Johansson I, Hallmans G, Lindahl B, et al. The Mediterranean diet score and mortality are inversely associated in adults living in the subarctic region. J Nutr. 2012;142(8):1547-53.

15. Silverman DT, Schiffman M, Devesa S. Diagnostic certainty in pancreatic cancer. J Clin Epidemiol. 1996;49(5):601-3.

16. Verhage BA, Schouten LJ, Goldbohm RA, van den Brandt PA. Anthropometry and pancreatic cancer risk: an illustration of the importance of microscopic verification. Cancer Epidemiol Biomarkers Prev. 2007;16(7):1449-54.

17. van den Brandt PA, Goldbohm RA, van 't Veer P, Volovics A, Hermus RJ, Sturmans F. A largescale prospective cohort study on diet and cancer in The Netherlands. J Clin Epidemiol. 1990;43(3):28595.

18. van den Brandt PA, Schouten LJ, Goldbohm RA, Dorant E, Hunen PM. Development of a record linkage protocol for use in the Dutch Cancer Registry for Epidemiological Research. Int J Epidemiol. 1990;19(3):553-8.

19. Goldbohm RA, van den Brandt PA, Brants HA, van 't Veer P, Al M, Sturmans F, et al. Validation of a dietary questionnaire used in a large-scale prospective cohort study on diet and cancer. Eur J Clin Nutr. 1994;48(4):253-65.

20. Volovics A, van den Brandt PA. Methods for the analyses of case-cohort studies. Biometrical 
J. 1997;39(2):195-214.

21. Boker LK, van Noord PA, van der Schouw YT, Koot NV, Bueno de Mesquita HB, Riboli E, et al. Prospect-EPIC Utrecht: study design and characteristics of the cohort population. European Prospective Investigation into Cancer and Nutrition. Eur J Epidemiol. 2001;17(11):1047-53.

22. Blokstra A, Smit HA, Bueno de Mesquita HB, Seidell JC, Verschuren WMM. Monitoring project on chronic disease risk factors (MORGEN-project) 1993-1997: prevalences and trends in lifestyle and risk, report no. 263200008. Bilthoven, the Netherlands: National Institute for Public Health and the Environment; 2005. Available from: https://www.rivm.nl/Documenten_en_publicaties/ Wetenschappelijk/Rapporten/2005/december/Monitoring_van_Risicofactoren_en_Gezondheid_in_ Nederland_MORGEN_project_1993_1997_Leefstijl_en_risicofactoren_prevalenties_en_trends (in Dutch).

23. Beulens JW, Monninkhof EM, Verschuren WM, van der Schouw YT, Smit J, Ocke MC, et al. Cohort profile: the EPIC-NL study. Int J Epidemiol. 2010;39(5):1170-8.

24. Prentice RL. A case-cohort design for epidemiologic cohort studies and disease prevention trials. Biometrika. 1986;73(1):1-11.

25. Goldbohm RA, van ' $t$ Veer $P$, van den Brandt PA, van ' $t$ Hof MA, Brants HA, Sturmans F, et al. Reproducibility of a food frequency questionnaire and stability of dietary habits determined from five annually repeated measurements. Eur J Clin Nutr. 1995;49(6):420-9.

26. Ocke MC, Bueno-de-Mesquita HB, Goddijn HE, Jansen A, Pols MA, van Staveren WA, et al. The Dutch EPIC food frequency questionnaire. I. Description of the questionnaire, and relative validity and reproducibility for food groups. Int J Epidemiol. 1997;26 Suppl 1:S37-48.

27. Ocke MC, Bueno-de-Mesquita HB, Pols MA, Smit HA, van Staveren WA, Kromhout D. The Dutch EPIC food frequency questionnaire. II. Relative validity and reproducibility for nutrients. Int J Epidemiol. 1997;26 Suppl 1:S49-58.

28. NEVO table. Dutch food composition table. The Hague, the Netherlands: Voorlichtingsbureau voor de Voeding.

29. Trichopoulou A, Kouris-Blazos A, Wahlqvist ML, Gnardellis C, Lagiou P, Polychronopoulos E, et al. Diet and overall survival in elderly people. BMJ. 1995;311(7018):1457-60.

30. Trichopoulou A, Costacou T, Bamia C, Trichopoulos D. Adherence to a Mediterranean diet and survival in a Greek population. N Engl J Med. 2003;348(26):2599-608.

31. Trichopoulou A, Orfanos P, Norat T, Bueno-de-Mesquita B, Ocke MC, Peeters PH, et al. Modified Mediterranean diet and survival: EPIC-elderly prospective cohort study. BMJ. 2005;330(7498):991.

32. Fung TT, McCullough ML, Newby PK, Manson JE, Meigs JB, Rifai N, et al. Diet-quality scores and plasma concentrations of markers of inflammation and endothelial dysfunction. Am J Clin Nutr. 2005;82(1):163-73.

33. Mitrou PN, Kipnis V, Thiebaut AC, Reedy J, Subar AF, Wirfalt E, et al. Mediterranean dietary pattern and prediction of all-cause mortality in a US population: results from the NIH-AARP Diet and Health Study. Arch Intern Med. 2007;167(22):2461-8.

34. Maisonneuve P, Lowenfels AB. Risk factors for pancreatic cancer: a summary review of metaanalytical studies. Int J Epidemiol. 2015;44(1):186-98.

35. Lin DY, Wei LJ. The Robust Inference for the Cox Proportional Hazards Model. J Am Stat Assoc. 1989;84(408):1074-8.

36. Grambsch PM, Therneau TM. Proportional Hazards Tests and Diagnostics Based on Weighted Residuals. Biometrika. 1994;81(3):515-26.

37. DerSimonian R, Laird N. Meta-analysis in clinical trials. Control Clin Trials. 1986;7(3):177-88.

38. Smith-Warner SA, Spiegelman D, Ritz J, Albanes D, Beeson WL, Bernstein L, et al. Methods for pooling results of epidemiologic studies: the Pooling Project of Prospective Studies of Diet and Cancer. Am J Epidemiol. 2006;163(11):1053-64.

39. Akaike H. A new look at the statistical model identification. IEEE Transactions on automatic control. 1974;AC-19(6):716-23.

40. van den Brandt PA, Schulpen M. Mediterranean diet adherence and risk of postmenopausal breast cancer: results of a cohort study and meta-analysis. Int J Cancer. 2017;140(10):2220-31.

41. Schulpen $M$, van den Brandt PA. Adherence to the Mediterranean diet and risk of lung cancer in the Netherlands Cohort Study. Br J Nutr. 2018;119(6):674-84. 
Chapter 5

Supplementary materials

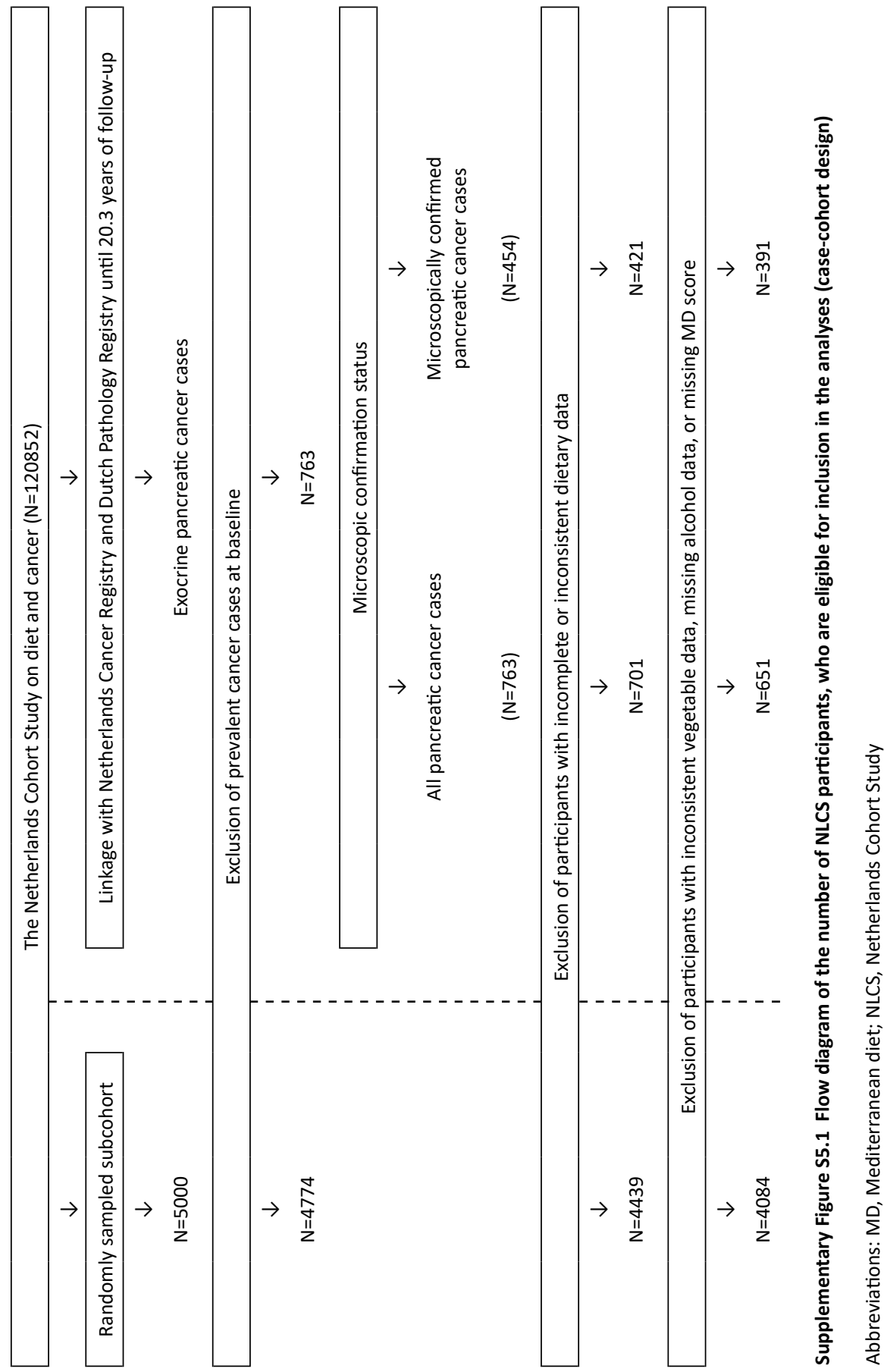




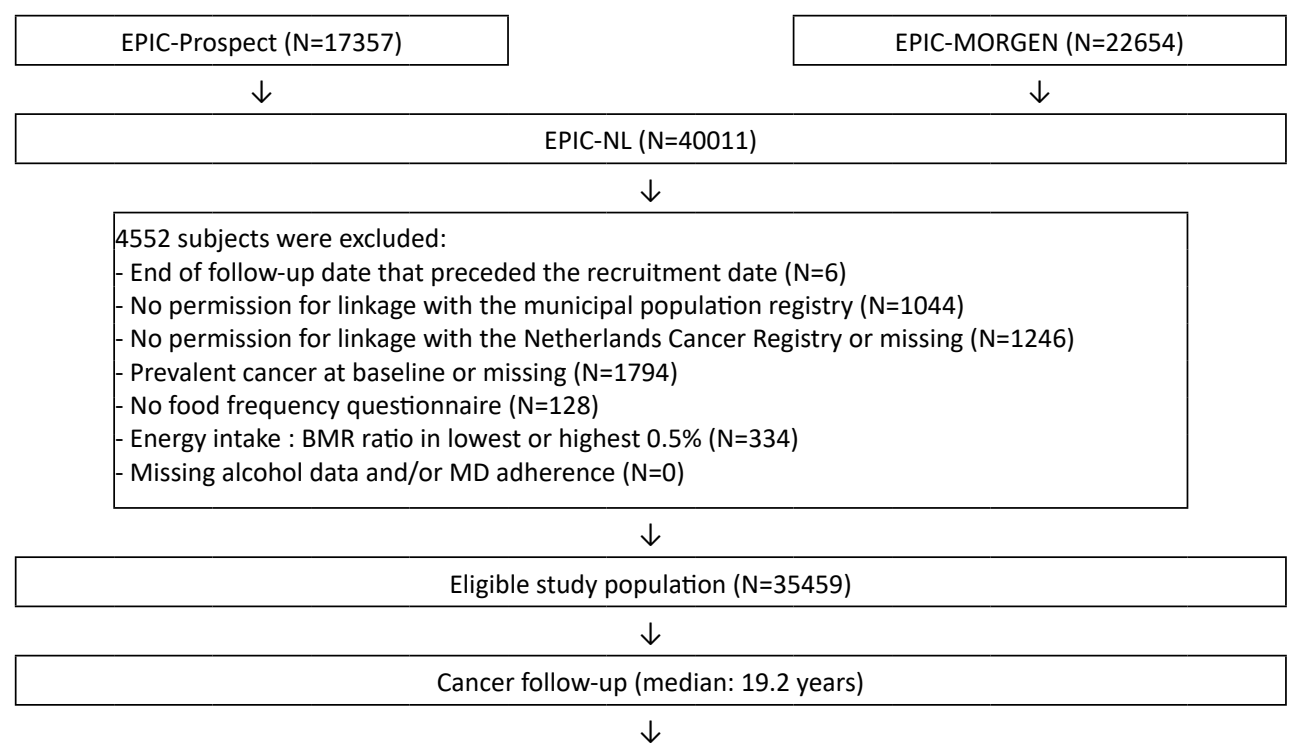

Exocrine pancreatic cancer cases

$(\mathrm{N}=142)$

\begin{tabular}{|cc|}
\hline & Microscopic confirmation status \\
\hline$\downarrow$ & $\downarrow$ \\
All pancreatic & Microscopically confirmed \\
cancer cases & pancreatic cancer cases \\
$(\mathrm{N}=142)$ & $(\mathrm{N}=104)$
\end{tabular}

Supplementary Figure S5.2 Flow diagram of the number of EPIC-NL participants, who are eligible for inclusion in the analyses

Abbreviations: EPIC, European Prospective Investigation into Cancer and Nutrition; EPIC-NL, the Dutch EPIC cohort; BMR, basal metabolic rate; MD, Mediterranean diet 


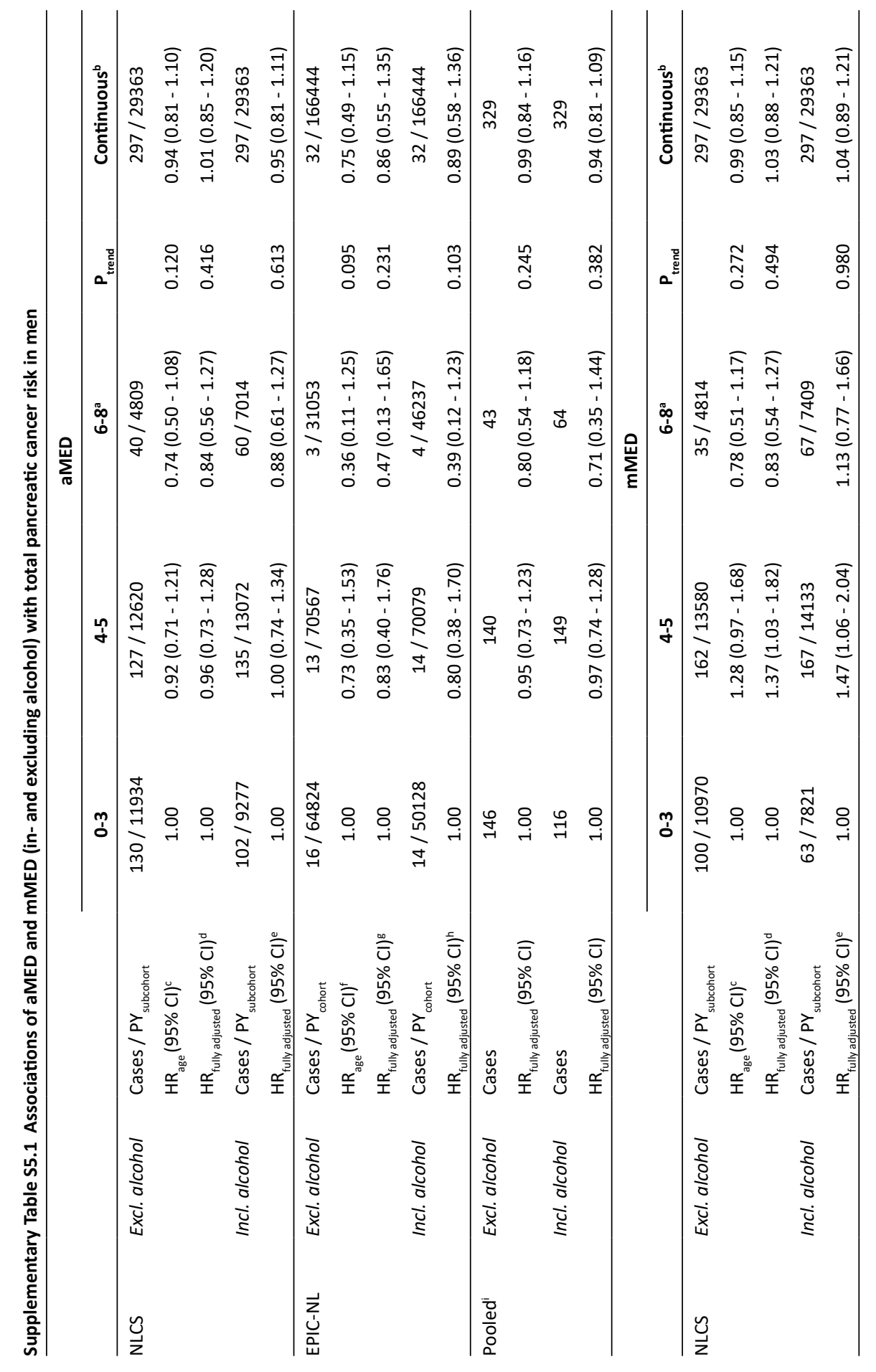




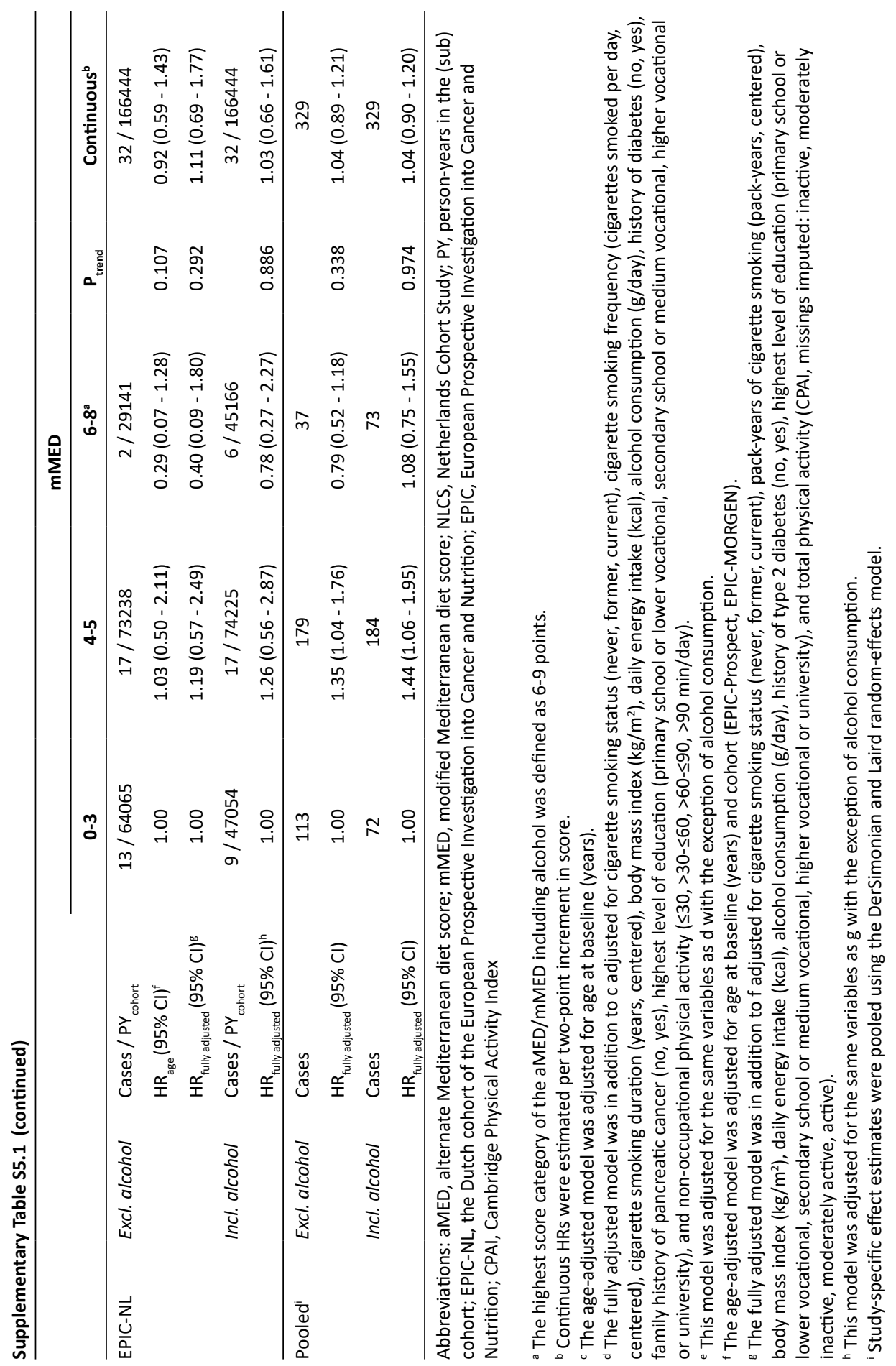




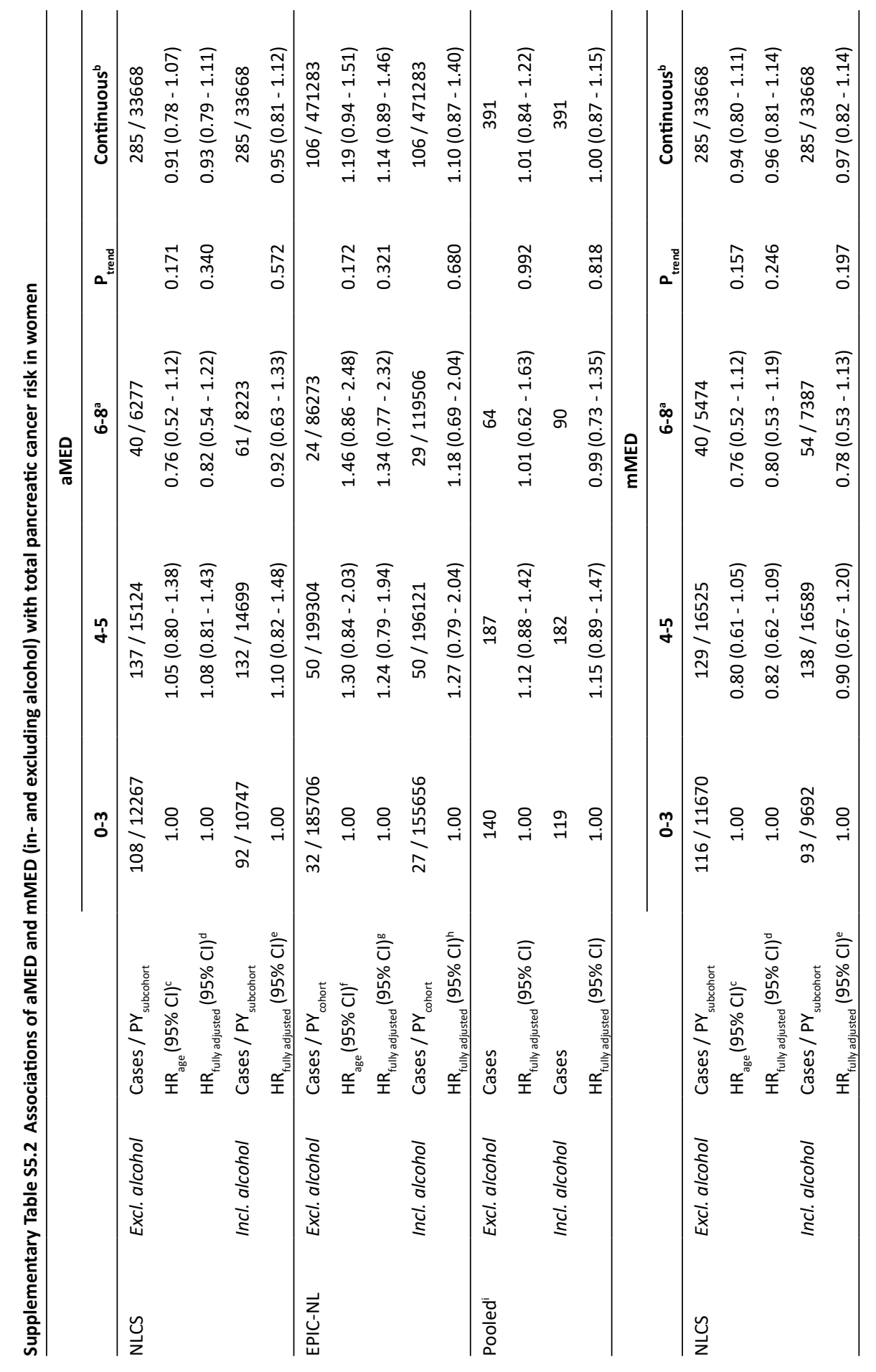




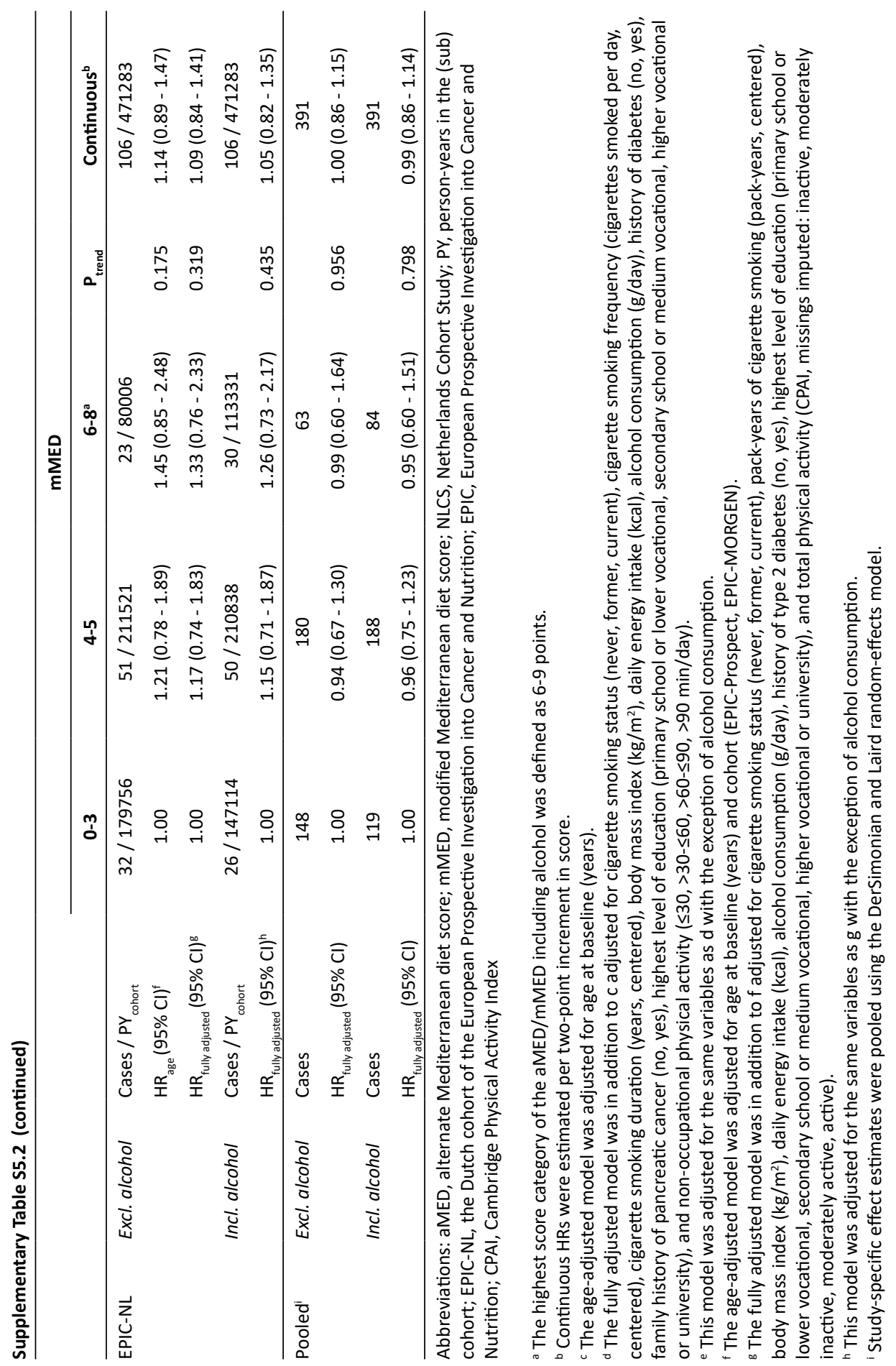




\section{Chapter 6}

\section{Mediterranean diet adherence and risk of colorectal cancer: the prospective Netherlands Cohort Study}

Maya Schulpen, Piet A. van den Brandt Eur J Epidemiol. 2020; 35(1): 25-35 


\begin{abstract}
Mediterranean diet (MD) adherence has been associated with a large variety of health benefits. However, prospective studies investigating the relation between MD adherence and colorectal cancer risk had inconsistent results. In this analysis of the Netherlands Cohort Study (NLCS), we evaluated sex- and subsite-specific associations of MD adherence with colorectal cancer risk. In 1986, 120852 subjects filled out the NLCS baseline questionnaire, which incorporated a 150-item food frequency questionnaire. MD adherence was estimated through alternate Mediterranean diet scores including and excluding alcohol (aMED and aMEDr, respectively). Using 20.3 year follow-up data, 1993 male and 1574 female colorectal cancer cases could be included in multivariable case-cohort analyses. aMEDr was not significantly associated with colorectal cancer risk, regardless of sex. Hazard ratios (95\% confidence intervals) per two-point increment were $1.04(0.95-1.13)$ for men and 0.97 (0.88 -1.07) for women. Additionally, there was no evidence of an inverse association with any of the colorectal cancer subsites (colon, proximal colon, distal colon, and rectum). In women, the association between aMEDr and colorectal cancer risk was significantly modified by smoking status $\left(p_{\text {interaction }}=0.015\right)$. Comparable results were obtained for the original aMED including alcohol. In conclusion, higher MD adherence was not associated with a reduced risk of colorectal cancer or anatomical subsites in the context of a Dutch population.
\end{abstract}




\section{Introduction}

Globally, colorectal cancer was an important contributor to the total cancer burden in 2018, ranking third and second in terms of incidence and mortality, respectively [1]. The global burden of colorectal cancer is expected to increase even further in the next decade. In 2030, over 2.2 million people are estimated to be diagnosed with colorectal cancer, whereas more than 1.1 million people are expected to die from this disease [2]. Colorectal cancer is a slow-growing disease [3], which offers the opportunity to intervene during the disease development process using preventive measures. These preventive strategies could for instance focus on maintenance of a healthy diet.

The traditional Mediterranean diet (MD), typical for the olive-cultivating areas bordering the Mediterranean basin in the early 1960s, has been associated with a large variety of health benefits, including decreases in all-cause mortality as well as cardiovascular disease risk and mortality [4-8]. This dietary pattern is characterized by the consumption of large quantities of vegetables, legumes, fruits, nuts, whole grains, and olive oil (rich in monounsaturated fatty acids, MUFA). In contrast, intakes of foods from animal origin (e.g., dairy and meat) are low. Finally, wine is consumed in moderate amounts, particularly during meals $[4,5]$.

The relation between a priori defined MD adherence and colorectal cancer risk has been evaluated in a number of prospective studies so far, with mixed results. Though some studies reported MD adherence to be associated with a significantly reduced colorectal cancer risk [9-12], inverse associations were absent or only observed in specific subgroups in others [13-20]. Additionally, heterogeneity of associations across the sexes and colorectal cancer subsites was indicated $[9,10,12,13,16,17,20]$.

The colorectum can anatomically be divided in the proximal colon, distal colon, and rectum. Depending on the anatomical subsite, colorectal tumors may develop through distinct molecular pathways and show varying patterns of (epi)genetic changes [21, 22]. Furthermore, differences have been shown in subsite-specific incidence trends and survival $[21,22]$. Because of their potentially distinct etiologies, cancers of the proximal colon, distal colon, and rectum should initially be considered as separate endpoints in epidemiological studies.

In the present analysis, we aimed to investigate associations of MD adherence with risks of colorectal cancer and anatomical subsites (colon, proximal colon, distal colon, and rectum) in the prospective Netherlands Cohort Study (NLCS). The level of MD adherence was assessed using a priori defined MD scores with and without alcohol component. Moreover, associations were estimated separately for men and women.

\section{Methods}

\section{Study population and cancer follow-up}

The NLCS was conducted among 58279 men and 62573 women, who were aged 55 to 69 years [23-26]. At baseline (September 1986), information on diet and other cancer risk factors was gathered via a self-administered questionnaire. Data were processed and analysed using the case-cohort method, in which cases are derived from the entire cohort and person-years at risk are estimated based on a subcohort. Therefore, a random subcohort $(\mathrm{N}=5000)$ was selected immediately after baseline and vital status information 
of subcohort members was acquired biennially [23, 26, 27]. Follow-up for cancer incidence was accomplished via annual record linkage with the Netherlands Cancer Registry and the nationwide Dutch Pathology Registry (PALGA) [24]. The NLCS was approved by institutional review boards from Maastricht University and the Netherlands Organization for Applied Scientific Research.

After 20.3 years of follow-up, 4084 subcohort members and 3966 cases with incident and microscopically confirmed colorectal cancer (ICD-O-3 codes: C18-C20) were eligible for inclusion in the present analyses (Figure 6.1). Eligible study participants did not have a history of cancer at baseline (except skin cancer), had complete and consistent dietary data, and had data available on alcohol consumption and MD adherence.

\section{Exposure assessment}

Habitual dietary intake during the year preceding baseline was assessed using a 150-item, semi-quantitative food frequency questionnaire (FFQ) $[25,28]$. Previously, it has been shown that this FFQ performed adequately and that dietary habits were reproducible for over at least five years [25, 28]. The 1986 Dutch food composition (NEVO) table was used to calculate nutrient intakes from the FFQ data [29].

\section{Mediterranean diet adherence}

MD adherence was assessed using the alternate Mediterranean diet score (aMED), which is a variant of the traditional Mediterranean diet score (tMED) developed by Trichopoulou et al., that was adapted for usage in the United States [30-33]. aMED assesses relative MD adherence based on energy-adjusted mean daily intakes of nine food groups with typically high or low consumption in the MD [32,33]. Each food group is scored by 0 or 1 points, creating a sum score with a maximum value of 9 points (highest level of MD adherence). Subjects receive 1 point for: high intakes ( $\geq$ sex-specific median) of vegetables (excluding potatoes), legumes, fruits, nuts, whole grains, and fish; a high ( $\geq$ sex-specific median) MUFA to saturated fatty acids (SFA) ratio; a moderate alcohol intake $(5-25 \mathrm{~g} /$ day); and a low intake (< sex-specific median) of red and processed meats [32, 33]. Moderate and heavy alcohol consumption have been associated with an increased colorectal cancer risk [34, 35]. Therefore, MD adherence was also assessed using a reduced variant of aMED (aMEDr) that does not include alcohol and ranges from 0 to 8 points. Because of the positive association between alcohol consumption and colorectal cancer risk, we will concentrate on results obtained using aMEDr in this article. MD score values were grouped into three MD adherence categories [low (0-3), middle (4-5), and high (6-8(9))] and were continuously modelled per two-point increment [33].

\section{Statistical analyses}

Cox proportional hazards analyses were conducted to estimate hazard ratios (HRs) and 95\% confidence intervals $(95 \% \mathrm{Cls})$ for sex-specific associations of MD adherence with incidences of total colorectal cancer and anatomical subsites (colon, proximal colon, distal colon, and rectum). Duration of follow-up was used as time variable and person-years at risk of subcohort members were calculated from baseline until colorectal cancer diagnosis, death, emigration, loss to follow-up, or end of follow-up, whichever came first. To account for the 


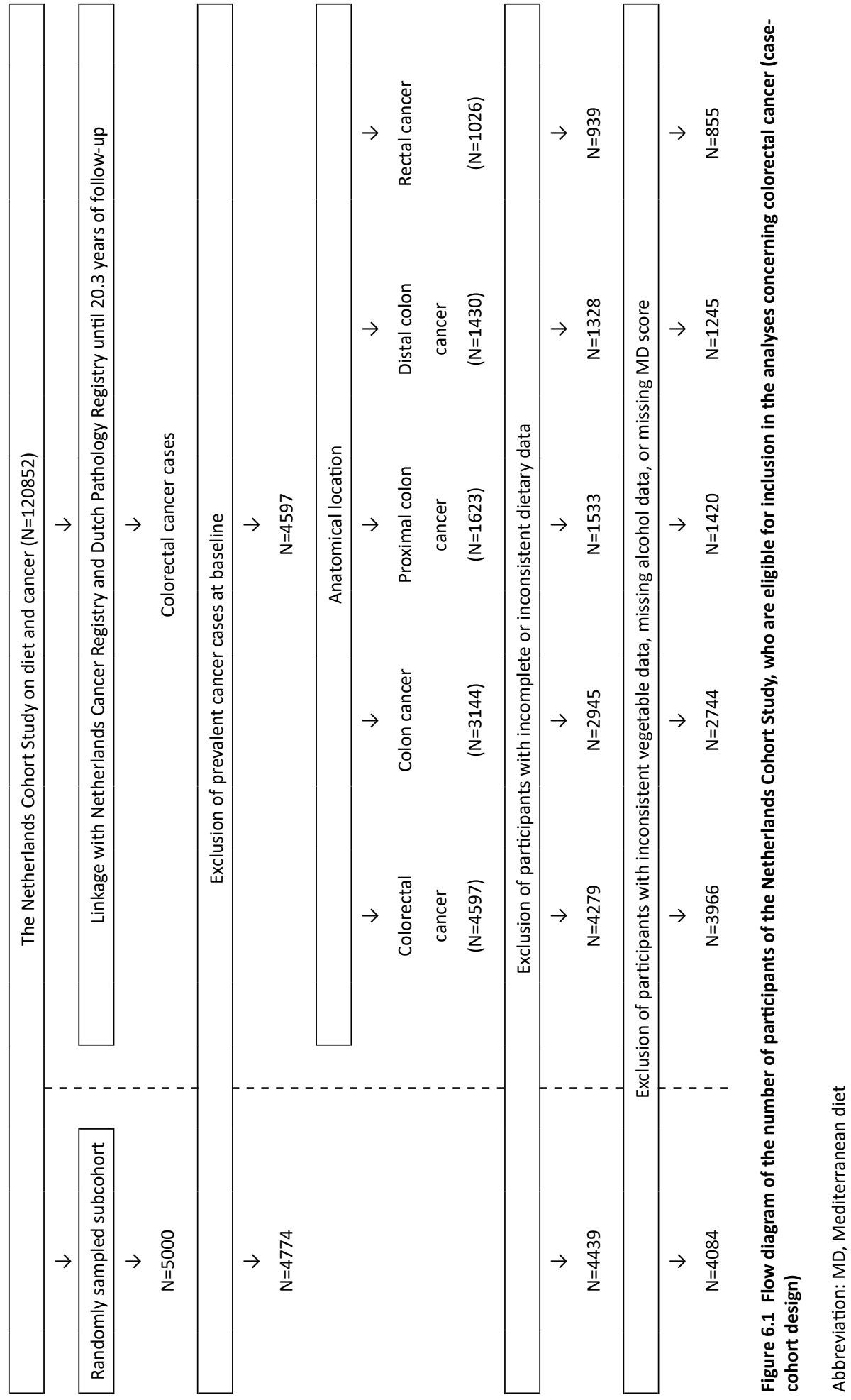


increased variance inherent to the case-cohort design, we estimated standard errors using the robust Huber-White sandwich estimator [36]. Scaled Schoenfeld residuals tests and $\ln (-\ln )$ survival plots confirmed that it was appropriate to assume proportionality of hazards for the exposure variables [37].

MD scores were included as categorical and continuous terms in age- and multivariableadjusted Cox models. Based on the literature, we included the following predefined confounders in the multivariable-adjusted models: age at baseline, cigarette smoking behaviour (status, frequency, and duration), body mass index (BMI), alcohol consumption (except for models containing the original aMED including alcohol), total daily energy intake, highest level of education, non-occupational physical activity, and family history of colorectal cancer. Other covariates considered were height, history of diabetes, history of chronic bowel irritation, use of hormone replacement therapy (women only), and long-term use of non-steroidal anti-inflammatory drugs. These factors did not change the HR estimates of aMEDr $\geq 10 \%$ and were therefore not included in the final model. P-values for trends over the MD adherence categories were obtained by appointing sexspecific median MD score values among subcohort members to each category and fitting these as continuous terms in Cox regression models. Performances of models including MD score variants with and without alcohol (aMED and aMEDr, respectively) were compared using Akaike's Information Criterion (AIC) [38]. Statistical significance of differences in associations with aMEDr across the anatomical locations of colorectal cancer (colon, proximal colon, distal colon, and rectum) was tested using a competing risks procedure, by which standard errors were estimated using a bootstrapping method developed for the case-cohort design $[39,40]$.

Stratified analyses were performed to evaluate associations of aMEDr with colorectal cancer risk across levels of cigarette smoking status, alcohol consumption, BMI, educational level, and family history of colorectal cancer. Interaction terms between aMEDr and these potential effect modifiers were added to the models to test the statistical significance of potential differences. To test the sensitivity of our results, analyses were repeated excluding the first two years of follow-up. Furthermore, the total follow-up time was divided into three periods $(\leq 2,>2-\leq 10$, and $>10$ years).

As an additional sensitivity analysis, we compared the population-dependent aMED to the absolute WCRF/AICR diet score, which is based on the dietary recommendations for cancer prevention issued by the World Cancer Research Fund/American Institute for Cancer Research (WCRF/AICR) in 2007 [41]. Our WCRF/AICR diet score is based on the WCRF/AICR score developed in the European Prospective Investigation into Cancer and Nutrition (EPIC) cohort $[42,43]$ and operationalizes the recommendations concerning foods and drinks that promote weight gain, plant foods, red and processed meats, (alcohol), and salt. A detailed description of the calculation of the score has been published previously [44]. Score variants were created including and excluding the alcohol recommendation, resulting in sum scores ranging from 0 to 4 (or 5 when including alcohol) points with higher values reflecting closer adherence to the WCRF/AICR dietary recommendations. Cox regression analyses were performed to estimate multivariable-adjusted associations of the WCRF/AICR diet scores (per SD-increment) with risks of colorectal, colon, and rectal cancer. A similar approach was applied to the aMED indices to be able to compare model performances of both scores using AIC. Statistical analyses were conducted using Stata (version 15). Statistically significant results had a two-sided $\mathrm{p}$-value below 0.05 . 


\section{Results}

Sex-specific median daily intakes of the aMEDr components among subcohort members are displayed in Table 6.1. As expected, median daily intakes of beneficial components increased with higher levels of MD adherence, whereas the opposite was observed for the intake of red and processed meats. Alcohol consumption was constant over the aMEDr categories in men, whereas in women slightly higher intakes were observed with closer adherence to the MD. Distributions of potential (colorectal) cancer risk factors (e.g., smoking status, BMI, and physical activity) over the aMEDr categories in the NLCS subcohort have been described in detail previously [45]. Comparing the highest to the lowest aMEDr category, subcohort members adhering more closely to the MD were less likely to smoke at baseline, had a lower $\mathrm{BMI}$, and were more physically active. Generally, comparable levels of MD adherence were observed among colorectal cancer cases and subcohort members of both sexes, with mean aMEDr values of approximately 4 (Table 6.2). Considering other baseline characteristics, male and female colorectal cancer cases were more often former smokers compared to subcohort members, but less often current smokers (except female rectal cancer cases, Table 6.2). Additionally, levels of physical activity and alcohol consumption were higher in male, but lower in female, colorectal cancer cases. Furthermore, colon cancer cases were more likely to be highly educated than subcohort members (men only), whereas the opposite was observed for rectal cancer cases. Finally, colorectal cancer cases of both sexes more frequently reported a family history of this disease.

Table 6.3 presents sex-specific and multivariable-adjusted associations of aMED, including and excluding alcohol, with risks of colorectal cancer and anatomical subsites. Age-adjusted associations can be found in Supplementary Table S6.1. Not all eligible subjects could be included in the Cox models because of missing information on covariates.

In men, aMEDr was not significantly associated with colorectal cancer risk in categorical and continuous analyses $\left[\mathrm{HR}_{\text {per two-point increment }}(95 \% \mathrm{Cl}): 1.04\right.$ (0.95-1.13)] (Table 6.3). Subsitespecific HR estimates per two-point increment in aMEDr (all not statistically significant) ranged from 0.98 for distal colon cancer to 1.11 for rectal cancer and did not significantly differ $\left[p_{\text {heterogeneity }}: 0.566\right.$ (proximal vs. distal colon) and 0.518 (colon vs. rectum)]. Similar to men, no significant association was observed between aMEDr and colorectal cancer risk in women $\left[\mathrm{HR}_{\text {per two-point increment }}(95 \% \mathrm{Cl}): 0.97\right.$ (0.88 - 1.07)] (Table 6.3). However, middle vs. low aMEDr values were associated with a borderline significantly reduced colorectal cancer risk [HR (95\% Cl): $0.86(0.73-1.00)]$. Though subsite-specific associations were all not statistically significant and there was no evidence of heterogeneity $\left[\mathrm{p}_{\text {heterogeneity }}: 0.690\right.$ (proximal vs. distal colon) and 0.194 (colon vs. rectum)], results suggested a weak inverse association between aMEDr and rectal cancer risk in women $\left[\mathrm{HR}_{\text {per two-point increment }}(95 \% \mathrm{Cl}): 0.91(0.76-1.08)\right]$. Comparable results were obtained for the original aMED including alcohol in both men and women (Table 6.3). For colorectal cancer risk, inclusion of alcohol in the MD score resulted in a worse model fit.

Associations of aMEDr with colorectal cancer risk in women differed statistically significantly across strata of smoking status ( $p_{\text {interaction }}=0.015$, Table 6.4). In female ex-smokers, increasing aMEDr values were associated with a significantly reduced colorectal cancer risk $\left[\mathrm{HR}_{\text {per two- }}\right.$ $(95 \% \mathrm{Cl}): 0.78(0.63-0.98)]$. In contrast, a positive association was suggested in female current smokers, with a significant positive trend over the aMEDr categories $\left(p_{\text {trend }}=0.04\right.$, data not shown). Finally, there was no evidence of an association in women who had never smoked. No significant interactions were observed between aMEDr and other 


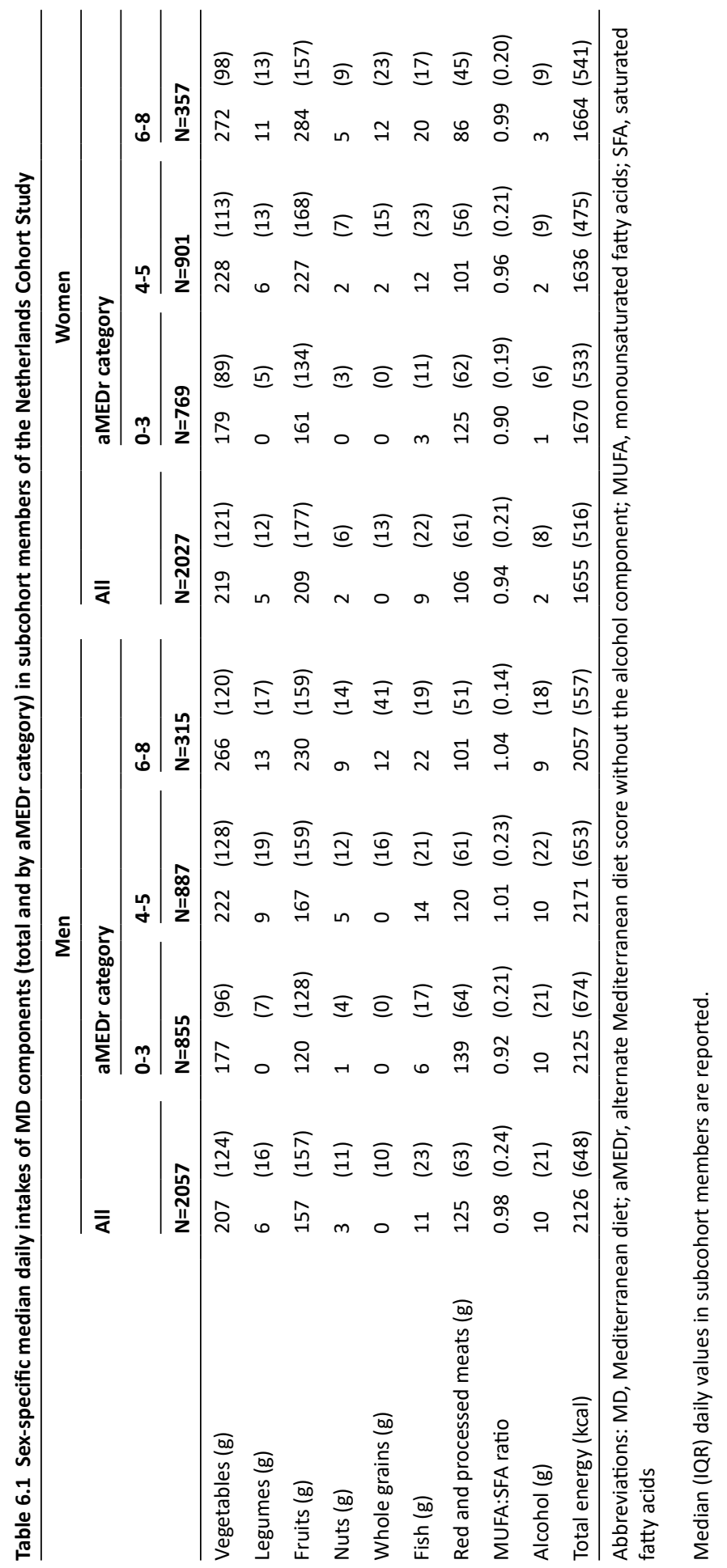




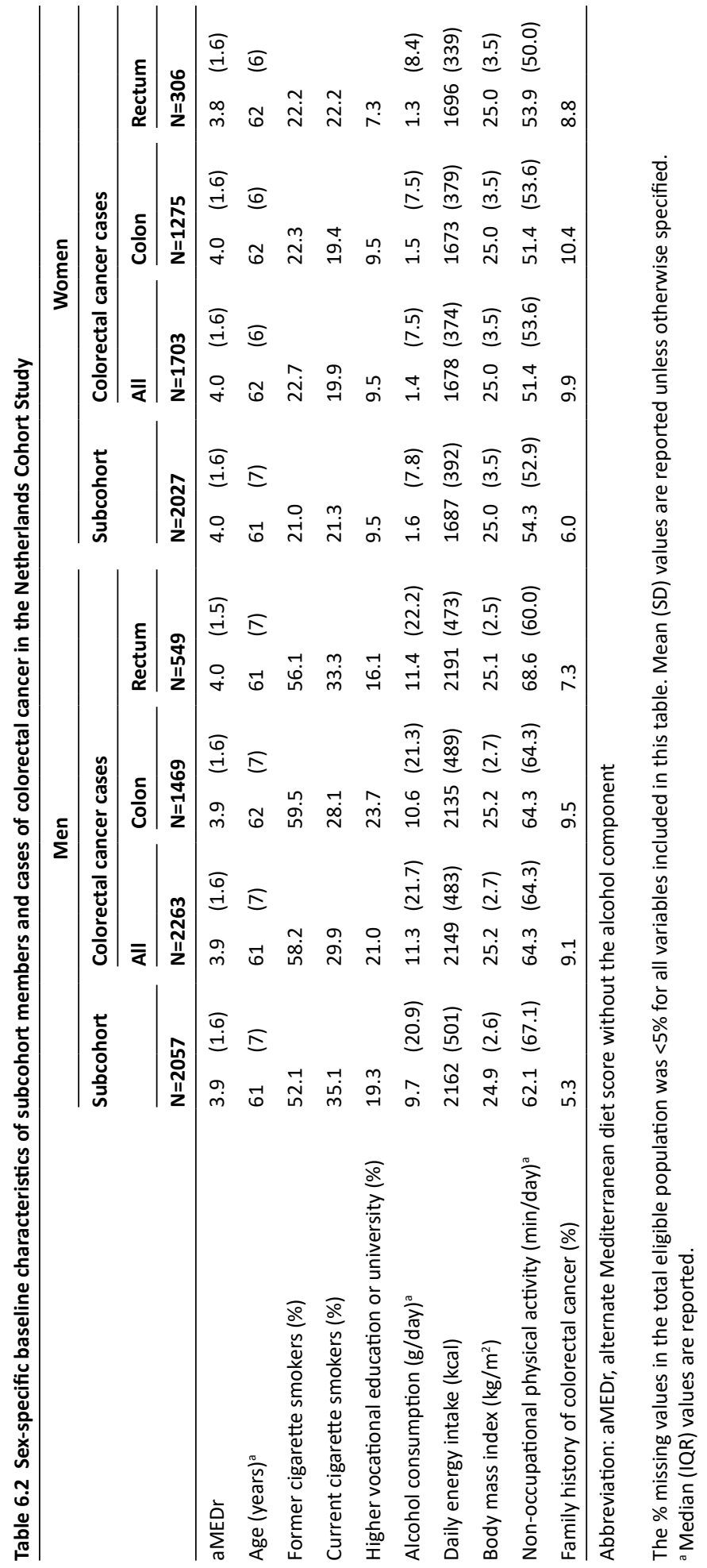




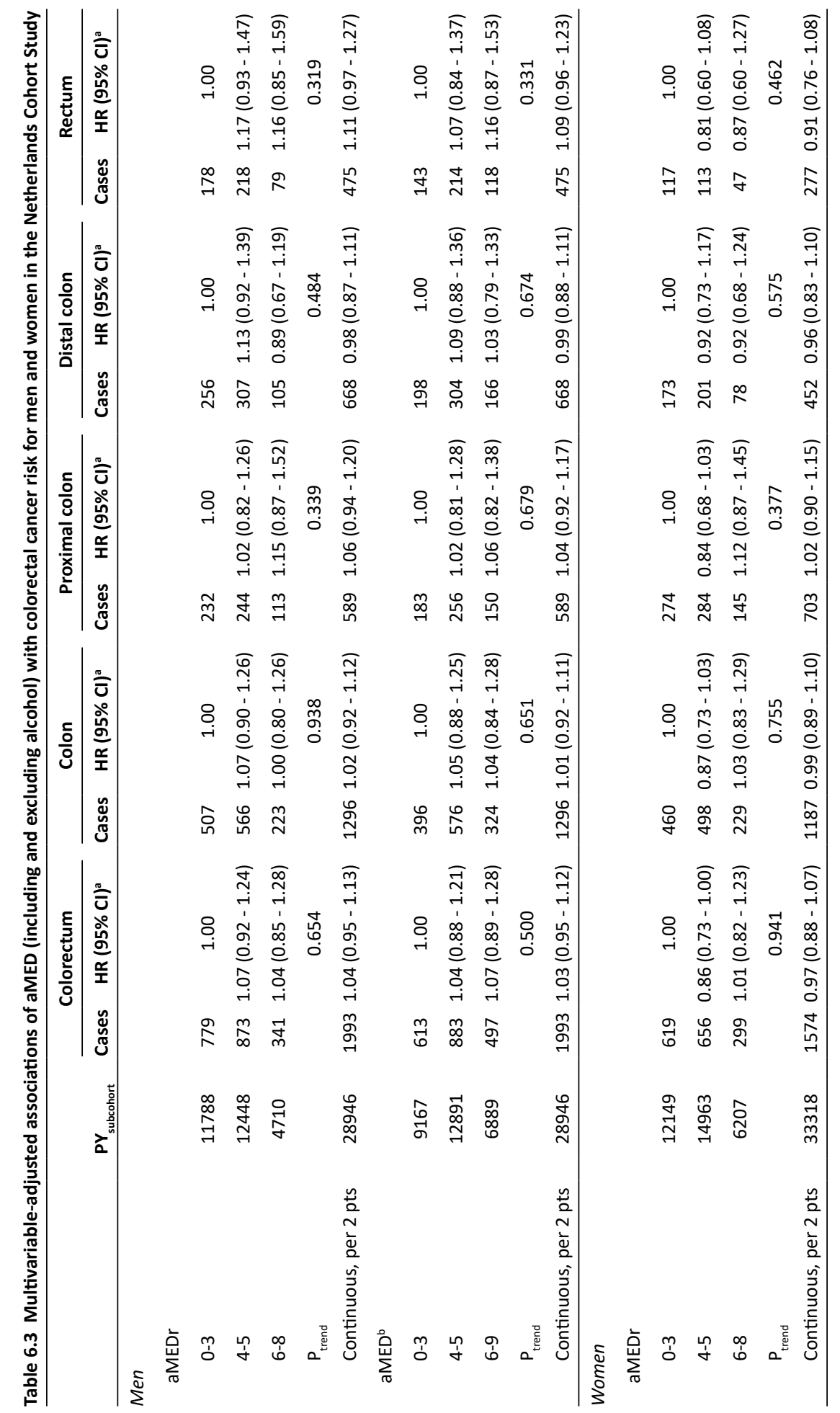




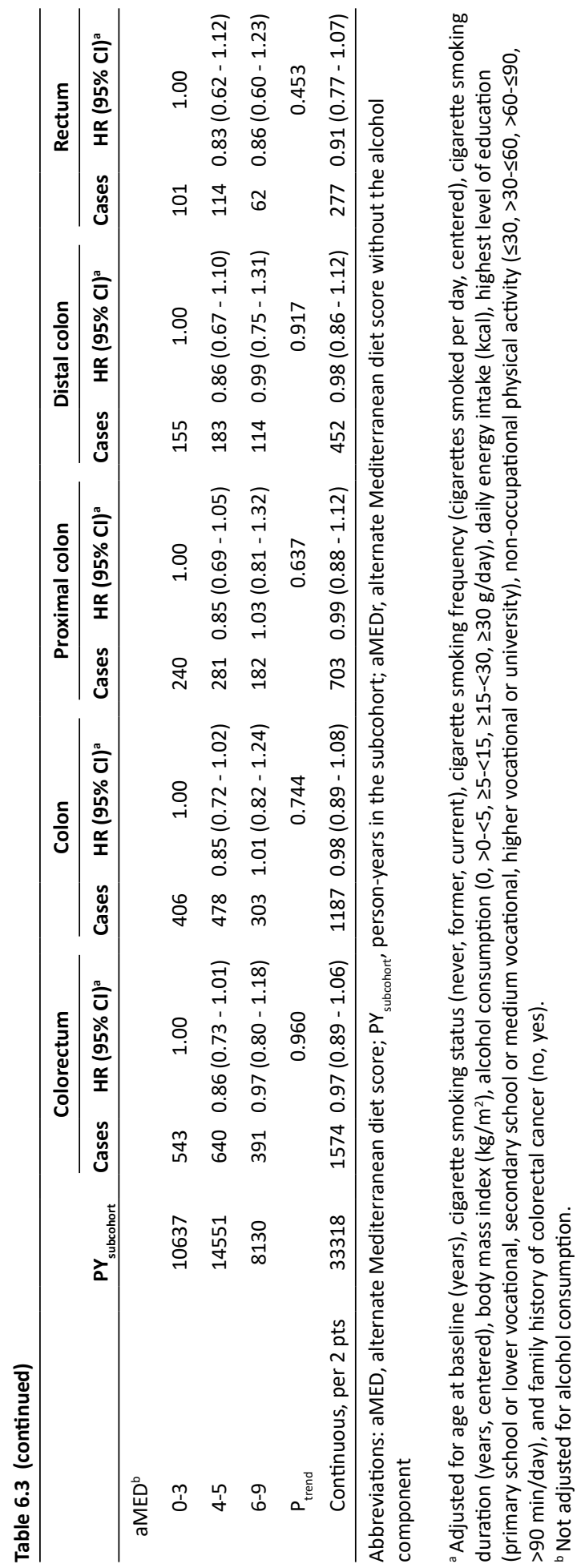


potential colorectal cancer risk factors (alcohol consumption, BMI, educational level, and family history of colorectal cancer) in men and women, or smoking status in men (Table 6.4). Simultaneous inclusion of all aMEDr components as dichotomous variables in multivariableadjusted models showed that none of the individual components was significantly associated with colorectal cancer risk (data not shown). Associations were comparable after exclusion of the first two years of follow-up and did not significantly differ across the three follow-up periods (data not shown).

Like the population-dependent aMED indices, the absolute WCRF/AICR diet scores (including and excluding alcohol) were not significantly associated with risks of colorectal, colon, and rectal cancer in men and women (Table 6.5). Performances of models containing aMED indices and WCRF/AICR diet scores were mostly comparable.

\section{Discussion}

In this prospective cohort study, a priori defined MD adherence, assessed by aMEDr, was not significantly associated with colorectal cancer risk. Associations were absent for all investigated anatomical subsites and in both men and women. The association between aMEDr and colorectal cancer risk in women was significantly modified by smoking status $\left(p_{\text {interaction }}=0.015\right)$. A significant inverse association was observed in female ex-smokers, whereas a positive association was suggested in female current smokers. For colorectal cancer risk, the best model performance was obtained when alcohol intake was not included in the MD score.

Various prospective cohorts have investigated the relation of a priori defined MD adherence with colorectal cancer risk and indicated disparate associations for men and women. In men, higher MD adherence has fairly consistently been associated with a reduced colorectal cancer risk (but not always significant) $[9,10,13,16,17,20]$. For example, in male participants of the Multiethnic Cohort Study (MEC), the National Institutes of Health-American Association of Retired Persons (NIH-AARP) Diet and Health Study, and the Health Professionals Followup Study (HPFS), statistically significant HR estimates of $0.84,0.72$, and 0.80 , respectively, were obtained when comparing high to low aMED values [13, 16, 20]. Furthermore, high compared to low MD adherence (modified Mediterranean diet score) was associated with a non-significantly reduced colorectal cancer risk in the male part of the EPIC cohort [HR $195 \%$ Cl): $0.89(0.76-1.04)$ ] [10]. With some exceptions [9, 12], studies in women did not support the presence of an inverse association between MD adherence and colorectal cancer risk $[10,13,15-18,20]$. For comparison, non-significant HR estimates of 0.96 (MEC), 0.89 (NIHAARP), 0.99 (Nurses' Health Study, NHS), and 0.88 (EPIC), were reported for high vs. low MD adherence in female participants of the abovementioned studies $[10,13,16,20]$. In the present analysis of the NLCS, a priori defined MD adherence was not associated with a significantly decreased risk of colorectal cancer in both sexes. Similar to our analysis, the majority of the previously conducted studies used aMED (variants) to assess MD adherence. However, the particular food items included in the aMED components may have differed between studies, which could (partly) explain the contrasting results that we observed for men in our cohort. Additionally, the more homogenous nature of the NLCS study population may have resulted in relatively small contrasts in absolute food intakes between subjects in the highest and lowest adherence categories making it more difficult to detect potentially beneficial effects of the MD on health outcomes. Median daily intakes among male NLCS subcohort members in the highest and lowest aMEDr categories were for example $266 \mathrm{~g}$ and 
Table 6.4 Sex-specific and multivariable-adjusted associations of aMEDr (per two-point increment) with colorectal cancer risk for various subgroups in the Netherlands Cohort Study

\begin{tabular}{|c|c|c|c|c|}
\hline & \multicolumn{4}{|c|}{ Colorectal cancer } \\
\hline & \multicolumn{2}{|r|}{ Men } & \multicolumn{2}{|r|}{ Women } \\
\hline & Cases & $\mathrm{HR}(95 \% \mathrm{Cl})^{\mathrm{a} b}$ & Cases & HR $(95 \% \mathrm{Cl})^{\mathrm{a} b}$ \\
\hline \multicolumn{5}{|l|}{ Cigarette smoking status ${ }^{c}$} \\
\hline Never & 256 & $1.11(0.87-1.41)$ & 915 & $1.00(0.88-1.13)$ \\
\hline Former & 1184 & $1.03(0.92-1.15)$ & 350 & $0.78(0.63-0.98)$ \\
\hline Current & 553 & $1.02(0.85-1.22)$ & 309 & $1.21(0.96-1.51)$ \\
\hline$P_{\text {interaction }}{ }^{d}$ & & 0.714 & & 0.015 \\
\hline \multicolumn{5}{|l|}{ Alcohol consumption ${ }^{e}$} \\
\hline $0 \mathrm{~g} / \mathrm{day}$ & 235 & $1.14(0.90-1.46)$ & 489 & $1.04(0.86-1.25)$ \\
\hline$>0-<15.0 \mathrm{~g} /$ day & 934 & $1.05(0.92-1.19)$ & 865 & $0.97(0.86-1.10)$ \\
\hline$\geq 15.0 \mathrm{~g} /$ day & 824 & $0.98(0.85-1.13)$ & 220 & $0.88(0.66-1.17)$ \\
\hline$P_{\text {interaction }}{ }^{d}$ & & 0.731 & & 0.539 \\
\hline \multicolumn{5}{|l|}{ Body mass index ${ }^{f}$} \\
\hline$\geq 18.5-<25.0 \mathrm{~kg} / \mathrm{m}^{2}$ & 970 & $1.04(0.92-1.17)$ & 848 & $1.03(0.90-1.17)$ \\
\hline$\geq 25.0 \mathrm{~kg} / \mathrm{m}^{2}$ & 1018 & $1.05(0.92-1.19)$ & 707 & $0.90(0.78-1.04)$ \\
\hline$P_{\text {interaction }}{ }^{d}$ & & 0.876 & & 0.232 \\
\hline \multicolumn{5}{|l|}{ Highest level of education ${ }^{g}$} \\
\hline Primary school or lower vocational & 863 & $0.97(0.84-1.12)$ & 841 & $0.95(0.84-1.08)$ \\
\hline Secondary school or medium vocational & 706 & $1.04(0.90-1.19)$ & 579 & $1.00(0.84-1.19)$ \\
\hline Higher vocational or university & 424 & $1.21(0.98-1.50)$ & 154 & $1.08(0.79-1.47)$ \\
\hline$P_{\text {interaction }}{ }^{d}$ & & 0.133 & & 0.920 \\
\hline \multicolumn{5}{|l|}{ Family history of colorectal cancer ${ }^{\mathrm{h}}$} \\
\hline No & 1811 & $1.06(0.97-1.16)$ & 1412 & $0.99(0.89-1.09)$ \\
\hline Yes & 182 & $0.82(0.55-1.23)$ & 162 & $0.90(0.59-1.39)$ \\
\hline$P_{\text {interaction }}{ }^{d}$ & & 0.204 & & 0.423 \\
\hline
\end{tabular}

Abbreviation: aMEDr, alternate Mediterranean diet score without the alcohol component

${ }^{\text {a }}$ All HRs were estimated per two-point increment in aMEDr.

${ }^{\mathrm{b}}$ Adjusted for age at baseline (years), cigarette smoking status (never, former, current), cigarette smoking frequency (cigarettes smoked per day, centered), cigarette smoking duration (years, centered), body mass index $\left(\mathrm{kg} / \mathrm{m}^{2}\right)$, alcohol consumption $(0,>0-<5, \geq 5-<15, \geq 15-<30, \geq 30 \mathrm{~g} /$ day), daily energy intake (kcal), highest level of education (primary school or lower vocational, secondary school or medium vocational, higher vocational or university), non-occupational physical activity $(\leq 30,>30-\leq 60,>60-\leq 90,>90 \mathrm{~min} /$ day $)$, and family history of colorectal cancer (no, yes).

${ }^{\mathrm{C}}$ Not adjusted for cigarette smoking status.

dP-values for interaction were obtained by testing the statistical significance of interaction terms between aMEDr and the stratifying covariates in multivariable-adjusted models.

e Not adjusted for alcohol consumption.

${ }^{\mathrm{f}}$ Not adjusted for body mass index.

${ }^{\mathrm{g}}$ Not adjusted for highest level of education.

${ }^{\mathrm{h}}$ Not adjusted for family history of colorectal cancer. 


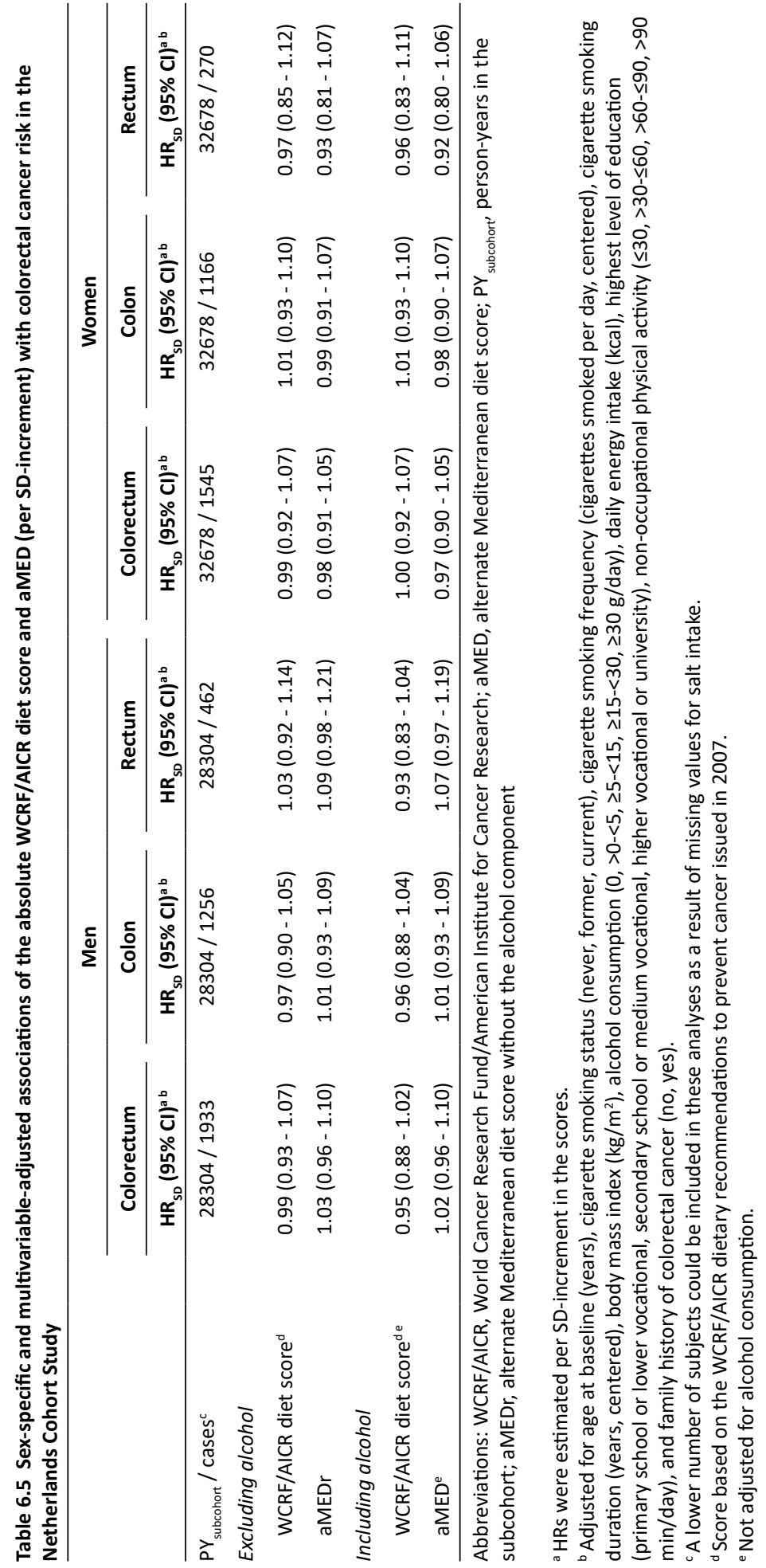


$177 \mathrm{~g}$ for vegetables, $230 \mathrm{~g}$ and $120 \mathrm{~g}$ for fruits, and $101 \mathrm{~g}$ and $139 \mathrm{~g}$ for red and processed meats, respectively. In male participants of the HPFS [20], the mean numbers of servings per day in the highest and lowest aMED quintiles were 4.9 and 2.0 for vegetables, 2.6 and 0.8 for fruits, and 0.7 and 1.2 for red and processed meats. We calculated ratios comparing median/mean intakes in the highest and lowest MD categories. The ratios showed clearly higher contrasts in intakes of vegetables and fruits in the HPFS [vegetables: 1.5 (NLCS) vs. 2.5 (HPFS), fruits: 1.9 (NLCS) vs. 3.3 (HPFS)]. The contrast in the intake of red and processed meats was comparable in both cohorts [0.7 (NLCS) vs. 0.6 (HPFS)]. We were forced to compare median daily intakes in the NLCS with mean numbers of servings per day in the HPFS, because there were no other data available. Despite our relatively homogeneous study population, we previously detected significant inverse associations between aMEDr and risks of esophageal squamous cell carcinoma, gastric cardia adenocarcinoma (GCA), and gastric non-cardia adenocarcinoma (GNCA) in men in the NLCS [46], suggesting sufficient contrast. In female NLCS participants, associations of aMEDr with risks of GCA and GNCA were also inverse, but did not reach statistical significance.

None of the aMEDr components was individually associated with colorectal cancer risk in the present study. Possibly, the individual effects of the aMEDr components were too weak to be detected. Combining these components into a dietary pattern score increases the likelihood that the potentially weak individual effects are being detected. Furthermore, by investigating the effect of a dietary pattern, one allows for synergistic or antagonistic interactions between the dietary components, and solves confounding and collinearity problems associated with the analysis of single food groups. Finally, the contrast within the study population in terms of overall healthiness of the diet is possibly increased when considering the MD as a whole, which increases the chance of detecting true effects, if present $[31,47,48]$.

The potentially distinct etiological backgrounds of tumors arising in the proximal colon, distal colon, and rectum and varying exposures to (carcinogens in) fecal matter across subsites may cause heterogeneous susceptibilities to (lifestyle) risk factors [21, 49]. Subsite-specific analyses in the NIH-AARP and HPFS cohorts demonstrated that the inverse association between $\mathrm{MD}$ adherence and colorectal cancer risk in men was particularly pronounced for distal colon cancer and rectal cancer $[13,20]$. However, associations did not seem to differ across the subsites in men in our study. Additionally, there was no clear evidence for heterogeneity across the anatomical subsites in women, both in our cohort and in most previous studies $[13,15,20]$.

In women in our cohort, associations between MD adherence and colorectal cancer risk significantly differed across strata of smoking status, with oppositely directed associations being observed in former smokers (inverse) and current smokers (positive). Smoking status did not significantly interact with MD adherence in female participants of the NHS [20]. However, this study did not differentiate between former and current smokers. A possible explanation for the interaction with smoking status that we observed is chance, considering the large number of tests performed. We recommend that the potentially modifying role of smoking status in the association between MD adherence and colorectal cancer risk, as well as underlying mechanisms, are investigated in future studies. Preferably, these studies should be performed separately for men and women, and distinguish between former and current smokers.

Colorectal cancers usually develop slowly over the course of 10 to 15 years [3], making 
the prospective design and long duration of follow-up major strengths of the present study. The large number of cases diagnosed during follow-up facilitated the performance of sex-specific analyses for cancers of the colorectum, colon, proximal colon, distal colon, and rectum with acceptable statistical power, while adjusting for relevant confounders. Additionally, associations were estimated within strata of colorectal cancer risk factors, separately for men and women. Since the national population screening program for colorectal cancer in the Netherlands started after the end of follow-up of our study [50], it could not have influenced the results. Despite the high quality of the dietary information, possible measurement error may have attenuated associations. Another limitation is the single measurement of diet and lifestyle factors at baseline. Changes in diet and lifestyle factors during follow-up may have led to non-differential misclassification and attenuated associations. However, the baseline assessment of the NLCS-FFQ has been shown to be capable of ranking subjects according to their nutrient intakes relatively well for over at least five years [28]. Furthermore, associations between aMEDr and colorectal cancer risk were largely similar, and did not significantly differ, across the three periods of follow-up $(\leq 2,>2$ $\leq 10$, and $>10$ years). Residual confounding by unmeasured factors also cannot be excluded. Lastly, aMEDr assesses the relative level of MD adherence using population-based cut-offs. Therefore, subjects with high scores do not necessarily adhere closely to a traditional MD, particularly in non-Mediterranean study populations. Comparison of diets of the Netherlands and Greece using previously reported intake data from the EPIC cohort [51] showed that mean daily intakes of food groups typically consumed in large amounts in the MD, such as vegetables, fruits, and legumes, were lower in participants of the Dutch EPIC cohorts (EPIC$\mathrm{NL}$ ) compared to participants of the Greek EPIC cohort (EPIC-Greece). Mean daily intakes of vegetables, fruits, and legumes among men were $131 \mathrm{~g}, 156 \mathrm{~g}$, and $6 \mathrm{~g}$ in EPIC-NL and $269 \mathrm{~g}, 234 \mathrm{~g}$, and $33 \mathrm{~g}$ in EPIC-Greece, respectively. Among female participants of EPIC-NL and EPIC-Greece, mean daily intakes were $128 \mathrm{~g}$ and $211 \mathrm{~g}$ for vegetables, $183 \mathrm{~g}$ and $218 \mathrm{~g}$ for fruits, and $4 \mathrm{~g}$ and $21 \mathrm{~g}$ for legumes, respectively. As expected, meat consumption was higher in Dutch subjects [EPIC-NL: $141 \mathrm{~g}$ (men) and $80 \mathrm{~g}$ (women), EPIC-Greece: $68 \mathrm{~g}$ (men) and $35 \mathrm{~g}$ (women)] [51]. Regardless of its use of population-based cut-offs, the model fit of aMEDr was generally comparable to that of the absolute WCRF/AICR diet score in our study.

In conclusion, results of this large prospective cohort study do not support the hypothesis that higher MD adherence is associated with a reduced risk of colorectal cancer. MD adherence was not significantly associated with the risk of any of the colorectal cancer subsites in both men and women. 


\section{References}

1. Bray F, Ferlay J, Soerjomataram I, Siegel RL, Torre LA, Jemal A. Global cancer statistics 2018: GLOBOCAN estimates of incidence and mortality worldwide for 36 cancers in 185 countries. CA Cancer J Clin. 2018;68(6):394-424.

2. Arnold M, Sierra MS, Laversanne M, Soerjomataram I, Jemal A, Bray F. Global patterns and trends in colorectal cancer incidence and mortality. Gut. 2017;66(4):683-91.

3. Kuipers EJ, Grady WM, Lieberman D, Seufferlein T, Sung JJ, Boelens PG, et al. Colorectal cancer. Nat Rev Dis Primers. 2015;1:15065.

4. Willett WC, Sacks F, Trichopoulou A, Drescher G, Ferro-Luzzi A, Helsing E, et al. Mediterranean diet pyramid: a cultural model for healthy eating. Am J Clin Nutr. 1995;61(6 Suppl):1402S-6S.

5. Trichopoulou A, Lagiou P. Healthy traditional Mediterranean diet: an expression of culture, history, and lifestyle. Nutr Rev. 1997;55(11 Pt 1):383-9.

6. Martinez-Gonzalez MA, Bes-Rastrollo M. Dietary patterns, Mediterranean diet, and cardiovascular disease. Curr Opin Lipidol. 2014;25(1):20-6.

7. Sofi F, Macchi C, Abbate R, Gensini GF, Casini A. Mediterranean diet and health status: an updated meta-analysis and a proposal for a literature-based adherence score. Public Health Nutr. 2014;17(12):2769-82.

8. Grosso G, Marventano S, Yang J, Micek A, Pajak A, Scalfi L, et al. A comprehensive metaanalysis on evidence of Mediterranean diet and cardiovascular disease: Are individual components equal? Crit Rev Food Sci Nutr. 2017;57(15):3218-32.

9. Agnoli C, Grioni S, Sieri S, Palli D, Masala G, Sacerdote C, et al. Italian Mediterranean Index and risk of colorectal cancer in the Italian section of the EPIC cohort. Int J Cancer. 2013;132(6):140411.

10. Bamia C, Lagiou P, Buckland G, Grioni S, Agnoli C, Taylor AJ, et al. Mediterranean diet and colorectal cancer risk: results from a European cohort. Eur J Epidemiol. 2013;28(4):317-28.

11. Fasanelli F, Zugna D, Giraudo MT, Krogh V, Grioni S, Panico S, et al. Abdominal adiposity is not a mediator of the protective effect of Mediterranean diet on colorectal cancer. Int J Cancer. 2017;140(10):2265-71.

12. Jones P, Cade JE, Evans CEL, Hancock N, Greenwood DC. The Mediterranean diet and risk of colorectal cancer in the UK Women's Cohort Study. Int J Epidemiol. 2017;46(6):1786-96.

13. Reedy J, Mitrou PN, Krebs-Smith SM, Wirfalt E, Flood A, Kipnis V, et al. Index-based dietary patterns and risk of colorectal cancer: the NIH-AARP Diet and Health Study. Am J Epidemiol. 2008;168(1):38-48.

14. Fung TT, Hu FB, Wu K, Chiuve SE, Fuchs CS, Giovannucci E. The Mediterranean and Dietary Approaches to Stop Hypertension (DASH) diets and colorectal cancer. Am J Clin Nutr. 2010;92(6):142935.

15. Vargas AJ, Neuhouser ML, George SM, Thomson CA, Ho GY, Rohan TE, et al. Diet Quality and Colorectal Cancer Risk in the Women's Health Initiative Observational Study. Am J Epidemiol. 2016;184(1):23-32.

16. Park SY, Boushey CJ, Wilkens LR, Haiman CA, Le Marchand L. High-Quality Diets Associate With Reduced Risk of Colorectal Cancer: Analyses of Diet Quality Indexes in the Multiethnic Cohort. Gastroenterology. 2017;153(2):386-94.

17. Stone RAT, Waring ME, Cutrona SL, Kiefe $\mathrm{Cl}$, Allison J, Doubeni CA. The association of dietary quality with colorectal cancer among normal weight, overweight and obese men and women: a prospective longitudinal study in the USA. Bmj Open. 2017;7(6).

18. Cheng E, Um CY, Prizment AE, Lazovich D, Bostick RM. Evolutionary-Concordance Lifestyle and Diet and Mediterranean Diet Pattern Scores and Risk of Incident Colorectal Cancer in lowa Women. Cancer Epidemiol Biomarkers Prev. 2018;27(10):1195-202.

19. Lavalette C, Adjibade M, Srour B, Sellem L, Fiolet T, Hercberg S, et al. Cancer-Specific and General Nutritional Scores and Cancer Risk: Results from the Prospective NutriNet-Sante Cohort. Cancer Res. 2018;78(15):4427-35.

20. Petimar J, Smith-Warner SA, Fung TT, Rosner B, Chan AT, Hu FB, et al. Recommendationbased dietary indexes and risk of colorectal cancer in the Nurses' Health Study and Health Professionals Follow-up Study. Am J Clin Nutr. 2018;108(5):1092-103. 
21. Lee GH, Malietzis G, Askari A, Bernardo D, Al-Hassi HO, Clark SK. Is right-sided colon cancer different to left-sided colorectal cancer? - a systematic review. Eur J Surg Oncol. 2015;41(3):300-8.

22. Paschke S, Jafarov S, Staib L, Kreuser ED, Maulbecker-Armstrong C, Roitman M, et al. Are Colon and Rectal Cancer Two Different Tumor Entities? A Proposal to Abandon the Term Colorectal Cancer. Int J Mol Sci. 2018;19(9).

23. van den Brandt PA, Goldbohm RA, van 't Veer P, Volovics A, Hermus RJ, Sturmans F. A largescale prospective cohort study on diet and cancer in The Netherlands. J Clin Epidemiol. 1990;43(3):28595.

24. van den Brandt PA, Schouten LJ, Goldbohm RA, Dorant E, Hunen PM. Development of a record linkage protocol for use in the Dutch Cancer Registry for Epidemiological Research. Int J Epidemiol. 1990;19(3):553-8.

25. Goldbohm RA, van den Brandt PA, Brants HA, van 't Veer P, Al M, Sturmans F, et al. Validation of a dietary questionnaire used in a large-scale prospective cohort study on diet and cancer. Eur J Clin Nutr. 1994;48(4):253-65.

26. Volovics A, van den Brandt PA. Methods for the analyses of case-cohort studies. Biometrical J. 1997;39(2):195-214.

27. Prentice RL. A case-cohort design for epidemiologic cohort studies and disease prevention trials. Biometrika. 1986;73(1):1-11.

28. Goldbohm RA, van ' $t$ Veer P, van den Brandt PA, van ' $t$ Hof MA, Brants HA, Sturmans F, et al. Reproducibility of a food frequency questionnaire and stability of dietary habits determined from five annually repeated measurements. Eur J Clin Nutr. 1995;49(6):420-9.

29. NEVO table. Dutch food composition table 1986-1987. The Hague, the Netherlands: Voorlichtingsbureau voor de Voeding; 1986.

30. Trichopoulou A, Kouris-Blazos A, Wahlqvist ML, Gnardellis C, Lagiou P, Polychronopoulos E, et al. Diet and overall survival in elderly people. BMJ. 1995;311(7018):1457-60.

31. Trichopoulou A, Costacou T, Bamia C, Trichopoulos D. Adherence to a Mediterranean diet and survival in a Greek population. N Engl J Med. 2003;348(26):2599-608.

32. Fung TT, McCullough ML, Newby PK, Manson JE, Meigs JB, Rifai N, et al. Diet-quality scores and plasma concentrations of markers of inflammation and endothelial dysfunction. Am J Clin Nutr. 2005;82(1):163-73.

33. Mitrou PN, Kipnis V, Thiebaut AC, Reedy J, Subar AF, Wirfalt E, et al. Mediterranean dietary pattern and prediction of all-cause mortality in a US population: results from the NIH-AARP Diet and Health Study. Arch Intern Med. 2007;167(22):2461-8.

34. Fedirko V, Tramacere I, Bagnardi V, Rota M, Scotti L, Islami F, et al. Alcohol drinking and colorectal cancer risk: an overall and dose-response meta-analysis of published studies. Ann Oncol. 2011;22(9):1958-72.

35. World Cancer Research Fund / American Institute for Cancer Research. Continuous update project expert report 2018. Diet, nutrition, physical activity and colorectal cancer. 2018. Available from: https://www.wcrf.org/dietandcancer.

36. Lin DY, Wei LJ. The Robust Inference for the Cox Proportional Hazards Model. J Am Stat Assoc. 1989;84(408):1074-8.

37. Grambsch PM, Therneau TM. Proportional Hazards Tests and Diagnostics Based on Weighted Residuals. Biometrika. 1994;81(3):515-26.

38. Akaike H. A new look at the statistical model identification. IEEE Transactions on automatic control. 1974;AC-19(6):716-23.

39. Wacholder S, Gail MH, Pee D, Brookmeyer R. Alternative Variance and Efficiency Calculations for the Case-Cohort Design. Biometrika. 1989;76(1):117-23.

40. de Vogel S, Bongaerts BW, Wouters KA, Kester AD, Schouten LJ, de Goeij AF, et al. Associations of dietary methyl donor intake with MLH1 promoter hypermethylation and related molecular phenotypes in sporadic colorectal cancer. Carcinogenesis. 2008;29(9):1765-73.

41. World Cancer Research Fund / American Institute for Cancer Research. Food, nutrition, physical activity, and the prevention of cancer: a global perspective. Washington DC: American Institute for Cancer Research; 2007.

42. Romaguera D, Vergnaud AC, Peeters PH, van Gils CH, Chan DS, Ferrari P, et al. Is concordance with World Cancer Research Fund/American Institute for Cancer Research guidelines for cancer 
prevention related to subsequent risk of cancer? Results from the EPIC study. Am J Clin Nutr. 2012;96(1):150-63.

43. Vergnaud AC, Romaguera D, Peeters PH, van Gils CH, Chan DS, Romieu I, et al. Adherence to the World Cancer Research Fund/American Institute for Cancer Research guidelines and risk of death in Europe: results from the European Prospective Investigation into Nutrition and Cancer cohort study. Am J Clin Nutr. 2013;97(5):1107-20.

44. van den Brandt PA, Schulpen M. Mediterranean diet adherence and risk of postmenopausal breast cancer: results of a cohort study and meta-analysis. Int J Cancer. 2017;140(10):2220-31.

45. Schulpen $\mathrm{M}$, van den Brandt PA. Adherence to the Mediterranean diet and risk of lung cancer in the Netherlands Cohort Study. Br J Nutr. 2018;119(6):674-84.

46. Schulpen $M$, Peeters PH, van den Brandt PA. Mediterranean diet adherence and risk of esophageal and gastric cancer subtypes in the Netherlands Cohort Study. Gastric Cancer. 2019;22(4):663-74.

47. Jacques PF, Tucker KL. Are dietary patterns useful for understanding the role of diet in chronic disease? Am J Clin Nutr. 2001;73(1):1-2.

48. Verberne L, Bach-Faig A, Buckland G, Serra-Majem L. Association between the Mediterranean diet and cancer risk: a review of observational studies. Nutr Cancer. 2010;62(7):860-70.

49. Wei EK, Giovannucci E, Wu K, Rosner B, Fuchs CS, Willett WC, et al. Comparison of risk factors for colon and rectal cancer. Int J Cancer. 2004;108(3):433-42.

50. Elferink MAG, Toes-Zoutendijk E, Vink GR, Lansdorp-Vogelaar I, Meijer GA, Dekker E, et al. [National population screening for colorectal carcinoma in the Netherlands: results of the first years since the implementation in 2014]. Ned Tijdschr Geneeskd. 2018;162:D2283 (in Dutch).

51. Slimani N, Fahey M, Welch AA, Wirfalt E, Stripp C, Bergstrom E, et al. Diversity of dietary patterns observed in the European Prospective Investigation into Cancer and Nutrition (EPIC) project. Public Health Nutr. 2002;5(6B):1311-28. 


\section{Supplementary material}

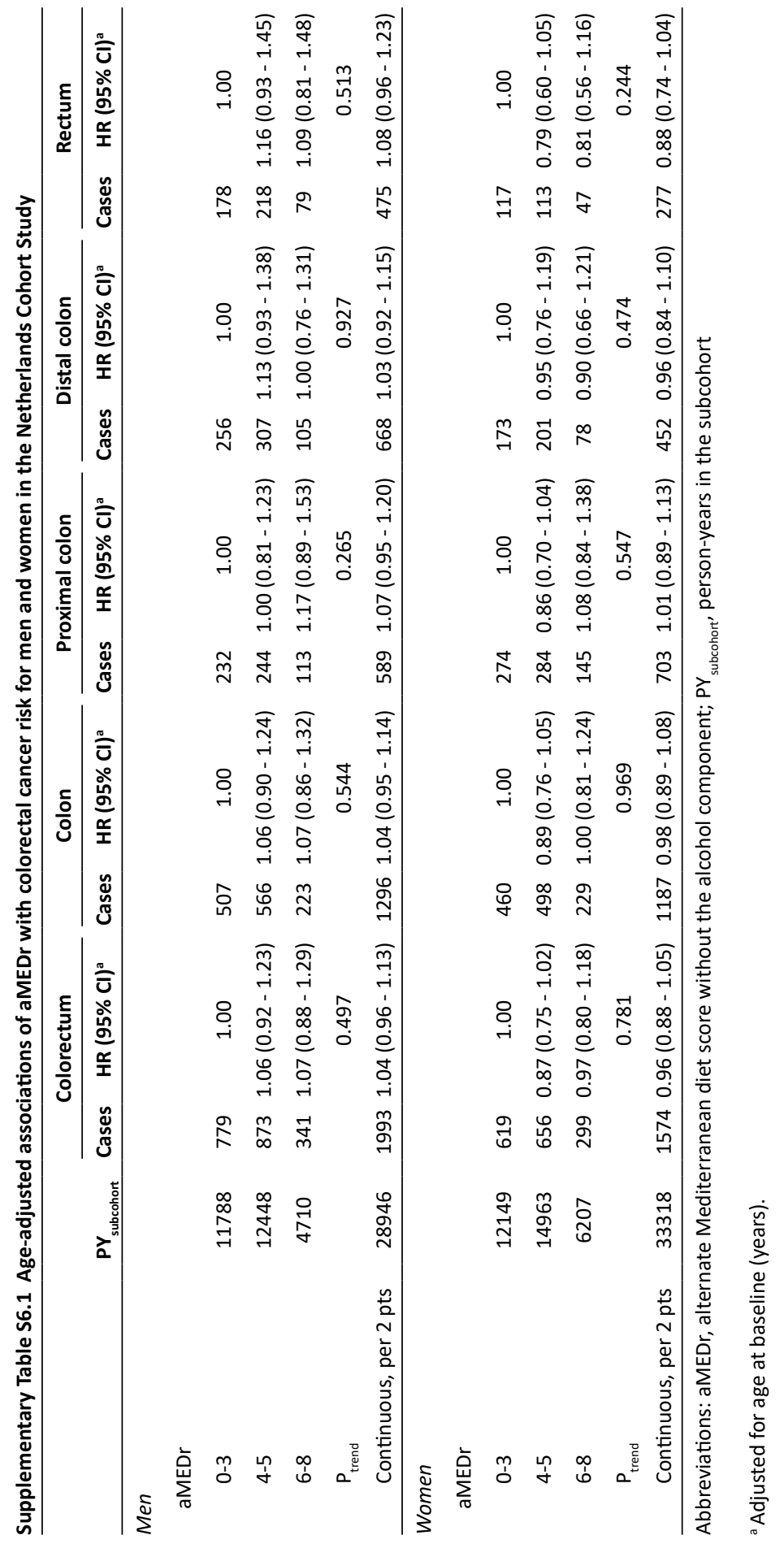


Mediterranean diet and colorectal cancer risk 


\section{Chapter 7}

\section{Adherence to the Mediterranean diet and risks of prostate and bladder cancer in the Netherlands Cohort Study}




\begin{abstract}
Background

Prostate and urinary bladder cancer are frequently occurring cancers with few risk factors identified. We examined the relation of Mediterranean diet (MD) adherence with risks of prostate and bladder cancer in the Netherlands Cohort Study (NLCS).
\end{abstract}

\title{
Methods
}

Data were available for 58279 men and 62573 women, who completed a baseline questionnaire on diet and other cancer risk factors. Multiple MD scores, including the alternate Mediterranean diet score without alcohol (aMEDr), were calculated to assess MD adherence. After 20.3 years of follow-up, 3868 prostate cancer cases (advanced: 1256) and 1884 bladder cancer cases could be included in multivariable Cox proportional hazards analyses.

\section{Results}

aMEDr was not associated with advanced prostate cancer risk [HR $\mathrm{Her}_{\text {po-point increment }}(95 \%$ $\mathrm{Cl})=1.06(0.96-1.17)]$. In contrast, higher aMEDr values were associated with a significantly increased risk of nonadvanced prostate cancer $\left(p_{\text {trend }}=0.04\right)$. For bladder cancer risk, no association was observed with aMEDr $\left[\mathrm{HR}_{\text {per two-point increment }}(95 \% \mathrm{Cl})=1.00(0.92-1.09)\right]$. Absolute scores based on the WCRF/AICR dietary recommendations were not associated with prostate or bladder cancer risk.

\section{Conclusions}

MD adherence, measured by aMEDr or other MD scores, was not associated with decreased risks of advanced prostate cancer and bladder cancer in the NLCS. Higher levels of careseeking behavior, screening attendance and prostate cancer awareness in higher educated men with healthier lifestyles could potentially explain the positive associations that we observed for nonadvanced prostate cancer risk.

\section{Impact}

MD adherence does not seem to reduce the risk of (advanced) prostate cancer or bladder cancer. 


\section{Introduction}

Worldwide, cancers of the prostate and urinary bladder were estimated to be the second and tenth most commonly diagnosed cancer types in 2018 [1]. Together, these cancer types were responsible for over half a million deaths in this year [1]. So far, only advancing age, African-American race, family history of prostate cancer and genetic predisposition have been identified as established risk factors for prostate cancer [2, 3]. Tobacco smoking is the most important risk factor for bladder cancer [4]. Other bladder cancer risk factors include Schistosoma haematobium infection, environmental and occupational exposure to chemicals, and exposure to arsenic in drinking water [5]. The high incidences, slow disease development and progression (prostate cancer), and high recurrence rates (bladder cancer), make prostate and bladder cancer suitable targets for preventive approaches [6-8].

The traditional Mediterranean dietary pattern (MD) is mainly based on plant foods. Intakes of vegetables, legumes, fruits, nuts, whole grains, fish and monounsaturated fatty acids (MUFA, from olive oil) are high in the MD, whereas animal foods (e.g., meats and dairy products) are consumed in limited amounts. Typically, alcohol is consumed in moderation and usually in the form of wine during meals [9-11].

Prostate cancer is a disease with a heterogeneous nature. Advanced and more aggressive prostate tumors may etiologically differ from early, screening-detected forms that otherwise might never have become clinically relevant [2]. Risk factors for prostate cancer subtypes (defined by grade, stage or survival) may differ as they may exert their effect via different biological pathways [12]. Therefore, effects of potential risk factors on advanced prostate cancer risk are of primary interest. Up until now, two prospective cohort studies from the United States (US) have evaluated the relation of a priori defined MD adherence with advanced prostate cancer risk and did not observe an association [13, 14]. In contrast to the results for advanced prostate cancer, the prospective evidence suggests that MD adherence might be associated with a reduced risk of (invasive) bladder cancer/urothelial cell carcinoma (UCC) $[15,16]$. However, the inverse associations were not statistically significant.

In this analysis of the Netherlands Cohort Study (NLCS), we examined associations between a priori defined MD adherence and risks of prostate and urinary bladder cancer. Associations were compared for subtypes of the investigated cancer sites classified by stage at diagnosis (prostate cancer) or malignancy grade (bladder cancer). In addition, the effect of exclusion of alcohol from the MD scores was evaluated and performances of the relative MD scores were compared to those of absolute scores based on the dietary recommendations to prevent cancer issued by the World Cancer Research Fund/American Institute for Cancer Research (WCRF/AICR).

\section{Materials and methods}

\section{Study population and cancer follow-up}

The prospective NLCS includes 58279 men and 62573 women, aged 55 to 69 years [1720]. Study participants consented to participate by completing a self-administered baseline questionnaire on diet and other cancer risk factors in September 1986. A case-cohort approach was applied to process and analyze the data efficiently [17, 20, 21]. A subcohort $(\mathrm{N}=5000)$ was randomly drawn just after baseline to estimate accumulated person-years at risk, whereas cases originated from the entire cohort. Subcohort members were followed 
up biennially for vital status. The NLCS was approved by the institutional review boards from Maastricht University and the Netherlands Organization for Applied Scientific Research, and was conducted in accordance with the Declaration of Helsinki.

Incident cancer cases were detected annually for 20.3 years of follow-up (baseline until 31 December 2006) through record linkage with the Netherlands Cancer Registry and the nationwide Dutch Pathology Registry (PALGA) [18]. In total, 3978 prostate cancer cases (ICD-O-3 code C61), 2049 bladder cancer cases (ICD-O-3 code C67) and 4084 subcohort members (men: 2057, women: 2027) were eligible for inclusion in the present analyses (Supplementary Figures S7.1 and S7.2). Prostate cancer cases were classified as nonadvanced $(\mathrm{N}=2397$, stages $\mathrm{T} 1 / \mathrm{T} 2, \mathrm{~N} 0$ and $\mathrm{M0})$ or advanced $(\mathrm{N}=1294$, stages $\mathrm{T} 3 / \mathrm{T} 4$ or $\mathrm{N}+$ or $\mathrm{M} 1$ ) at diagnosis, whereas bladder cancer cases were categorized in noninvasive $(N=1053$, malignancy grade 2 ) and invasive ( $\mathrm{N}=996$, malignancy grade 3 ) subtypes. All included cases were microscopically confirmed. Furthermore, eligible cases and subcohort members did not have prevalent cancer at baseline (except skin cancer), and had complete and consistent data available on diet, alcohol and MD adherence.

\section{Exposure assessment}

At baseline, participants were asked about their usual dietary intake during the previous year via a validated, 150-item, semi-quantitative food frequency questionnaire (FFQ) $[19,22]$. Nutrient intakes were derived from the FFQ data utilizing the 1986 Dutch food composition (NEVO) table [23].

\section{Mediterranean diet adherence}

The alternate and modified Mediterranean diet scores (aMED and MMED, respectively) were calculated to estimate the relative level of MD adherence [24-26]. These scores are adaptations of the traditional Mediterranean diet score (tMED) created by Trichopoulou et al. $[27,28]$ and are each composed of nine dietary components. For aMED [24, 26], 1 point (and 0 otherwise) is assigned to mean daily intakes at or above the sex-specific median of vegetables (excluding potatoes), legumes, fruits, nuts, whole grains, fish and the ratio of MUFA to saturated fatty acids (SFA). Inverse scoring is applied to red and processed meats. Finally, 1 point can be obtained for a moderate alcohol consumption, defined as 5-25 g/day $[24,26]$. mMED [25] differs from aMED as follows: fruits and nuts are combined, total intakes of cereal and meat are considered, dairy intake is included (1 point if below sex-specific median), and the ratio of unsaturated fatty acids (MUFA + polyunsaturated fatty acids) to SFA replaces the MUFA:SFA ratio. Furthermore, other cut-offs are used to define moderate alcohol consumption (men: 10-50 g/day, women: 5-25 g/day) [25]. Before calculation of the MD scores, food intakes were standardized to daily energy intakes of 2000 (women) and 2500 (men) kilocalories [26, 27]. aMED and mMED range from 0 to 9 points (lowest to highest MD adherence). Because alcohol consumption has been associated with increased risks of several types of cancer [29], we also created aMED and MMED variants without alcohol (aMEDr and mMEDr, respectively) ranging from 0 to 8 points. 


\section{Statistical analyses}

We evaluated relations between MD adherence and risks of prostate and bladder cancer (subtypes) using Cox proportional hazards models with duration of follow-up as time scale to estimate hazard ratios (HRs) and $95 \%$ confidence intervals $(95 \% \mathrm{Cls})$. Person-years at risk in the subcohort were calculated from baseline until prostate or bladder cancer diagnosis, death, emigration, loss to follow-up or end of follow-up, whichever came first. Sampling from the cohort introduces additional variance. Therefore, standard errors were calculated using the Huber-White sandwich estimator [30]. The proportional hazards assumption was checked by scaled Schoenfeld residuals tests and visual inspection of $-\ln (-\ln )$ survival plots [31].

We tested associations of MD adherence with risks of prostate and bladder cancer (subtypes) in age- (and sex-)adjusted and fully adjusted analyses, in which MD scores were modelled both categorically [low: 0-3, middle: 4-5, high: 6-8(9)] and continuously (per two-point increment) $[25,26]$. Men and women were combined in the models for bladder cancer, because there was no statistically significant interaction by sex. The fully adjusted models concerning prostate cancer risk were adjusted for the following predefined confounders: age at baseline, body mass index (BMI), alcohol consumption (except for models containing MD scores including alcohol), total daily energy intake, highest level of education and family history of prostate cancer. For bladder cancer risk, sex and cigarette smoking behavior (status, frequency and duration) were also listed as predefined confounders. Additionally, these analyses were adjusted for family history of bladder cancer instead of prostate cancer. Other confounders considered, but not included (removal resulted in $<10 \%$ change in the effect estimate of the MD score), were cigarette smoking status (prostate cancer only), non-occupational physical activity, history of diabetes, height (prostate cancer only), tea consumption and coffee consumption (bladder cancer only). Sex-specific median MD score values in the subcohort were appointed to each adherence category and fitted continuously in Cox regression models to perform trend tests. A competing risks procedure was applied to test for heterogeneity across the prostate and bladder cancer subtypes [32]. Standard errors for the observed differences were estimated using a bootstrapping method specifically designed for the case-cohort approach [33].

Model fits of the various MD scores considered (aMEDr and mMEDr, with and without alcohol) were compared using Akaike's Information Criterion (AIC) [34]. Because of the equal or better performance of aMEDr compared to mMEDr in both the current and previous NLCS analyses [35-37], the Results section of this article mainly focuses on associations with aMEDr and subgroup analyses were only performed using this score. We preferred the aMED variant without alcohol (aMEDr), because alcohol consumption is a risk factor for several types of cancer [29].

Potential effect modification by sex (bladder cancer only), cigarette smoking status (bladder cancer only), alcohol consumption, BMI, educational level and family history of prostate/ bladder cancer was explored by testing the statistical significance of interaction terms between these factors and aMEDr. Additionally, HRs were estimated for strata of the potential effect modifying factors. For prostate cancer risk, we estimated associations with the MD scores within time periods before (1986-1994) and after (1995-2006) the introduction of prostate-specific antigen (PSA) testing in clinical practice in the Netherlands. Furthermore, the effect of excluding the first two years of follow-up was evaluated.

Because of the use of cohort-specific cut-offs, the MD scores used measure relative levels 
of MD adherence. Therefore, we also evaluated associations of prostate and bladder cancer (subtypes) with an absolute score based on the dietary part of the 2007 cancer prevention recommendations published by the WCRF/AICR [38]. The WCRF/AICR score used in the current study includes the recommendations concerning intakes of foods and drinks that promote weight gain, plant foods, red and processed meats, alcohol and salt. When possible, recommendations were operationalized as in the European Prospective Investigation into Cancer and Nutrition (EPIC) cohort $[39,40]$. For a detailed description of the scoring method and the absolute cut-offs used we refer to a previous NLCS article [35]. The resulting sum score ranged from 0 to 5 points (lowest to highest adherence). Additionally, we created a variant of the WCRF/AICR score without the alcohol recommendation that ranged from 0 to 4 points. Fully adjusted $\mathrm{HRs}$ and $95 \% \mathrm{Cl}$ sere estimated per standard deviation (SD) increase in WCRF/AICR score. For comparison, we also estimated HRs and 95\% Cls per SD-increment for the aMED variables (with and without alcohol). Finally, AIC was used to compare model fits of the WCRF/AICR score and aMED variables. All analyses were performed using Stata version 15 . Two-sided $p$-values $<0.05$ were considered statistically significant.

\section{Results}

\section{Baseline characteristics NLCS subcohort and cases of prostate and bladder cancer}

Table 7.1 compares baseline characteristics of subcohort members with those of prostate and bladder cancer cases. The mean level of MD adherence was similar for cases and subcohort members, except for the slightly lower mean aMEDr value in female noninvasive bladder cancer cases. Compared to subcohort members, prostate cancer cases were less often current smokers, higher educated (particularly nonadvanced prostate cancer), more physically active (particularly nonadvanced prostate cancer) and more likely to have a family history of prostate cancer. When comparing nonadvanced with advanced cases at diagnosis, nonadvanced cases more frequently had a high level of education and were slightly more active. Bladder cancer cases were more often current smokers, consumed higher amounts of alcohol and reported more commonly a family history of bladder cancer than subcohort members. Patterns were mostly comparable for noninvasive and invasive bladder cancer cases.

\section{Mediterranean diet adherence and risks of prostate and bladder cancer}

Fully adjusted associations of MD adherence with prostate and bladder cancer incidence are presented in Tables 7.2 and 7.3, respectively. For age- (and sex-)adjusted associations we refer to Supplementary Tables S7.1 (prostate) and S7.2 (bladder). The number of subjects included in the Cox models is slightly smaller than the number of eligible participants due to missing values in covariates.

\section{Prostate cancer}

Higher MD adherence, measured by aMEDr, was associated with an increased risk of prostate cancer $\left[\mathrm{HR}_{\text {per two-point increment }}(95 \% \mathrm{Cl}): 1.09\right.$ (1.01 - 1.17)] (Table 7.2). Though, this positive association was mainly present in nonadvanced cases. For nonadvanced prostate cancer risk, the HR $(95 \% \mathrm{Cl})$ comparing high to low aMEDr values was 1.22 (1.01 - 1.48) with a significant test for trend $(p=0.04)$. However, aMEDr was not significantly associated 


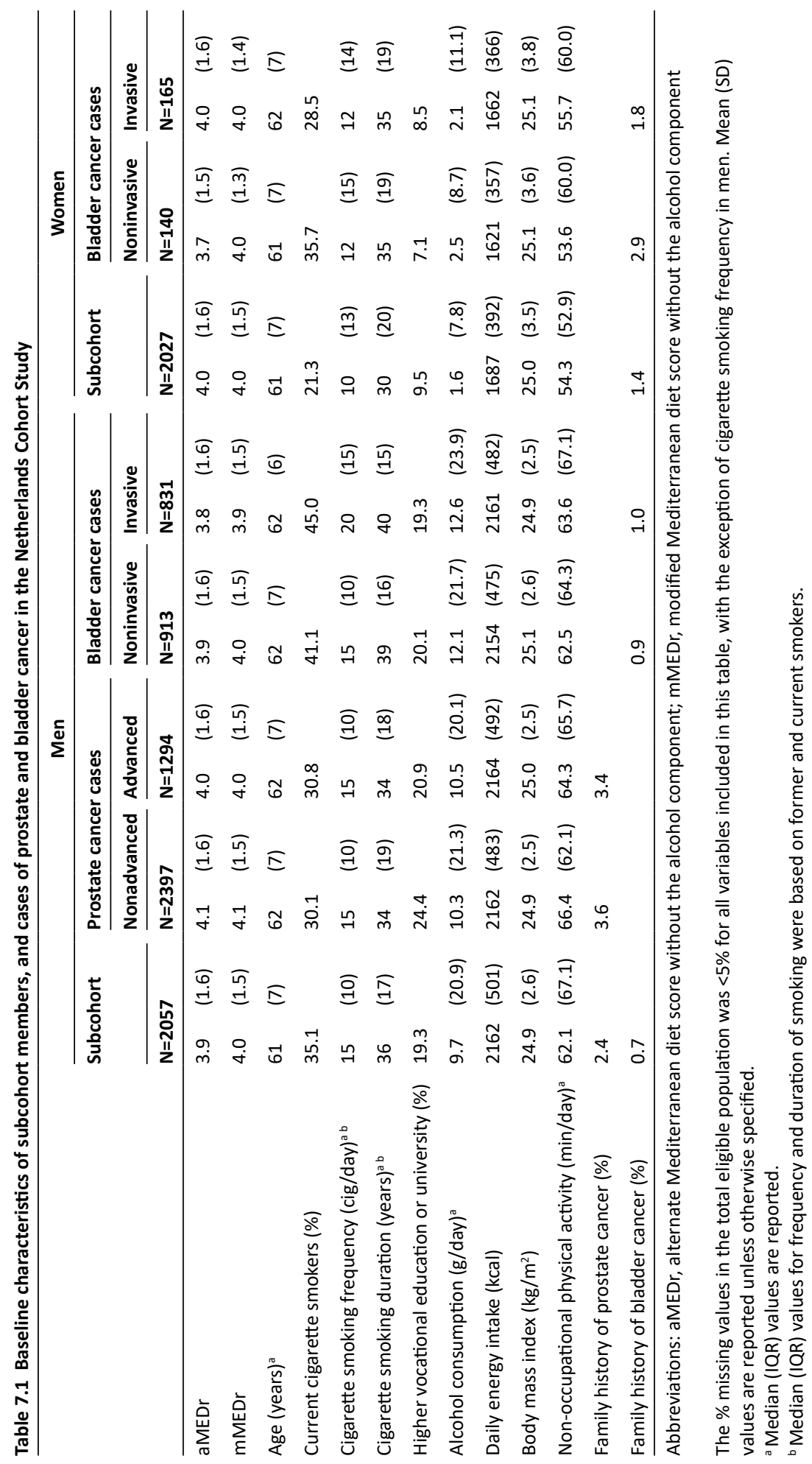




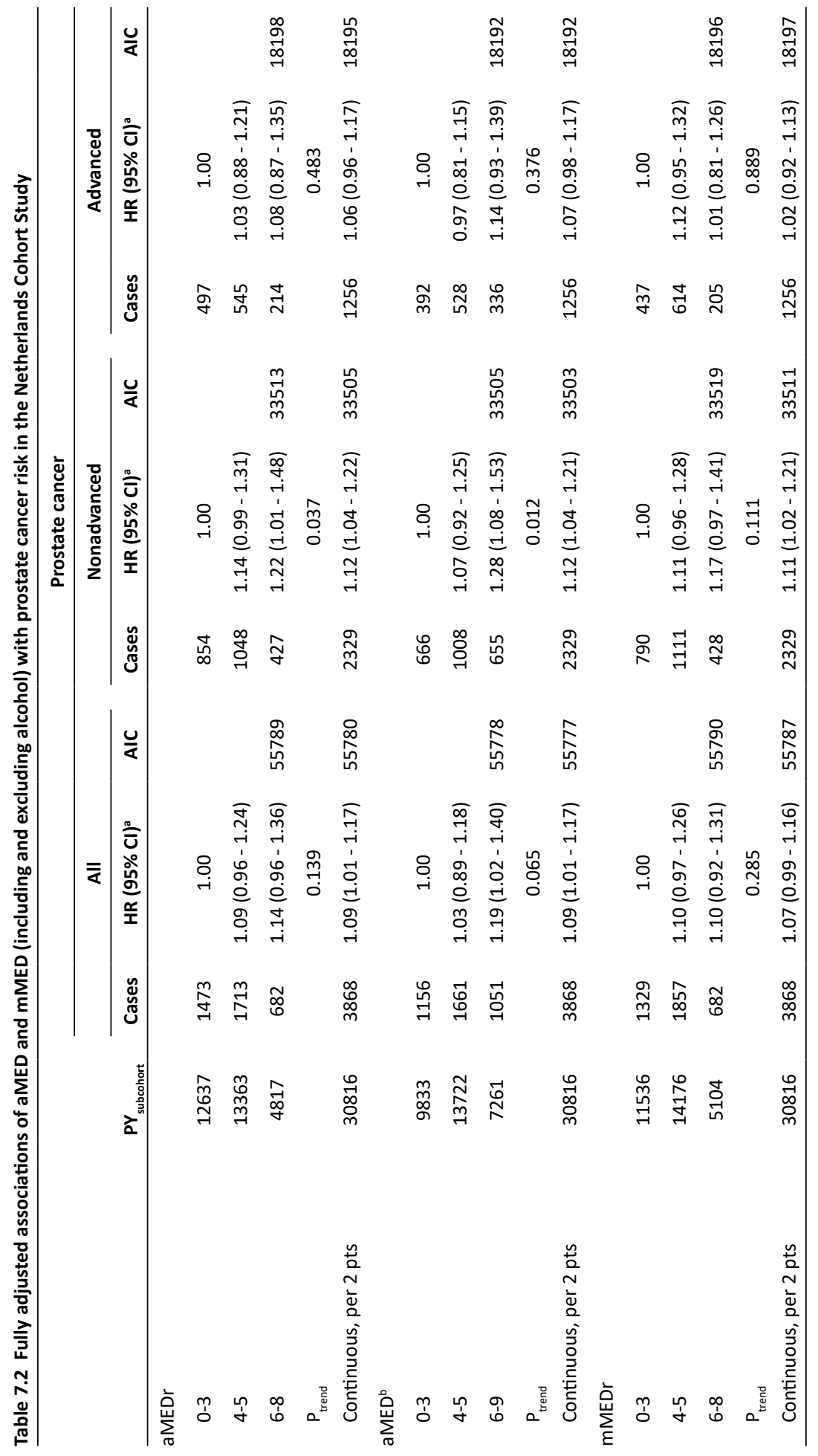




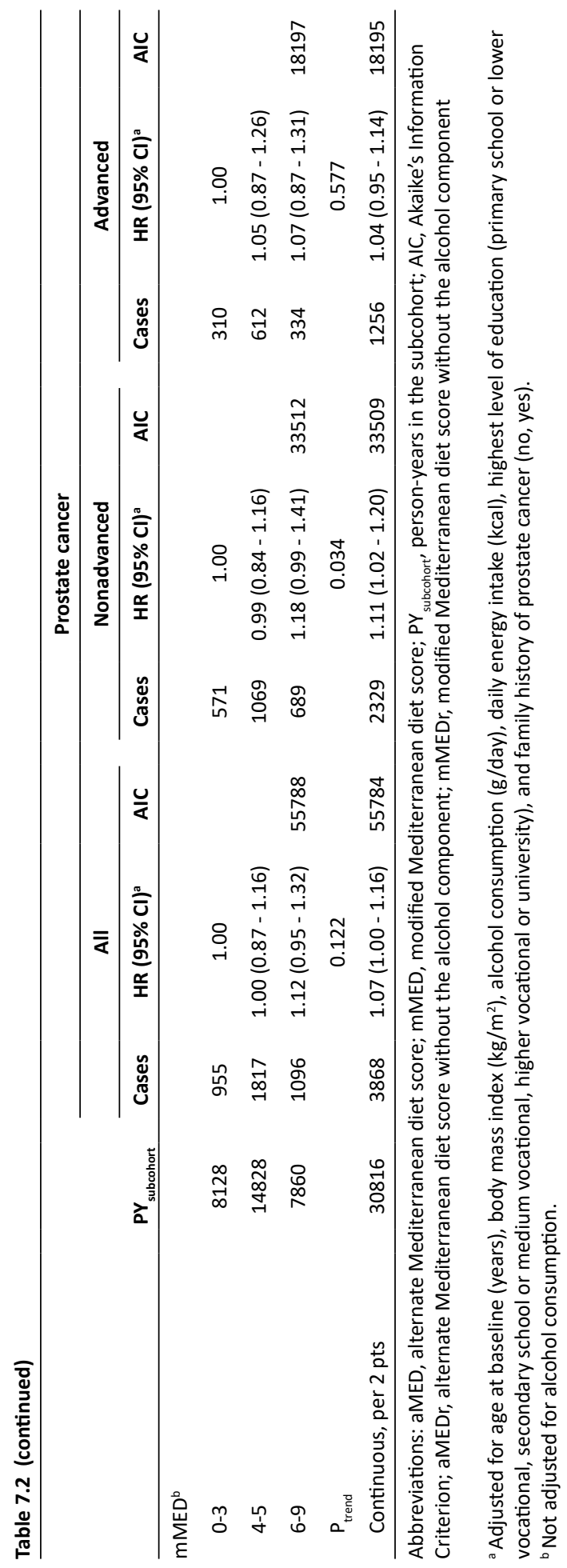




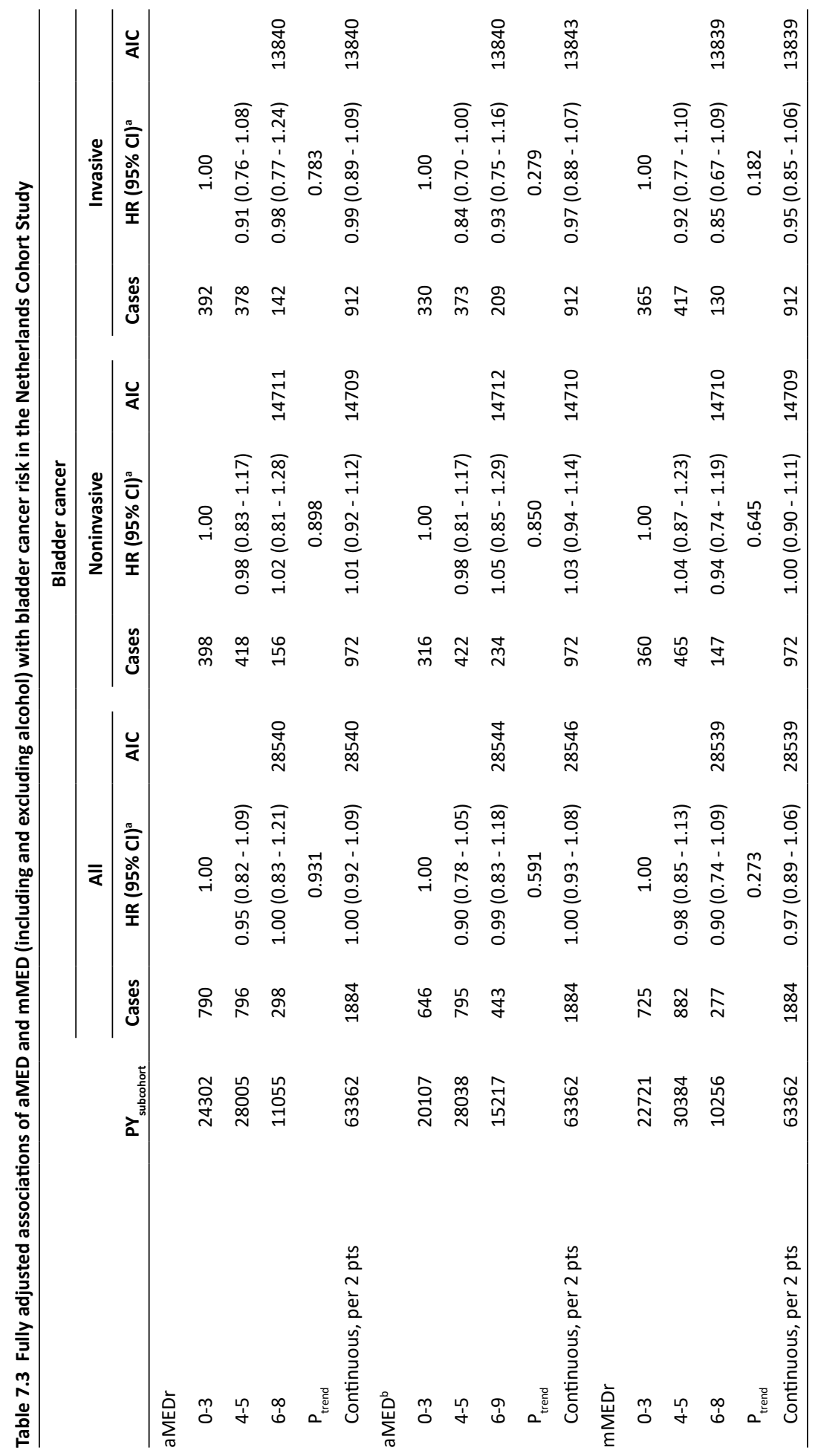




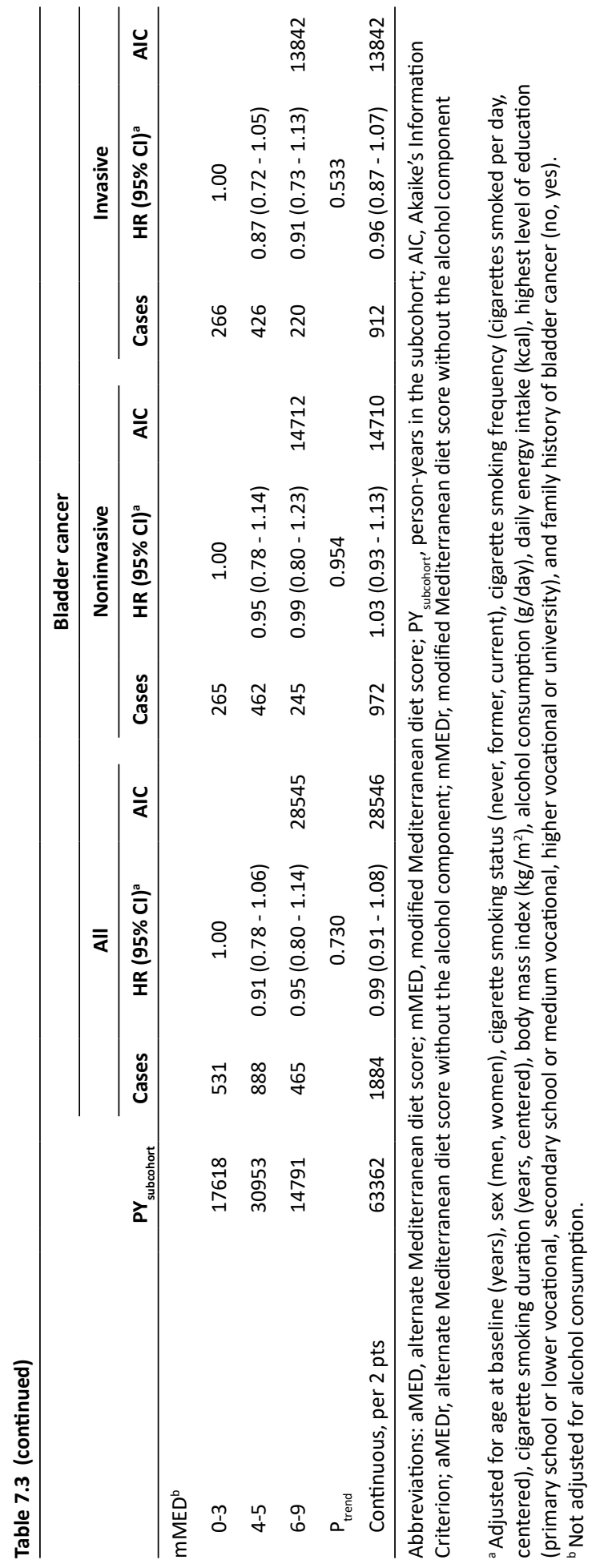


with the risk of advanced prostate cancer $\left[\mathrm{HR}_{\text {per two-point increment }}(95 \% \mathrm{Cl}): 1.06(0.96-1.17)\right]$. Despite this difference between the prostate cancer subtypes, tests for heterogeneity were not statistically significant. Associations of similar directions were observed when MD adherence was assessed using mMEDr and inclusion of alcohol in the MD scores did not notably change the results. Comparison of model performances showed equal or better (nonadvanced prostate cancer) fits for aMEDr compared to mMEDr (Table 7.2). Additionally, model fits were generally better for scores with alcohol than scores without alcohol.

Associations of aMEDr with risks of nonadvanced and advanced prostate cancer did not significantly differ across strata of potential effect modifiers (Table 7.4). Nevertheless, increasing aMEDr was associated with a significantly increased risk of both prostate cancer subtypes among men in the highest education category, whereas there was no clear evidence of an association in the other education categories. Results were comparable after exclusion of the first two years of follow-up [HR per two-point increment in aMEDr $: 1.09$ (total), 1.13 (nonadvanced), 1.06 (advanced)]. Additionally, the strength of the associations did not significantly differ before (1986-1994) and after (1995-2006) the introduction of PSA testing in clinical practice in the Netherlands (Table 7.4). However, the positive association between aMEDr and nonadvanced prostate cancer risk was only statistically significant in the late period (1995-2006).

\section{Bladder cancer}

MD adherence was not significantly associated with bladder cancer risk, regardless of the MD score used (Table 7.3). HRs (95\% Cls) per two-point increase in aMEDr were 1.00 (0.92 - 1.09), $1.01(0.92-1.12)$ and $0.99(0.89-1.09)$ for total, noninvasive and invasive bladder cancer, respectively. In contrast to the fully adjusted analyses, inverse trends (not always significant) seemed to be present between MD adherence and risks of total and invasive bladder cancer in age- and sex-adjusted analyses ( $p=0.04$ for mMED and invasive bladder cancer, Supplementary Table S7.2). Comparable performances were observed for models containing aMEDr and mMEDr (Table 7.3). Furthermore, MD scores without alcohol fitted equally or better than their equivalents including alcohol.

There was no evidence of effect modification by sex, cigarette smoking status, alcohol consumption, educational level and family history of bladder cancer (Table 7.5). Higher aMEDr values seemed to be associated with a non-significantly decreased risk of bladder cancer in subjects with a normal BMI, but not in overweight or obese subjects $\left[p_{\text {interaction }}\right.$ : 0.026 (total), 0.037 (noninvasive), 0.128 (invasive)]. Associations did not essentially change

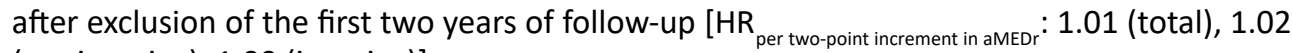
(noninvasive), 1.00 (invasive)].

\section{Dietary WCRF/AICR recommendations and risks of prostate and bladder cancer}

Because values of aMED indices are population-dependent, we compared these indices to absolute WCRF/AICR scores (Table 7.6). The WCRF/AICR scores were not significantly associated with prostate and bladder cancer risk, but as with the aMED indices, associations with prostate cancer risk were in the positive direction. For prostate cancer risk, WCRF/AICR scores had worse model fits compared to aMED indices, particularly when considering the nonadvanced subtype (Supplementary Table S7.3). Comparable model performances were observed for bladder cancer risk (Supplementary Table S7.3). 


\section{Discussion}

In the large prospective NLCS, a priori defined MD adherence (aMEDr) was associated with a significantly increased risk of nonadvanced prostate cancer. In contrast, no association was observed with advanced prostate cancer risk. MD adherence was not associated with risks of total, noninvasive and invasive bladder cancer. Model fits were equal or better for aMEDr compared to mMEDr. Additionally, inclusion of alcohol in the MD scores resulted in generally better model fits for prostate cancer risk, whereas the opposite was observed for bladder cancer risk. Finally, adherence to the dietary WCRF/AICR recommendations was not associated with risks of both prostate and bladder cancer.

Previously conducted cohort studies in the US and Europe consistently found no association between a priori defined MD adherence and prostate cancer risk [13, 14, 41, 42]. Similar results were obtained when focusing on advanced cases of prostate cancer specifically [13, 14]. Case-control studies showed less consistent results. One study observed a significant inverse association between a priori defined MD adherence and prostate cancer risk, whereas no relation was present in another study $[43,44]$. The vulnerability of the case-control design to several types of bias, including recall and selection biases, could potentially explain this inconsistency. Furthermore, prospective cohort studies may also have some limitations. For example, reliance on a single assessment of dietary intake at baseline may lead to exposure misclassification and attenuated associations. Although exposure misclassification could have contributed to the null findings of the previously conducted cohort studies, some cohorts did have updated dietary information available during follow-up [14, 42].

Results of the present study were partially in concordance with results of previous cohort studies. We found higher MD adherence to be significantly associated with an increased risk of nonadvanced prostate cancer, while there was no evidence of an association with advanced prostate cancer risk. Prostate cancer is a heterogeneous disease with potentially etiologically different subtypes that may differ in risk factors $[2,12,45]$. The subgroup of nonadvanced cancers at diagnosis mainly encompasses relatively nonaggressive forms of prostate cancer that progress slowly and might never have become clinically relevant. Approximately half of the diagnosed prostate cancers were estimated to not have caused any harm if they had remained undiagnosed and untreated [45]. The prevalence of undiagnosed prostate cancer in elderly men is high, in $47.3 \%$ of US White and European men above the age of 80 years incident prostate cancer was detected at autopsy [45]. Due to the often indolent nature of nonadvanced prostate cancers, advanced prostate cancer is the subtype of our primary interest. In this study, MD adherence was not significantly associated with advanced prostate cancer risk.

The significant positive associations that we observed between MD adherence and nonadvanced prostate cancer risk could potentially be explained by differences in careseeking behavior, screening attendance and prostate cancer awareness related to education and lifestyle. Male NLCS subcohort members with higher MD adherence overall seemed to have a healthier lifestyle judged by lower levels of smoking and alcohol consumption, and higher levels of physical activity, and were higher educated [36]. Higher educated men with a more health-conscious lifestyle may be more aware of prostate cancer and more prone to seek care or attend screenings, resulting in a larger number of nonadvanced prostate cancer diagnoses in this group, part of which never would have become clinically relevant and otherwise would have remained undiagnosed. Nonadvanced prostate cancer cases in our study were more physically active and higher educated than advanced cases and subcohort 


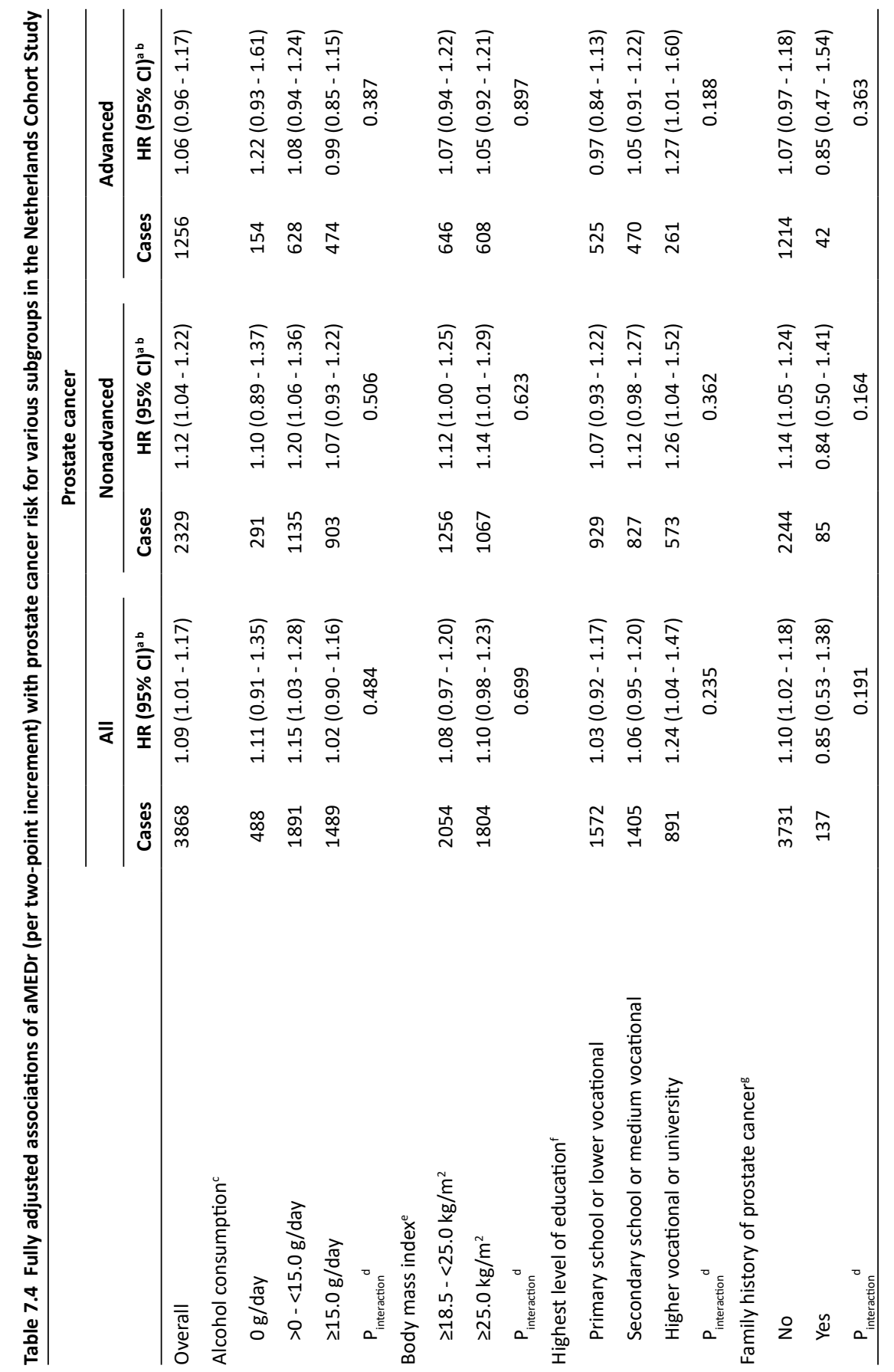




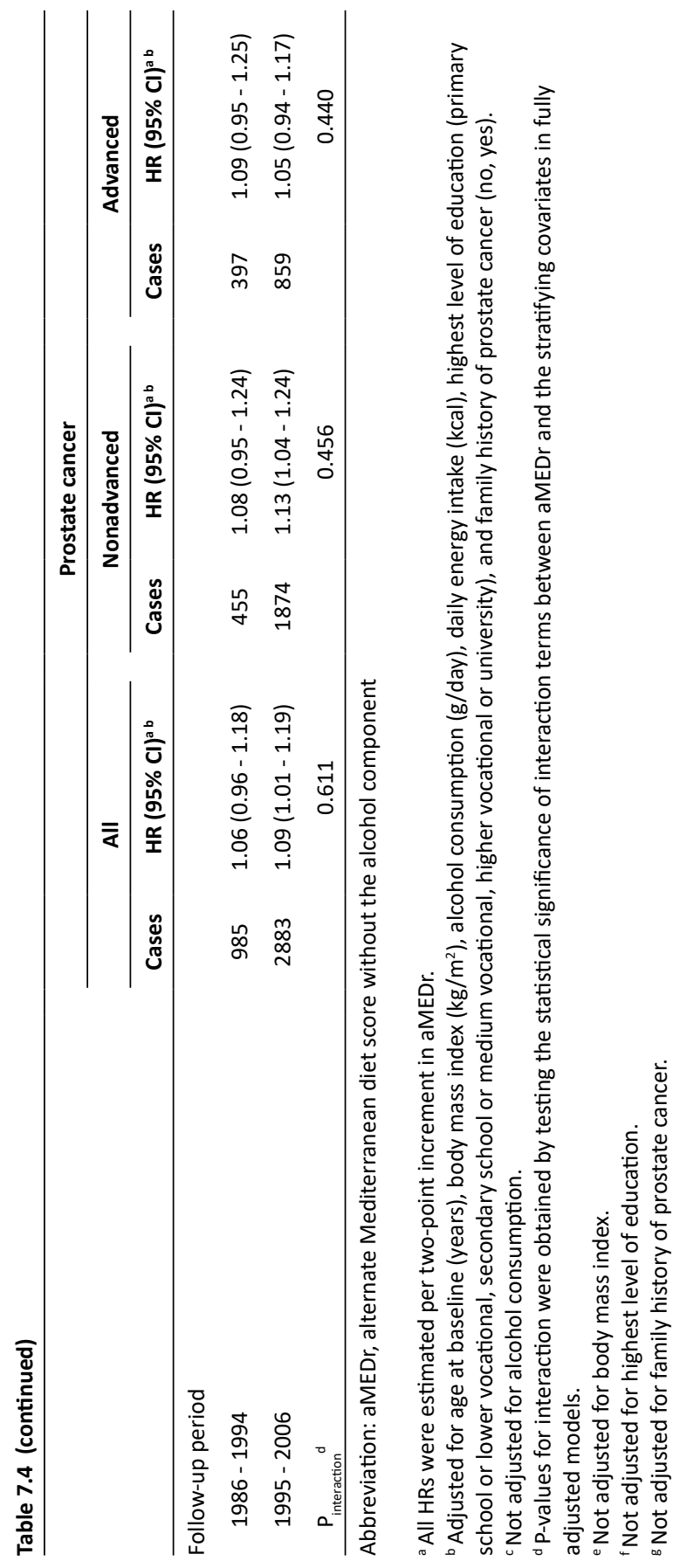




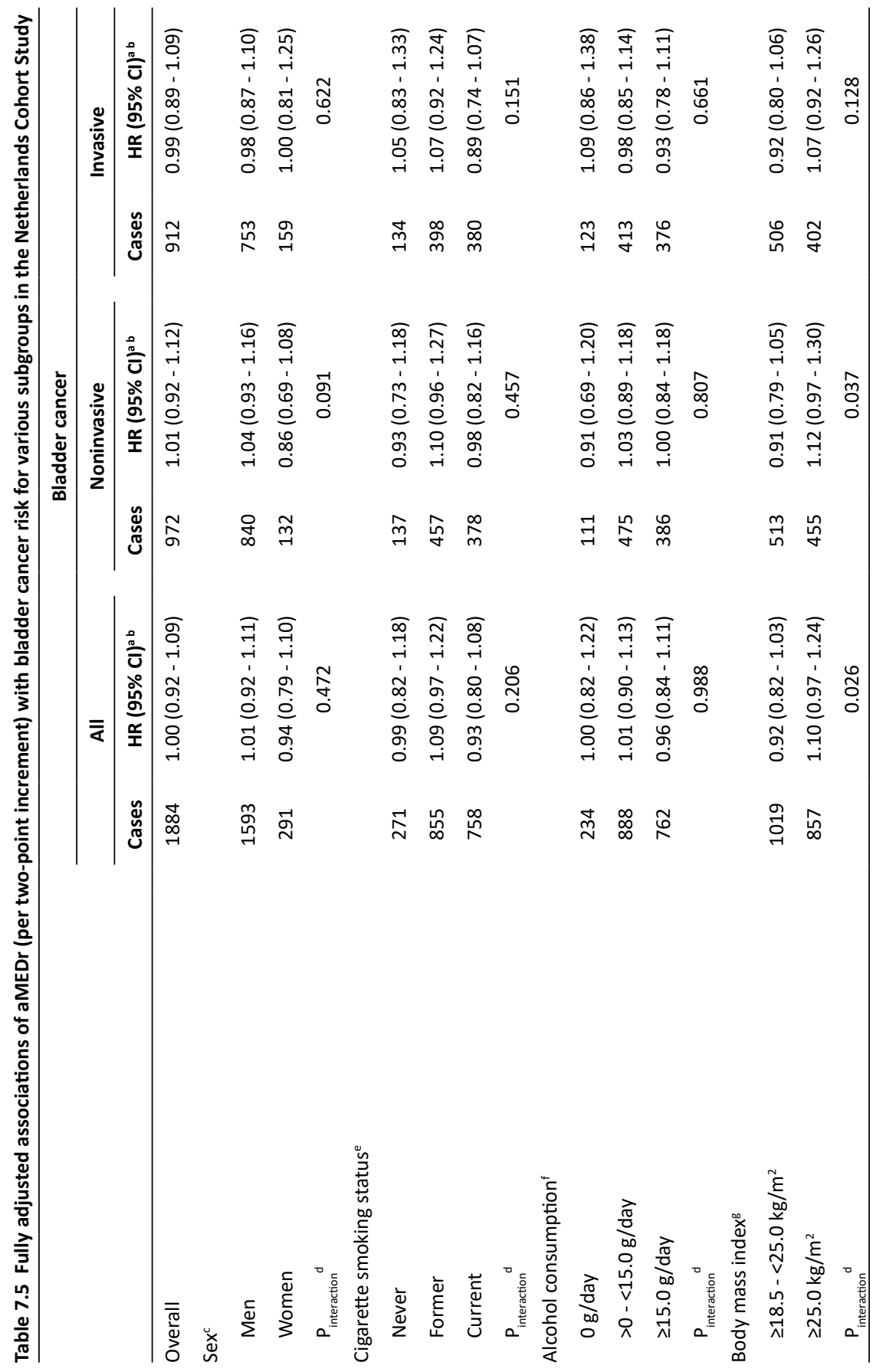




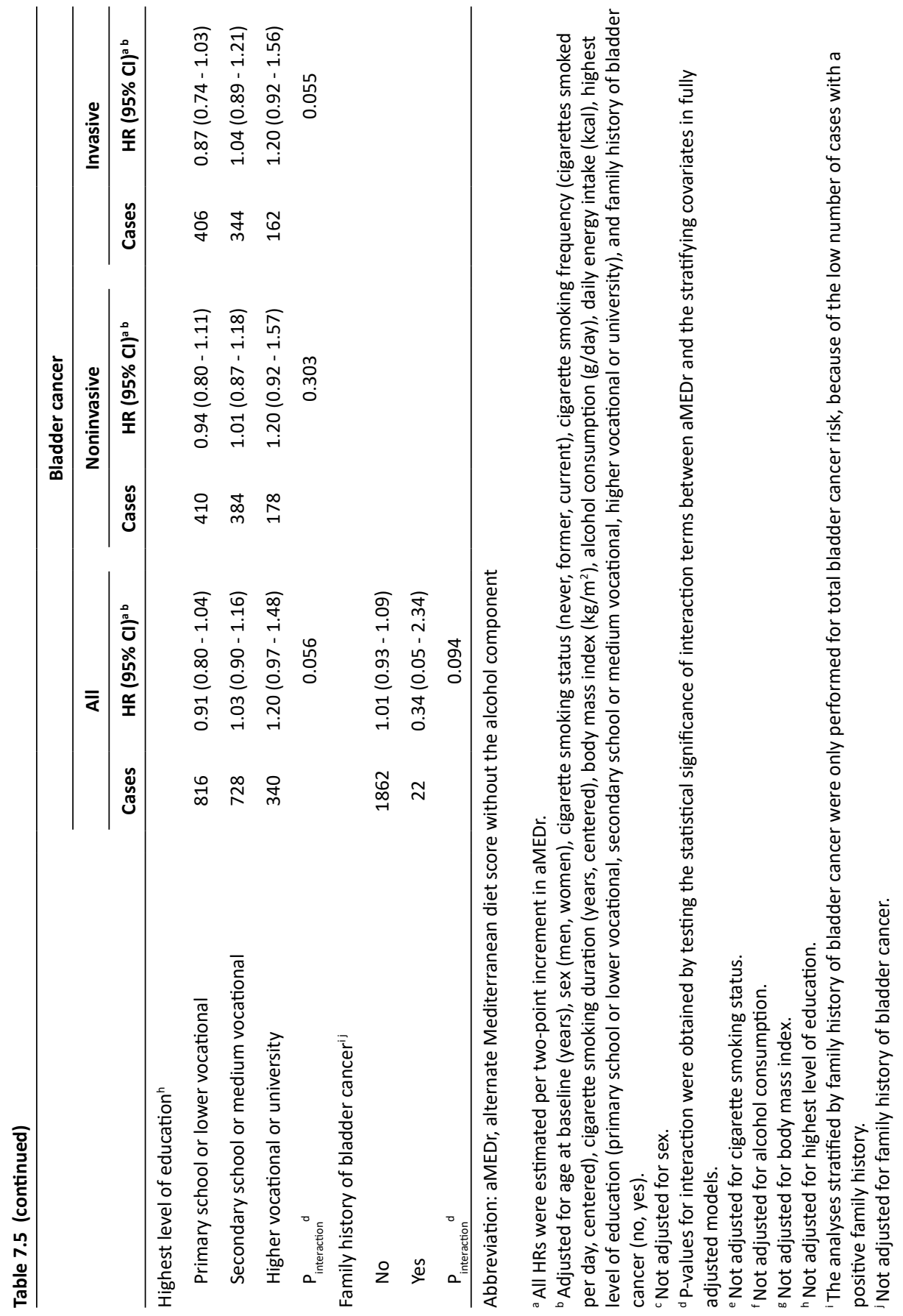




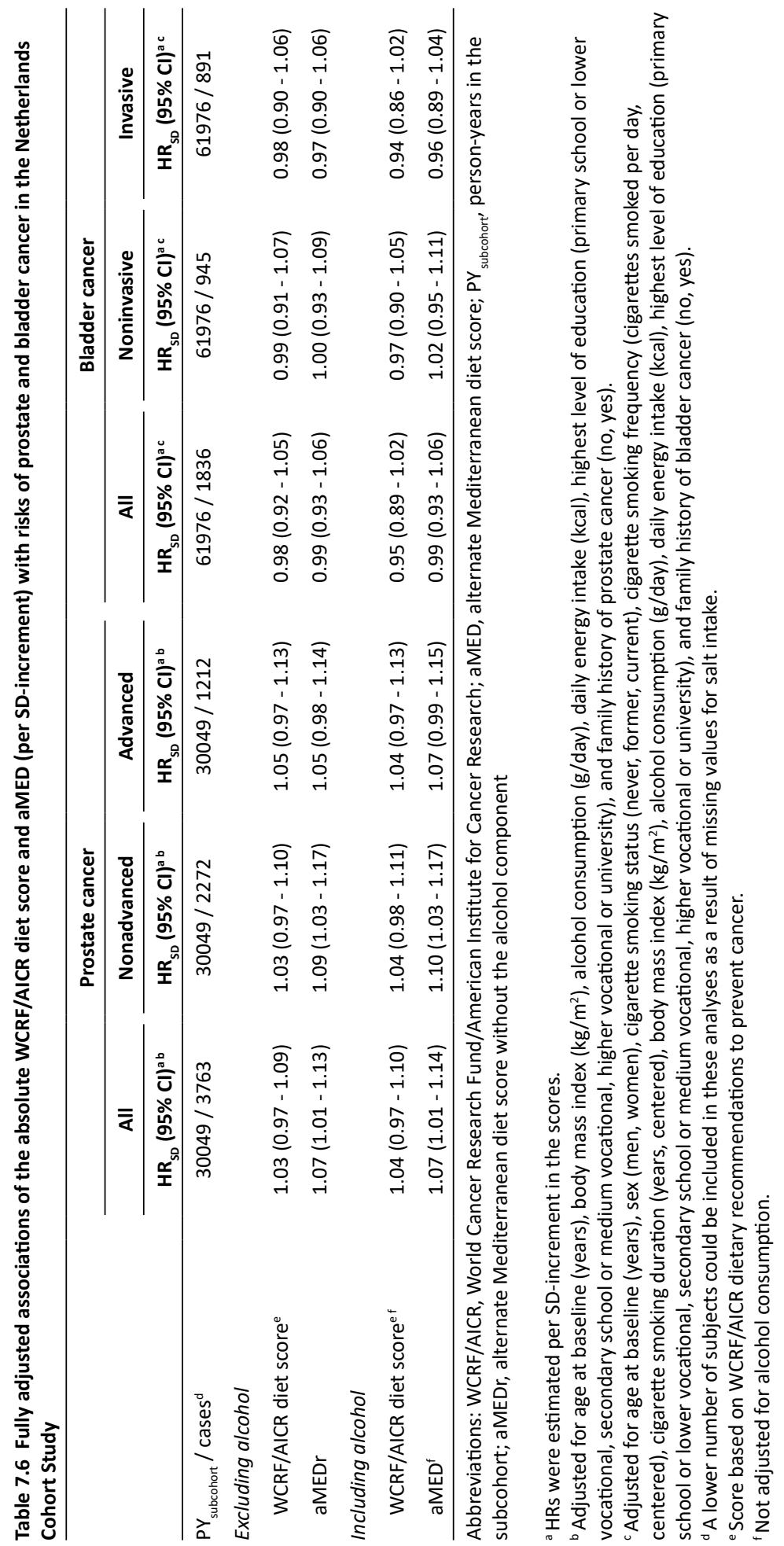


members, implying a healthier lifestyle. Furthermore, the positive association between MD adherence and nonadvanced prostate cancer risk was strongest and only statistically significant in the highest education category, which fits our hypothesis that nonadvanced prostate tumors are more likely to be detected in highly educated men. Finally, results of our stratified analyses by follow-up period showed that the positive association between MD adherence and nonadvanced prostate cancer risk only reached statistical significance in the period after the introduction of PSA testing in clinical practice in the Netherlands (1995-2006).

Similar to our findings for bladder cancer risk, a priori defined MD adherence was not significantly associated with total UCC risk in the prospective EPIC and Melbourne Collaborative Cohort Study (MCCS) cohorts $[15,16]$. Although not statistically significant, HR estimates for risks of total, nonaggressive and aggressive UCC in EPIC were suggestive of an inverse association with MD adherence [15]. Additionally, higher MD adherence seemed to be associated with a decreased risk of invasive UCC $(p=0.06)$, but not superficial UCC, in the Australian MCCS [16]. HR estimates in our study were much closer to unity. Residual confounding by cigarette smoking, which is the most important bladder cancer risk factor [4], could (partially) explain the non-significant inverse associations between MD adherence and (invasive) UCC risk that were observed in the previously conducted prospective studies. The adjustment for cigarette smoking seemed to be less comprehensive in these studies compared to the present study and the inverse associations were restricted to (former and) current smokers. Besides, we also observed (non-significant) inverse associations with invasive bladder cancer risk when we only adjusted our models for age and sex. Very recently, a pooled analysis of 13 prospective cohorts showed that MD adherence was associated with a significantly decreased risk of total, non-muscle-invasive and muscle-invasive bladder cancer [46]. However, this study did not sufficiently adjust for smoking behavior. Finally, an Italian hospital-based case-control study found higher MD adherence to be associated with a significantly decreased UCC risk [47].

In this study, BMI significantly modified the association between MD adherence and risks of total and noninvasive bladder cancer, with non-significant inverse associations being observed among subjects with a normal BMI, but not among overweight or obese subjects. An inverse association has been suggested between BMI and levels of urinary 8-hydroxydeoxyguanosine, a marker of oxidative DNA damage, particularly in smokers [48]. Therefore, subjects with a normal BMI potentially benefit most from the high antioxidant content (e.g., vitamins and polyphenols) of the $\operatorname{MD}[49,50]$. However, this interaction could also be a chance finding. The interaction with BMI was not detected in the EPIC cohort [15] and requires attention in future research. The association of MD adherence with bladder cancer risk did not significantly differ across strata of other potential effect modifiers including sex and smoking status.

Major strengths of the NLCS are its prospective design and the nearly complete follow-up of 20.3 years. The large number of prostate and bladder cancer cases allowed subtypespecific analyses based on tumor stage/invasiveness at diagnosis, extensive adjustment for confounding and stratified analyses for potential effect modifying factors. Despite the comprehensive adjustment for cigarette smoking habits in the analyses concerning bladder cancer risk, residual confounding by smoking (bladder cancer) or unmeasured factors (prostate and bladder cancer) could still have affected our results. For example, we had no information about PSA testing. Nevertheless, associations of MD adherence with prostate cancer risk did not statistically significantly differ in time periods before and after 
the introduction of PSA testing in the Netherlands, making a relevant effect on our results unlikely. Moreover, we were not able to adjust the analyses concerning bladder cancer risk for environmental and occupational exposures to chemicals or exposure to arsenic in drinking water. A final strength of our study includes the high quality of the dietary data. The single baseline measurement of the NLCS-FFQ was shown to perform adequately when compared to nine-day dietary records and dietary habits were reproducible for over at least five years $[19,22]$. However, changes in dietary habits and confounding factors after baseline as well as measurement errors may have attenuated associations. The populationdependent assignment of scores is a weakness of the MD scores that we used to assess MD adherence. Therefore, high MD score values may not necessarily represent a truly Mediterranean way of eating, especially in the Netherlands and other non-Mediterranean countries. Nonetheless, largely similar results were obtained when we used absolute scores based on the WCRF/AICR dietary recommendations to prevent cancer. Additionally, aMEDcontaining models performed equally well or better than models containing WCRF/AICR scores in our study population.

In conclusion, high adherence to the MD was not associated with decreased risks of advanced prostate cancer as well as total, noninvasive and invasive bladder cancer in the NLCS. The positive associations that we observed between MD adherence and nonadvanced prostate cancer risk potentially resulted from higher levels of care-seeking behavior, screening attendance and prostate cancer awareness in higher educated men with healthier lifestyles, causing a larger number of nonadvanced prostate cancer diagnoses in this group. Therefore, we recommend that future studies on dietary factors and prostate cancer risk also report results specifically for advanced prostate cancer. 


\section{References}

1. Bray F, Ferlay J, Soerjomataram I, Siegel RL, Torre LA, Jemal A. Global cancer statistics 2018: GLOBOCAN estimates of incidence and mortality worldwide for 36 cancers in 185 countries. CA Cancer J Clin. 2018;68(6):394-424.

2. Hsing AW, Chokkalingam AP. Prostate cancer epidemiology. Front Biosci. 2006;11:1388-413.

3. Pernar CH, Ebot EM, Wilson KM, Mucci LA. The Epidemiology of Prostate Cancer. Cold Spring Harb Perspect Med. 2018;8(12).

4. Cumberbatch MGK, Jubber I, Black PC, Esperto F, Figueroa JD, Kamat AM, et al. Epidemiology of Bladder Cancer: A Systematic Review and Contemporary Update of Risk Factors in 2018. Eur Urol. 2018;74(6):784-95.

5. World Cancer Research Fund / American Institute for Cancer Research. Continuous update project expert report 2018. Diet, nutrition, physical activity and bladder cancer. 2018. Available from: https://www.wcrf.org/dietandcancer.

6. Crawford ED. Understanding the epidemiology, natural history, and key pathways involved in prostate cancer. Urology. 2009;73(5 Suppl):S4-10.

7. Clark PE, Agarwal N, Biagioli MC, Eisenberger MA, Greenberg RE, Herr HW, et al. Bladder cancer. J Natl Compr Canc Netw. 2013;11(4):446-75.

8. Al-Zalabani AH, Stewart KF, Wesselius A, Schols AM, Zeegers MP. Modifiable risk factors for the prevention of bladder cancer: a systematic review of meta-analyses. Eur J Epidemiol. 2016;31(9):811-51.

9. Willett WC, Sacks F, Trichopoulou A, Drescher G, Ferro-Luzzi A, Helsing E, et al. Mediterranean diet pyramid: a cultural model for healthy eating. Am J Clin Nutr. 1995;61(6 Suppl):1402S-6S.

10. Trichopoulou A, Lagiou P. Healthy traditional Mediterranean diet: an expression of culture, history, and lifestyle. Nutr Rev. 1997;55(11 Pt 1):383-9.

11. Fung TT, Rexrode KM, Mantzoros CS, Manson JE, Willett WC, Hu FB. Mediterranean diet and incidence of and mortality from coronary heart disease and stroke in women. Circulation. 2009;119(8):1093-100.

12. Giovannucci E, Liu Y, Platz EA, Stampfer MJ, Willett WC. Risk factors for prostate cancer incidence and progression in the health professionals follow-up study. Int J Cancer. 2007;121(7):15718.

13. Bosire C, Stampfer MJ, Subar AF, Park Y, Kirkpatrick SI, Chiuve SE, et al. Index-based dietary patterns and the risk of prostate cancer in the NIH-AARP diet and health study. Am J Epidemiol. 2013;177(6):504-13.

14. Kenfield SA, DuPre N, Richman EL, Stampfer MJ, Chan JM, Giovannucci EL. Mediterranean diet and prostate cancer risk and mortality in the Health Professionals Follow-up Study. Eur Urol. 2014;65(5):887-94.

15. Buckland G, Ros MM, Roswall N, Bueno-de-Mesquita HB, Travier N, Tjonneland A, et al. Adherence to the Mediterranean diet and risk of bladder cancer in the EPIC cohort study. Int J Cancer. 2014;134(10):2504-11.

16. Dugue PA, Hodge AM, Brinkman MT, Bassett JK, Shivappa N, Hebert JR, et al. Association between selected dietary scores and the risk of urothelial cell carcinoma: A prospective cohort study. Int J Cancer. 2016;139(6):1251-60.

17. van den Brandt PA, Goldbohm RA, van ' $t$ Veer P, Volovics A, Hermus RJ, Sturmans F. A largescale prospective cohort study on diet and cancer in The Netherlands. J Clin Epidemiol. 1990;43(3):28595.

18. van den Brandt PA, Schouten LJ, Goldbohm RA, Dorant E, Hunen PM. Development of a record linkage protocol for use in the Dutch Cancer Registry for Epidemiological Research. Int J Epidemiol. 1990;19(3):553-8.

19. Goldbohm RA, van den Brandt PA, Brants HA, van 't Veer P, Al M, Sturmans F, et al. Validation of a dietary questionnaire used in a large-scale prospective cohort study on diet and cancer. Eur J Clin Nutr. 1994;48(4):253-65.

20. Volovics A, van den Brandt PA. Methods for the analyses of case-cohort studies. Biometrical J. 1997;39(2):195-214.

21. Prentice RL. A case-cohort design for epidemiologic cohort studies and disease prevention 
trials. Biometrika. 1986;73(1):1-11.

22. Goldbohm RA, van ' $t$ Veer P, van den Brandt PA, van ' $t$ Hof MA, Brants HA, Sturmans F, et al. Reproducibility of a food frequency questionnaire and stability of dietary habits determined from five annually repeated measurements. Eur J Clin Nutr. 1995;49(6):420-9.

23. NEVO table. Dutch food composition table 1986-1987. The Hague, the Netherlands: Voorlichtingsbureau voor de Voeding; 1986.

24. Fung TT, McCullough ML, Newby PK, Manson JE, Meigs JB, Rifai N, et al. Diet-quality scores and plasma concentrations of markers of inflammation and endothelial dysfunction. Am J Clin Nutr. 2005;82(1):163-73.

25. Trichopoulou A, Orfanos P, Norat T, Bueno-de-Mesquita B, Ocke MC, Peeters PH, et al. Modified Mediterranean diet and survival: EPIC-elderly prospective cohort study. BMJ. 2005;330(7498):991.

26. Mitrou PN, Kipnis V, Thiebaut AC, Reedy J, Subar AF, Wirfalt E, et al. Mediterranean dietary pattern and prediction of all-cause mortality in a US population: results from the NIH-AARP Diet and Health Study. Arch Intern Med. 2007;167(22):2461-8.

27. Trichopoulou A, Kouris-Blazos A, Wahlqvist ML, Gnardellis C, Lagiou P, Polychronopoulos E, et al. Diet and overall survival in elderly people. BMJ. 1995;311(7018):1457-60.

28. Trichopoulou A, Costacou T, Bamia C, Trichopoulos D. Adherence to a Mediterranean diet and survival in a Greek population. N Engl J Med. 2003;348(26):2599-608.

29. World Cancer Research Fund / American Institute for Cancer Research. Diet, nutrition, physical activity and cancer: a global perspective. Continuous update project expert report 2018. 2018. Available from: https://www.wcrf.org/dietandcancer.

30. Lin DY, Wei LJ. The Robust Inference for the Cox Proportional Hazards Model. J Am Stat Assoc. 1989;84(408):1074-8.

31. Grambsch PM, Therneau TM. Proportional Hazards Tests and Diagnostics Based on Weighted Residuals. Biometrika. 1994;81(3):515-26.

32. de Vogel S, Bongaerts BW, Wouters KA, Kester AD, Schouten LJ, de Goeij AF, et al. Associations of dietary methyl donor intake with MLH1 promoter hypermethylation and related molecular phenotypes in sporadic colorectal cancer. Carcinogenesis. 2008;29(9):1765-73.

33. Wacholder S, Gail MH, Pee D, Brookmeyer R. Alternative Variance and Efficiency Calculations for the Case-Cohort Design. Biometrika. 1989;76(1):117-23.

34. Akaike H. A new look at the statistical model identification. IEEE Transactions on automatic control. 1974;AC-19(6):716-23.

35. van den Brandt PA, Schulpen M. Mediterranean diet adherence and risk of postmenopausal breast cancer: results of a cohort study and meta-analysis. Int J Cancer. 2017;140(10):2220-31.

36. Schulpen $\mathrm{M}$, van den Brandt PA. Adherence to the Mediterranean diet and risk of lung cancer in the Netherlands Cohort Study. Br J Nutr. 2018;119(6):674-84.

37. Schulpen $M$, Peeters PH, van den Brandt PA. Mediterranean diet adherence and risk of esophageal and gastric cancer subtypes in the Netherlands Cohort Study. Gastric Cancer. 2019;22(4):663-74.

38. World Cancer Research Fund / American Institute for Cancer Research. Food, nutrition, physical activity, and the prevention of cancer: a global perspective. Washington DC: American Institute for Cancer Research; 2007.

39. Romaguera D, Vergnaud AC, Peeters PH, van Gils CH, Chan DS, Ferrari P, et al. Is concordance with World Cancer Research Fund/American Institute for Cancer Research guidelines for cancer prevention related to subsequent risk of cancer? Results from the EPIC study. Am J Clin Nutr. 2012;96(1):150-63.

40. Vergnaud AC, Romaguera D, Peeters PH, van Gils CH, Chan DS, Romieu I, et al. Adherence to the World Cancer Research Fund/American Institute for Cancer Research guidelines and risk of death in Europe: results from the European Prospective Investigation into Nutrition and Cancer cohort study. Am J Clin Nutr. 2013;97(5):1107-20.

41. Ax E, Garmo H, Grundmark B, Bill-Axelson A, Holmberg L, Becker W, et al. Dietary patterns and prostate cancer risk: report from the population based ULSAM cohort study of Swedish men. Nutr Cancer. 2014;66(1):77-87.

42. Lavalette C, Adjibade M, Srour B, Sellem L, Fiolet T, Hercberg S, et al. Cancer-Specific and General Nutritional Scores and Cancer Risk: Results from the Prospective NutriNet-Sante Cohort. 
Cancer Res. 2018;78(15):4427-35.

43. Moller E, Galeone C, Andersson TM, Bellocco R, Adami HO, Andren O, et al. Mediterranean Diet Score and prostate cancer risk in a Swedish population-based case-control study. J Nutr Sci. 2013;2:e15.

44. Russo GI, Solinas T, Urzi D, Privitera S, Campisi D, Cocci A, et al. Adherence to Mediterranean diet and prostate cancer risk in Sicily: population-based case-control study. Int J Impot Res. 2019;31(4):269-75.

45. Jahn JL, Giovannucci EL, Stampfer MJ. The high prevalence of undiagnosed prostate cancer at autopsy: implications for epidemiology and treatment of prostate cancer in the Prostate-specific Antigen-era. Int J Cancer. 2015;137(12):2795-802.

46. Witlox WJA, van Osch FHM, Brinkman M, Jochems S, Goossens ME, Weiderpass E, et al. An inverse association between the Mediterranean diet and bladder cancer risk: a pooled analysis of 13 cohort studies. Eur J Nutr. 2019.

47. Bravi F, Spei ME, Polesel J, Di Maso M, Montella M, Ferraroni M, et al. Mediterranean Diet and Bladder Cancer Risk in Italy. Nutrients. 2018;10(8).

48. Mizoue T, Kasai H, Kubo T, Tokunaga S. Leanness, smoking, and enhanced oxidative DNA damage. Cancer Epidemiol Biomarkers Prev. 2006;15(3):582-5.

49. Brill JB. The Mediterranean diet and your health. Am J Lifestyle Med. 2009;3(1):44-56.

50. Verberne L, Bach-Faig A, Buckland G, Serra-Majem L. Association between the Mediterranean diet and cancer risk: a review of observational studies. Nutr Cancer. 2010;62(7):860-70. 
Chapter 7

Supplementary materials

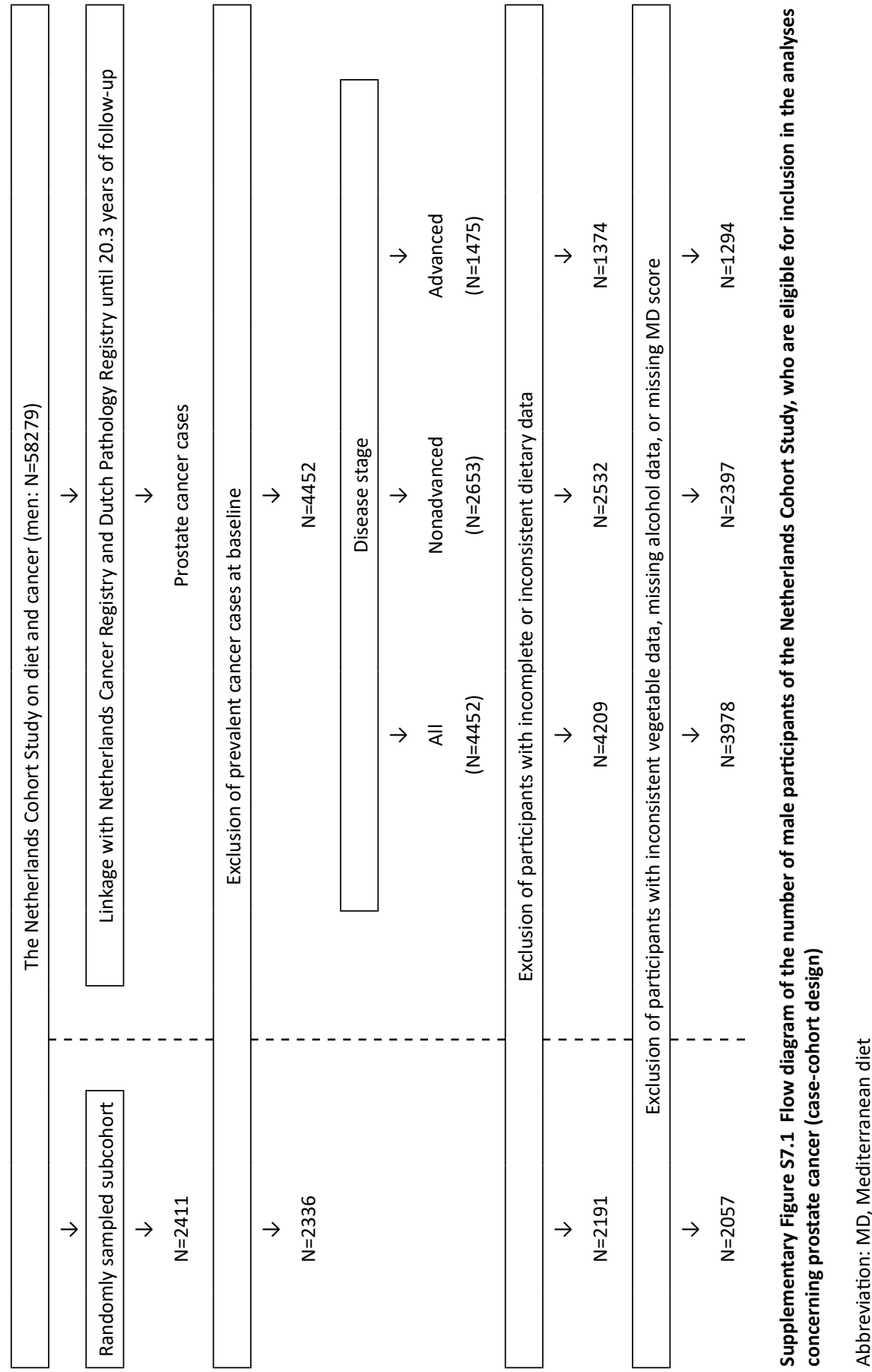




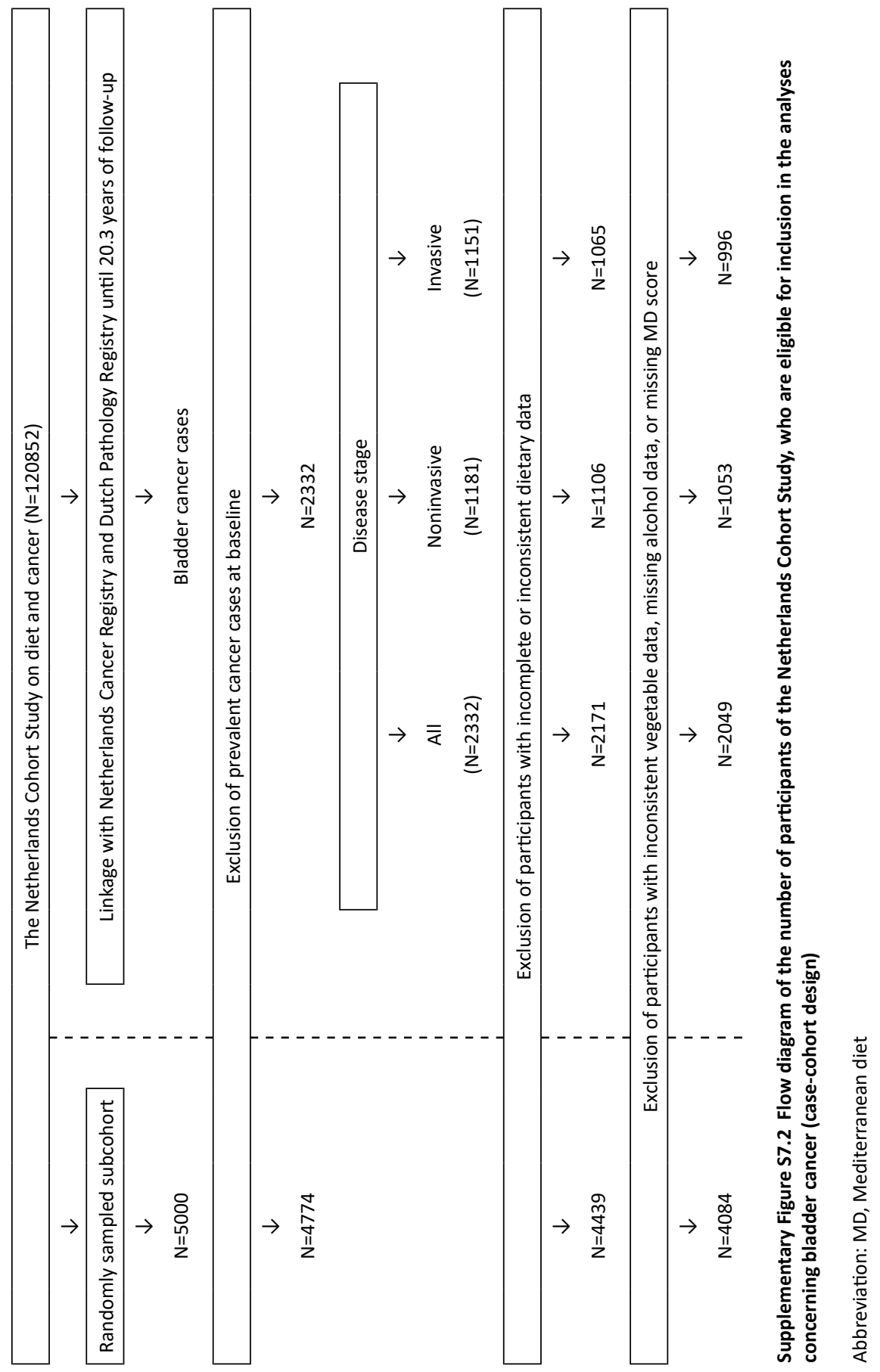




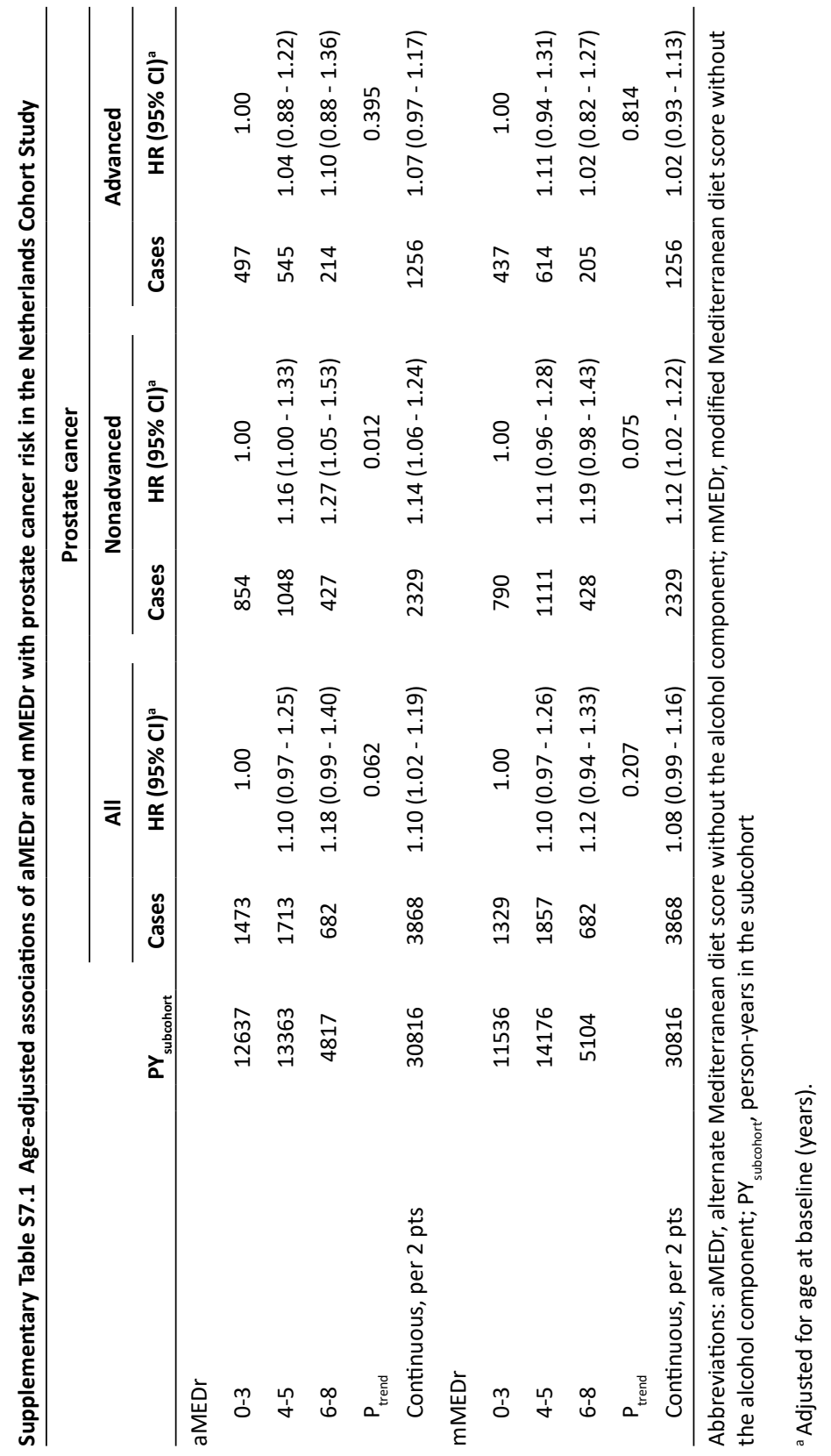




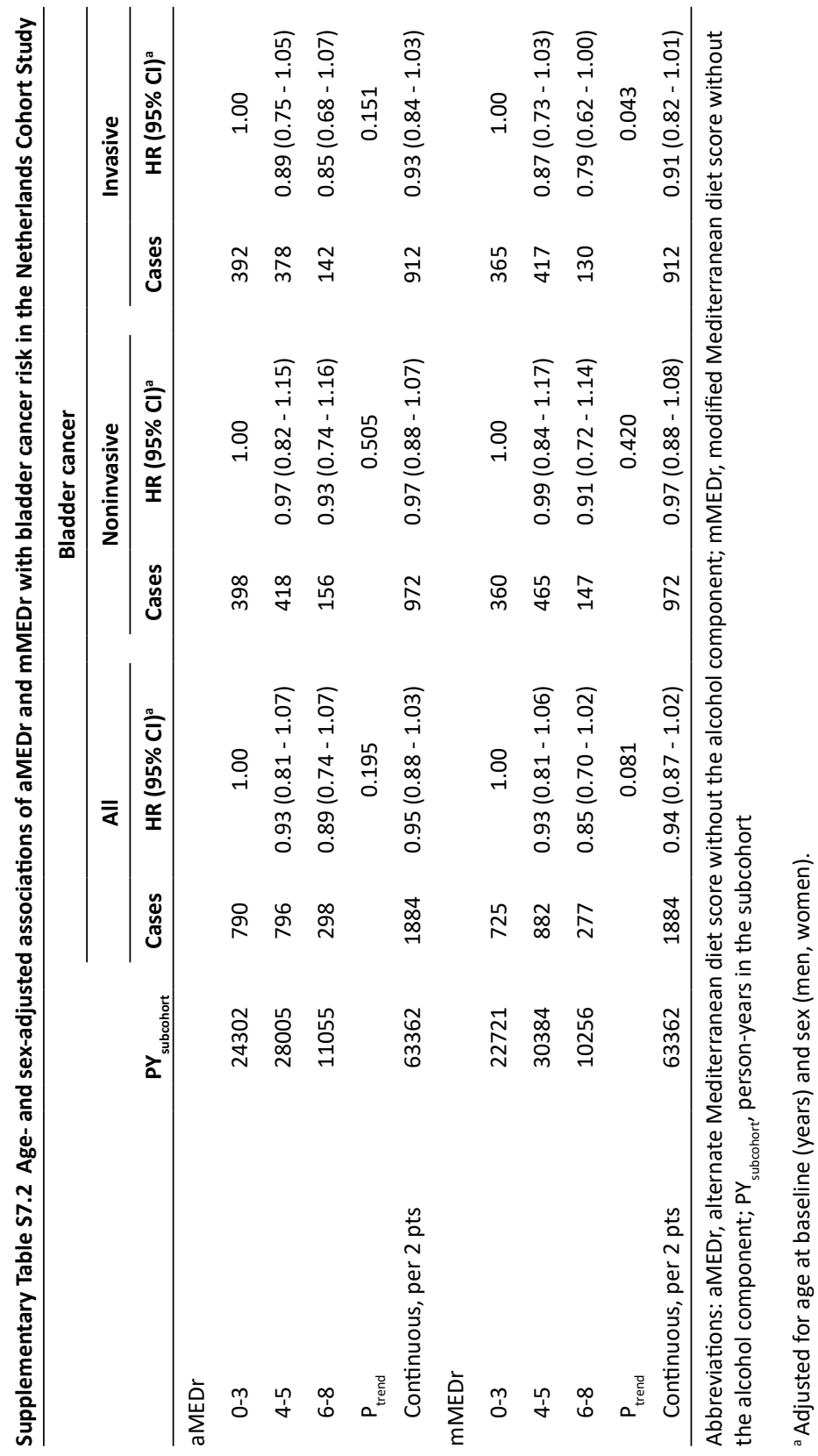




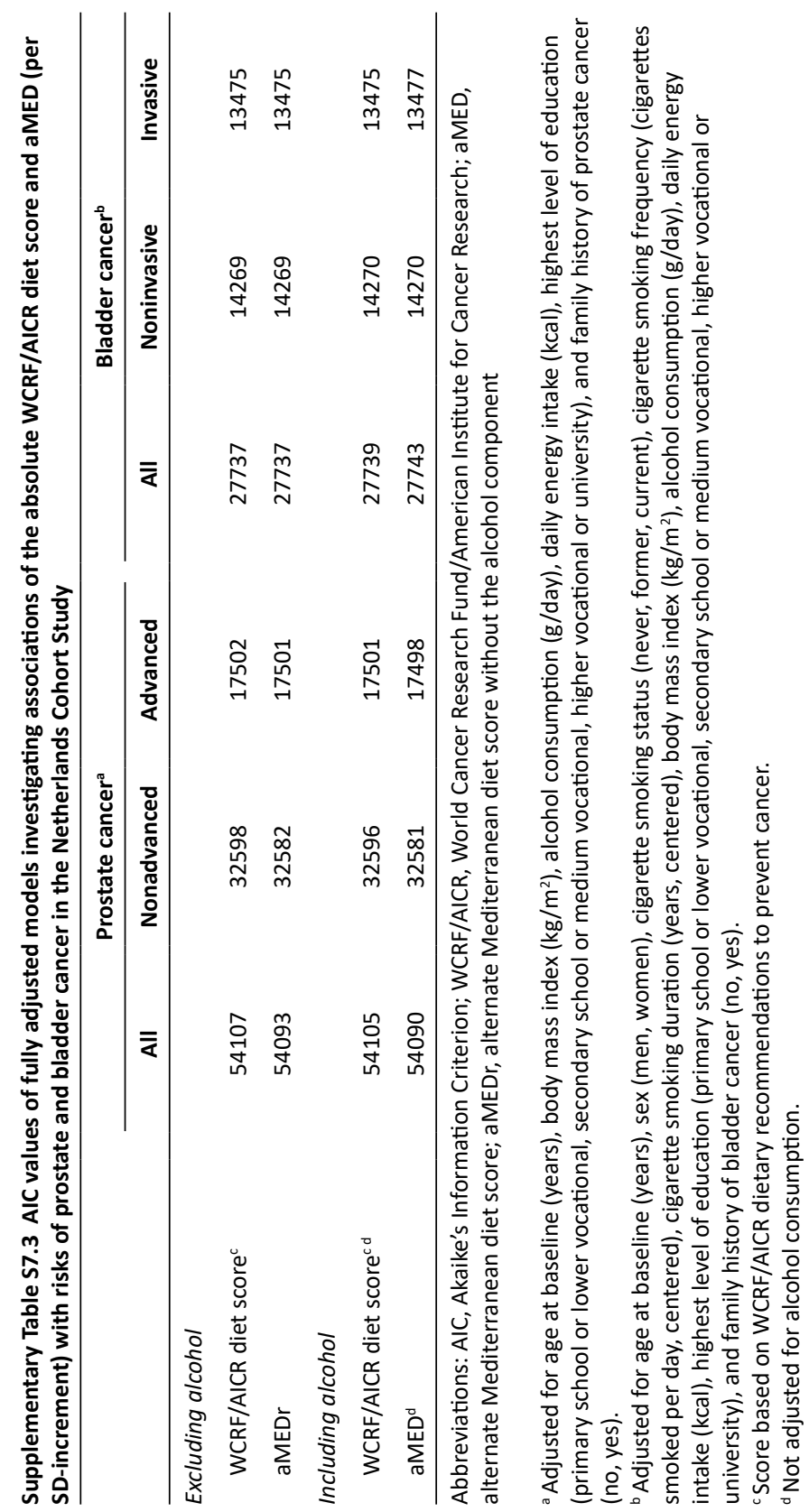


Mediterranean diet and risk of prostate and bladder cancer 


\section{Chapter 8}

\section{Adherence to the Mediterranean diet and overall cancer incidence: the Netherlands Cohort Study}
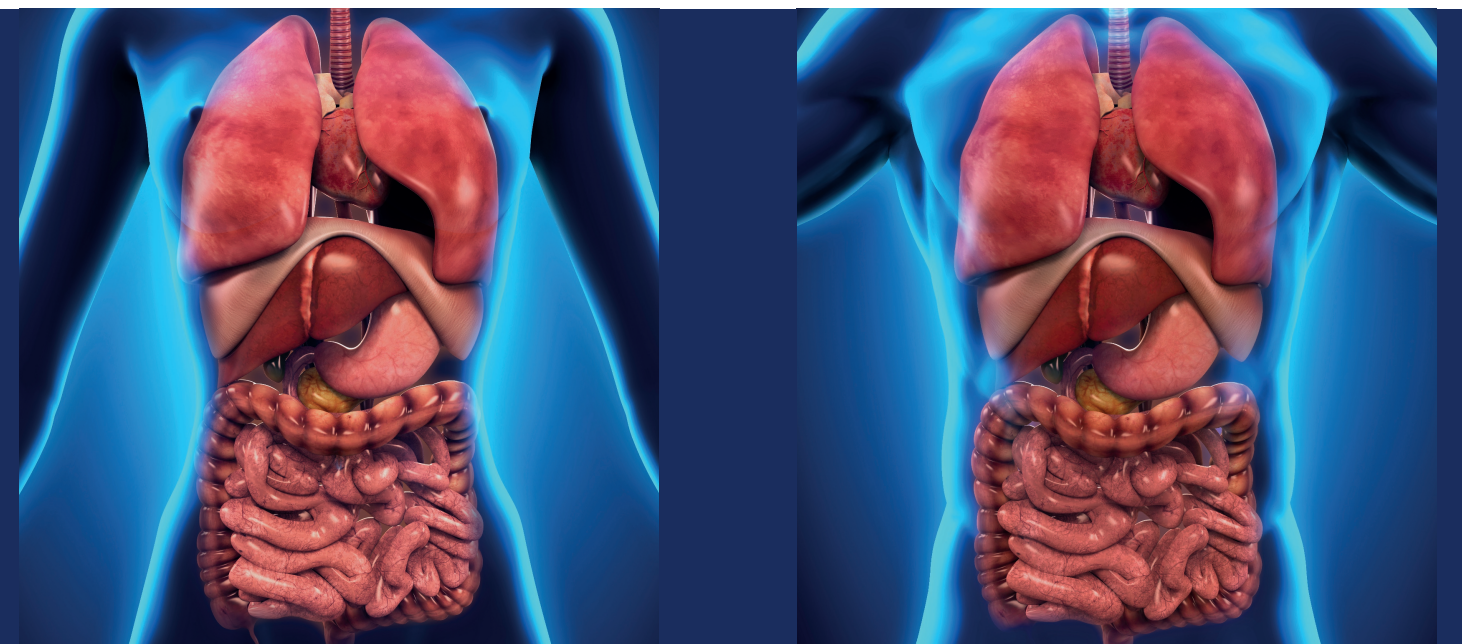

Maya Schulpen, Piet A. van den Brandt 


\begin{abstract}
Background

Adherence to the plant-based Mediterranean diet (MD) has been associated with reduced risks of various cancer types. However, prospective findings for overall cancer risk are inconclusive. In this analysis of the Netherlands Cohort Study (NLCS), we examined sexspecific relations of MD adherence with overall cancer risk.
\end{abstract}

\title{
Methods
}

Baseline data on diet and other cancer risk factors were available for 120852 NLCS participants, who had a maximum follow-up of 20.3 years. The alternate Mediterranean diet score without alcohol (aMEDr) was our principal measure of MD adherence. The Cox regression analyses (case-cohort design) included 12184 male and 7071 female cancer cases, respectively, who had complete data on potential confounders.

\section{Results}

Higher MD adherence (aMEDr) was associated with a non-significantly reduced overall cancer risk in women in continuous analyses $\left[\mathrm{HR}_{\text {per 2-point increment }}(95 \% \mathrm{Cl})\right.$ : $\left.0.93(0.86-1.01)\right]$. Categorical analyses in women pointed in the same direction. The inverse association was statistically significant when comparing middle (4-5) to low (0-3) aMEDr values with a HR of 0.85. In contrast, no association was observed in men $\left[\mathrm{HR}_{\text {per 2-point increment }}(95 \% \mathrm{Cl}): 1.02(0.95\right.$ - 1.10)]. Results for cancer subgroups, defined by relations with tobacco smoking, obesity, and alcohol consumption, were largely similar to the overall findings. Model fits diminished when alcohol was included in the MD score.

\section{Conclusions}

In the NLCS, higher MD adherence seemed to be associated with a reduced overall cancer risk in women, but not in men. However, the inverse associations in women were generally not statistically significant in multivariable-adjusted analyses. 


\section{Introduction}

Cancer poses a large social and economic burden to the world's population with an estimated 18.1 million incident cases and 9.6 million deaths in 2018 [1]. Additionally, cancer was the leading cause of premature mortality in Northern America and the majority of Europe in 2015 [1]. The cancer incidence and mortality are increasing rapidly as a result of the growing and aging world population along with changes in the prevalence and distribution of the major cancer risk factors [1]. Lanting et al. estimated that just under $10 \%$ of the cancer diagnoses in the Netherlands in 2010 could be attributed to a less than optimal diet [2].

The traditional Mediterranean diet (MD) of the early 1960s is characterized by a high consumption of plant foods (i.e., vegetables, legumes, fruits, nuts, and whole grains). Meat and dairy products are consumed in low to moderate amounts, which in combination with the abundant use of olive oil leads to the high ratio of monounsaturated to saturated fatty acids (MUFA:SFA ratio) that is characteristic of the MD. Alcohol, particularly wine, is consumed in moderate amounts in the traditional MD $[3,4]$.

MD adherence has been associated with reduced risks of multiple, but not all, types of cancer [5-8]. To gain insight into the overall benefits of MD adherence in view of cancer prevention and to facilitate the formulation of a clear public health message to the general public or policymakers, it is useful to get an impression of the association of MD adherence with overall cancer risk. The currently available prospective evidence for the potential relation between a priori defined MD adherence and overall cancer risk is inconclusive [913]. Higher MD adherence was associated with a reduced overall cancer risk in some studies $[9,10,13]$, but not in others $[11,12]$. Furthermore, the potential inverse relation might be stronger in women compared to men [9].

The aim of the present study was to evaluate the association between a priori defined $\mathrm{MD}$ adherence and overall cancer risk in men and women participating in the prospective Netherlands Cohort Study (NLCS). In addition to overall cancer risk, we investigated associations of MD adherence with risks of cancer subgroups defined by relations with tobacco smoking, obesity, and alcohol consumption. Finally, performances of models including MD scores with and without alcohol were compared.

\section{Materials and methods}

\section{Study population and cancer follow-up}

The prospective NLCS was initiated in September 1986 [14-17]. In total, 58279 men and 62573 women, aged 55 to 69 years, completed the baseline questionnaire on cancer risk factors, including diet. A case-cohort approach was used to process and analyze the data $[14,17,18]$. Therefore, a subcohort $(\mathrm{N}=5000)$ was randomly selected immediately after baseline and biennially followed up for vital status to estimate accumulated person-time at risk in the whole cohort. Subcohort members contributed to the number of personyears at risk from baseline until December 31, 2006 or censoring (cancer diagnosis, death, emigration, or loss to follow-up). The NLCS was approved by institutional review boards from Maastricht University and the Netherlands Organization for Applied Scientific Research. Study participants consented to participation by filling out the baseline questionnaire.

During 20.3 years of follow-up, 25848 cases diagnosed with a microscopically confirmed, 
first primary cancer (excluding basal cell carcinoma of the skin) were detected in the total NLCS cohort through annual record linkage with the Netherlands Cancer Registry and the nationwide Dutch Pathology Registry (PALGA) [15]. Study participants reporting prevalent cancer at baseline (except skin cancer) were excluded. Additional exclusion of subjects with incomplete or inconsistent data regarding diet, alcohol, or MD adherence left 22228 cancer cases (men: 13657, women: 8571) and 4084 subcohort members (men: 2057, women: 2027), who were eligible for inclusion in the present analysis (Figure 8.1). In addition to overall cancer incidence, incidence of cancers known to be related to tobacco smoking, obesity, and alcohol consumption were considered as secondary end points. The subgroup of smoking-related cancers comprised cancers of the oral cavity (including lip) and pharynx, esophagus, stomach, colorectum, liver, pancreas, nasal cavity and paranasal sinuses, larynx, trachea, lung, uterine cervix, ovary, kidney, ureter, and urinary bladder as well as myeloid leukemia $[10,13,19,20]$. Obesity-related cancers were defined as cancers of the esophagus (adenocarcinoma), stomach (cardia), colorectum, liver, gallbladder, pancreas, breast, corpus uteri, ovary, kidney, and thyroid, and multiple myeloma [13, 20, 21]. Finally, alcoholrelated cancers included cancers of the oral cavity (including lip) and pharynx, esophagus (squamous cell carcinoma), colorectum, liver, larynx, and breast $[10,19,20]$. Results for the cancer subgroups were compared to results obtained combining all other cancers (i.e., cancers not classified as being related to tobacco smoking, obesity, or alcohol consumption, respectively).

\section{Exposure assessment}

The NLCS baseline questionnaire included a 150 -item, semi-quantitative food frequency questionnaire (FFQ) focusing on the study participant's dietary habits over the past 12 months. This FFQ performed adequately as judged by comparison with nine-day diet records [16] and intakes of most nutrients were found to be relatively stable for over at least five years [22]. Mean daily nutrient intakes were calculated from the FFQ data utilizing the Dutch food composition table of the year 1986 [23].

\section{Mediterranean diet adherence}

The relative level of MD adherence was determined using the alternate Mediterranean diet score (aMED) $[24,25]$, which is a variation of the original traditional Mediterranean diet score (tMED) [26, 27]. aMED is composed of nine dietary components (scored by 0 or 1 points each), which are typical of the MD $[24,25]$. Subjects obtain 1 point for mean daily intakes at or above the sex-specific median of vegetables (excluding potatoes), legumes, fruits, nuts, whole grains, and fish. Opposite scoring is applied to the intake of red and processed meats. Finally, scores of 1 point are assigned to a moderate alcohol consumption of 5 to 25 grams per day and a high ( $\geq$ sex-specific median) MUFA:SFA ratio. Thus, a maximum score of 9 points can be obtained, reflecting the highest level of $M D$ adherence $[24,25]$. Food intakes were adjusted to daily energy intakes of 2500 (men) and 2000 (women) kilocalories to control for differences in energy intake $[25,26]$.

Furthermore, we created a reduced variant from the original aMED that did not contain the alcohol component (aMEDr) $[6,28]$, because alcohol consumption has been associated with an increased risk of multiple cancer types even at moderate levels [19, 29, 30]. Therefore, we consider aMEDr our primary measure of MD adherence. Based on their MD score value, subjects were classified as having low $(\leq 3)$, middle (4-5), or high $(\geq 6)$ levels of MD adherence 


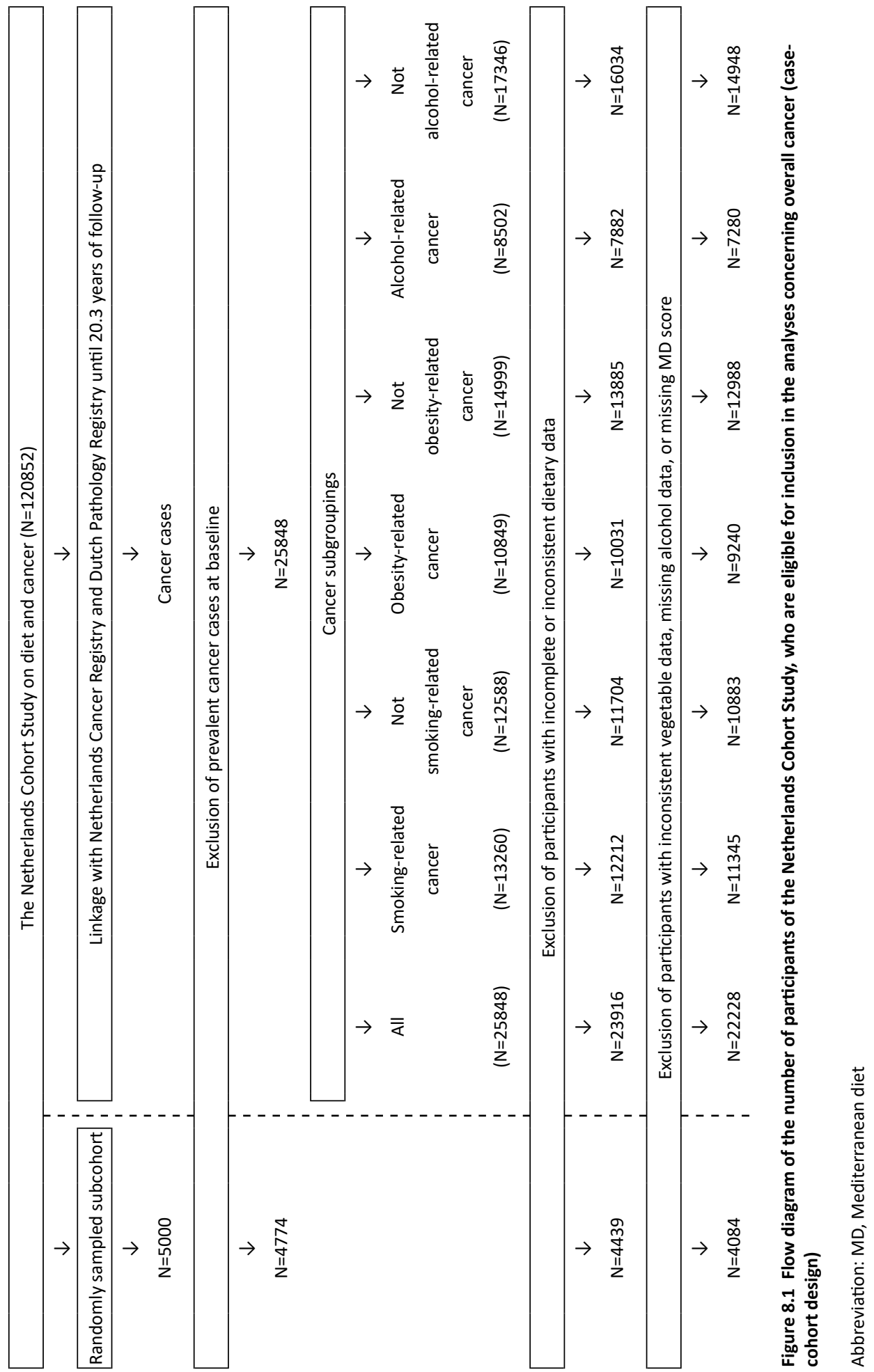


[25]. Additionally, the MD scores were continuously modelled per 2-point increase.

\section{Statistical analyses}

Sex-specific hazard ratios (HRs) and 95\% confidence intervals (95\% Cls) for the relation between MD adherence and overall cancer incidence were estimated by Cox proportional hazards modelling using duration of follow-up as time variable. Standard errors of the HRs were estimated using the robust Huber-White sandwich estimator, which accounts for the additional variance associated with sampling from the total cohort [31]. The validity of the proportional hazards $(\mathrm{PH})$ assumption was evaluated by scaled Schoenfeld residuals tests [32]. Because of the large number of cases, these tests may easily yield significant results. Therefore, we also visually inspected $-\ln (-\operatorname{In})$ survival plots and concluded that the $\mathrm{PH}$ assumption was met for the exposure variables.

Age- and multivariable-adjusted effect estimates were obtained for the MD scores, which were modelled as categorical and continuous terms. The multivariable-adjusted HRs were corrected for potential confounding by age at baseline, cigarette smoking (status, frequency, and duration), body mass index (BMI), height, alcohol consumption (except for the original aMED including alcohol), total daily energy intake, highest level of education, non-occupational physical activity, and family history of cancer. Effect estimates obtained among women were additionally adjusted for reproductive factors (age at menarche, parity, age at first birth, age at menopause, oral contraceptive use, and use of postmenopausal hormone replacement therapy). All potential confounders were predefined and selected from the literature. For each adherence category, sex-specific median MD score values were determined in the subcohort. By fitting these values as continuous terms in the Cox regression models, we tested for linear trends. Akaike's Information Criterion (AIC) was used to evaluate whether inclusion of alcohol in the MD score affected the model performance [33]. Besides overall cancer incidence, we also estimated sex-specific associations of aMEDr with incidence of smoking-, obesity-, and alcohol-related cancers as well as cancers not classified as being related to these factors. Statistical significance of differences in HRs obtained for cancers related vs. not related to tobacco smoking, obesity, or alcohol consumption was assessed using a competing risks procedure [34]. Standard errors for the observed differences in HRs were estimated using a bootstrapping method developed for the case-cohort design [35].

Furthermore, sex-specific associations between aMEDr and overall cancer risk were estimated within strata of cigarette smoking status, alcohol consumption, BMI, educational level, and family history of cancer. To assess the statistical significance of potential differences across strata, Wald tests were performed on interaction terms between aMEDr and the stratifying covariates. Finally, our main analyses were repeated excluding the first two years of follow-up to check for potential reversed causation, since the presence of preclinical cancer at baseline could have influenced dietary habits. Analyses were performed using Stata software (version 15). Statistical significance was indicated by a two-sided $p$-value $<0.05$. 


\section{Results}

Table 8.1 summarizes baseline characteristics of male and female subcohort members and cancer cases. The mean (SD) values of aMEDr were 3.9 (1.6) and 4.0 (1.6) in male and female subcohort members, respectively. Largely comparable aMEDr values were observed in cancer cases. Cancer cases of both sexes were more likely to smoke, had a higher BMI, and more often reported a family history of cancer compared to subcohort members. Furthermore, male cancer cases had a higher level of alcohol consumption and were more physically active. In contrast, a lower level of physical activity was observed in female cancer cases compared to the subcohort. Concerning reproductive factors, female cancer cases were more frequently nulliparous, older at the birth of their first child, and less often users of oral contraceptives than subcohort members.

Age- and multivariable-adjusted $\mathrm{HRs}$ and $95 \% \mathrm{Cls}$ for associations of MD adherence with overall cancer risk are shown in Table 8.2, for men and women separately. Of the eligible study population, 3499 subcohort members (men: 1834, women: 1665) and 19255 cancer cases (men: 12184, women: 7071) had complete data on all potential confounders and could be included in the Cox regression analyses.

MD adherence was not associated with overall cancer risk in men in age- and multivariableadjusted analyses (Table 8.2). Multivariable-adjusted HRs (95\% Cls) for aMEDr were 0.99 $(0.84-1.17)$ comparing the highest to the lowest adherence category and $1.02(0.95-1.10)$ per 2-point increase in score, respectively. Although aMEDr was not significantly associated with any of the cancer subgroups in men (Table 8.3), HRs were statistically significantly different for cancers related vs. not related to tobacco smoking, obesity, and alcohol consumption [ $p_{\text {heterogeneity }}:<0.001$ (smoking), 0.008 (obesity), and 0.047 (alcohol)].

In women, higher aMEDr values were associated with a significantly reduced overall cancer risk in age-adjusted analyses $\left[\mathrm{HR}_{\text {high vs. low }}(95 \% \mathrm{Cl}): 0.84(0.72-0.99), \mathrm{HR}_{\text {per 2-point increment }}(95 \% \mathrm{Cl})\right.$ : $0.91(0.85-0.98)$ ] (Table 8.2). After additional adjustment for other potential confounding factors, the inverse associations slightly attenuated and lost statistical significance in most cases $\left[\mathrm{HR}_{\text {high vs. low }}(95 \% \mathrm{Cl}): 0.90(0.76-1.06), \mathrm{HR}_{\text {per 2-point increment }}(95 \% \mathrm{Cl}): 0.93(0.86-1.01)\right]$. However, a statistically significantly reduced overall cancer risk persisted when comparing the middle to the lowest aMEDr category, with a HR $(95 \% \mathrm{Cl})$ of $0.85(0.75-0.97)$. Multivariableadjusted associations of aMEDr with subgroups of cancers related/not related to tobacco smoking, obesity, and alcohol consumption were comparable to those obtained for overall cancer risk in women (Table 8.3). Furthermore, heterogeneity tests confirmed similarity of associations for cancers related vs. not related to these lifestyle factors [ $p_{\text {heterogeneity }}: 0.442$ (smoking), 0.188 (obesity), and 0.309 (alcohol)].

For both sexes, largely comparable HRs and 95\% Cls for overall cancer risk were obtained when alcohol was included in the MD score. However, AIC values were higher for models in which MD adherence was assessed using the MD score variant including alcohol (aMED), indicating a worse fit. Associations between aMEDr and overall cancer risk within strata of potential effect modifying factors are presented in Table 8.4. The relation of aMEDr with overall cancer risk in men became more positive with increasing level of education $\left(p_{\text {interaction }}=0.049\right)$, reaching statistical significance in the highest category. Although aMEDr did not significantly interact with educational level in women, a similar pattern was observed. Associations did not significantly differ across strata of cigarette smoking status, alcohol consumption, BMI, and family history of cancer in both men and women. Excluding the first two years of follow-up did not essentially change the associations. 


\section{Chapter 8}

Table 8.1 Sex-specific baseline characteristics of subcohort members and cancer cases in the Netherlands Cohort Study

\begin{tabular}{|c|c|c|c|c|c|c|c|c|}
\hline \multirow[b]{4}{*}{ aMEDr } & \multicolumn{4}{|c|}{ Men } & \multicolumn{4}{|c|}{ Women } \\
\hline & \multirow{2}{*}{\multicolumn{2}{|c|}{$\begin{array}{l}\text { Subcohort } \\
\mathbf{N}=\mathbf{2 0 5 7}\end{array}$}} & \multirow{2}{*}{\multicolumn{2}{|c|}{$\begin{array}{l}\text { Cancer cases } \\
\mathrm{N}=13657\end{array}$}} & \multirow{2}{*}{\multicolumn{2}{|c|}{$\begin{array}{l}\text { Subcohort } \\
\mathrm{N}=2027\end{array}$}} & \multirow{2}{*}{\multicolumn{2}{|c|}{$\begin{array}{l}\text { Cancer cases } \\
\mathrm{N}=8571\end{array}$}} \\
\hline & & & & & & & & \\
\hline & 3.9 & $(1.6)$ & 3.9 & $(1.6)$ & 4.0 & $(1.6)$ & 3.9 & $(1.6)$ \\
\hline Age (years) $^{a}$ & 61 & (7) & 62 & (7) & 61 & (7) & 61 & (7) \\
\hline Current cigarette smokers (\%) & 35.1 & & 40.3 & & 21.3 & & 23.1 & \\
\hline Higher vocational education or university (\%) & 19.3 & & 19.6 & & 9.5 & & 9.1 & \\
\hline Alcohol consumption (g/day) ${ }^{a}$ & 9.7 & (20.9) & 11.4 & $(22.0)$ & 1.6 & $(7.8)$ & 1.5 & $(8.6)$ \\
\hline Daily energy intake (kcal) & 2162 & $(501)$ & 2165 & $(494)$ & 1687 & $(392)$ & 1682 & $(386)$ \\
\hline Body mass index $\left(\mathrm{kg} / \mathrm{m}^{2}\right)$ & 24.9 & $(2.6)$ & 25.0 & $(2.6)$ & 25.0 & $(3.5)$ & 25.2 & (3.6) \\
\hline Height $(\mathrm{cm})^{\mathrm{a}}$ & 176 & (9) & 176 & (9) & 165 & (8) & 166 & (8) \\
\hline Non-occupational physical activity (min/day) ${ }^{a}$ & 62.1 & $(67.1)$ & 64.3 & $(65.7)$ & 54.3 & $(52.9)$ & 51.4 & $(53.6)$ \\
\hline Family history of cancer (\%) & 45.1 & & 48.2 & & 47.3 & & 51.5 & \\
\hline Age at menarche (years) ${ }^{a}$ & & & & & 13 & (3) & 13 & (3) \\
\hline Age at menopause (years) ${ }^{a}$ & & & & & 50 & (6) & 50 & (6) \\
\hline Nulliparous (\%) & & & & & 18.5 & & 19.1 & \\
\hline Age at first birth $\geq 30$ years ( $\%$ of parous) & & & & & 22.5 & & 23.3 & \\
\hline Ever use of oral contraceptives (\%) & & & & & 25.4 & & 24.5 & \\
\hline Ever use of hormone replacement therapy (\%) & & & & & 13.4 & & 13.3 & \\
\hline
\end{tabular}

Abbreviation: aMEDr, alternate Mediterranean diet score without the alcohol component

The $\%$ missing values in the total eligible population was $<5 \%$ for all variables included in this table, with the exception of age at menopause in women (6.4\%). Mean (SD) values are reported unless otherwise specified. ${ }^{a}$ Median (IQR) values are reported. 


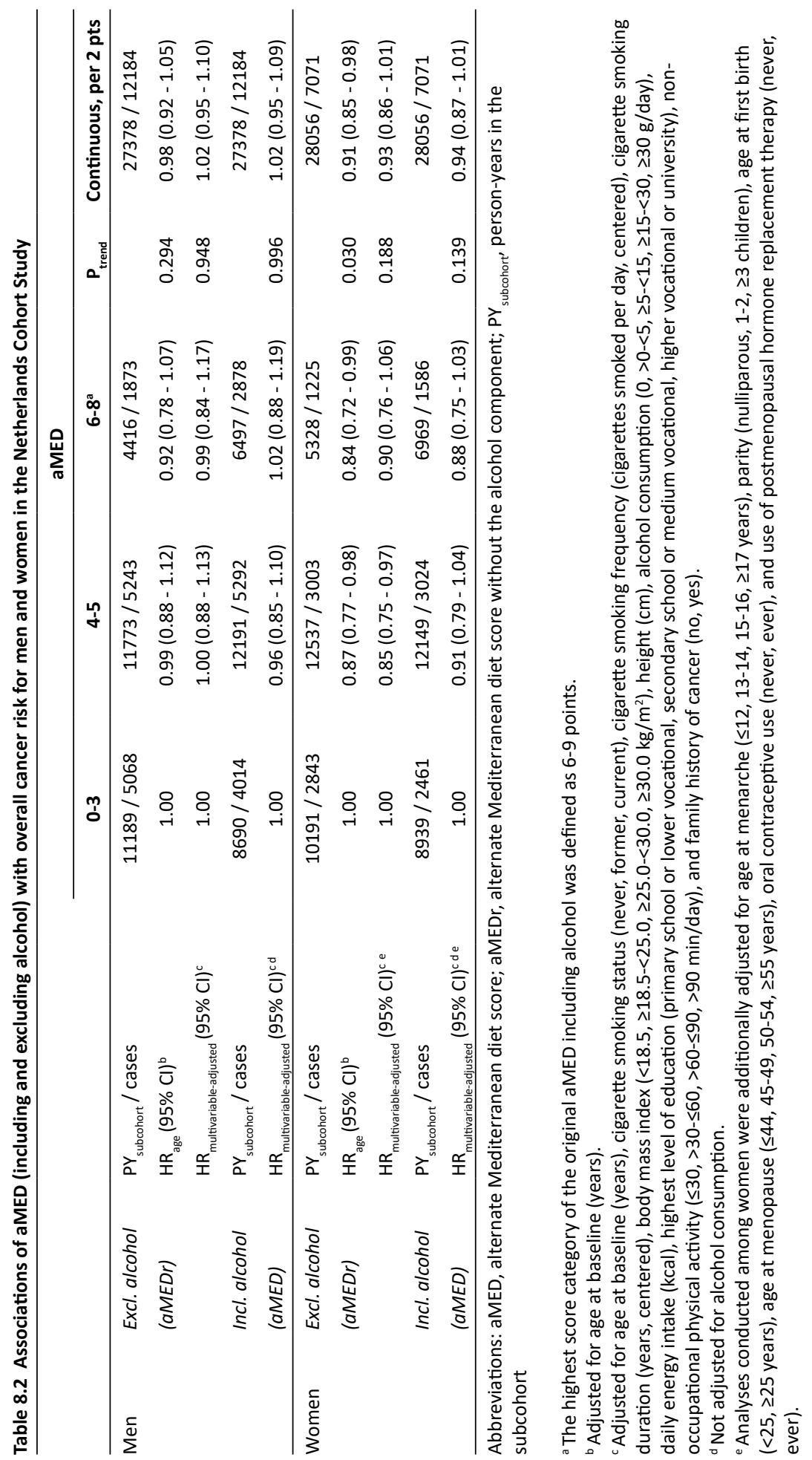




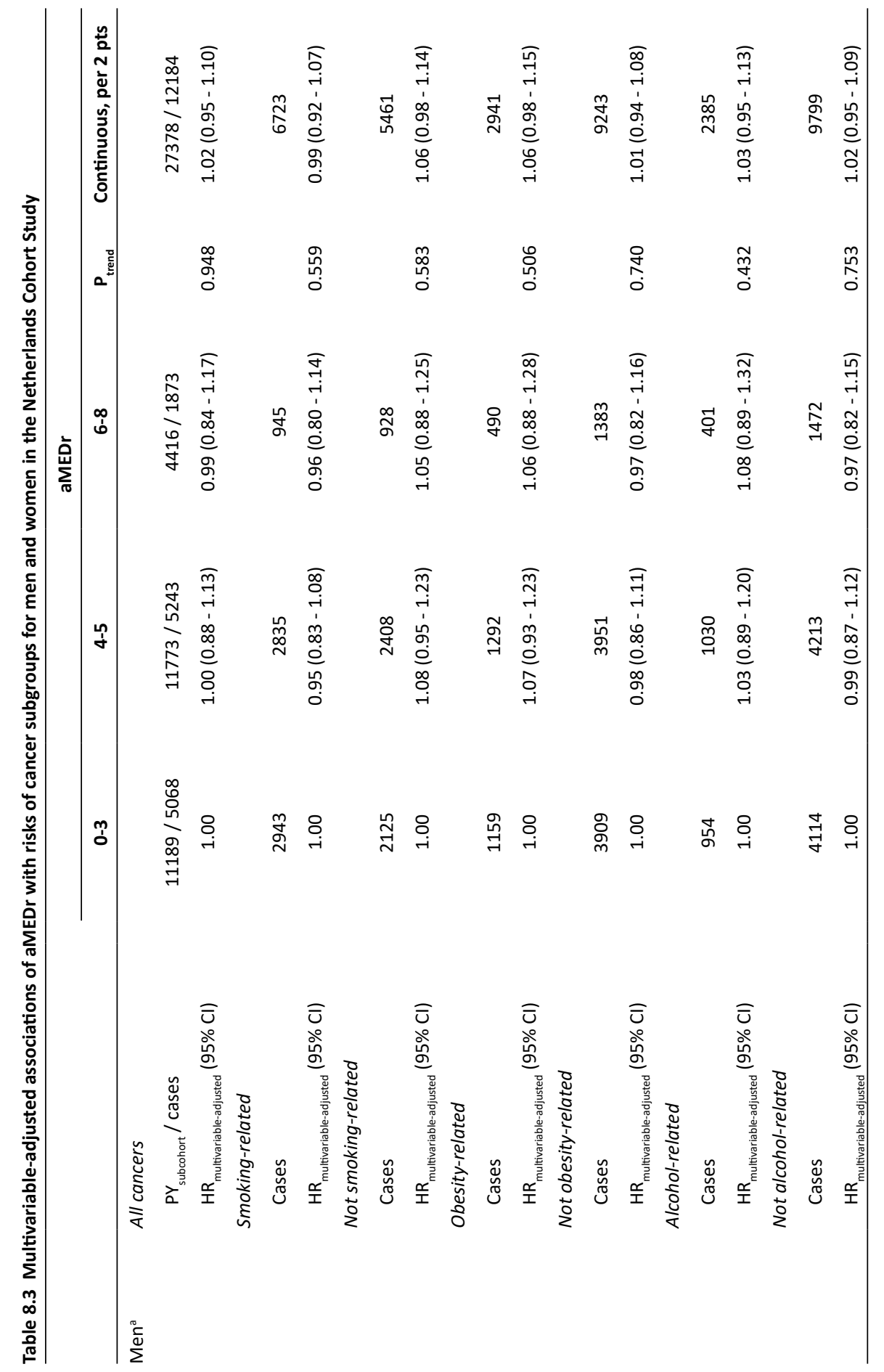




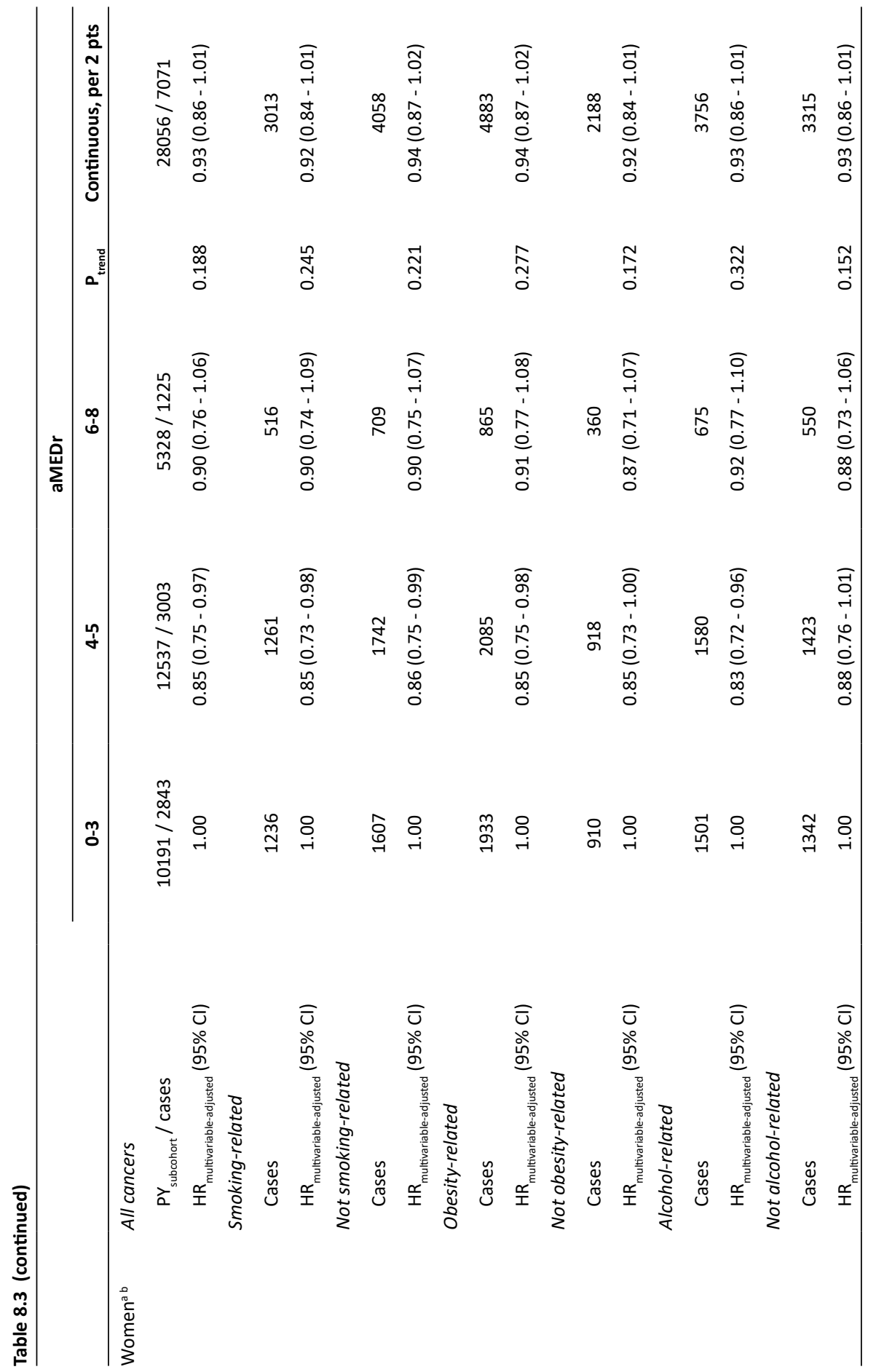




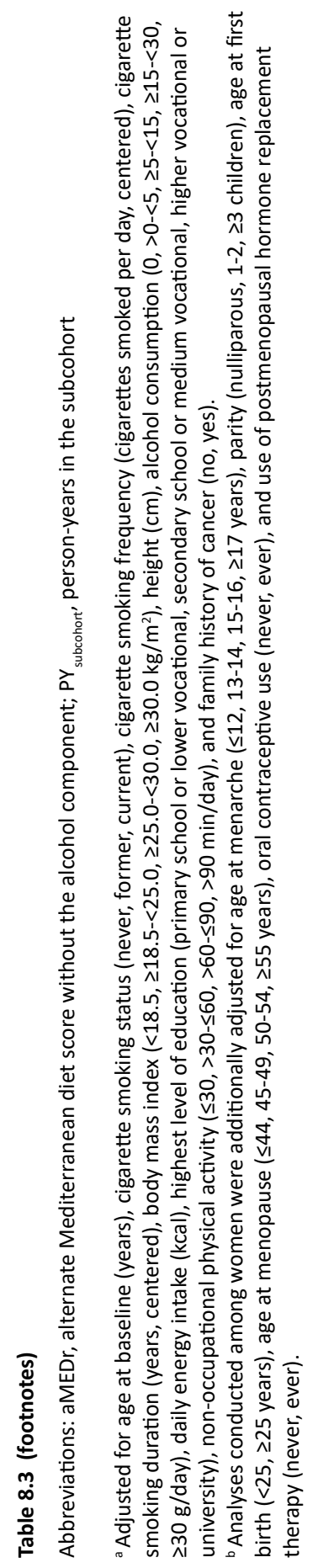




\section{Discussion}

In this NLCS analysis, we have investigated sex-specific associations of a priori defined MD adherence with risks of overall cancer and cancer subgroups defined by relations with three major cancer risk factors (tobacco smoking, obesity, and alcohol consumption). No association was observed between aMEDr and risk of overall cancer or any of the cancer subgroups in men. In contrast, higher aMEDr values seemed to be associated with a reduced risk of cancer (subgroups) in women. However, the inverse associations in women were generally not statistically significant in multivariable-adjusted analyses. Inclusion of alcohol in the MD score diminished the model performance.

A priori defined MD adherence previously has been associated with a significantly reduced overall cancer risk in the total European Prospective Investigation into Cancer and Nutrition (EPIC) cohort as well as the Greek EPIC cohort $[9,10]$. Comparing the highest to the lowest MD adherence category in the total EPIC cohort, HRs $(95 \% \mathrm{Cls})$ of $0.93(0.88-0.99)$ and $0.93(0.89-0.96)$ were observed for men and women, respectively [10]. Although inverse associations were also suggested for both sexes in the Greek EPIC cohort, only effect estimates obtained in women reached statistical significance $\left[\mathrm{HR}_{\text {high v. low }}(95 \% \mathrm{Cl}): 0.83(0.63\right.$ - 1.09) for men and $0.73(0.56-0.96)$ for women] [9]. In addition to the abovementioned EPIC studies, weak inverse associations between $\mathrm{MD}$ adherence and overall cancer risk were observed in men [HR (95\% Cl): $0.97(0.94-1.01)]$ and women [HR increase $(95 \% \mathrm{Cl}): 0.97(0.93-1.00)$ ] participating in the Swedish prospective Västerbotten Intervention Programme [13]. In our present analysis of the NLCS cohort, a priori defined MD adherence was not associated with overall cancer risk in men. However, an inverse association was suggested in women. Although the multivariable-adjusted associations in female NLCS participants were mostly not statistically significant, effect estimates were stronger inverse than those observed for women in the total EPIC cohort, which did reach statistical significance possibly due to the larger number of cases [10]. Additional cohort studies in Germany and France have investigated the association between MD adherence and overall cancer risk in men and women together, and did not observe an association [11, 12]. Besides the prospective cohort evidence, a reduced overall cancer risk (not significant) was indicated in patients with coronary heart disease who followed an $\alpha$-linolenic acid-rich Mediterranean-type diet as opposed to a control diet close to the step 1 prudent diet of the American Heart Association in the randomized Lyon Diet Heart Study [36]. However, results should be interpreted with caution as they were based on only 24 incident cancer cases.

Differential adjustment for potential confounding factors and residual confounding, particularly by tobacco smoking and female reproductive factors, may have contributed to the varying associations between a priori defined MD adherence and overall cancer risk that have been reported thus far. Other potentially contributing factors include differences in the method of MD assessment, the composition of the study population, and the time period and/or geographical region in which the study was conducted. The distribution of the specific cancer types in the overall cancer outcome is likely to vary over time and between countries because of, e.g., different distributions of risk factors and the introduction of cancer screening programs. Some specific cancer types are inversely associated with MD adherence, whereas null associations have been observed for others. For example, MD adherence was inversely associated (not always significantly) with postmenopausal breast and lung cancer risk in previous NLCS analyses [6, 28]. In contrast, we found no association with colorectal cancer risk and a positive association with nonadvanced prostate cancer risk $[37,38]$. Therefore, differences in the relative incidence of specific cancer types could also 
Table 8.4 Sex-specific and multivariable-adjusted associations of aMEDr (per two-point increment) with overall cancer risk for strata of various risk factors in the Netherlands Cohort Study

\begin{tabular}{|c|c|c|c|c|}
\hline & \multicolumn{4}{|c|}{ Overall cancer } \\
\hline & \multicolumn{2}{|r|}{ Men } & \multicolumn{2}{|r|}{ Women } \\
\hline & Cases & $\mathrm{HR}(95 \% \mathrm{Cl})^{\mathrm{a} b}$ & Cases & $\mathrm{HR}(95 \% \mathrm{Cl})^{\mathrm{abc}}$ \\
\hline Overall & 12184 & $1.02(0.95-1.10)$ & 7071 & $0.93(0.86-1.01)$ \\
\hline \multicolumn{5}{|l|}{ Cigarette smoking status ${ }^{d}$} \\
\hline Never & 1367 & $0.99(0.82-1.20)$ & 3970 & $0.93(0.85-1.03)$ \\
\hline Former & 6084 & $1.05(0.96-1.15)$ & 1490 & $0.87(0.72-1.04)$ \\
\hline Current & 4733 & $0.98(0.85-1.12)$ & 1611 & $0.97(0.81-1.18)$ \\
\hline $\mathrm{P}_{\text {interaction }}{ }^{\mathrm{e}}$ & & 0.801 & & 0.363 \\
\hline \multicolumn{5}{|l|}{ Alcohol consumption ${ }^{f}$} \\
\hline $0 \mathrm{~g} /$ day & 1488 & $1.03(0.86-1.24)$ & 2166 & $0.91(0.78-1.05)$ \\
\hline$>0-<15.0 \mathrm{~g} /$ day & 5616 & 1.07 (0.97 - 1.19) & 3838 & $0.97(0.88-1.07)$ \\
\hline$\geq 15.0 \mathrm{~g} /$ day & 5080 & $0.95(0.84-1.07)$ & 1067 & $0.80(0.63-1.00)$ \\
\hline $\mathrm{P}_{\text {interaction }}{ }^{\mathrm{e}}$ & & 0.344 & & 0.532 \\
\hline \multicolumn{5}{|l|}{ Body mass index ${ }^{g}$} \\
\hline$\geq 18.5-<25.0 \mathrm{~kg} / \mathrm{m}^{2}$ & 6310 & $0.99(0.89-1.09)$ & 3699 & $0.91(0.82-1.01)$ \\
\hline$\geq 25.0 \mathrm{~kg} / \mathrm{m}^{2}$ & 5826 & $1.07(0.96-1.19)$ & 3293 & $0.93(0.82-1.04)$ \\
\hline$P_{\text {interaction }}{ }^{\mathrm{e}}$ & & 0.263 & & 0.740 \\
\hline \multicolumn{5}{|l|}{ Highest level of education ${ }^{\mathrm{h}}$} \\
\hline Primary school or lower vocational & 5430 & $0.96(0.86-1.08)$ & 3742 & $0.87(0.79-0.97)$ \\
\hline Secondary school or medium vocational & 4319 & $1.04(0.93-1.16)$ & 2643 & $0.96(0.84-1.10)$ \\
\hline Higher vocational or university & 2435 & $1.19(1.01-1.41)$ & 686 & $1.19(0.91-1.57)$ \\
\hline $\mathrm{P}_{\text {interaction }} \mathrm{e}^{\mathrm{e}}$ & & 0.049 & & 0.408 \\
\hline \multicolumn{5}{|l|}{ Family history of cancer ${ }^{\mathrm{i}}$} \\
\hline No & 6267 & $1.06(0.96-1.17)$ & 3375 & $0.94(0.84-1.04)$ \\
\hline Yes & 5917 & $0.98(0.89-1.09)$ & 3696 & $0.94(0.84-1.04)$ \\
\hline $\mathrm{P}_{\text {interaction }}{ }^{\mathrm{e}}$ & & 0.261 & & 0.785 \\
\hline
\end{tabular}

Abbreviation: aMEDr, alternate Mediterranean diet score without the alcohol component

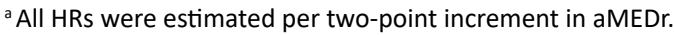

${ }^{\mathrm{b}}$ Adjusted for age at baseline (years), cigarette smoking status (never, former, current), cigarette smoking frequency (cigarettes smoked per day, centered), cigarette smoking duration (years, centered), body mass index $\left(<18.5, \geq 18.5-<25.0, \geq 25.0-<30.0, \geq 30.0 \mathrm{~kg} / \mathrm{m}^{2}\right)$, height $(\mathrm{cm})$, alcohol consumption $(0,>0-<5, \geq 5-<15, \geq 15-<30$, $\geq 30 \mathrm{~g} /$ day), daily energy intake (kcal), highest level of education (primary school or lower vocational, secondary school or medium vocational, higher vocational or university), non-occupational physical activity $(\leq 30,>30-\leq 60$, $>60-\leq 90,>90 \mathrm{~min} /$ day), and family history of cancer (no, yes).

${ }^{c}$ Analyses conducted among women were additionally adjusted for age at menarche $(\leq 12,13-14,15-16, \geq 17$ years), parity (nulliparous, $1-2, \geq 3$ children), age at first birth ( $<25, \geq 25$ years), age at menopause ( $\leq 44,45-49$, $50-54, \geq 55$ years), oral contraceptive use (never, ever), and use of postmenopausal hormone replacement therapy (never, ever).

${ }^{\mathrm{d}}$ Not adjusted for cigarette smoking status. 
Table 8.4 (footnotes, continued)

e $\mathrm{P}$-values for interaction were obtained by testing the statistical significance of interaction terms between aMEDr and the stratifying covariates in multivariable-adjusted models.

${ }^{\mathrm{f}}$ Not adjusted for alcohol consumption.

${ }^{8}$ Not adjusted for body mass index.

${ }^{\mathrm{h}}$ Not adjusted for highest level of education.

iNot adjusted for family history of cancer.

(partly) be responsible for the inconsistent findings concerning overall cancer risk.

Results of the present study indicated that the inverse association between MD adherence and overall cancer risk, if present, might be restricted to women. In line with our findings, slightly stronger inverse associations were observed in female participants of EPIC-Greece, though the interaction by sex did not reach statistical significance [9]. Cancers arising in men and women may etiologically differ. The sex-specific levels of sex hormones may influence tumor development and could therefore potentially modulate the association of dietary factors with cancer risk [39-43]. Apart from other factors, sex-related differences may also exist in exposure levels to risk factors and carcinogen metabolism [39, 41-43]. Furthermore, the disparate associations of MD adherence with commonly diagnosed sex-specific cancers (i.e., postmenopausal breast and prostate cancer) are likely to have contributed to the heterogeneous relations of MD adherence with overall cancer risk for men and women. It should be noted that other studies did not observe clear differences in associations between the sexes $[10,13]$, stressing the importance of additional research on this topic.

Associations with MD adherence among women in our study appeared comparable for overall cancer risk and risks of cancer subgroups defined by the presence of a relation with tobacco smoking, obesity, or alcohol consumption. In contrast to our findings for women, significant heterogeneity was observed in all subgroup comparisons in men. However, associations with MD adherence did not reach statistical significance for any of the subgroups in men and the differences did not seem to be relevant. The statistical power in our study was high, especially for men, which increased the likelihood for small and irrelevant differences to become statistically significant. Additionally, one should realize that the distribution of the individual cancer types differs between the subgroups in men and women, and that in certain subgroups a substantial proportion can be comprised by sex-specific cancers. Regarding cancers related vs. not related to obesity and alcohol consumption, similar results were obtained in previous studies $[10,13]$. The inverse association with MD adherence was stronger for smoking-related cancers compared to cancers not related to tobacco smoking in the total EPIC cohort [10], whereas the opposite was observed in the Greek EPIC cohort [9]. Furthermore, associations did not seem to differ in a Swedish cohort [13]. These contrasting findings may have resulted from differences in the classification of cancer types as being related to tobacco smoking or not. For example, while cancers of the colorectum/large bowel were classified as being smoking-related in the studies by Couto et al. [10] and Bodén et al. [13], they were considered not being related to smoking in the study by Benetou et al. [9]. Moreover, the subgroup of cancers not being related to tobacco smoking constituted all cancers not classified as being related to smoking in one study [13], whereas the two other studies selected specific cancer types $[9,10]$.

The cancer-preventive effect of the MD seems biologically plausible. The high intake of dietary antioxidants in the MD (e.g., polyphenols and vitamins from plant foods and olive oil) and the resulting higher total antioxidant capacity that has been associated with adherence to 
this dietary pattern may defend the body against the DNA-damaging effects of free radicals and other oxidants [44-46]. Moreover, the anti-inflammatory effects of polyphenols and the favorable fatty acid profile of the MD (high in anti-inflammatory omega-3 polyunsaturated fatty acids) may reduce inflammation $[45,47]$. Several additional mechanisms have been proposed for the cancer-preventive effect of the MD, which were among others related to body weight regulation [48] and the low consumption of red and processed meats [30, 46].

Important strengths of the NLCS include the large sample size, prospective design, and nearly complete follow-up of 20.3 years, which make information and selection biases unlikely. We had adequate statistical power to perform sex-specific analyses for overall cancer risk as well as risks of cancer subgroups defined by relations with three major cancer risk factors. The possibility of residual confounding was minimized through our comprehensive adjustment for cigarette smoking and other potential confounders, including reproductive factors in women. Limitations of our study include the lack of updated dietary information during follow-up and possible measurement errors in the exposure assessment, which may have attenuated some associations. The use of cohort-specific cut-offs in the assessment of MD adherence may pose a final weakness of our study. Subjects with high aMEDr values in the non-Mediterranean study population of the NLCS could potentially be classified in intermediate or low adherence categories in populations with higher intakes of typically Mediterranean foods. Nevertheless, our results did suggest that, also in the nonMediterranean population of the Netherlands, adherence to a more Mediterranean-like dietary pattern might reduce overall cancer risk in women.

\section{Conclusions}

Higher MD adherence seemed to be associated with a reduced overall cancer risk in women in our large prospective cohort study. However, the inverse associations did generally not reach statistical significance in multivariable-adjusted analyses. Associations of MD adherence with subgroups of cancer defined by relations with tobacco smoking, obesity, and alcohol consumption, closely resembled the results obtained for overall cancer risk in women. In men, no associations were observed between MD adherence and risk of overall cancer or any of the cancer subgroups. This does not exclude the possibility of a beneficial effect of MD adherence on specific cancer (sub)types in men, as we have already shown in previous NLCS analyses [28, 49].

Even though the association of MD adherence with overall cancer risk is comprised of a combination of potentially diverging associations with individual cancer (sub)types, overall cancer risk is still an interesting end point for epidemiological studies, particularly to evaluate the potential of the MD as a dietary strategy for cancer prevention. Our observation of a better performance of the MD score variant without alcohol is also important in the latter perspective. Because alcohol consumption is associated with an increased risk of several cancer types, we would prefer a Mediterranean-type diet that does not incorporate moderate alcohol consumption. 


\section{References}

1. Bray F, Ferlay J, Soerjomataram I, Siegel RL, Torre LA, Jemal A. Global cancer statistics 2018: GLOBOCAN estimates of incidence and mortality worldwide for 36 cancers in 185 countries. CA Cancer J Clin. 2018;68(6):394-424.

2. Lanting $\mathrm{Cl}$, de Vroome EM, Elias SG, van den Brandt PA, van Leeuwen FE, Kampman E, et al. [Contribution of lifestyle factors to cancer: secondary analysis of Dutch data over 2010 and a projection for 2020]. Ned Tijdschr Geneeskd. 2014;159:A8085 (in Dutch).

3. Willett WC, Sacks F, Trichopoulou A, Drescher G, Ferro-Luzzi A, Helsing E, et al. Mediterranean diet pyramid: a cultural model for healthy eating. Am J Clin Nutr. 1995;61(6 Suppl):1402S-6S.

4. Trichopoulou A, Lagiou P. Healthy traditional Mediterranean diet: an expression of culture, history, and lifestyle. Nutr Rev. 1997;55(11 Pt 1):383-9.

5. Steck SE, Guinter M, Zheng J, Thomson CA. Index-based dietary patterns and colorectal cancer risk: a systematic review. Adv Nutr. 2015;6(6):763-73.

6. van den Brandt PA, Schulpen M. Mediterranean diet adherence and risk of postmenopausal breast cancer: results of a cohort study and meta-analysis. Int J Cancer. 2017;140(10):2220-31.

7. Schwingshackl L, Schwedhelm C, Galbete C, Hoffmann G. Adherence to Mediterranean Diet and Risk of Cancer: An Updated Systematic Review and Meta-Analysis. Nutrients. 2017;9(10).

8. Cheng $S$, Zheng $Q$, Ding G, Li G. Mediterranean dietary pattern and the risk of prostate cancer: A meta-analysis. Medicine (Baltimore). 2019;98(27):e16341.

9. Benetou V, Trichopoulou A, Orfanos P, Naska A, Lagiou P, Boffetta P, et al. Conformity to traditional Mediterranean diet and cancer incidence: the Greek EPIC cohort. $\mathrm{Br} \mathrm{J}$ Cancer. 2008;99(1):191-5.

10. Couto E, Boffetta P, Lagiou P, Ferrari P, Buckland G, Overvad K, et al. Mediterranean dietary pattern and cancer risk in the EPIC cohort. Br J Cancer. 2011;104(9):1493-9.

11. Galbete C, Kroger J, Jannasch F, Iqbal K, Schwingshackl L, Schwedhelm C, et al. Nordic diet, Mediterranean diet, and the risk of chronic diseases: the EPIC-Potsdam study. BMC Med. 2018;16(1):99.

12. Lavalette C, Adjibade M, Srour B, Sellem L, Fiolet T, Hercberg S, et al. Cancer-Specific and General Nutritional Scores and Cancer Risk: Results from the Prospective NutriNet-Sante Cohort. Cancer Res. 2018;78(15):4427-35.

13. Boden S, Myte R, Wennberg M, Harlid S, Johansson I, Shivappa N, et al. The inflammatory potential of diet in determining cancer risk; A prospective investigation of two dietary pattern scores. PLoS One. 2019;14(4):e0214551.

14. van den Brandt PA, Goldbohm RA, van 't Veer P, Volovics A, Hermus RJ, Sturmans F. A largescale prospective cohort study on diet and cancer in The Netherlands. J Clin Epidemiol. 1990;43(3):28595.

15. van den Brandt PA, Schouten LJ, Goldbohm RA, Dorant E, Hunen PM. Development of a record linkage protocol for use in the Dutch Cancer Registry for Epidemiological Research. Int J Epidemiol. 1990;19(3):553-8.

16. Goldbohm RA, van den Brandt PA, Brants HA, van 't Veer P, Al M, Sturmans F, et al. Validation of a dietary questionnaire used in a large-scale prospective cohort study on diet and cancer. Eur J Clin Nutr. 1994;48(4):253-65.

17. Volovics A, van den Brandt PA. Methods for the analyses of case-cohort studies. Biometrical J. 1997;39(2):195-214.

18. Prentice RL. A case-cohort design for epidemiologic cohort studies and disease prevention trials. Biometrika. 1986;73(1):1-11.

19. Secretan B, Straif K, Baan R, Grosse Y, El Ghissassi F, Bouvard V, et al. A review of human carcinogens--Part E: tobacco, areca nut, alcohol, coal smoke, and salted fish. Lancet Oncol. 2009;10(11):1033-4.

20. McKenzie F, Biessy C, Ferrari P, Freisling H, Rinaldi S, Chajes V, et al. Healthy Lifestyle and Risk of Cancer in the European Prospective Investigation Into Cancer and Nutrition Cohort Study. Medicine (Baltimore). 2016;95(16):e2850.

21. Lauby-Secretan B, Scoccianti C, Loomis D, Grosse Y, Bianchini F, Straif K, et al. Body Fatness and Cancer--Viewpoint of the IARC Working Group. N Engl J Med. 2016;375(8):794-8. 
22. Goldbohm RA, van 't Veer P, van den Brandt PA, van 't Hof MA, Brants HA, Sturmans F, et al. Reproducibility of a food frequency questionnaire and stability of dietary habits determined from five annually repeated measurements. Eur J Clin Nutr. 1995;49(6):420-9.

23. NEVO table. Dutch food composition table 1986-1987. The Hague, the Netherlands: Voorlichtingsbureau voor de Voeding; 1986.

24. Fung TT, McCullough ML, Newby PK, Manson JE, Meigs JB, Rifai N, et al. Diet-quality scores and plasma concentrations of markers of inflammation and endothelial dysfunction. Am J Clin Nutr. 2005;82(1):163-73.

25. Mitrou PN, Kipnis V, Thiebaut AC, Reedy J, Subar AF, Wirfalt E, et al. Mediterranean dietary pattern and prediction of all-cause mortality in a US population: results from the NIH-AARP Diet and Health Study. Arch Intern Med. 2007;167(22):2461-8.

26. Trichopoulou A, Kouris-Blazos A, Wahlqvist ML, Gnardellis C, Lagiou P, Polychronopoulos E, et al. Diet and overall survival in elderly people. BMJ. 1995;311(7018):1457-60.

27. Trichopoulou A, Costacou T, Bamia C, Trichopoulos D. Adherence to a Mediterranean diet and survival in a Greek population. N Engl J Med. 2003;348(26):2599-608.

28. Schulpen $M$, van den Brandt PA. Adherence to the Mediterranean diet and risk of lung cancer in the Netherlands Cohort Study. Br J Nutr. 2018;119(6):674-84.

29. Bagnardi V, Rota M, Botteri E, Tramacere I, Islami F, Fedirko V, et al. Alcohol consumption and site-specific cancer risk: a comprehensive dose-response meta-analysis. Br J Cancer. 2015;112(3):58093.

30. World Cancer Research Fund / American Institute for Cancer Research. Diet, nutrition, physical activity and cancer: a global perspective. Continuous update project expert report 2018. 2018. Available from: https://www.wcrf.org/dietandcancer.

31. Lin DY, Wei LJ. The Robust Inference for the Cox Proportional Hazards Model. J Am Stat Assoc. 1989;84(408):1074-8.

32. Grambsch PM, Therneau TM. Proportional Hazards Tests and Diagnostics Based on Weighted Residuals. Biometrika. 1994;81(3):515-26.

33. Akaike H. A new look at the statistical model identification. IEEE Transactions on automatic control. 1974;AC-19(6):716-23.

34. de Vogel S, Bongaerts BW, Wouters KA, Kester AD, Schouten LJ, de Goeij AF, et al. Associations of dietary methyl donor intake with MLH1 promoter hypermethylation and related molecular phenotypes in sporadic colorectal cancer. Carcinogenesis. 2008;29(9):1765-73.

35. Wacholder S, Gail MH, Pee D, Brookmeyer R. Alternative Variance and Efficiency Calculations for the Case-Cohort Design. Biometrika. 1989;76(1):117-23.

36. de Lorgeril M, Salen P, Martin JL, Monjaud I, Boucher P, Mamelle N. Mediterranean dietary pattern in a randomized trial: prolonged survival and possible reduced cancer rate. Arch Intern Med. 1998;158(11):1181-7.

37. Schulpen M, van den Brandt PA. Adherence to the Mediterranean Diet and Risks of Prostate and Bladder Cancer in the Netherlands Cohort Study. Cancer Epidemiol Biomarkers Prev. 2019;28(9):1480-8.

38. Schulpen $\mathrm{M}$, van den Brandt PA. Mediterranean diet adherence and risk of colorectal cancer: the prospective Netherlands Cohort Study. Eur J Epidemiol. 2019.

39. Dorak MT, Karpuzoglu E. Gender differences in cancer susceptibility: an inadequately addressed issue. Front Genet. 2012;3:268.

40. Kim SE, Paik HY, Yoon H, Lee JE, Kim N, Sung MK. Sex- and gender-specific disparities in colorectal cancer risk. World J Gastroenterol. 2015;21(17):5167-75.

41. Dobruch J, Daneshmand S, Fisch M, Lotan Y, Noon AP, Resnick MJ, et al. Gender and Bladder Cancer: A Collaborative Review of Etiology, Biology, and Outcomes. Eur Urol. 2016;69(2):300-10.

42. Kim HI, Lim H, Moon A. Sex Differences in Cancer: Epidemiology, Genetics and Therapy. Biomol Ther (Seoul). 2018;26(4):335-42.

43. Stapelfeld C, Dammann C, Maser E. Sex-specificity in lung cancer risk. Int J Cancer. 2019.

44. Pitsavos C, Panagiotakos DB, Tzima N, Chrysohoou C, Economou M, Zampelas A, et al. Adherence to the Mediterranean diet is associated with total antioxidant capacity in healthy adults: the ATTICA study. Am J Clin Nutr. 2005;82(3):694-9.

45. Brill JB. The Mediterranean diet and your health. Am J Lifestyle Med. 2009;3(1):44-56. 
46. Verberne L, Bach-Faig A, Buckland G, Serra-Majem L. Association between the Mediterranean diet and cancer risk: a review of observational studies. Nutr Cancer. 2010;62(7):860-70.

47. Rahman I, Biswas SK, Kirkham PA. Regulation of inflammation and redox signaling by dietary polyphenols. Biochem Pharmacol. 2006;72(11):1439-52.

48. Romaguera D, Norat T, Vergnaud AC, Mouw T, May AM, Agudo A, et al. Mediterranean dietary patterns and prospective weight change in participants of the EPIC-PANACEA project. Am J Clin Nutr. 2010;92(4):912-21.

49. Schulpen $\mathrm{M}$, Peeters PH, van den Brandt PA. Mediterranean diet adherence and risk of esophageal and gastric cancer subtypes in the Netherlands Cohort Study. Gastric Cancer. 2019;22(4):663-74. 


\section{Chapter 9}

\section{General discussion}

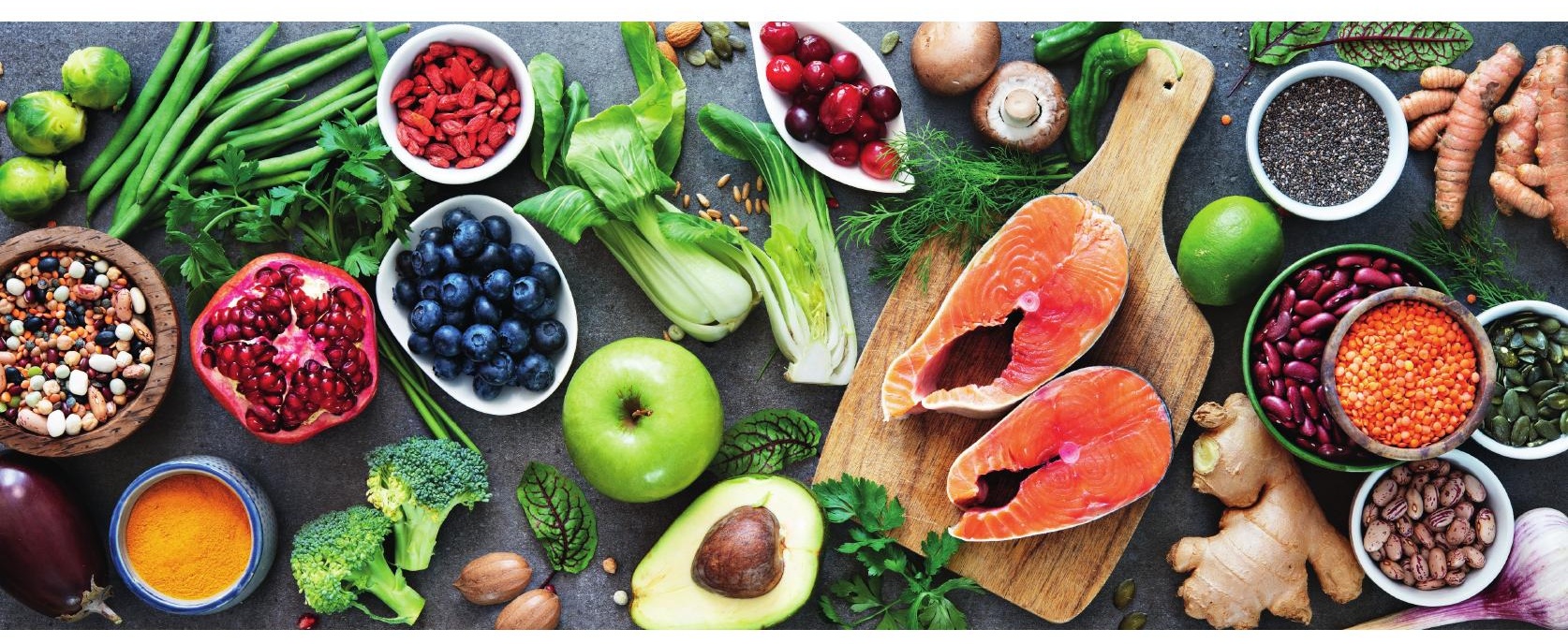


The principal aim of this thesis was to evaluate the association of Mediterranean diet (MD) adherence with risks of overall cancer and specific cancer types in the Netherlands in a prospective setting. This aim was primarily studied within the Netherlands Cohort Study on Diet and Cancer (NLCS). However, for pancreatic cancer, we additionally used data collected as part of the Dutch cohort of the European Prospective Investigation into Cancer and Nutrition (EPIC-NL). The relative level of MD adherence was assessed using two $a$ priori defined MD scores: the alternate Mediterranean diet score (aMED) and the modified Mediterranean diet score (mMED). Although moderate consumption of wine during meals is considered beneficial in the traditional MD, alcohol consumption has been related to increased risks of several types of cancer. Therefore, we created score variants without the alcohol component (aMEDr and mMEDr, respectively) and compared these to the original scores (including alcohol component). Special attention was paid to potential heterogeneity of associations across the sexes and subtypes of specific cancer sites.

In this chapter, we will first provide a summary of the most important findings of this thesis. Subsequently, we will try to put these findings into perspective by relating them to previously published prospective research evaluating the effect of a priori defined MD adherence and discussing some methodological considerations. Furthermore, the implications of our findings for policymakers and the Dutch society will be addressed. We will end this chapter with our recommendations for future research.

\section{Summary of the main findings}

In this section, we will highlight the most important results of this thesis. For most cancer sites, equal or better model fits were observed when MD adherence was assessed using aMEDr as opposed to mMEDr. Therefore, we will focus on results obtained for aMEDr in the description and interpretation of our main findings. Additionally, we prefer the aMED variant without alcohol (aMEDr), because alcohol consumption is a well-established risk factor for multiple cancer types [1-3]. The most important findings of this thesis are summarized in Table 9.1. For associations between mMEDr and the investigated cancer sites, we refer to the corresponding chapters of this thesis.

First, we investigated the association of MD adherence with lung cancer incidence (Chapter 2), showing non-significant inverse associations when comparing the highest to the lowest aMEDr category in both men and women. The inverse associations appeared to be strongest in women and never smokers ( $\mathrm{p}_{\text {interaction }}>0.05$ in both cases). Although heterogeneity tests did not reach statistical significance, the strength of the associations seemed to vary somewhat across the histological lung cancer subtypes, especially in men.

Furthermore, higher adherence to the MD was associated with a significantly reduced risk of estrogen receptor (ER) negative postmenopausal breast cancer in the NLCS, as we showed in Chapter 3. Weaker, and not statistically significant, inverse associations were observed for risks of total and ER positive postmenopausal breast cancer. Similar patterns were observed for progesterone receptor (PR) and combined ER/PR subtypes. Additionally, we performed meta-analyses including our results and those of previously published cohort studies. Results of these meta-analyses were consistent with the NLCS findings.

Different etiologies have been suggested for subtypes of esophageal and gastric cancer. Therefore, subtype-specific associations of these cancer sites with MD adherence were evaluated in Chapter 4. In both sexes, higher MD adherence was associated with decreased risks of gastric cancer subtypes [i.e., gastric cardia adenocarcinoma (GCA) and gastric non- 
cardia adenocarcinoma (GNCA)]. However, the inverse associations were only statistically significant in men. For esophageal squamous cell carcinoma (ESCC) risk, associations significantly differed between the sexes. A statistically significant inverse association was observed in men, whereas there was no evidence of an association in women. MD adherence was not associated with esophageal adenocarcinoma (EAC) risk in both men and women.

In Chapter 5, the association between MD adherence and pancreatic cancer incidence was examined by pooling results of the NLCS and EPIC-NL. In these analyses, MD adherence was not significantly associated with risk of microscopically confirmed pancreatic cancer (MCPC) in both sexes. Our findings suggested that the association between MD adherence and MCPC risk might be modified by smoking status. Weak and non-significant inverse associations were observed in never smokers, but not in ever smokers. The overall conclusion was similar when we also included cases that were not microscopically confirmed in the analyses.

Similarly, we observed no significant association between MD adherence and colorectal cancer risk (Chapter 6). Associations were absent for all anatomical subsites (i.e., colon, proximal colon, distal colon, and rectum) in both men and women.

In Chapter 7, aMEDr was not associated with the risk of advanced prostate cancer. However, a statistically significant positive association was observed for nonadvanced prostate cancer risk. Possibly, nonadvanced prostate tumors have a higher chance of being detected in higher educated men with a more health-conscious lifestyle and greater adherence to the $M D$, who may be more aware of prostate cancer and more likely to seek care or attend screening programs. For bladder cancer risk (Chapter 7), we did not observe an association with MD adherence, regardless of the malignancy grade at diagnosis.

Finally, the association of MD adherence with overall cancer incidence was evaluated in Chapter 8. Although not statistically significant, increasing MD adherence seemed to be associated with a decreased risk of cancer in women. However, there was no evidence of an inverse association in men. In addition, we compared associations with MD adherence for subgroups of cancers related vs. not related to tobacco smoking, obesity, and alcohol consumption, and concluded that these did not relevantly differ, irrespective of sex. In men, heterogeneity tests were significant for all subgroup comparisons made, but differences were small and probably reached statistical significance because of the large number of cases.

For the findings described above, MD adherence was assessed using aMEDr, which does not include alcohol. Largely similar results were obtained when we used the original aMED including alcohol component. Generally, inclusion of alcohol in the MD score either did not affect the model performance or resulted in a worse model fit.

\section{Interpretation of the main findings}

In our analyses conducted in the Dutch population, MD adherence seemed to be associated with (non-significantly) reduced risks of some cancer types (i.e., cancers of the lung and breast, ESCC, GCA, and GNCA). Many of these cancer (sub)types have a relatively poor prognosis, underlining the importance of the development of successful preventive strategies for these cancer sites.

Closer adherence to an a priori defined MD has consistently been associated with a decreased lung cancer risk in prospective studies [4-7]. The largest cohort evaluating this relation was the National Institutes of Health-American Association of Retired Persons (NIH-AARP) Diet and Health Study (9272 incident lung cancer cases among 460770 eligible participants) [5], 
Table 9.1 Summary of the main findings of this thesis with respect to the association between Mediterranean diet adherence and cancer risk in the Netherlands ${ }^{a} b$

\begin{tabular}{|c|c|c|c|c|c|}
\hline & \multicolumn{2}{|c|}{ Men } & \multicolumn{2}{|c|}{ Women } & \multirow[t]{3}{*}{ Chapter } \\
\hline & - Alcohol & + Alcohol & - Alcohol & + Alcohol & \\
\hline & aMEDr & aMED & aMEDr & aMED & \\
\hline Lung & $(-)$ & $(-)$ & $(-)$ & $(-)$ & 2 \\
\hline - $\quad$ Adenocarcinoma & 0 & & $(-)$ & & \\
\hline - $\quad$ Squamous cell carcinoma & $(-)$ & & $(-)$ & & \\
\hline - $\quad$ Small cell carcinoma & $(-)$ & & $(-)$ & & \\
\hline - $\quad$ Large cell carcinoma & 0 & & 0 & & \\
\hline Breast (postmenopausal)c & & & $(-)$ & $(-)$ & 3 \\
\hline - $\quad E R+$ & & & $(-)$ & & \\
\hline$-\quad$ ER- & & & - & & \\
\hline Esophagus & & & & & 4 \\
\hline - $\quad$ ESCC & - & - & 0 & 0 & \\
\hline EAC & 0 & 0 & 0 & 0 & \\
\hline Stomach & & & & & 4 \\
\hline - $\quad$ GCA & - & $(-)$ & $(-)$ & $(-)$ & \\
\hline - $\quad$ GNCA & - & - & $(-)$ & $(-)$ & \\
\hline Pancreas (MCPC) & 0 & 0 & 0 & 0 & 5 \\
\hline Colorectum & 0 & 0 & 0 & 0 & 6 \\
\hline - $\quad$ Colon & 0 & 0 & 0 & 0 & \\
\hline - $\quad$ Rectum & 0 & 0 & 0 & 0 & \\
\hline Prostate & + & + & & & 7 \\
\hline - $\quad$ Nonadvanced & + & + & & & \\
\hline - $\quad$ Advanced & 0 & 0 & & & \\
\hline Bladder $^{d}$ & 0 & 0 & 0 & 0 & 7 \\
\hline - $\quad$ Noninvasive & 0 & 0 & 0 & 0 & \\
\hline - Invasive & 0 & 0 & 0 & 0 & \\
\hline Overall cancer & 0 & 0 & $(-)$ & $(-)$ & 8 \\
\hline
\end{tabular}

Abbreviations: aMEDr, alternate Mediterranean diet score without the alcohol component; aMED, alternate Mediterranean diet score; ER+, estrogen receptor positive; ER-, estrogen receptor negative; ESCC, esophageal squamous cell carcinoma; EAC, esophageal adenocarcinoma; GCA, gastric cardia adenocarcinoma; GNCA, gastric non-cardia adenocarcinoma; MCPC, microscopically confirmed pancreatic cancer; NLCS, Netherlands Cohort Study; EPIC-NL, the Dutch cohort of the European Prospective Investigation into Cancer and Nutrition; PR+, progesterone receptor positive; PR-, progesterone receptor negative

${ }^{a}$ Associations were estimated within the NLCS for all cancer sites. For pancreatic cancer, the presented associations were obtained by pooling results from the NLCS and EPIC-NL.

${ }^{b}$ - inverse association; (-) inverse association, not statistically significant; 0 null association; (+) positive association, not statistically significant; + positive association.

c Patterns for the PR+/PR- and the combined ER/PR subtypes resembled the pattern that was observed for the ER+/ER- subtypes.

${ }^{\mathrm{d}}$ The presented associations for bladder cancer risk were determined in men and women combined. 
which showed that MD adherence was associated with a significantly reduced lung cancer risk in both sexes in the US. Subtype-specific analyses revealed that the inverse association was statistically significant for adenocarcinoma and squamous cell carcinoma, but not for small cell carcinoma. Likewise, the strength of the association with MD adherence also seemed to vary across the histological subtypes in this thesis. Additionally, our results indicated that the potentially beneficial effect of MD adherence on lung cancer risk might be slightly more pronounced in women compared to men. The small number of incident lung cancer cases in the other cohorts possibly hampered detailed evaluations of subtype- or sex-specific associations. Tobacco smoking is by far the strongest risk factor for lung cancer [1]. Because smoking often coincides with an unhealthy diet and lifestyle, residual confounding by tobacco smoking is a major concern in the analysis of dietary factors and lung cancer risk. It is unlikely that the non-significant inverse associations of MD adherence with lung cancer risk that we observed can fully be attributed to residual confounding by tobacco smoking. We adjusted extensively for potential confounding by cigarette smoking through the inclusion of smoking status, frequency, and duration in our multivariable models. Moreover, associations were also in the inverse direction when we only considered NLCS participants who had never smoked.

Furthermore, our results suggested that MD adherence might reduce postmenopausal breast cancer risk, particularly of the ER negative subtype. The effect of a MD supplemented with either extra-virgin olive oil or mixed nuts compared to a low-fat control diet on postmenopausal breast cancer risk was evaluated in the randomized Prevención con Dieta Mediterránea trial [PREDIMED, 35 incident breast cancer cases diagnosed among 4282 Spanish women with high cardiovascular disease (CVD) risk] [8]. After a median followup of 4.8 years, incidence rates of postmenopausal breast cancer turned out to be (nonsignificantly) lower in the MD groups. Additionally, the association between a priori defined MD adherence and breast cancer risk has been investigated in a number of cohort studies $[7,9-17]$. For an elaborate discussion of the literature concerning MD adherence and breast cancer risk we refer to Chapter 3 of this thesis. Since we wrote this chapter, several additional prospective investigations have been published [7, 15-17], of which the study by Petimar et al. [17] is of particular interest. This study showed that MD adherence was inversely associated with breast cancer risk in US women with a family history of breast cancer. Although the inverse associations were not statistically significant for all hormone receptor subtypes, they seemed slightly more pronounced for ER negative breast cancers, with the strongest inverse relation being observed for triple-negative breast cancer [17]. Considering the prospective cohort evidence in its totality, one may conclude that the inverse association of MD adherence with breast cancer risk, if present, seems to be restricted to postmenopausal women. Genetic and early life factors may play a more important role in premenopausal disease $[10,12,18,19]$. Despite some heterogeneity, findings for the hormone receptor subtypes indicated that the potentially protective effect of MD adherence might be more pronounced for ER negative breast cancers. Possibly, (weak) relations of dietary factors with ER negative breast cancers are easier to detect, because of the strong influence of hormonal factors on ER positive breast cancers [9, 12].

For esophageal cancer, we found MD adherence to be significantly inversely associated with ESCC risk among men, whereas no association was observed with EAC risk, regardless of sex. The heterogeneous associations for ESCC and EAC risk were not unexpected, as varying risk factors and incidence trends suggest different etiological backgrounds [20, 21]. In line with this thesis, a similar pattern for the esophageal cancer subtypes was observed in the only other cohort study (NIH-AARP) conducted on this topic [22]. Additionally, the significantly reduced ESCC risk upon higher levels of MD adherence in the NIH-AARP cohort seemed to 
be restricted to men as well.

Concerning the gastric cancer subtypes, findings of prospective studies are inconclusive $[22,23]$. In the NIH-AARP cohort [22], MD adherence was not significantly associated with a reduced risk of either subtype. Nonetheless, the results were indicative of a possible inverse association with GNCA risk. Although both gastric cancer subtypes were inversely associated with MD adherence in EPIC [23], statistical significance was only reached for GCA risk. The EPIC cohort involves centers from ten European countries of which three are located in the Mediterranean region (i.e., Greece, Italy, and Spain) [24]. In our analyses of the NLCS, the (non-significant) inverse associations with MD adherence seemed to be of roughly comparable strength for GCA and GNCA risk. Of note, neither our study nor the NIH-AARP and EPIC studies were able to correct for Helicobacter pylori infection, which is an important risk factor for GNCA [25] and could potentially have confounded the results. When examining the relation between dietary patterns and (upper) gastrointestinal cancer risk, potential bias resulting from reversed causation should be carefully considered. Already before their diagnosis, dietary habits of cases may have been influenced by preclinical disease symptoms. When these symptoms would have led to a decreased consumption of Mediterranean foods (e.g., vegetables, fruits, or nuts), it may erroneously be concluded that MD adherence is protective against gastrointestinal cancers. Results of cohort studies are less likely to be affected by reversed causation than results of case-control studies. Because exclusion of the first two years of follow-up did not essentially alter our findings for esophageal and gastric cancer risk, the inverse associations that we observed probably have not resulted from reversed causation. Moreover, results of the NIH-AARP and EPIC cohorts were also largely comparable after excluding the first few years of follow-up in a lag analysis $[22,23]$.

MD adherence was not associated with a decreased incidence of cancers of the pancreas, colorectum (any subsite), prostate (advanced), and bladder in this thesis. However, previously published prospective studies did suggest a potentially beneficial effect of $a$ priori defined MD adherence on colorectal and bladder cancer risk.

Even though overall findings for colorectal cancer risk have been mixed, greater MD adherence has rather consistently been associated with a reduced colorectal cancer risk in men [26-31]. For women, on the other hand, previous results have generally not been supportive of an inverse association [7, 26, 28-33]. Associations with MD adherence may differ between the anatomical subsites of colorectal cancer as well. Besides the potentially distinct etiological backgrounds of these tumors, exposures to (carcinogens in) fecal matter may vary across the length of the colorectum [34, 35]. Accordingly, in male participants of the US-based NIH-AARP [26] and Health Professionals Follow-up Study (HPFS, [31]) cohorts, inverse associations with MD adherence seemed strongest for tumors arising in the distal colon and rectum. In our analyses, we did not observe an inverse relation of MD adherence with any of the colorectal cancer subsites in both men and women. Perhaps, the relatively modest contrasts in absolute food intakes between participants with high and low MD adherence in the homogeneous NLCS study population did not suffice to enable detection of the rather weak inverse association of this dietary pattern with colorectal cancer risk that seems to be present in men. Nevertheless, MD adherence was associated with (non-) significantly reduced risks of lung cancer, ESCC, GCA, and GNCA in male NLCS participants, as we have described above, which suggests adequate exposure contrast.

Concerning bladder cancer, MD adherence was not significantly associated with urothelial cell carcinoma (UCC) risk in two initial cohort studies, which were conducted in Europe and Australia, respectively $[36,37]$. However, effect estimates pointed towards an inverse 
association, particularly for invasive UCC. A significant inverse association between MD adherence and bladder cancer risk was reported in a more recent analysis of 13 prospective cohort studies [38]. A limitation of all three previous analyses is the level of adjustment for cigarette smoking, i.e., the most important bladder cancer risk factor [39], which was not as comprehensive, and could potentially have accounted for the differential findings compared to our study. Moreover, the inverse associations were restricted to (former and) current smokers in two of the three studies [36, 37], and thus may have resulted from residual confounding by cigarette smoking.

The currently available prospective evidence does not point towards a strong beneficial influence of MD adherence on pancreatic and prostate cancer risk, which is in agreement with our analyses.

The relation between a priori defined MD adherence and pancreatic cancer incidence has been evaluated in three cohort studies (2 European, 1 US), all showing no significant association [7, 40,41]. Comparable to the EPIC-wide result, MD adherence was also not associated with a significantly decreased pancreatic cancer risk in the southern European countries of the EPIC cohort in subgroup analyses [41]. Furthermore, the use of cohort-wide instead of country-specific tertiles in the assessment of MD adherence did not appreciably affect the associations [41].

Considering prostate cancer, advanced tumors at diagnosis comprise the most informative end point. In contrast to the advanced subtype, the subgroup of nonadvanced prostate cancers is predominated by less aggressive tumors that have low progression rates and may never reach a clinically relevant state. Distinct etiologies have been suggested and risk factor profiles of prostate tumors possibly vary depending on the stage at diagnosis [42-44]. MD adherence was not related to advanced prostate cancer risk in two US cohorts $[45,46]$.

In addition to the specific cancer types, overall cancer incidence is an interesting outcome to evaluate the potential of the MD as a dietary strategy to prevent cancer. So far, the cohort evidence on the association between MD adherence and overall cancer incidence has been inconclusive, with inverse relations being observed in some studies $[47,48]$, but not in others $[7,16,49]$. In this thesis, we showed that higher MD adherence was only associated with a non-significantly reduced cancer risk in women. In accordance with our findings, a stronger inverse association was observed in female participants of EPIC-Greece $\left(p_{\text {interaction }}>0.05\right)$ [47]. However, sex-specific associations did not vary in the remainder of the studies [7, 48, 49]. When investigating the effect of dietary factors on overall cancer incidence, one should realize that contributions of individual cancer types to the total cancer incidence vary depending on the study population. For example, a large proportion of the cancers diagnosed in men and women is made up by sex-specific tumors (e.g., tumors of the prostate and breast). As MD adherence seems to be related differently to specific cancer types, this could, at least partly, explain discrepancies in associations with overall cancer risk across the sexes or cohorts.

Integrating the findings of this thesis and other prospective studies, we may conclude that adherence to the MD possibly has a preventive effect on several cancer types, including cancers of the lung, postmenopausal breast, esophagus (ESCC in men), stomach, and colorectum (men only). It remains to be seen whether MD adherence is associated with a decreased risk of bladder cancer as well or that the observed inverse associations in the literature were the result of residual confounding by tobacco smoking. Heterogeneity of associations across the sexes and/or subtypes was indicated for cancers of the lung, breast, 
esophagus, (stomach), and colorectum. Unfortunately, the number of cohort studies reporting subtype-specific associations in men and women is still limited for many cancer sites. Potential mechanisms underlying the possible cancer-protective effect of the MD have already been discussed in the introduction of this thesis (Chapter 1 ).

\section{Methodological considerations}

\subsection{Moderate alcohol consumption and the Mediterranean diet}

The inclusion of moderate alcohol consumption as a favorable component in the MD is controversial. Traditionally, moderate amounts of red wine were consumed with meals in many Mediterranean regions $[50,51]$. Red wine is a source of antioxidant and antiinflammatory polyphenols, which may beneficially affect cardiovascular health [52]. It has been debated whether a light-to-moderate intake of red wine (and alcoholic beverages in general) exerts a cardioprotective effect [52]. Correspondingly, many MD scores consider a moderate alcohol intake beneficial. Alcohol consumption is generally not assumed to have a favorable effect on cancer risk at any level. Even at light or moderate intake levels, alcohol has been associated with elevated risks of cancers of the oral cavity and pharynx, larynx, esophagus (ESCC), colorectum, and female breast [2]. Therefore, in cancer prevention, a MD that does not allow any consumption of alcoholic drinks might be preferred. For this reason, we compared MD scores with and without an alcohol component, which has been done in other studies as well (e.g., $[15,22,31,38,41])$. For the majority of the cancer sites, an equal or better model fit was obtained for the MD score variant without alcohol (aMEDr). Furthermore, exclusion of the alcohol component did not seem to affect associations of MD adherence with cancer risk as was demonstrated by the essentially similar results for both score variants. Nevertheless, in our meta-analyses concerning postmenopausal breast cancer, higher MD adherence was only associated with a reduced risk when we combined results of studies that excluded alcohol from the MD score. Earlier cohort studies investigating breast cancer risk have reported virtually similar relations for MD scores with and without alcohol $[12,13,15,17]$. Based on the above, we recommend that alcohol is excluded from the MD score in future studies, especially when relations with cancer risk are investigated. As an alternative, MD scores with and without alcohol component could be compared.

\subsection{Olive oil and the Mediterranean diet}

The generous use of olive oil instead of other plant oils or animal fats characterizes the traditional MD $[50,51]$. This is reflected in the fatty acid profile of the MD that is high in monounsaturated fatty acids (MUFA, from olive oil), but low in saturated fatty acids (SFA) $[50,51,53]$. Olive oil consumption is not specifically scored in the traditional Mediterranean diet score (tMED, $[53,54])$ and many of its derivatives, including aMED $[55,56]$ and mMED [57]. Alternatively, the favorable fatty acid profile of the MD is taken into account through the inclusion of a fatty acid ratio (originally MUFA:SFA) in the score. By using the fatty acid ratio as a proxy for the high olive oil consumption in the MD, tMED and variants are also easy to apply in non-Mediterranean populations, where olive oil is typically less commonly used in the preparation of meals. At the time of the baseline measurements of the NLCS and EPIC-NL, the use of olive oil as the main dietary fat was rather uncommon in the Netherlands as well and olive oil consumption was therefore not specifically included as 
an item in the food frequency questionnaires (FFQs). In mMED [57], the fatty acid ratio was adapted to the dietary habits of non-Mediterranean populations by including both MUFA and polyunsaturated fatty acids (PUFA). The inclusion of PUFA in the fatty acid ratio was desired, because PUFA constitute the largest proportion of unsaturated fatty acids in these populations and are suggested to have beneficial health effects themselves. Additionally, meat (and not olive oil) is an important source of MUFA in non-Mediterranean countries.

In several analyses of the EPIC cohort (e.g., focusing on breast, pancreatic, and bladder cancer) similar associations were observed for MD scores that included the MUFA(+ PUFA):SFA ratio and scores that directly incorporated olive oil intake [10, 12, 36, 41]. This may indicate that the fatty acid ratio is a suitable way to model the high olive oil consumption that characterizes the MD.

\subsection{Dietary pattern analysis}

Human beings do not consume isolated foods and nutrients. Instead, dietary components are consumed in certain combinations and collectively compose an individual's dietary pattern. Synergistic or antagonistic interactions between dietary components may modify health effects of single foods or nutrients $[54,58,59]$. Therefore, in our attempt to gain insight into the possible cancer-protective effects of the MD and the potential of this diet in cancer prevention, dietary pattern analysis seemed to be the most fruitful approach. Besides acknowledging interactions between dietary components, dietary pattern analysis has some additional advantages. Firstly, the capability to reveal true health benefits of the MD is increased, because effects of singular components may be small and for that reason undetectable unless integrated into a pattern. Moreover, by considering the combined effect of all aspects of the $M D$, extremes of cumulative exposures are evaluated. Finally, collinearity and confounding issues arising from the high correlations between food groups are avoided [54, 58, 59]. Results of our analyses underlined the usefulness of the pattern-based approach. Although higher MD scores were associated with (non-significantly) reduced risks of cancers of the lung, breast (postmenopausal), esophagus (ESCC in men), and stomach (GCA and GNCA), associations for individual score components were sometimes absent or in unexpected directions. Additionally, inverse associations with these cancer types did not seem to be dominated by a single MD component.

\subsection{The use of cohort-specific cut-offs in the assessment of Mediterranean diet adherence}

\subsubsection{Dietary habits of the Dutch population in the 1990s}

A potential weakness of the MD scores that we applied in this thesis is the use of population medians as cut-offs to separate subjects with high and low intakes of the individual dietary components. Therefore, MD scores of individuals with identical dietary habits may vary depending on the diet of the remainder of the population. For example, a subject with a high MD score in the Dutch EPIC cohort may be classified as having an intermediate or low level of MD adherence in one of the southern European EPIC cohorts. The use of populationbased cut-offs in the assessment of MD adherence may particularly have consequences in non-Mediterranean populations where the overall level of MD adherence is generally low.

To get an idea about the extent to which the dietary habits of NLCS and EPIC-NL study participants resembled the traditional MD, we can compare mean daily intakes of typically (non-)Mediterranean food groups between the Dutch and Greek EPIC cohorts (EPIC-NL and 
EPIC-Greece, respectively). Between 1995 and 2000, single 24-hour dietary recalls were collected from random samples of the EPIC cohorts to estimate mean daily intakes of 22 food groups, adjusted for age, day of the week, and season [60]. Among men, mean daily intakes of vegetables, fruits (including nuts), and legumes were clearly lower in EPIC-NL, while the opposite was observed for meat intake (vegetables: 131 vs. $269 \mathrm{~g} /$ day; fruits: 156 vs. 234 g/day; legumes: 6 vs. 33 g/day; meat: 141 vs. 68 g/day). A similar pattern was reported when comparing female participants of the Dutch and Greek EPIC cohorts, with average daily intakes of 128 vs. $211 \mathrm{~g}$ for vegetables, $183 \mathrm{vs.} 218 \mathrm{~g}$ for fruits, $4 \mathrm{vs.} 21 \mathrm{~g}$ for legumes, and 80 vs. $35 \mathrm{~g}$ for meat, respectively.

Thus, appreciable differences existed between the dietary patterns of Dutch and Greek EPIC participants. The diet of the non-Mediterranean EPIC-NL cohort was characterized by a lower intake of plant foods and a higher intake of meat. Therefore, it seems likely that the diet of most individuals assigned to the highest MD adherence category in our analyses did not truly resemble a traditional MD. Nevertheless, these individuals may still experience lower cancer risks than subjects whose dietary habits deviated more from the MD as reflected by their lower MD scores. The MD is essentially an example of a plant-based dietary pattern, as there are several other index-based dietary patterns that are plant-based, such as the Healthy Eating Index-2015 (HEI-2015, [61]), the Alternative Healthy Eating Index-2010 (AHEI-2010, [62]), and the Dietary Approaches to Stop Hypertension (DASH, [63]) score, and does not necessarily have to be confined to the Mediterranean region.

\subsubsection{The absolute WCRF/AICR diet score for cancer prevention}

In this paragraph, we will focus on the likelihood that the population-based scoring in our assessment of MD adherence has affected our findings by comparing the relative aMED to absolute scores based on the dietary part of the cancer prevention guidelines developed by the World Cancer Research Fund/American Institute for Cancer Research (WCRF/AICR) in 2007 [64].

With the aim to prevent cancer, the WCRF/AICR published a list of recommendations in 2007 [64], of which six focused on diet and supplement use. In short, these dietary guidelines encouraged the consumption of plant foods, while stating that the intake of foods and drinks that promote weight gain, animal foods, alcoholic drinks, salt, and dietary supplements should be limited or avoided. In order to create an absolute WCRF/AICR diet score, we operationalized the recommendations concerning foods and drinks that promote weight gain, plant foods, red and processed meats, alcohol, and salt, using the same absolute cutoffs as earlier applied in the EPIC cohort $[65,66]$, whenever possible. Analogous to aMED, an additional score was made that did not incorporate the alcohol recommendation. For a detailed description of the calculation of the score, we refer to Chapter 3 of this thesis.

Focusing on scores that did not contain alcohol, we may conclude that the absolute WCRF/ AICR diet score did not outperform the cohort-specific aMEDr in our study population. Compared to aMEDr, model performances of the WCRF/AICR diet score were roughly similar (cancers of the lung, colorectum, and bladder) or worse (cancers of the breast and prostate). Additionally, associations per SD-increment in both scores pointed in the same direction for most of the investigated cancer sites. Unlike aMEDr, a SD-increase in the WCRF/AICR diet score was not inversely associated with breast cancer risk. 


\subsection{Strengths and limitations of the NLCS and EPIC-NL cohorts}

The prospective designs and long durations of follow-up are major strengths of the included cohorts that minimized the potential for information and selection biases. Furthermore, the large number of cancer diagnoses during follow-up for most cancer sites (particularly in the NLCS) allowed us to perform sex- and subtype-specific analyses with adequate statistical power and to evaluate the presence of effect modification by smoking status and other important cancer risk factors. However, case numbers for pancreatic cancer (MCPC) and subtypes of esophageal and gastric cancer were generally relatively small. Detailed dietary information of NLCS and EPIC-NL cohort members was retrieved via validated FFQs at baseline [67-69], but the possibility of errors in the dietary assessment cannot fully be excluded. A limitation of both cohorts is the absence of repeated dietary measurements during follow-up to update the baseline information. Therefore, we were not able to take possible changes in dietary habits or confounding factors after baseline into account in our analyses, which may have resulted in non-differential measurement error and attenuated associations. However, the reproducibility has been tested for both the NLCS and the EPIC-NL FFQ and was shown to be generally good [68-70]. Finally, residual confounding by unmeasured or imperfectly measured factors may have affected our results. Because we corrected our multivariable analyses for a large number of potential confounders, this influence is not likely to be strong.

Whenever possible, the abovementioned limitations should be addressed in future studies. We particularly encourage the repeated assessment of dietary habits at regular time intervals during follow-up.

\section{Implications for public health}

As we have already stated in Chapter 1 of this thesis, the cancer burden in the Netherlands is high [71-73]. A further increase is anticipated in the coming decades due to the ageing population and increasing life expectancy [74]. It has been estimated that about $10 \%$ of the incident cancer cases in the Dutch population in 2010 could have been prevented if everyone had adhered to a healthy diet [75]. According to this thesis, MD adherence may be associated with decreased risks of cancers of the lung, breast, esophagus (ESCC in men), and stomach in the Netherlands. Cancers of the lung, esophagus, and stomach have a relatively poor prognosis and are therefore particularly in need of successful preventive strategies. The literature has indicated an additional beneficial effect of MD adherence on the incidence of colorectal cancer (men only), as we have described earlier in this chapter.

So, is the current scientific evidence sufficient to promote the traditional MD specifically as a dietary strategy for cancer prevention? In our opinion, probably not.

Although the relation between MD adherence and cancer risk has been the topic of a considerable number of publications in the past few years, the cohort evidence is still limited, especially for some cancer (sub)sites. Additional prospective studies reporting sex-specific associations of MD adherence with incidence of cancer subsites may help to answer the remaining questions and to solve the inconsistencies. Randomized controlled trials (RCTs) that evaluate the effect of a MD intervention on cancer risk might be particularly helpful. As far as we know, no sizeable RCTs with this primary aim have been conducted yet. Our study adds to the currently available evidence, but does not suffice to enable the formulation of definitive conclusions concerning the cancer-protective effect of the MD in the Netherlands. 
Instead of promoting the traditional MD specifically with the aim to prevent cancer, policymakers in the Netherlands could use the MD as a basis to formulate a healthy plantbased dietary pattern for the prevention of chronic disease in general. Besides its potentially beneficial effect on cancer risk, higher MD adherence may be associated with reduced risks of several other chronic diseases (including CVD) and all-cause mortality (e.g., [76-80], see also Chapter 1 ), which enables the formulation of an unambiguous message to the Dutch population.

The MD is generally considered to be a delicious and versatile dietary pattern and MDstyle meals can be prepared rather quickly and easily if desired [81]. The tastiness of the $\mathrm{MD}$ as well as the absence of constraints to energy intake (though energy balance should be maintained) may improve the willingness of the Dutch people to adopt a more plantoriented diet. Murphy et al. [81] suggested that the versatility and palatability of the MD contributed to the high and long-term compliance that has been observed in MD intervention studies in Australia, which investigated effects of the MD on CVD risk factors and cognitive performance in elderly people and mental health in adults suffering from depression [8185].

Another advantage of the MD is its low impact on the environment compared to more Western dietary patterns [86-89]. The MD has been associated with a favorable water footprint and lower greenhouse gas emissions, because of its plant-based nature and the low consumption of animal foods [86-91]. After examining health effects, environmental footprints, and monetary costs of the Mediterranean, Provegetarian, and Western dietary patterns simultaneously, the MD appeared to be the most sustainable option [92].

In the previous paragraph, we discussed that the stimulation of a healthy plant-based dietary pattern, which incorporates some of the aspects of the traditional MD, may be an interesting approach in the prevention of chronic disease in the Netherlands. The following comments can be made regarding the constituents of this suggested MD-derived dietary pattern. In line with the MD, plant foods should play a central role in the promoted dietary pattern, which also encompasses the regular use of olive oil as the main culinary fat. Of note, the recommended dietary pattern does not need to be exclusively vegetarian and may allow the consumption of small and moderate amounts of meat and fish, respectively. Alcohol consumption, on the other hand, should be completely discouraged because of its carcinogenic effect in humans $[1,2]$. Perhaps, a suitable dietary pattern can be defined by adapting the MD to the local food availability as well as specific needs and preferences of the Dutch population [81]. Stimulation of the consumption of locally grown and seasonal foods, which also constitutes a core characteristic of the MD, may improve the sustainability of the dietary pattern even further $[93,94]$. Moreover, by allowing the consumption of, e.g., low-fat dairy products, the dietary pattern may better accommodate the preferences of the Dutch population, potentially improving the attitude of the general public to the proposed guidelines. With an average consumption of $352 \mathrm{~g}$ per day between 2012 and 2016, dairy products compose a substantial part of the present diet in the Netherlands [95]. For the suggested intake levels, we refer to the Dutch dietary guidelines 2015, which were issued by the Health Council of the Netherlands and were based on the latest scientific evidence concerning chronic disease risk [96]. The MD shows many similarities with these guidelines: both recommend an eating pattern that is abundant in plant foods, but limited in foods from animal origin. According to the Dutch dietary guidelines 2015, people should consume several servings of dairy each day, including milk or yoghurt.

Supportive measures, e.g., by policymakers and governmental institutions, are most likely required to successfully implement a healthy and more sustainable plant-based diet in the 
Dutch population [81]. The formulation of clear guidelines and the provision of recipes or meal plans could be very helpful to the public, facilitating an easier transition to a diet primarily based on plant foods [81]. Furthermore, efforts should be made to make plant foods accessible and affordable to all social classes. In this respect, one could think of guaranteeing the availability of a large variety of plant foods at local stores and reducing the tax on vegetables, fruits, and other foods of plant origin. Oppositely, the tax on meat and other unhealthy foods could be increased to make these foods more expensive and therefore less appealing to the general public.

With adequate governmental actions, clear guidelines, and support, the Dutch population should theoretically be able to change their present dietary behaviors for the better and to adopt a healthy plant-based dietary pattern. Besides promoting a healthy diet, it is of utmost importance in the prevention of cancer and other chronic diseases in the Netherlands to keep stimulating the public to quit smoking, to maintain a healthy body weight, and to be physically active. At the population level, adoption of a healthier lifestyle by the Dutch people might reduce the incidence of chronic diseases. However, it is challenging, if not impossible, to predict who will get ill at the individual level. Some men and women who adhere to a healthy lifestyle might still suffer from chronic disease in the future as a consequence of other exposures, genetics, or merely bad luck.

\section{Future recommendations}

The field of nutrition-related research is continuously evolving and future research on the MD could take a wide array of directions. In both this chapter and previous chapters of this thesis, we have already briefly pinpointed some areas in which additional research is warranted. In this section, we would like to highlight some of our recommendations for future research.

Firstly, the relation between MD adherence and cancer risk has primarily been investigated in studies with observational designs. However, evidence for causal relationships can only be obtained from well-designed RCTs [97]. To our knowledge, no well-designed, adequatelysized RCTs with a long duration of follow-up have been conducted thus far with the principal aim to evaluate the effect of a MD-like dietary intervention on cancer risk. Such studies are desired, in both Mediterranean and non-Mediterranean countries, and may enable the formulation of definitive conclusions concerning the potentially cancer-protective effect of the MD.

A disadvantage of large and long-term dietary intervention trials is that they are costly, labor-intensive, and not always feasible [97-99]. Provided that the obtained results are consistent, large and well-designed cohort studies may still produce convincing evidence that suffices for the establishment of dietary guidelines [98-100]. In order to add to the currently available evidence, future cohort studies evaluating the relation of MD adherence with cancer risk should meet at least one of the following requirements:

- Subtype-specific associations between MD adherence and cancer incidence are evaluated separately for men and women.

- Improved methods are utilized for the measurement of dietary intake. These could be based on new technologies, such as apps or barcode scanners. Preferably, dietary information is updated at regular time intervals during follow-up.

- The absolute level of MD adherence is assessed using an evidence-based score. Up until now, the large majority of the studies measured the relative level of MD 
adherence using tMED or one of its many derivatives. An example of an evidencebased score that assesses the absolute level of MD adherence is the literaturebased adherence score to the Mediterranean diet (MEDI-LITE) developed by Sofi et al. [77, 101]. In order to define absolute cut-off values for the included food groups, median (or mean) consumption levels were retrieved from all cohort studies evaluating the health effects of the MD and subsequently combined.

Thus far, a large variety of a priori defined MD indexes has been created to assess MD adherence. These MD indexes were reported to differ in respect to multiple facets, including the number and types (e.g., food groups, nutrients etc.) of components, the scoring method, and, if applicable, the specific dietary components incorporated [102]. Moreover, disparities were noted in the definition and/or assessment of the dietary components [102]. These differences may have contributed to the generally rather weak correlations between $a$ priori defined MD indexes [103]. Therefore, homogeneity regarding the dietary components included in the MD indexes and the scoring criteria used has been advocated [103].

Besides the MD, several other a priori index-based dietary patterns exist, like the HEI2015 [61], the AHEI-2010 [62], and the DASH score [63]. All these index-based dietary patterns (including the MD) have in common that their scores increase with a more plantoriented diet. Various studies have compared associations of multiple index-based dietary patterns (including the MD) with mortality in a prospective setting, among others as part of the Dietary Patterns Methods Project [104-107]. However, comparable studies for risk of cancer in general or the majority of the individual cancer types have been performed less frequently, while these could be very useful in the identification of the most effective plantbased dietary pattern(s) for cancer prevention. Therefore, we recommend that such studies will be conducted in the future. Research aiming to unravel the underlying mechanisms may also be helpful in this regard. 


\section{References}

1. International Agency for Research on Cancer. Vol. 100E: a review of human carcinogens: personal habits and indoor combustions, IARC monographs on the evaluation of carcinogenic risks to humans. Lyon, France: IARC; 2012.

2. Bagnardi V, Rota M, Botteri E, Tramacere I, Islami F, Fedirko V, et al. Alcohol consumption and site-specific cancer risk: a comprehensive dose-response meta-analysis. Br J Cancer. 2015;112(3):58093.

3. World Cancer Research Fund / American Institute for Cancer Research. Diet, nutrition, physical activity and cancer: a global perspective. Continuous update project expert report 2018. 2018. Available from: https://www.wcrf.org/dietandcancer.

4. Gnagnarella P, Maisonneuve P, Bellomi M, Rampinelli C, Bertolotti R, Spaggiari L, et al. Red meat, Mediterranean diet and lung cancer risk among heavy smokers in the COSMOS screening study. Ann Oncol. 2013;24(10):2606-11.

5. Anic GM, Park Y, Subar AF, Schap TE, Reedy J. Index-based dietary patterns and risk of lung cancer in the NIH-AARP diet and health study. Eur J Clin Nutr. 2016;70(1):123-9.

6. Hodge AM, Bassett JK, Shivappa N, Hebert JR, English DR, Giles GG, et al. Dietary inflammatory index, Mediterranean diet score, and lung cancer: a prospective study. Cancer Causes Control. 2016;27(7):907-17.

7. Boden S, Myte R, Wennberg M, Harlid S, Johansson I, Shivappa N, et al. The inflammatory potential of diet in determining cancer risk; A prospective investigation of two dietary pattern scores. PLoS One. 2019;14(4):e0214551.

8. Toledo E, Salas-Salvado J, Donat-Vargas C, Buil-Cosiales P, Estruch R, Ros E, et al. Mediterranean diet and invasive breast cancer risk among women at high cardiovascular risk in the PREDIMED trial: A randomized clinical trial. JAMA Intern Med. 2015;175(11):1752-60.

9. Fung TT, Hu FB, McCullough ML, Newby PK, Willett WC, Holmes MD. Diet quality is associated with the risk of estrogen receptor-negative breast cancer in postmenopausal women. J Nutr. 2006;136(2):466-72.

10. Trichopoulou A, Bamia C, Lagiou P, Trichopoulos D. Conformity to traditional Mediterranean diet and breast cancer risk in the Greek EPIC (European Prospective Investigation into Cancer and Nutrition) cohort. Am J Clin Nutr. 2010;92(3):620-5.

11. Cade JE, Taylor EF, Burley VJ, Greenwood DC. Does the Mediterranean dietary pattern or the Healthy Diet Index influence the risk of breast cancer in a large British cohort of women? Eur J Clin Nutr. 2011;65(8):920-8.

12. Buckland G, Travier N, Cottet V, Gonzalez CA, Lujan-Barroso L, Agudo A, et al. Adherence to the Mediterranean diet and risk of breast cancer in the European Prospective Investigation into Cancer and Nutrition cohort study. Int J Cancer. 2013;132(12):2918-27.

13. Couto E, Sandin S, Lof M, Ursin G, Adami HO, Weiderpass E. Mediterranean dietary pattern and risk of breast cancer. PLoS One. 2013;8(2):e55374.

14. Hirko KA, Willett WC, Hankinson SE, Rosner BA, Beck AH, Tamimi RM, et al. Healthy dietary patterns and risk of breast cancer by molecular subtype. Breast Cancer Res Treat. 2016;155(3):579-88. 15. Haridass V, Ziogas A, Neuhausen SL, Anton-Culver H, Odegaard AO. Diet Quality Scores Inversely Associated with Postmenopausal Breast Cancer Risk Are Not Associated with Premenopausal Breast Cancer Risk in the California Teachers Study. J Nutr. 2018;148(11):1830-7.

16. Lavalette C, Adjibade M, Srour B, Sellem L, Fiolet T, Hercberg S, et al. Cancer-Specific and General Nutritional Scores and Cancer Risk: Results from the Prospective NutriNet-Sante Cohort. Cancer Res. 2018;78(15):4427-35.

17. Petimar J, Park YM, Smith-Warner SA, Fung TT, Sandler DP. Dietary index scores and invasive breast cancer risk among women with a family history of breast cancer. Am J Clin Nutr. 2019;109(5):1393-401.

18. Loman N, Johannsson O, Kristoffersson U, Olsson H, Borg A. Family history of breast and ovarian cancers and BRCA1 and BRCA2 mutations in a population-based series of early-onset breast cancer. J Natl Cancer Inst. 2001;93(16):1215-23.

19. Lagiou P, Hsieh CC, Trichopoulos D, Adami HO, Hall P, Chie L, et al. Neonatal growth and breast cancer risk in adulthood. Br J Cancer. 2008;99(9):1544-8. 
20. Kamangar F, Chow WH, Abnet CC, Dawsey SM. Environmental causes of esophageal cancer. Gastroenterol Clin North Am. 2009;38(1):27-57, vii.

21. Domper Arnal MJ, Ferrandez Arenas A, Lanas Arbeloa A. Esophageal cancer: Risk factors, screening and endoscopic treatment in Western and Eastern countries. World J Gastroenterol. 2015;21(26):7933-43.

22. Li WQ, Park Y, Wu JW, Ren JS, Goldstein AM, Taylor PR, et al. Index-based dietary patterns and risk of esophageal and gastric cancer in a large cohort study. Clin Gastroenterol Hepatol. 2013;11(9):1130-6 e2.

23. Buckland G, Agudo A, Lujan L, Jakszyn P, Bueno-de-Mesquita HB, Palli D, et al. Adherence to a Mediterranean diet and risk of gastric adenocarcinoma within the European Prospective Investigation into Cancer and Nutrition (EPIC) cohort study. Am J Clin Nutr. 2010;91(2):381-90.

24. Riboli E, Hunt KJ, Slimani N, Ferrari P, Norat T, Fahey M, et al. European Prospective Investigation into Cancer and Nutrition (EPIC): study populations and data collection. Public Health Nutr. 2002;5(6B):1113-24.

25. Crew KD, Neugut Al. Epidemiology of gastric cancer. World J Gastroenterol. 2006;12(3):354-

62.

26. Reedy J, Mitrou PN, Krebs-Smith SM, Wirfalt E, Flood A, Kipnis V, et al. Index-based dietary patterns and risk of colorectal cancer: the NIH-AARP Diet and Health Study. Am J Epidemiol. 2008;168(1):38-48.

27. Agnoli C, Grioni S, Sieri S, Palli D, Masala G, Sacerdote C, et al. Italian Mediterranean Index and risk of colorectal cancer in the Italian section of the EPIC cohort. Int J Cancer. 2013;132(6):140411.

28. Bamia C, Lagiou P, Buckland G, Grioni S, Agnoli C, Taylor AJ, et al. Mediterranean diet and colorectal cancer risk: results from a European cohort. Eur J Epidemiol. 2013;28(4):317-28.

29. Park SY, Boushey CJ, Wilkens LR, Haiman CA, Le Marchand L. High-Quality Diets Associate With Reduced Risk of Colorectal Cancer: Analyses of Diet Quality Indexes in the Multiethnic Cohort. Gastroenterology. 2017;153(2):386-94.

30. Stone RAT, Waring ME, Cutrona SL, Kiefe Cl, Allison J, Doubeni CA. The association of dietary quality with colorectal cancer among normal weight, overweight and obese men and women: a prospective longitudinal study in the USA. Bmj Open. 2017;7(6).

31. Petimar J, Smith-Warner SA, Fung TT, Rosner B, Chan AT, Hu FB, et al. Recommendationbased dietary indexes and risk of colorectal cancer in the Nurses' Health Study and Health Professionals Follow-up Study. Am J Clin Nutr. 2018;108(5):1092-103.

32. Vargas AJ, Neuhouser ML, George SM, Thomson CA, Ho GY, Rohan TE, et al. Diet Quality and Colorectal Cancer Risk in the Women's Health Initiative Observational Study. Am J Epidemiol. 2016;184(1):23-32.

33. Cheng E, Um CY, Prizment AE, Lazovich D, Bostick RM. Evolutionary-Concordance Lifestyle and Diet and Mediterranean Diet Pattern Scores and Risk of Incident Colorectal Cancer in Iowa Women. Cancer Epidemiol Biomarkers Prev. 2018;27(10):1195-202.

34. Lee GH, Malietzis G, Askari A, Bernardo D, Al-Hassi HO, Clark SK. Is right-sided colon cancer different to left-sided colorectal cancer? - a systematic review. Eur J Surg Oncol. 2015;41(3):300-8.

35. Wei EK, Giovannucci E, Wu K, Rosner B, Fuchs CS, Willett WC, et al. Comparison of risk factors for colon and rectal cancer. Int J Cancer. 2004;108(3):433-42.

36. Buckland G, Ros MM, Roswall N, Bueno-de-Mesquita HB, Travier N, Tjonneland A, et al. Adherence to the Mediterranean diet and risk of bladder cancer in the EPIC cohort study. Int J Cancer. 2014;134(10):2504-11.

37. Dugue PA, Hodge AM, Brinkman MT, Bassett JK, Shivappa N, Hebert JR, et al. Association between selected dietary scores and the risk of urothelial cell carcinoma: A prospective cohort study. Int J Cancer. 2016;139(6):1251-60.

38. Witlox WJA, van Osch FHM, Brinkman M, Jochems S, Goossens ME, Weiderpass E, et al. An inverse association between the Mediterranean diet and bladder cancer risk: a pooled analysis of 13 cohort studies. Eur J Nutr. 2019.

39. Cumberbatch MGK, Jubber I, Black PC, Esperto F, Figueroa JD, Kamat AM, et al. Epidemiology of Bladder Cancer: A Systematic Review and Contemporary Update of Risk Factors in 2018. Eur Urol. 2018;74(6):784-95. 


\section{Chapter 9}

40. Jiao L, Mitrou PN, Reedy J, Graubard BI, Hollenbeck AR, Schatzkin A, et al. A combined healthy lifestyle score and risk of pancreatic cancer in a large cohort study. Arch Intern Med. 2009;169(8):76470 .

41. Molina-Montes E, Sanchez MJ, Buckland G, Bueno-de-Mesquita HB, Weiderpass E, Amiano $P$, et al. Mediterranean diet and risk of pancreatic cancer in the European Prospective Investigation into Cancer and Nutrition cohort. Br J Cancer. 2017;116(6):811-20.

42. Hsing AW, Chokkalingam AP. Prostate cancer epidemiology. Front Biosci. 2006;11:1388-413.

43. Giovannucci E, Liu Y, Platz EA, Stampfer MJ, Willett WC. Risk factors for prostate cancer incidence and progression in the health professionals follow-up study. Int J Cancer. 2007;121(7):15718.

44. Jahn JL, Giovannucci EL, Stampfer MJ. The high prevalence of undiagnosed prostate cancer at autopsy: implications for epidemiology and treatment of prostate cancer in the Prostate-specific Antigen-era. Int J Cancer. 2015;137(12):2795-802.

45. Bosire C, Stampfer MJ, Subar AF, Park Y, Kirkpatrick SI, Chiuve SE, et al. Index-based dietary patterns and the risk of prostate cancer in the NIH-AARP diet and health study. Am J Epidemiol. 2013;177(6):504-13.

46. Kenfield SA, DuPre N, Richman EL, Stampfer MJ, Chan JM, Giovannucci EL. Mediterranean diet and prostate cancer risk and mortality in the Health Professionals Follow-up Study. Eur Urol. 2014;65(5):887-94.

47. Benetou V, Trichopoulou A, Orfanos P, Naska A, Lagiou P, Boffetta P, et al. Conformity to traditional Mediterranean diet and cancer incidence: the Greek EPIC cohort. $\mathrm{Br} \mathrm{J}$ Cancer. 2008;99(1):191-5.

48. Couto E, Boffetta P, Lagiou P, Ferrari P, Buckland G, Overvad K, et al. Mediterranean dietary pattern and cancer risk in the EPIC cohort. Br J Cancer. 2011;104(9):1493-9.

49. Galbete C, Kroger J, Jannasch F, Iqbal K, Schwingshackl L, Schwedhelm C, et al. Nordic diet, Mediterranean diet, and the risk of chronic diseases: the EPIC-Potsdam study. BMC Med. 2018;16(1):99.

50. Willett WC, Sacks F, Trichopoulou A, Drescher G, Ferro-Luzzi A, Helsing E, et al. Mediterranean diet pyramid: a cultural model for healthy eating. Am J Clin Nutr. 1995;61(6 Suppl):1402S-6S.

51. Trichopoulou A, Lagiou P. Healthy traditional Mediterranean diet: an expression of culture, history, and lifestyle. Nutr Rev. 1997;55(11 Pt 1):383-9.

52. Haseeb S, Alexander B, Baranchuk A. Wine and Cardiovascular Health: A Comprehensive Review. Circulation. 2017;136(15):1434-48.

53. Trichopoulou A, Kouris-Blazos A, Wahlqvist ML, Gnardellis C, Lagiou P, Polychronopoulos E, et al. Diet and overall survival in elderly people. BMJ. 1995;311(7018):1457-60.

54. Trichopoulou A, Costacou T, Bamia C, Trichopoulos D. Adherence to a Mediterranean diet and survival in a Greek population. N Engl J Med. 2003;348(26):2599-608.

55. Fung TT, McCullough ML, Newby PK, Manson JE, Meigs JB, Rifai N, et al. Diet-quality scores and plasma concentrations of markers of inflammation and endothelial dysfunction. Am J Clin Nutr. 2005;82(1):163-73.

56. Mitrou PN, Kipnis V, Thiebaut AC, Reedy J, Subar AF, Wirfalt E, et al. Mediterranean dietary pattern and prediction of all-cause mortality in a US population: results from the NIH-AARP Diet and Health Study. Arch Intern Med. 2007;167(22):2461-8.

57. Trichopoulou A, Orfanos P, Norat T, Bueno-de-Mesquita B, Ocke MC, Peeters PH, et al. Modified Mediterranean diet and survival: EPIC-elderly prospective cohort study. BMJ. 2005;330(7498):991.

58. Jacques PF, Tucker KL. Are dietary patterns useful for understanding the role of diet in chronic disease? Am J Clin Nutr. 2001;73(1):1-2.

59. Verberne L, Bach-Faig A, Buckland G, Serra-Majem L. Association between the Mediterranean diet and cancer risk: a review of observational studies. Nutr Cancer. 2010;62(7):860-70.

60. Slimani N, Fahey M, Welch AA, Wirfalt E, Stripp C, Bergstrom E, et al. Diversity of dietary patterns observed in the European Prospective Investigation into Cancer and Nutrition (EPIC) project. Public Health Nutr. 2002;5(6B):1311-28.

61. Krebs-Smith SM, Pannucci TE, Subar AF, Kirkpatrick SI, Lerman JL, Tooze JA, et al. Update of the Healthy Eating Index: HEI-2015. J Acad Nutr Diet. 2018;118(9):1591-602.

62. Chiuve SE, Fung TT, Rimm EB, Hu FB, McCullough ML, Wang M, et al. Alternative dietary 
indices both strongly predict risk of chronic disease. J Nutr. 2012;142(6):1009-18.

63. Fung TT, Chiuve SE, McCullough ML, Rexrode KM, Logroscino G, Hu FB. Adherence to a DASHstyle diet and risk of coronary heart disease and stroke in women. Arch Intern Med. 2008;168(7):71320.

64. World Cancer Research Fund / American Institute for Cancer Research. Food, nutrition, physical activity, and the prevention of cancer: a global perspective. Washington DC: American Institute for Cancer Research; 2007.

65. Romaguera D, Vergnaud AC, Peeters PH, van Gils CH, Chan DS, Ferrari P, et al. Is concordance with World Cancer Research Fund/American Institute for Cancer Research guidelines for cancer prevention related to subsequent risk of cancer? Results from the EPIC study. Am J Clin Nutr. 2012;96(1):150-63.

66. Vergnaud AC, Romaguera D, Peeters PH, van Gils CH, Chan DS, Romieu I, et al. Adherence to the World Cancer Research Fund/American Institute for Cancer Research guidelines and risk of death in Europe: results from the European Prospective Investigation into Nutrition and Cancer cohort study. Am J Clin Nutr. 2013;97(5):1107-20.

67. Goldbohm RA, van den Brandt PA, Brants HA, van 't Veer P, Al M, Sturmans F, et al. Validation of a dietary questionnaire used in a large-scale prospective cohort study on diet and cancer. Eur J Clin Nutr. 1994;48(4):253-65.

68. Ocke MC, Bueno-de-Mesquita HB, Goddijn HE, Jansen A, Pols MA, van Staveren WA, et al. The Dutch EPIC food frequency questionnaire. I. Description of the questionnaire, and relative validity and reproducibility for food groups. Int J Epidemiol. 1997;26 Suppl 1:S37-48.

69. Ocke MC, Bueno-de-Mesquita HB, Pols MA, Smit HA, van Staveren WA, Kromhout D. The Dutch EPIC food frequency questionnaire. II. Relative validity and reproducibility for nutrients. Int J Epidemiol. 1997;26 Suppl 1:S49-58.

70. Goldbohm RA, van 't Veer P, van den Brandt PA, van 't Hof MA, Brants HA, Sturmans F, et al. Reproducibility of a food frequency questionnaire and stability of dietary habits determined from five annually repeated measurements. Eur J Clin Nutr. 1995;49(6):420-9.

71. Bray F, Ferlay J, Soerjomataram I, Siegel RL, Torre LA, Jemal A. Global cancer statistics 2018: GLOBOCAN estimates of incidence and mortality worldwide for 36 cancers in 185 countries. CA Cancer J Clin. 2018;68(6):394-424.

72. Ferlay J, Ervik M, Lam F, Colombet M, Mery L, Pineros M, et al. Global Cancer Observatory: Cancer Today. Lyon, France: International Agency for Research on Cancer; 2018 [cited 2019 November]. Available from: https://gco.iarc.fr/today.

73. Ferlay J, Colombet M, Soerjomataram I, Mathers C, Parkin DM, Pineros M, et al. Estimating the global cancer incidence and mortality in 2018: GLOBOCAN sources and methods. Int J Cancer. 2019;144(8):1941-53.

74. Siesling S, Visser O, Aarts MJ, Verhoeven RHA, Aben KKH, Dinmohamed AG, et al. [Fight against cancer in the Netherlands: current state of affairs]. Ned Tijdschr Geneeskd. 2019;163 (in Dutch).

75. Lanting $\mathrm{Cl}$, de Vroome EM, Elias SG, van den Brandt PA, van Leeuwen FE, Kampman E, et al. [Contribution of lifestyle factors to cancer: secondary analysis of Dutch data over 2010 and a projection for 2020]. Ned Tijdschr Geneeskd. 2014;159:A8085 (in Dutch).

76. Martinez-Gonzalez MA, Bes-Rastrollo M. Dietary patterns, Mediterranean diet, and cardiovascular disease. Curr Opin Lipidol. 2014;25(1):20-6.

77. Sofi F, Macchi C, Abbate R, Gensini GF, Casini A. Mediterranean diet and health status: an updated meta-analysis and a proposal for a literature-based adherence score. Public Health Nutr. 2014;17(12):2769-82.

78. Grosso G, Marventano S, Yang J, Micek A, Pajak A, Scalfi L, et al. A comprehensive metaanalysis on evidence of Mediterranean diet and cardiovascular disease: Are individual components equal? Crit Rev Food Sci Nutr. 2017;57(15):3218-32.

79. Dinu M, Pagliai G, Casini A, Sofi F. Mediterranean diet and multiple health outcomes: an umbrella review of meta-analyses of observational studies and randomised trials. Eur J Clin Nutr. 2018;72(1):30-43.

80. Martinez-Lacoba R, Pardo-Garcia I, Amo-Saus E, Escribano-Sotos F. Mediterranean diet and health outcomes: a systematic meta-review. Eur J Public Health. 2018;28(5):955-61. 
81. Murphy KJ, Parletta N. Implementing a Mediterranean-Style Diet Outside the Mediterranean Region. Curr Atheroscler Rep. 2018;20(6):28.

82. Knight A, Bryan J, Wilson C, Hodgson J, Murphy K. A randomised controlled intervention trial evaluating the efficacy of a Mediterranean dietary pattern on cognitive function and psychological wellbeing in healthy older adults: the MedLey study. BMC Geriatr. 2015;15:55.

83. Jacka FN, O'Neil A, Opie R, Itsiopoulos C, Cotton S, Mohebbi M, et al. A randomised controlled trial of dietary improvement for adults with major depression (the 'SMILES' trial). BMC Med. 2017;15(1):23.

84. Opie RS, O'Neil A, Jacka FN, Pizzinga J, Itsiopoulos C. A modified Mediterranean dietary intervention for adults with major depression: Dietary protocol and feasibility data from the SMILES trial. Nutr Neurosci. 2018;21(7):487-501.

85. Parletta N, Zarnowiecki D, Cho J, Wilson A, Bogomolova S, Villani A, et al. A Mediterraneanstyle dietary intervention supplemented with fish oil improves diet quality and mental health in people with depression: A randomized controlled trial (HELFIMED). Nutr Neurosci. 2019;22(7):474-87.

86. Tukker A, Goldbohm RA, de Koning A, Verheijden M, Kleijn R, Wolf O, et al. Environmental impacts of changes to healthier diets in Europe. Ecol Econ. 2011;70(10):1776-88.

87. van Dooren C, Marinussen M, Blonk H, Aiking H, Vellinga P. Exploring dietary guidelines based on ecological and nutritional values: A comparison of six dietary patterns. Food Policy. 2014;44:36-46.

88. Tilman D, Clark M. Global diets link environmental sustainability and human health. Nature. 2014;515(7528):518-22.

89. Dernini S, Berry EM, Serra-Majem L, La Vecchia C, Capone R, Medina FX, et al. Med Diet 4.0: the Mediterranean diet with four sustainable benefits. Public Health Nutr. 2017;20(7):1322-30.

90. Heller MC, Keoleian GA, Willett WC. Toward a life cycle-based, diet-level framework for food environmental impact and nutritional quality assessment: a critical review. Environ Sci Technol. 2013;47(22):12632-47.

91. Vanham D, Mekonnen MM, Hoekstra AY. The water footprint of the EU for different diets. Ecol Indic. 2013;32:1-8.

92. Fresan U, Martinez-Gonzalez MA, Sabate J, Bes-Rastrollo M. Global sustainability (health, environment and monetary costs) of three dietary patterns: results from a Spanish cohort (the SUN project). BMJ Open. 2019;9(2):e021541.

93. Martinez-Gonzalez MA, Hershey MS, Zazpe I, Trichopoulou A. Transferability of the Mediterranean Diet to Non-Mediterranean Countries. What Is and What Is Not the Mediterranean Diet. Nutrients. 2017;9(11).

94. Martinez-Gonzalez MA, Hershey MS, Zazpe I, Trichopoulou A. Correction: MartinezGonzalez, M.A. et al. Transferability of the Mediterranean Diet to Non-Mediterranean Countries. What Is and What Is Not the Mediterranean Diet. Nutrients 2017, 9, 1226. Nutrients. 2018;10(7).

95. National Institute for Public Health and the Environment. Dutch National Food Consumption Survey 2012-2016. Bilthoven, the Netherlands: National Institute for Public Health and the Environment; 2018 [cited 2019 November]. Available from: https://www.wateetnederland.nl/ (in Dutch).

96. Health Council of the Netherlands. Richtlijn goede voeding 2015. The Hague, the Netherlands: Health Council of the Netherlands; 2015; publication number: 2015/24 (in Dutch).

97. Laville M, Segrestin B, Alligier M, Ruano-Rodriguez C, Serra-Majem L, Hiesmayr M, et al. Evidence-based practice within nutrition: what are the barriers for improving the evidence and how can they be dealt with? Trials. 2017;18(1):425.

98. Blumberg J, Heaney RP, Huncharek M, Scholl T, Stampfer M, Vieth R, et al. Evidence-based criteria in the nutritional context. Nutr Rev. 2010;68(8):478-84.

99. Satija A, Yu E, Willett WC, Hu FB. Understanding nutritional epidemiology and its role in policy. Adv Nutr. 2015;6(1):5-18.

100. Serra-Majem L, Roman-Vinas B, Sanchez-Villegas A, Guasch-Ferre M, Corella D, La Vecchia C. Benefits of the Mediterranean diet: Epidemiological and molecular aspects. Mol Aspects Med. 2019;67:1-55.

101. Sofi F, Dinu M, Pagliai G, Marcucci R, Casini A. Validation of a literature-based adherence score to Mediterranean diet: the MEDI-LITE score. Int J Food Sci Nutr. 2017;68(6):757-62.

102. Hernandez-Ruiz A, Garcia-Villanova B, Guerra Hernandez EJ, Amiano P, Azpiri M, Molina- 
Montes E. Description of indexes based on the adherence to the Mediterranean Dietary Pattern: a review. Nutr Hosp. 2015;32(5):1872-84.

103. Mila-Villarroel R, Bach-Faig A, Puig J, Puchal A, Farran A, Serra-Majem L, et al. Comparison and evaluation of the reliability of indexes of adherence to the Mediterranean diet. Public Health Nutr. 2011;14(12A):2338-45.

104. George SM, Ballard-Barbash R, Manson JE, Reedy J, Shikany JM, Subar AF, et al. Comparing indices of diet quality with chronic disease mortality risk in postmenopausal women in the Women's Health Initiative Observational Study: evidence to inform national dietary guidance. Am J Epidemiol. 2014;180(6):616-25.

105. Reedy J, Krebs-Smith SM, Miller PE, Liese AD, Kahle LL, Park Y, et al. Higher diet quality is associated with decreased risk of all-cause, cardiovascular disease, and cancer mortality among older adults. J Nutr. 2014;144(6):881-9.

106. Harmon BE, Boushey CJ, Shvetsov YB, Ettienne R, Reedy J, Wilkens LR, et al. Associations of key diet-quality indexes with mortality in the Multiethnic Cohort: the Dietary Patterns Methods Project. Am J Clin Nutr. 2015;101(3):587-97.

107. Liese AD, Krebs-Smith SM, Subar AF, George SM, Harmon BE, Neuhouser ML, et al. The Dietary Patterns Methods Project: synthesis of findings across cohorts and relevance to dietary guidance. J Nutr. 2015;145(3):393-402. 


\title{
Addendum
}

\author{
Summary
}

Samenvatting

Valorization

Dankwoord

Curriculum Vitae

List of publications

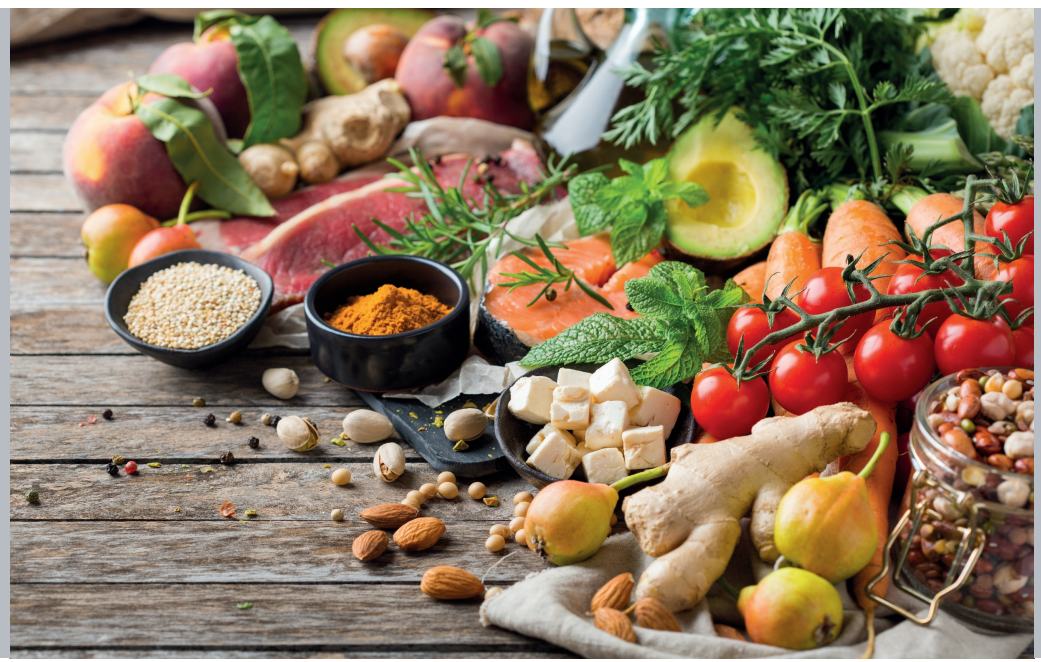




\section{Summary}

With its high incidence and mortality, cancer negatively affects many lives. According to estimations, about a tenth of the cancers diagnosed in the Netherlands in 2010 would have been preventable by the adoption of a healthy diet. The traditional Mediterranean diet (MD) can be defined as the dietary pattern typical of the olive-cultivating areas bordering the Mediterranean Sea in the late 1950s and the early 1960s, which was primarily based on plant foods (Chapter 1). The MD was characterized by a high consumption of vegetables, fruits, legumes, nuts, whole grains, and olive oil (rich in monounsaturated fatty acids). In contrast, the intake of meat and dairy products was low. Alcohol was consumed in moderate amounts and usually during meals. Adherence to the MD has been associated with numerous health benefits, including reduced all-cause mortality, and cardiovascular disease incidence and mortality. Despite the increasing interest in the potential cancer-protective effect of MD adherence in recent years, the evidence is still limited for most cancer sites. Additionally, results of conducted studies were not always consistent and had often been obtained using case-control designs, which are prone to bias. Moreover, potential heterogeneity of associations with MD adherence across the sexes or subtypes of specific cancer sites has been suggested, but has rarely been evaluated in prospective studies. Therefore, the principal aim of this thesis was to prospectively evaluate the association of MD adherence with incidence of specific cancer sites (i.e., lung, breast, esophagus, stomach, pancreas, colorectum, prostate, and bladder) as well as overall cancer incidence in the Netherlands. Specific attention was paid to possible differences in associations between men and women, and across subtypes of the investigated cancer sites. The relative level of MD adherence was assessed using two a priori defined MD scores, namely the alternate Mediterranean diet score (aMED) and the modified Mediterranean diet score (mMED). Alcohol consumption may increase the risk of multiple types of cancer even at low or moderate intake levels. Therefore, reduced variants of aMED and MMED were created that did not include the alcohol component (aMEDr and mMEDr, respectively) and models containing MD score variants with and without alcohol were compared in terms of performance. In order to investigate our aims, we primarily used data collected from the 120852 participants of the population-based Netherlands Cohort Study on Diet and Cancer (NLCS), who were between the ages of 55 and 69 years at baseline in September 1986. To increase statistical power, the association between MD adherence and pancreatic cancer risk was evaluated by pooling results from the NLCS and the Dutch cohort of the European Prospective Investigation into Cancer and Nutrition (EPIC-NL). The EPIC-NL cohort includes 40011 men and women, who were aged 20 to 70 years at enrolment between 1993 and 1997. NLCS participants were followed up for cancer incidence for a maximum of 20.3 years, whereas the median followup in EPIC-NL was 19.2 years.

For the majority of the cancer sites, aMEDr-containing models had an equal or better performance than mMEDr-containing models. Therefore, aMEDr was considered our principal measure of MD adherence. Furthermore, the score variant without alcohol component was preferred, because of the carcinogenic effect of alcohol in humans.

Higher MD adherence (aMEDr) was associated with a non-significantly reduced lung cancer risk in men and women (Chapter 2). Subgroup analyses suggested that the non-significant inverse association was most pronounced in women and those who never smoked, but the interaction tests did not reach statistical significance. When comparing associations with aMEDr across the histological lung cancer subtypes, some variations in strength were observed, especially in men.

Increasing levels of MD adherence were also associated with a non-significantly reduced 
incidence of postmenopausal breast cancer in female NLCS participants (Chapter 3). Stratification by estrogen receptor (ER) status showed that the inverse association was strongest and only statistically significant for the ER negative subtype. Similar findings were obtained when we evaluated progesterone receptor (PR) and combined ER/PR subtypes. Finally, we combined our results of the NLCS and results of previously published cohort studies in random-effects meta-analyses. Results of these meta-analyses were in line with the NLCS observations, showing inverse associations between MD adherence and postmenopausal breast cancer risk, particularly of the ER negative subtype.

For esophageal and gastric cancer (Chapter 4), associations with MD adherence were evaluated separately for subtypes defined by histology and anatomic location, respectively, which were suggested to have distinct etiological backgrounds. A significantly decreased risk of esophageal squamous cell carcinoma (ESCC) was observed among men with higher levels of MD adherence. In contrast, MD adherence was not associated with ESCC risk in women or risk of esophageal adenocarcinoma (EAC) in both men and women. Although statistical significance was only reached in men, MD adherence was inversely associated with risks of gastric cardia adenocarcinoma (GCA) and gastric non-cardia adenocarcinoma (GNCA) in both sexes.

Using data of both the NLCS and EPIC-NL cohorts, the relation of MD adherence with pancreatic cancer incidence was investigated in Chapter 5. MD adherence was not significantly associated with the risk of microscopically confirmed pancreatic cancer (MCPC) in pooled and cohort-specific analyses, irrespective of sex. Potential effect modification by smoking status was indicated. MD adherence was weakly and non-significantly inversely associated with MCPC risk in never smokers, but not in ever smokers. The overall conclusion did not change when we also included cases who were not microscopically confirmed in the analyses.

Furthermore, MD adherence was not significantly associated with colorectal cancer risk in men and women, regardless of the anatomical subsite (i.e., colon, proximal colon, distal colon, and rectum) evaluated (Chapter 6$)$.

Associations of MD adherence with risks of prostate and bladder cancer were examined in Chapter 7. For prostate cancer, associations were estimated separately for advanced and nonadvanced tumors at diagnosis, because differences in etiology and risk factor profiles have been suggested. The subgroup of nonadvanced prostate cancers at diagnosis mainly encompasses less aggressive tumors, which progress slowly and might never become clinically relevant. Therefore, we considered advanced prostate cancer risk to be the most interesting outcome. MD adherence was not associated with advanced prostate cancer risk in our analyses. For nonadvanced prostate cancer risk, a statistically significant positive association was observed. The prostate cancer awareness, likelihood to seek care, and screening attendance may be higher among well-educated men with healthier lifestyles and higher MD adherence. Consequently, nonadvanced prostate tumors may more commonly be diagnosed in this part of the population. Additionally, we evaluated the association between MD adherence and bladder cancer risk combining male and female NLCS participants, and showed that there was no evidence of a relation, irrespective of the malignancy grade at diagnosis.

The association of MD adherence with overall cancer incidence was the focus of Chapter 8. Higher MD adherence was associated with a non-significantly reduced cancer risk in women, but not in men. In women, similar associations with MD adherence were observed for subgroups of cancers related vs. not related to tobacco smoking, obesity, and alcohol consumption. Even though differences across the subgroups seemed small and irrelevant in men as well, heterogeneity tests in men were significant for all subgroup comparisons 
made, possibly because of the high statistical power.

As was described above, our primary measure of MD adherence was aMEDr, which does not include the alcohol component. Largely similar results were obtained when MD adherence was assessed using the original aMED including the alcohol component. However, in most cases an equal or better model performance was observed for the MD score variant without alcohol.

In Chapter 9, the findings of this thesis were put into perspective by relating them to results of previously published studies and discussing methodological considerations. Moreover, implications for public health and recommendations for future research were addressed.

This thesis shows that higher MD adherence might be associated with a reduced risk of several cancer (sub)types in the Netherlands. Therefore, the MD could potentially be an interesting dietary approach in the prevention of cancer in the Dutch population. However, when looking at the totality of the evidence, no final conclusions regarding the cancerpreventive properties of the MD can be drawn at this time. Inverse associations in our analyses did not always reach statistical significance and the number of prospective cohort studies is still small for some cancer (sub)sites. In agreement with our findings, it has been suggested that associations with MD adherence might differ between the sexes and/or depend on the cancer subsite evaluated, but unfortunately, it is not common practice yet to report sex- and subtype-specific results. Accordingly, in future, well-designed cohort studies and randomized controlled trials are warranted, which might provide the additional evidence required to justify the promotion of the MD with the specific aim to prevent cancer. Until this is the case, policymakers in the Netherlands could consider using the MD as a framework to develop a healthy plant-based dietary strategy for the prevention of chronic diseases in general. 


\section{Samenvatting}

De ziekte kanker heeft vanwege haar hoge incidentie en sterfte een nadelige invloed op het leven van veel mensen. Naar schatting had ongeveer tien procent van de gediagnosticeerde kankers in Nederland in 2010 voorkomen kunnen worden door gezonde voeding. Het traditionele mediterraan dieet (MD) kan worden gedefinieerd als het voedingspatroon dat eind jaren '50, begin jaren '60 van de vorige eeuw kenmerkend was voor gebieden grenzend aan de Middellandse Zee waar olijven werden geteeld (Hoofdstuk 1). Het MD was een plantaardig dieet dat werd gekarakteriseerd door een hoge inname van groenten, fruit, peulvruchten, noten, volkoren graanproducten en olijfolie (een bron van enkelvoudig onverzadigde vetzuren). De inname van vlees en zuivelproducten was daarentegen laag. Alcohol werd geconsumeerd in matige hoeveelheden, met name gedurende de maaltijd. Er zijn meerdere gezondheidsvoordelen toegeschreven aan het MD, waaronder een verlaagde sterfte en een verlaagd risico op hart- en vaatziekten. Hoewel het mogelijk gunstige effect van het MD op het risico op kanker de afgelopen jaren in toenemende mate is onderzocht, is het huidige bewijs voor de meeste kankersoorten nog beperkt. Daarnaast waren de resultaten van onderzoeken niet altijd consistent en hadden veel studies een patiëntcontrole-opzet. Het patiëntcontrole-onderzoeksdesign is gevoelig voor bias, om welke reden cohortonderzoeken de voorkeur hebben. Tot slot verschilt het verband met het MD mogelijk tussen de geslachten en/of subtypen van kankersoorten. Dit is echter zelden in prospectieve studies onderzocht. Het doel van dit proefschrift was daarom om het verband tussen naleving van het MD en incidentie van zowel kanker in het algemeen als verscheidene kankersoorten (i.c. long-, borst-, slokdarm-, maag-, alvleesklier-, colorectaal-, prostaat- en blaaskanker) in Nederland te onderzoeken in een prospectieve setting. Hierbij hebben we ook aandacht besteed aan mogelijke verschillen in associaties tussen mannen en vrouwen en subtypen van de onderzochte kankersoorten. De mate van naleving van het MD werd vastgesteld met behulp van twee a priori gedefinieerde MD-scores, namelijk de 'alternate Mediterranean diet score' (aMED) en de 'modified Mediterranean diet score' (mMED). Alcoholconsumptie is een risicofactor voor meerdere vormen van kanker. Daarom hebben we gereduceerde varianten van aMED en mMED gecreëerd (respectievelijk aMEDr en mMEDr) waar alcoholconsumptie geen deel van uitmaakt. Vervolgens hebben we de 'performance' van modellen met gereduceerde (zonder alcohol) en originele (met alcohol) MD-scores vergeleken. Onder 'model performance' verstaan we de mate waarin een statistisch model de geobserveerde data verklaart. Voor ons onderzoek hebben we met name gebruik gemaakt van data van de 120.852 deelnemers van de Nederlandse Cohortstudie naar voeding en kanker (NLCS). Bij aanvang van deze studie in september 1986 waren de deelnemers tussen de 55 en 69 jaar oud. Om de statistische power te vergroten, hebben we de relatie tussen naleving van het MD en het risico op alvleesklierkanker onderzocht door resultaten van de NLCS en het Nederlandse cohort van de European Prospective Investigation into Cancer and Nutrition (EPIC-NL) samen te voegen. Het EPIC-NL cohort is gevormd tussen 1993 en 1997 en bestaat uit 40.011 mannen en vrouwen in de leeftijd van 20-70 jaar. NLCS-deelnemers zijn voor maximaal 20,3 jaar gevolgd voor het optreden van kanker. De mediane follow-up in EPIC-NL was 19,2 jaar.

De performance van de modellen was voor de meeste kankersoorten vergelijkbaar of beter wanneer de naleving van het MD werd vastgesteld met behulp van aMEDr dan wanneer dit was gebeurd met mMEDr. Daarom hebben we besloten dat aMEDr onze belangrijkste maat was in de bepaling van naleving van het MD. Tevens heeft de score variant zonder alcohol de voorkeur, omdat alcoholconsumptie een risicofactor is voor verscheidene kankersoorten. Hogere MD-scores (aMEDr) waren geassocieerd met een niet statistisch significant verlaagd 
risico op longkanker bij mannen en vrouwen (Hoofdstuk 2). De inverse verbanden leken het sterkst bij vrouwen en nooit-rokers. De sterkte van de associatie leek ook te variëren tussen de histologische longkanker-subtypen, met name bij mannen. Heterogeniteitstesten waren echter niet statistisch significant.

Een toename in aMEDr was ook geassocieerd met een niet statistisch significant verlaagde incidentie van postmenopauzale borstkanker bij vrouwelijke NLCS-deelnemers (Hoofdstuk 3). Stratificatie op basis van oestrogeenreceptor (ER) status liet zien dat het inverse verband sterker en enkel statistisch significant was voor het ER-negatieve subtype. We zagen een vergelijkbaar patroon voor de progesteronreceptor (PR) en gecombineerde ER/PR subtypen. Tot slot hebben we de resultaten van de NLCS gecombineerd met resultaten van eerder gepubliceerde cohortstudies in random-effects meta-analyses. De resultaten van deze metaanalyses kwamen overeen met de NLCS-bevindingen. MD-scores waren invers geassocieerd met het risico op postmenopauzale borstkanker, met name van het ER-negatieve subtype. Slokdarm- en maagkanker-subtypen, gedefinieerd op basis van respectievelijk histologie en anatomische locatie, verschillen mogelijk in etiologie. De relatie tussen naleving van het MD en het risico op slokdarm- en maagkanker-subtypen was het onderwerp van Hoofdstuk 4. Hogere MD-scores waren bij mannen geassocieerd met een significant verlaagd risico op plaveiselcelcarcinoom van de slokdarm (ESCC). aMEDr was echter niet geassocieerd met het risico op ESCC bij vrouwen of het risico op adenocarcinoom van de slokdarm (EAC) bij zowel mannen als vrouwen. Wat betreft de maagkanker-subtypen vonden we inverse verbanden tussen aMEDr en risico's op cardia en non-cardia adenocarcinoom van de maag (respectievelijk GCA en GNCA) bij beide geslachten. De gevonden verbanden waren echter alleen statistisch significant bij mannen.

Het verband tussen naleving van het MD en het risico op alvleesklierkanker werd in Hoofdstuk 5 onderzocht met behulp van data verzameld in de NLCS en EPIC-NL cohorten. aMEDr was niet statistisch significant geassocieerd met het risico op microscopisch bevestigde alvleesklierkanker (MCPC) in gepoolde en cohort-specifieke analyses, ongeacht geslacht. Er was sprake van mogelijke effectmodificatie door rookstatus. Er leek een zwak en niet significant invers verband aanwezig te zijn bij nooit-rokers, terwijl hiervoor geen aanwijzingen waren bij onderzoeksdeelnemers die wel ooit hadden gerookt. De conclusie was vergelijkbaar wanneer ook niet-microscopisch bevestigde gevallen van alvleesklierkanker in de analyses werden meegenomen.

aMEDr was eveneens niet statistisch significant geassocieerd met het risico op colorectaalkanker (Hoofdstuk 6). Dit was het geval voor alle onderzochte anatomische subtypen (i.e. colon, proximale colon, distale colon en rectum) bij zowel mannen als vrouwen.

In Hoofdstuk 7 hebben we associaties tussen naleving van het MD en het risico op prostaaten blaaskanker onderzocht. Associaties met prostaatkankerrisico zijn apart geschat voor 'advanced' (oftewel van een gevorderd stadium) en 'nonadvanced' tumoren op het moment van diagnose. Deze tumoren verschillen mogelijk in etiologie en risicofactoren. De subgroep van 'nonadvanced' prostaatkankers bestaat met name uit minder agressieve tumoren die mogelijk nooit een klinisch relevant stadium zullen bereiken. Daarom beschouwen we het risico op 'advanced' prostaatkanker als de belangrijkste uitkomstmaat. aMEDr was in onze analyses niet geassocieerd met het risico op 'advanced' prostaatkanker. Een statistisch significant positief verband werd echter gevonden voor 'nonadvanced' prostaatkanker. Hierbij moet worden opgemerkt dat mannen met een hoger opleidingsniveau en een gezondere leefstijl zich mogelijk meer bewust zijn van prostaatkanker en wellicht ook eerder gebruik zullen maken van de zorg en deel zullen nemen aan screeningsprogramma's. Daarom is het aannemelijk dat 'nonadvanced' prostaattumoren in dit deel van de populatie 
vaker gediagnosticeerd worden. Er was geen indicatie voor een associatie tussen aMEDr en het risico op blaaskanker. Onze bevindingen waren vergelijkbaar voor blaaskanker in het algemeen, invasieve blaaskanker en niet-invasieve blaaskanker. De effectschattingen voor blaaskanker werden gebaseerd op zowel mannen als vrouwen.

De relatie tussen naleving van het MD en het risico op kanker in het algemeen was de focus van Hoofdstuk 8. Hogere MD-scores waren geassocieerd met een niet significant verlaagd risico op kanker bij vrouwen. Er was echter geen sprake van een relatie bij mannen. Tevens hebben we kankersoorten ingedeeld in subgroepen op basis van de relatie met roken, obesitas en alcoholconsumptie. Associaties met aMEDr waren bij vrouwen voor de verschillende subgroepen vergelijkbaar. Ofschoon ook bij mannen de verschillen tussen de subgroepen klein en irrelevant leken, waren heterogeniteitstesten bij mannen significant voor alle vergelijkingen. Deze observatie kan wellicht worden verklaard door de hoge statistische power. Zoals eerder beschreven was in dit proefschrift aMEDr (zonder alcohol) de primaire maat voor vaststelling van naleving van het MD. Grotendeels vergelijkbare resultaten werden verkregen wanneer we de originele aMED (inclusief alcohol) gebruikten. De model performance was in de meeste gevallen echter vergelijkbaar of beter voor de MD-score variant zonder alcohol.

In Hoofdstuk 9 hebben we geprobeerd om de bevindingen van dit proefschrift in perspectief te plaatsen door deze te relateren aan resultaten van eerdere studies en door beschouwing van enkele methodologische aspecten. Daarnaast worden hier implicaties voor de volksgezondheid en aanbevelingen voor vervolgonderzoek behandeld.

Op basis van dit proefschrift kunnen we concluderen dat naleving van het MD mogelijk verband houdt met een verlaagd risico op verschillende kanker(sub)typen in Nederland. Het MD zou daarom wellicht een interessante voedingsstrategie zijn voor de preventie van kanker in de Nederlandse samenleving. Het huidige bewijs volstaat echter nog niet voor de formulering van definitieve conclusies omtrent het mogelijk risicoverlagend effect van het MD op kanker. De inverse verbanden die we in dit proefschrift vonden, waren niet altijd statistisch significant en het aantal prospectieve cohortstudies is voor sommige kanker(sub)typen nog steeds beperkt. Zowel onze bevindingen als de literatuur wekken de indruk dat associaties met naleving van het MD mogelijk verschillen tussen de geslachten en/of afhankelijk zijn van het kankersubtype dat wordt onderzocht. Onderzoeksresultaten worden in studies helaas nog lang niet altijd gespecificeerd naar geslacht en subtype. Toekomstige goed uitgevoerde cohort- en interventiestudies kunnen wellicht het bewijs leveren dat noodzakelijk is om de promotie van het MD in het licht van kankerpreventie te rechtvaardigen. Totdat dit daadwerkelijk het geval is kunnen beleidsmakers in Nederland overwegen om het MD als uitgangspunt te nemen voor de ontwikkeling van een gezond plantaardig voedingspatroon gericht op de preventie van chronische ziekten in het algemeen. 


\section{Valorization}

The term "valorization" refers to the process of creating societal or economic value from scientific knowledge. In addition to education and research, valorization by law constitutes the third core task of Dutch universities. In this section, we will discuss how our key findings with regard to the potential cancer-protective effect of the Mediterranean diet (MD) in a non-Mediterranean population can be of significance to society apart from their scientific value through publication in impact journals.

Before elaborating on the valorization potential of our findings, we will first briefly introduce the concept of the MD and its key components. In this thesis, the traditional MD was defined as "the dietary pattern typical of the Mediterranean regions traditionally known for olive cultivation in the late 1950 s and the early 1960s". Consumption of plant foods (e.g., vegetables, fruits, legumes, nuts, and whole grains) was abundant in this dietary pattern, whereas the intake of animal foods (e.g., meat and dairy) was limited. Other characteristics of the traditional MD were the high ratio of monounsaturated to saturated fatty acids resulting from the generous consumption of olive oil and a moderate consumption of alcohol during meals $[1,2]$.

A considerable part of cancer cases is presumably preventable with healthy dietary habits. For the Dutch population, it has been estimated that approximately $10 \%$ of cancer diagnoses in 2010 could be ascribed to a less than optimal diet [3]. Society could benefit from nutritional research through the translation of results into dietary guidelines. For example, the World Cancer Research Fund/American Institute for Cancer Research (WCRF/ AICR) has formulated nine recommendations for cancer prevention in their Third Expert Report in 2018 [4] after systematic review of the scientific literature focusing on the relation of diet, nutrition, and physical activity to cancer. According to the Expert Panel, the evidence with respect to a "Mediterranean type" dietary pattern was still inadequate to allow a meaningful recommendation. The findings of this thesis may be included in possible future Expert Reports of the WCRF/AICR and in this way contribute to the formulation of international lifestyle guidelines for cancer prevention.

Regarding the potential of the MD as a dietary strategy specifically aimed at cancer prevention, our results suggested that in the Netherlands, MD adherence may be associated with reduced risks of cancers of the lung, female breast (postmenopausal), esophagus (squamous cell carcinoma in men), and stomach. The prognosis of most of these cancer sites is relatively poor, stressing the importance of preventive strategies. However, as already discussed in Chapter 9 of this thesis, the currently available evidence does probably not suffice to recommend Dutch policymakers to promote the MD specifically for cancer prevention at this time.

In addition to its potentially favorable effect on cancer risk, MD adherence may be associated with various other health benefits, including reduced mortality and lower risks of several chronic diseases. This enables the formulation of a clear, consistent (with respect to disease risk) message to society and is likely to enhance the usability of the MD as a dietary strategy to improve the health status of the population. Furthermore, the MD is generally considered a palatable dietary pattern with a relatively low impact on the environment. Policymakers in the Netherlands could possibly use the MD as a framework to develop a healthy plant-based dietary pattern with the purpose of preventing chronic disease in general (see Chapter 9 for a more elaborate discussion).

The successful implementation of a healthy plant-oriented dietary pattern in the Dutch 
population most likely requires an integrative approach involving organized efforts of the government and society as a whole [4]. Policies are warranted that enable and encourage the adoption of the promoted dietary pattern by the community. In order to achieve these aims, such policy actions should influence the three domains of food environment, food system, and behavior change communication [4]. The behavior change domain encompasses educating people about health effects of food and nutrition by raising public awareness [4]. In this respect, (inter)national media attention has been paid to the health benefits of the MD in recent years. For example, our scientific article concerning MD adherence and postmenopausal breast cancer risk has been highlighted on (inter)national news websites. In addition to "unregulated" media attention, which is often concentrated on recent research papers showing positive health effects of MD adherence, Dutch authorities could play a role in promoting and disseminating a MD-derived plant-oriented dietary pattern in a more organized manner. In this instance, the Health Council of the Netherlands ("Gezondheidsraad") and the Netherlands Nutrition Centre ("Voedingscentrum") could get involved. Over the past decades, the Health Council of the Netherlands has issued several recommendations for a healthy dietary pattern targeted at the general Dutch population, with the most recent update being published in 2015 [5]. The Dutch dietary guidelines 2015 [5] were formulated by taking the latest scientific evidence concerning chronic disease risk into consideration and integrate information regarding nutrients, foods, and dietary patterns. According to the Dutch dietary guidelines 2015, recommended dietary patterns (including the traditional MD) characterized by a higher consumption of plant foods and a lower consumption of foods from animal origin, positively affect health [5]. The Netherlands Nutrition Centre is a leading authority that is committed to advising the public and health care professionals about healthy and more sustainable dietary habits and aims to encourage people to change their current eating habits accordingly [6]. The Netherlands Nutrition Centre has created the "Wheel of Five" ("Schijf van Vijf") [5-7]. The "Wheel of Five" 2016 (Figure 1) is a practical translation of the Dutch dietary guidelines 2015 for use in nutritional counseling, which has been complemented with specific recommendations in order to make sure that people meet their energy and nutrient requirements. Activities of the Netherlands Nutrition Centre promoting the dissemination of the "Wheel of Five" by health care professionals include educating this group about the "Wheel of Five" and its components in general, as well as its development and expected health benefits. Furthermore, information, materials, and tools (e.g., leaflets, posters, and explanatory videos) are provided to support the use of the "Wheel of Five" in clinical practice. For the general population, the website of the Netherlands Nutrition Centre contains a wealth of information and tools to help and encourage individuals to make healthier food choices. Along with a description of the "Wheel of Five", the website features advice, recipes, daily meal plans, and e-tools (e.g., "Schijf van Vijf voor jou" and "Mijn eet-update") to inspire people [6, 7].

In addition to the actions suggested above, informative presentations concerning the potential health benefits of the MD could be given at conferences for health care professionals, who could in turn transfer this knowledge to patients via nutritional counseling.

When confirmed, the findings of this thesis could contribute to a decrease in morbidity and mortality due to cancer (and possibly other chronic diseases) in the Netherlands through the formulation of dietary guidelines and an increased public awareness of the impact of diet and other lifestyle factors on people's health. Interestingly, increasing adherence to the MD seemed to be especially associated with reduced risks of cancers with a relatively poor prognosis. In addition to a reduced social burden, the prevention of cancer may have economic benefits, such as decreased health care costs and a reduced loss of productivity 


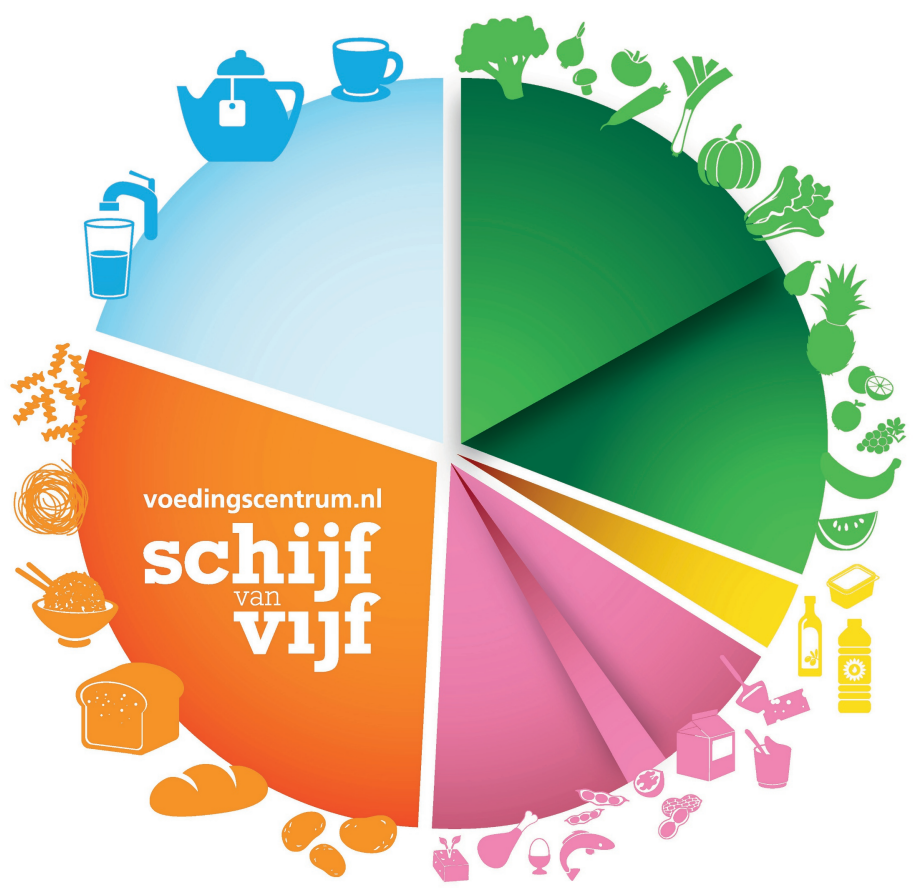

Figure 1 The "Wheel of Five" ("Schijf van Vijf") 2016

at the workplace. Finally, adoption of a Mediterranean(-like) dietary pattern by the Dutch population is likely to have advantageous effects for the environment as well [8-13]. A study by Van Dooren et al. [11] compared greenhouse gas emissions and land use associated with six dietary patterns, including the average Dutch diet of 1998 and the MD, in female adults. Greenhouse gas emissions and land use for the MD were estimated to be clearly lower than for the average Dutch diet [11], which underscores the benefits of adopting a Mediterranean(-like) dietary pattern. 
Addendum

\section{References}

1. Willett WC, Sacks F, Trichopoulou A, Drescher G, Ferro-Luzzi A, Helsing E, et al. Mediterranean diet pyramid: a cultural model for healthy eating. Am J Clin Nutr. 1995;61(6 Suppl):1402S-6S.

2. Trichopoulou A, Lagiou P. Healthy traditional Mediterranean diet: an expression of culture, history, and lifestyle. Nutr Rev. 1997;55(11 Pt 1):383-9.

3. Lanting $\mathrm{Cl}$, de Vroome EM, Elias SG, van den Brandt PA, van Leeuwen FE, Kampman E, et al. [Contribution of lifestyle factors to cancer: secondary analysis of Dutch data over 2010 and a projection for 2020]. Ned Tijdschr Geneeskd. 2014;159:A8085 (in Dutch).

4. World Cancer Research Fund / American Institute for Cancer Research. Diet, nutrition, physical activity and cancer: a global perspective. Continuous update project expert report 2018. 2018. Available from: https://www.wcrf.org/dietandcancer.

5. Health Council of the Netherlands. Richtlijn goede voeding 2015. The Hague, the Netherlands: Health Council of the Netherlands; 2015; publication number: 2015/24 (in Dutch).

6. Netherlands Nutrition Centre [Website]. The Hague, the Netherlands: Netherlands Nutrition Centre; 2020 [cited 2020 January]. Available from: https://voedingscentrum.nl/nl.aspx.

7. Brink L, Postma-Smeets A, Stafleu A, Wolvers D. Richtlijnen Schijf van Vijf, 6e druk. The Hague, the Netherlands: Netherlands Nutrition Centre; 2019 (in Dutch).

8. Tukker A, Goldbohm RA, de Koning A, Verheijden M, Kleijn R, Wolf O, et al. Environmental impacts of changes to healthier diets in Europe. Ecol Econ. 2011;70(10):1776-88.

9. Heller MC, Keoleian GA, Willett WC. Toward a life cycle-based, diet-level framework for food environmental impact and nutritional quality assessment: a critical review. Environ Sci Technol. 2013;47(22):12632-47.

10. Vanham D, Mekonnen MM, Hoekstra AY. The water footprint of the EU for different diets. Ecol Indic. 2013;32:1-8.

11. van Dooren C, Marinussen M, Blonk H, Aiking H, Vellinga P. Exploring dietary guidelines based on ecological and nutritional values: A comparison of six dietary patterns. Food Policy. 2014;44:36-46.

12. Tilman D, Clark M. Global diets link environmental sustainability and human health. Nature. 2014;515(7528):518-22.

13. Dernini S, Berry EM, Serra-Majem L, La Vecchia C, Capone R, Medina FX, et al. Med Diet 4.0: the Mediterranean diet with four sustainable benefits. Public Health Nutr. 2017;20(7):1322-30. 
Valorization 


\section{Dankwoord}

Tijdens de afgelopen vier jaar als promovenda heb ik me ontwikkeld als wetenschapper en ben ik gegroeid als persoon. Hierbij heb ik de hulp gehad van mijn promotoren, collega's, vrienden en familie. Zonder hun bijdragen, onvoorwaardelijke steun, vertrouwen, feedback en motiverende woorden was het me nooit gelukt om tot zo'n mooi resultaat te komen. Bedankt allemaal!

Allereerst wil ik mijn promotieteam, bestaande uit prof. dr. ir. Piet A. van den Brandt en prof. dr. Petra H.M. Peeters, in het bijzonder bedanken.

Piet, bedankt voor het vertrouwen! Jij zag de potentie in mij toen ik vier jaar geleden bij je solliciteerde en hebt mij toen de kans geboden om me via dit promotietraject verder te ontwikkelen als epidemiologisch onderzoeker. Ik heb veel geleerd van je deskundigheid, kritische blik en constructieve feedback. Je draaide tijdens onze gesprekken nooit om de feiten heen. Alhoewel ik er op het moment zelf soms anders over dacht, waardeer ik deze directheid zeer. In de loop van de jaren ben je er vast achter gekomen dat ik zo nu en dan best eigenwijs kan zijn. Tijdens onze gesprekken toonde je ook regelmatig interesse in mij als persoon en liet je je 'vaderlijke' kant zien. Daarnaast luisterde ik graag naar je ervaringen als (jonge) onderzoeker, waar je menigmaal met enthousiasme over vertelde. Ondanks dat je het vaak druk had, voelde het alsof je deur altijd voor mij openstond en kon je, als het nodig was, steevast een plaatsje voor mij vrijmaken in je agenda.

Petra, jij was bij mijn promotieonderzoek betrokken als expert op het gebied van de EPICNL data. Je frisse kijk op de resultaten en artikelen was altijd zeer zinvol. Daarnaast wil ik je bedanken voor je positieve instelling, motiverende woorden en betrokkenheid. Ik heb je leren kennen als een open, aardig en warm persoon.

Daarnaast wil ik alle NLCS- en EPIC-NL deelnemers hartelijk danken. Dit promotieonderzoek was niet mogelijk geweest zonder hen! Tevens dank aan het NLCS-team voor de ondersteuning, waardevolle adviezen en methodologische discussies. Het onderzoeksteam van EPIC-NL wil ik ook graag bedanken voor de prettige samenwerking en het delen van de onderzoeksgegevens.

De leden van de beoordelingscommissie, prof. dr. Theo M.C.M. de Kok, prof. dr. ir. Edith J.M. Feskens, prof. dr. Ad A.M. Masclee, prof. dr. Anne M. May en prof. dr. Hein de Vries, wil ik bedanken voor de tijd en moeite die ze hebben gestoken in het kritisch lezen en het beoordelen van dit proefschrift.

Collega's Epidemiologie, de afgelopen jaren heb ik met veel plezier op onze afdeling gewerkt. Bedankt voor de prettige werksfeer, de gezellige gesprekken in het keukentje (soms al in de vroege ochtend) en onze legendarische (en nimmer saaie) dagjes-uit.

Bij Yvonne, Mariëlle en Petra (de dames van het secretariaat), Jolanda en Conny, kon ik altijd terecht met mijn vragen over van alles en nog wat. Bedankt voor jullie hulp en ondersteuning. Yvonne en Mariëlle, jullie wil ik in het bijzonder bedanken voor de energie en tijd die jullie hebben gestoken in alle administratieve zaken rondom dit proefschrift en mijn verdediging. Harry en Jos, de ICT-experts van de afdeling, jullie stonden altijd paraat met een snelle en goede oplossing als ik weer eens problemen had met mijn computer. Jullie hulp was onmisbaar! 
(Oud-)aio's, ik kijk met veel plezier terug op onze lunches, borrels en etentjes, die hebben gezorgd voor de nodige gezelligheid en afwisseling. Ik ben blij dat ik jullie heb leren kennen! Lloyd, het was fijn dat ik de afgelopen jaren iemand had die in dezelfde fase van zijn PhDtraject zat. Af en toe heb ik er ook handig gebruik van gemaakt dat jij met alles net een maandje voorliep. Op de kamer die je met Jeroen en Lisette deelde was er altijd ruimte om te praten over serieuze (en iets minder serieuze) zaken. Van inhoudelijke discussies tot persoonlijke kwesties, weekendplannen en 'foute' woordgrappen, alles kwam aan bod. Onze 'kamelenrace' en 'manuscript rejection ranking' mogen hier natuurlijk ook niet ontbreken! Daarnaast gingen we tijdens de lunchpauze regelmatig, regen of geen regen, een rondje lopen. Door de jaren heen is ons wandelgroepje steeds groter geworden en door alle 'training' (en de inspanningen van Jeroen en Eline) ging het looptempo flink omhoog en hoefden we steeds minder werktijd te missen.

Karlijn, jij stond altijd voor me klaar. Dank je wel voor de gezellige avondmaaltijden samen en onze fijne gesprekken.

Annaleen en Rachel, met jullie heb ik voor langere perioden een kamer, en het nodige lief en leed, gedeeld. Jullie waren altijd bereid om me advies te geven, boden me een luisterend oor en zorgden soms voor de noodzakelijke afleiding.

Tot slot wil ik mijn paranimfen, Karlijn en Lisette, hier nogmaals noemen. Bedankt dat jullie bij mijn verdediging achter me willen staan!

Bas en Gwen, inmiddels zijn we al ruim 10 jaar bevriend. Samen hebben we veel meegemaakt sinds onze kennismaking op de middelbare school. Gwen, je woont nu alweer een tijdje in Londen, maar gelukkig kom je nog regelmatig naar Nederland en je weet dan ook altijd tijd voor Bas en mij vrij te maken. Je bent mijn redder in nood bij taalkundige vragen en hebt op dat gebied ook een belangrijke bijdrage geleverd aan dit proefschrift. Bas, als ik weer ergens over zit te stressen ben je altijd bereid om je nuchtere en relativerende blik over de kwestie te laten schijnen. Ik wil jullie bedanken voor alle gezelligheid en steun. Hopelijk blijft onze vriendschap voor altijd bestaan.

Jeanine, wie had tijdens onze studie gedacht dat ik hier nu zou staan. De afgelopen jaren zijn voorbijgevlogen. Hoewel we elkaar maar enkele keren per jaar zien, is het altijd alsof we elkaar gisteren nog hebben gesproken. Bedankt voor alle gezellige uitjes, onze prettige gesprekken en je goede adviezen. Door jou kom ik op plaatsen in Nederland waar ik nooit eerder geweest ben. Ik kan altijd mijn hart bij je luchten. Ik kijk ernaar uit om samen in de toekomst ook de rest van Nederland (en misschien wel Europa) te gaan verkennen.

Lieve ooms, tantes, (achter-)nichten en neven, bedankt voor jullie warmte, mentale support en belangstelling voor mijn onderzoek. Nu kunnen jullie eindelijk zien waar ik de afgelopen vier jaar mee bezig ben geweest.

Kitty, met je deskundigheid op het gebied van de Nederlandse (en Engelse) taal en je taalkundige bijdrage aan deze thesis verdien jij een speciale vermelding in dit dankwoord.

Lieve opa's, oma's en tante Lucie, jammer genoeg kunnen jullie deze mijlpaal niet meer meemaken. Wat zouden jullie trots zijn geweest!

Lieve papa, mama en Robbert, bedankt voor alles! Hoewel de afgelopen jaren zeker niet gemakkelijk waren, stonden jullie altijd voor me klaar en hebben jullie mij geholpen waar mogelijk. Zonder jullie onvoorwaardelijke liefde, steun en vertrouwen, en de soms noodzakelijke duwtjes in de rug, zou dit proefschrift niet hebben bestaan. Ik houd ontzettend veel van jullie en ben trots en dankbaar dat ik jullie dochter/grote zus mag zijn! 
Dankwoord 


\section{Curriculum Vitae}

Maya Schulpen was born on the $10^{\text {th }}$ of February 1992 in Eindhoven, the Netherlands. After graduating secondary school with honors at Pleincollege Bisschop Bekkers in Eindhoven in 2010, Maya studied Biomedical Sciences at Radboud University in Nijmegen. During her master's education, she specialized in the fields of human pathobiology and epidemiology. As an intern, she investigated the effects of amyloid- $\beta$ and small heat shock proteins on microglial inflammation in Alzheimer's disease at the Departments of Neurology and Laboratory Medicine at the Radboud University Medical Center. Her second master's internship was focused on the effects of occupational exposure to endocrine disruptors and

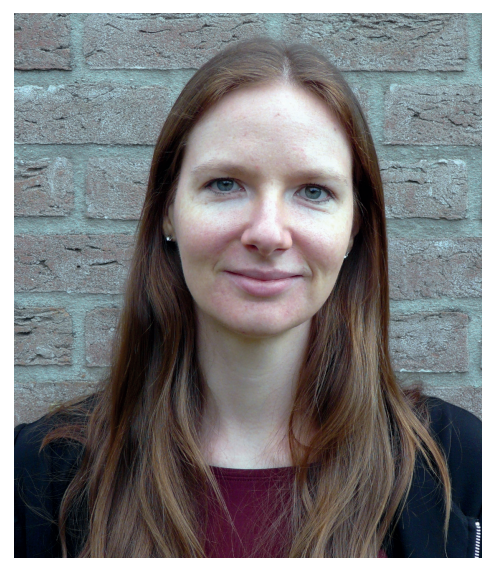
work-related stress on birth weight and duration of pregnancy, and was performed at the Department for Health Evidence at the Radboud University Medical Center. Maya obtained her bachelor's and master's degrees in Biomedical Sciences (both Cum Laude) in 2013 and 2016, respectively. Consecutively, she started as a PhD student at the Department of Epidemiology at Maastricht University (GROW- School for Oncology and Developmental Biology) under the supervision of professor Piet A. van den Brandt and professor Petra H. M. Peeters. Her PhD research was aimed at investigating the association between adherence to a Mediterranean diet and cancer risk in the Netherlands using data from the Netherlands Cohort Study (NLCS) and the Dutch cohort of the European Prospective Investigation into Cancer and Nutrition (EPIC-NL). The scientific results, which are presented in this thesis, have been reported in peer-reviewed publications and have been presented at (inter)national congresses. Maya is registered as Epidemiologist $A$ at the Dutch Society for Epidemiology (VvE). 


\section{List of publications}

\section{Published papers}

- van den Brandt PA, Schulpen M. Mediterranean diet adherence and risk of postmenopausal breast cancer: results of a cohort study and meta-analysis. Int J Cancer. 2017; 140(10): 2220-2231. doi: 10.1002/ijc.30654.

- $\quad$ van der Doelen RHA, Robroch B, Arnoldussen IA, Schulpen M, Homberg JR, Kozicz T. Serotonin and urocortin 1 in the dorsal raphe and Edinger-Westphal nuclei after early life stress in serotonin transporter knockout rats. Neuroscience. 2017; 340: 345-358. doi: 10.1016/j.neuroscience.2016.10.072.

- Winkler LA, Frølich JS, Schulpen M, Støving RK. Body composition and menstrual status in adults with a history of anorexia nervosa - at what fat percentage is the menstrual cycle restored? Int J Eat Disord. 2017; 50(4): 370-377. doi: 10.1002/ eat.22600.

- Schulpen M, van den Brandt PA. Adherence to the Mediterranean diet and risk of lung cancer in the Netherlands Cohort Study. Br J Nutr. 2018; 119(6): 674-684. doi: 10.1017/S0007114517003737.

- Schulpen M, Peeters PH, van den Brandt PA. Mediterranean diet adherence and risk of pancreatic cancer: A pooled analysis of two Dutch cohorts. Int J Cancer. 2019; 144(7): 1550-1560. doi: 10.1002/ijc.31872.

- Schulpen M, Peeters PH, van den Brandt PA. Mediterranean diet adherence and risk of esophageal and gastric cancer subtypes in the Netherlands Cohort Study. Gastric Cancer. 2019; 22(4): 663-674. doi: 10.1007/s10120-019-00927-x.

- Schulpen M, van den Brandt PA. Adherence to the Mediterranean Diet and Risks of Prostate and Bladder Cancer in the Netherlands Cohort Study. Cancer Epidemiol Biomarkers Prev. 2019; 28(9): 1480-1488. doi: 10.1158/1055-9965.EPI-19-0224.

- Schulpen M, van den Brandt PA. Mediterranean diet adherence and risk of colorectal cancer: the prospective Netherlands Cohort Study. Eur J Epidemiol. 2020; 35(1): 25-35. doi: 10.1007/s10654-019-00549-8.

\section{Submitted papers}

- Schulpen M, van den Brandt PA. Adherence to the Mediterranean diet and overall cancer incidence: the Netherlands Cohort Study. 
